\title{
Catalytic Enantioselective Halocyclizations to Access Benzoxazepinones and Benzoxazecinones
}

\author{
Xiaojian Jiang, ${ }^{, \dagger} \mathrm{Xi} \mathrm{Xu},{ }^{\dagger}$ Wei Xu,${ }^{\dagger}$ Pei Yu,${ }^{\dagger}$ and Ying-Yeung Yeung $*$, \\ IInternational Cooperative Laboratory of Traditional Chinese Medicine Modernization and Innovative Drug \\ Development of Chinese Ministry of Education (MOE), College of Pharmacy, Jinan University, Guangzhou 510632, \\ China \\ * Department of Chemistry and State Key Laboratory of Synthetic Chemistry, The Chinese University of Hong Kong, \\ Shatin, NT, Hong Kong (China) \\ E-mail: chemjxj2015@jnu.edu.cn; yyyeung@cuhk.edu.hk
}




\section{Table of Contents}

\section{GENERAL METHODS}

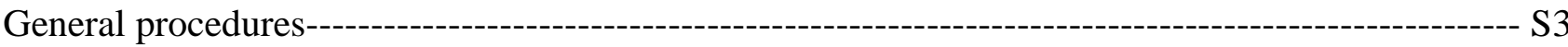

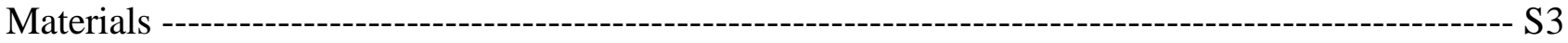

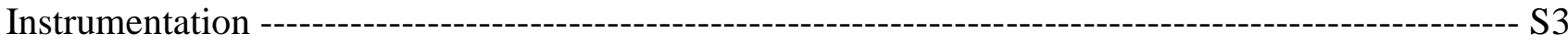

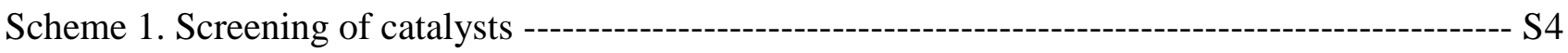

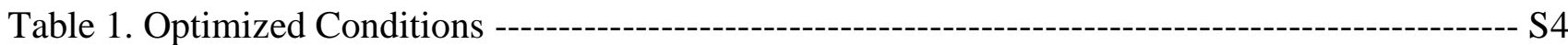

Figure 1. ${ }^{1} \mathrm{H}-\mathrm{NMR}$ analysis of NBS and (DHQ) ${ }_{2} \mathrm{PHAL}$------------------------------------------------ S5

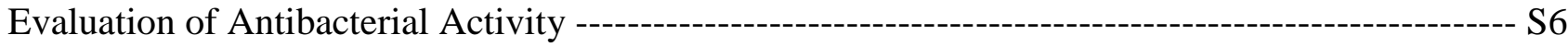

SYNTHESIS OF CATALYSTS

Synthetic Procedure of $(\mathrm{Cin})_{2} \mathrm{PHAL}$ and (ECin $)_{2} \mathrm{PHAL}$

\section{SYNTHESIS OF ALKENOIC ACID SUBSTRATES AND ASYMMETRIC}

\section{HALOLACTONIZATION REACTIONS}

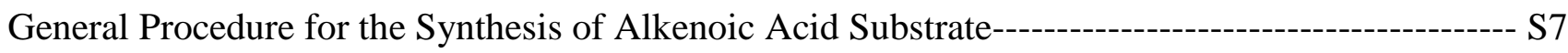

Representative Procedure for the Synthesis of Alkenoic Acid Substrate 1a---------------------------- S8

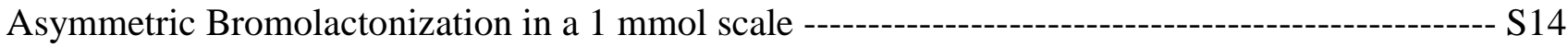

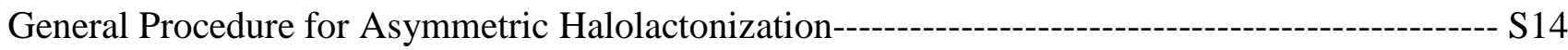

Asymmetric Chlorolactonization of 7---_-_-

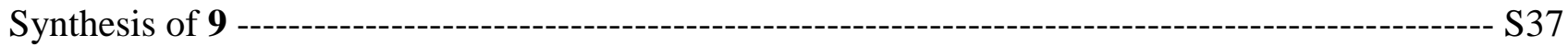

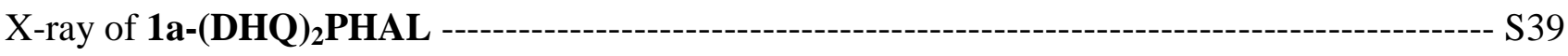

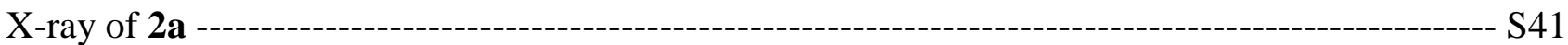

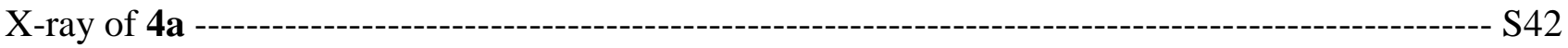

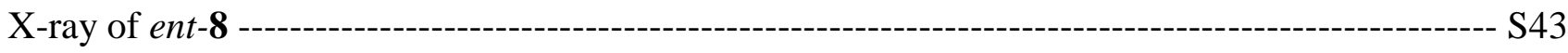

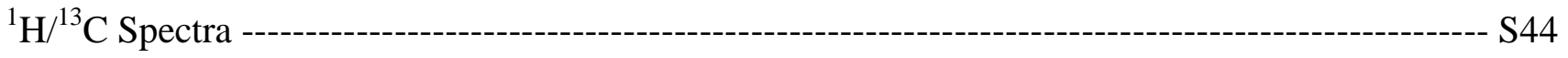




\section{GENERAL METHODS}

\section{General Procedures}

All reactions were generally performed open air or in dried glassware under an atmosphere of dry $\mathrm{N}_{2}$. Reaction mixtures were stirred magnetically unless otherwise indicated and monitored by thin layer chromatography (TLC) on Merck precoated glass-backed silica gel 60 F-254 0.25 mm plates with visualization by fluorescence quenching at $254 \mathrm{~nm}$. TLC plates were stained using potassium permanganate. Chromatography purification of products (flash column chromatography) was performed on silica gel 60 (70-230 mesh, Merck) using a forced flow of eluent at 0.3-0.5 bar. Concentration of reaction product solutions and chromatography fractions under reduced pressure was performed by rotary evaporation at $35-45^{\circ} \mathrm{C}$ at the appropriate pressure and then at rt, ca. 10 $\mathrm{mmHg}$ (vacuum pump) unless otherwise indicated.

\section{Materials}

All chemicals, including dry solvents were purchased from Aldrich, Fluka, Acros, TCI, Merck, Strem, or Alfa Aesar and used as such unless stated otherwise. Yields given refer to chromatographically purified compounds unless otherwise demonstrated.

\section{Instrumentation}

Melting points were determined on a Sinoinstructment melting point apparatus. ${ }^{1} \mathrm{H}$ NMR spectra were recorded on Bruker $300 \mathrm{MHz}$ or Bruker $400 \mathrm{MHz}$ spectrometer. ${ }^{13} \mathrm{C}$ NMR spectra were recorded on Bruker $75 \mathrm{MHz}$ or Bruker $100 \mathrm{MHz}$ spectrometer. ${ }^{13} \mathrm{C}$ NMR chemical shifts are expressed in parts per million ( $\delta$ ) downfield from tetramethylsilane (with the central peak of $\mathrm{CHCl}_{3}$ at $77.16 \mathrm{ppm}$ used as standard). ${ }^{1} \mathrm{H}$ NMR chemical shifts are expressed in parts per million $(\delta)$ downfield from tetramethylsilane (with the peak of $\mathrm{CHCl}_{3}$ at $7.26 \mathrm{ppm}$ used as standard; with the peak of benzene at 7. $36 \mathrm{ppm}$ used as standard). All ${ }^{13} \mathrm{C}$ spectra were measured with complete proton decoupling. NMR coupling constants $(\mathrm{J})$ are reported in Hertz $(\mathrm{Hz})$, and splitting patterns are indicated as follows: br, broad; s, singlet; d, doublet; dd, doublet of doublet; ddd, doublet of doublet of doublet; $\mathrm{dt}$, doublet of triplet; $\mathrm{t}$, triplet; q, quartet; $\mathrm{m}$, multiplet. High resolution mass spectrometric measurements (HRMS) were performed by the AB SCIEX, Triple TOF 5600+. Enantiomeric

excesses were determined by HPLC analysis on Shimadzu HPLC units, including the following instruments: pump, LC-16; detector, SPD-16; column, Daicel Chiralpak IC, AD-H, OD-H, OJ-H or IC. 
Scheme 1. Screening of catalysts ${ }^{[a]}$

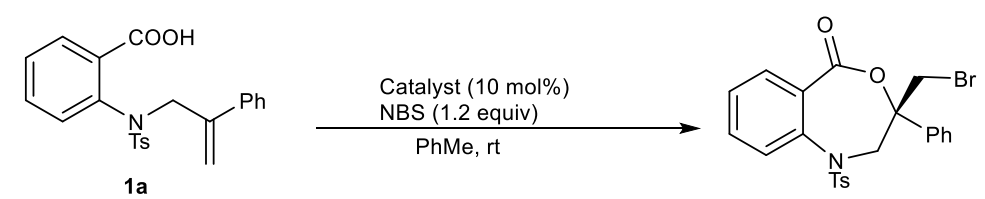

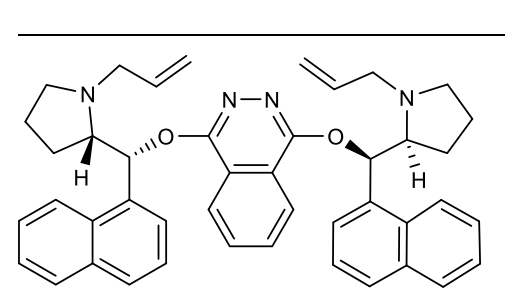

C1, $23 \%$ ee

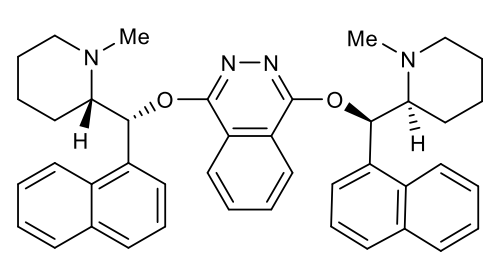

C2, $0 \%$ ee

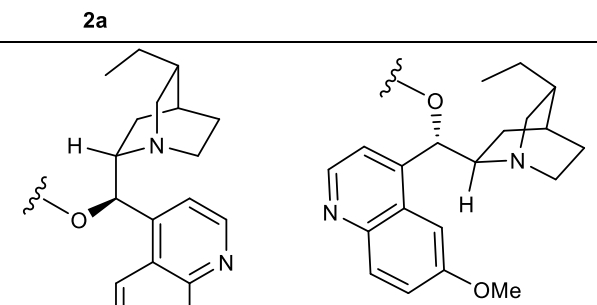

DHQD<smiles>C=CCN1CCC[C@H]1[C@@H]([14CH3])OC(=S)Nc1ccccc1</smiles>

C3, $0 \%$ ee

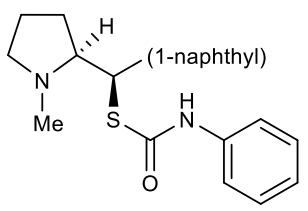

C4, $0 \%$ ee<smiles>Oc1nnc(O)c2ccccc12</smiles>

$(\mathrm{DHQ})_{2} \mathrm{PHAL}, 99 \%$ ee<smiles>C=CC1CC2CCN1C2[C@H](NC(=O)Nc1ccccc1)c1ccnc2ccc(OC)cc12</smiles><smiles>C=CC1CC2CCN1C2[C@H](NC(=O)Nc1ccccc1)c1ccnc2ccc(OC(C)(C)C)cc12</smiles><smiles>C=CC1CC2CCN1CC2[C@H](OC(=S)Nc1ccccc1)c1ccccc1O</smiles>

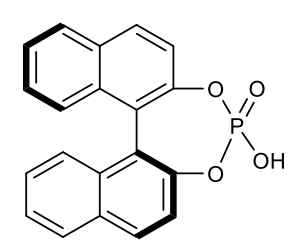

C2, $11 \%$ ee

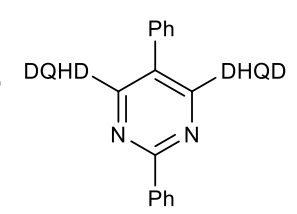

(DHQD) ${ }_{2} \mathrm{Pyr}, 67 \%$ ee

${ }_{2} \mathrm{PHAL}$,

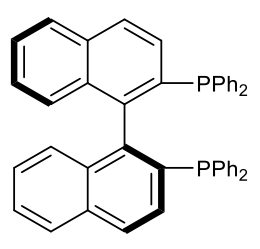

C2, $21 \%$ ee

[a] Reactions were carried out with substrate $1 \mathrm{a}(0.1 \mathrm{mmol})$, catalyst $(0.01 \mathrm{mmol})$, and halogen source $(0.12 \mathrm{mmol})$ in $\mathrm{PhMe}(4 \mathrm{~mL})$ at $25{ }^{\circ} \mathrm{C}$. The yield was isolated yield and the ee was determined by chiral HPLC.

\section{Table 1. Optimized Conditions ${ }^{[\mathrm{a}]}$}
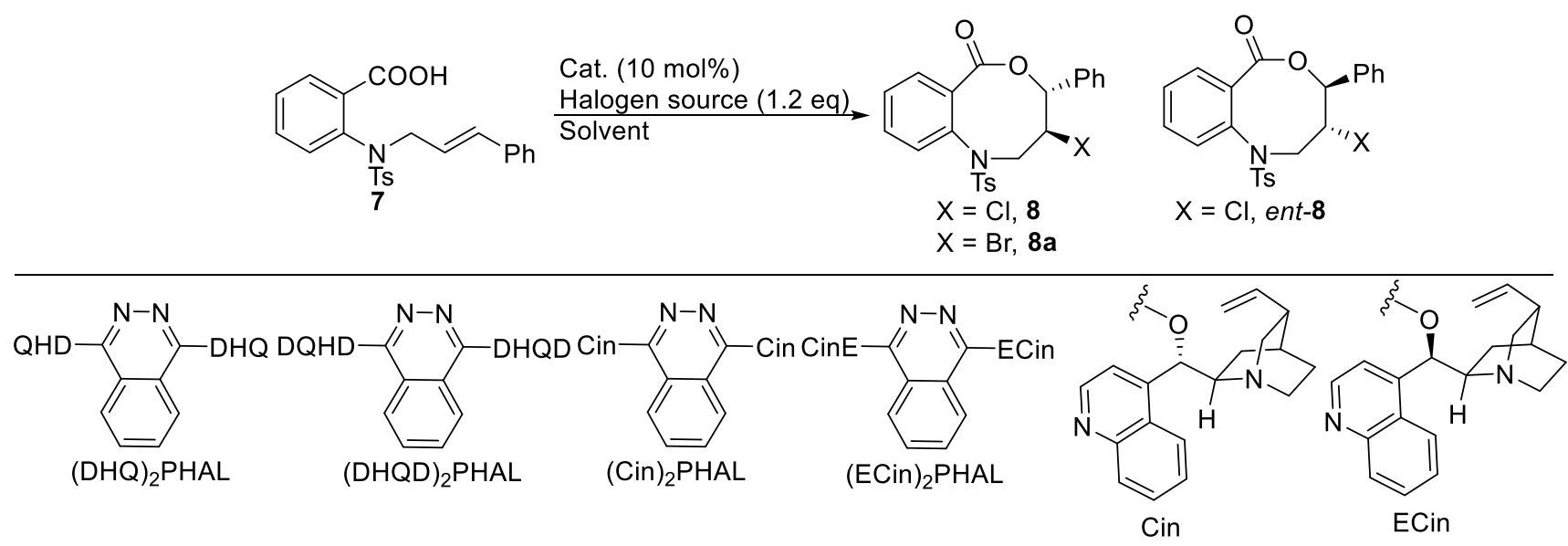

\begin{tabular}{llllllll}
\hline Entry & Halogen Source & Cat. & Solvent & Temp. & Yield (\%) & $\mathbf{8}(\mathrm{ee} \%)$ & $\mathbf{8 a}(\mathrm{ee} \%)$ \\
\hline 1 & $\mathrm{DCDMH}$ & (DHQD $)_{2}$ PHAL & PhMe & rt & 90 & 55
\end{tabular}




\begin{tabular}{|c|c|c|c|c|c|c|c|}
\hline 2 & $\mathrm{DCDMH}$ & $(\mathrm{DHQ})_{2} \mathrm{PHAL}$ & PhMe & rt & 91 & -46 & \\
\hline 3 & DCDMH & $(\mathrm{Cin})_{2} \mathrm{PHAL}$ & PhMe & rt & 92 & 57 & \\
\hline 4 & $\mathrm{DCDMH}$ & $(\mathrm{ECin})_{2} \mathrm{PHAL}$ & PhMe & rt & 90 & 60 & \\
\hline 5 & DCDMH & $(\mathrm{ECin})_{2} \mathrm{PHAL}$ & $\mathrm{CH}_{2} \mathrm{Cl}_{2}$ & rt & 95 & 71 & \\
\hline 6 & DCDMH & $(\mathrm{ECin})_{2} \mathrm{PHAL}$ & $\mathrm{CH}_{2} \mathrm{Cl}_{2}$ & $-10^{\circ} \mathrm{C}$ & 95 & 91 & \\
\hline 7 & $\mathrm{DCDMH}$ & $(\mathrm{ECin})_{2} \mathrm{PHAL}$ & $\mathrm{CH}_{2} \mathrm{Cl}_{2}$ & $-20^{\circ} \mathrm{C}$ & 95 & 91 & \\
\hline 8 & DCDMH & $(\mathrm{ECin})_{2} \mathrm{PHAL}$ & $\mathrm{CH}_{2} \mathrm{Cl}_{2}$ & $-30{ }^{\circ} \mathrm{C}$ & 93 & 85 & \\
\hline $9^{[\mathrm{b}]}$ & DCDPH & $(\mathrm{ECin})_{2} \mathrm{PHAL}$ & $\mathrm{CH}_{2} \mathrm{Cl}_{2}$ & $-20^{\circ} \mathrm{C}$ & 90 & 74 & \\
\hline 10 & NCS & $(\mathrm{ECin})_{2} \mathrm{PHAL}$ & $\mathrm{CH}_{2} \mathrm{Cl}_{2}$ & rt & $<5 \%$ & - & \\
\hline 11 & NBS & $(\mathrm{ECin})_{2} \mathrm{PHAL}$ & $\mathrm{CH}_{2} \mathrm{Cl}_{2}$ & $-30^{\circ} \mathrm{C}$ & 96 & - & 20 \\
\hline 12 & NBS & $(\mathrm{ECin})_{2} \mathrm{PHAL}$ & PhMe & $\mathrm{rt}$ & 95 & - & 24 \\
\hline $13^{[c]}$ & DCDMH & $(\text { ECin })_{2}$ PHAL & $\mathrm{CH}_{2} \mathrm{Cl}_{2}$ & $-20^{\circ} \mathrm{C}$ & 93 & 91 & \\
\hline $14^{[\mathrm{c}]}$ & DCDMH & $(\mathrm{ECin})_{2} \mathrm{PHAL}$ & $\mathrm{CH}_{2} \mathrm{Cl}_{2}$ & $-10^{\circ} \mathrm{C}$ & 94 & 89 & \\
\hline
\end{tabular}

[a] Reactions were carried out with substrate $8(0.1 \mathrm{mmol})$, Cat. $(0.01 \mathrm{mmol})$, and halogen source $(0.12 \mathrm{mmol})$ in solvent $(8 \mathrm{~mL})$. The yield was isolated yield and the ee was determined by chiral HPLC. [b] 1,3-dichloro-5,5-diphenyl-hydantoin (DCDPH) was used as halogen source. [c] The amount of (ECin) $)_{2}$ PHAL was $0.005 \mathrm{mmol}$.

NBS

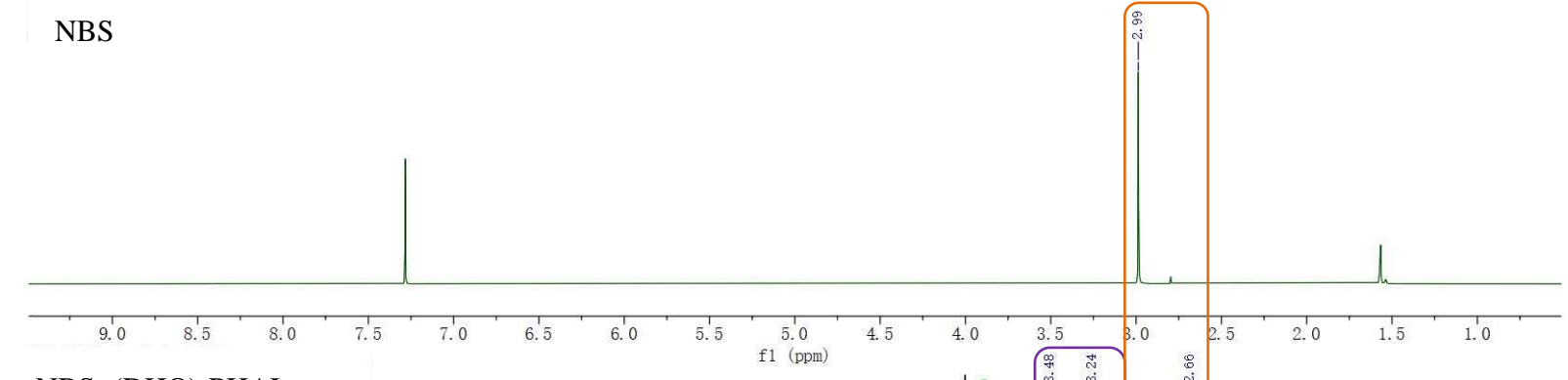
$\mathrm{NBS}+(\mathrm{DHQ})_{2} \mathrm{PHAL}$

$(2: 1)$

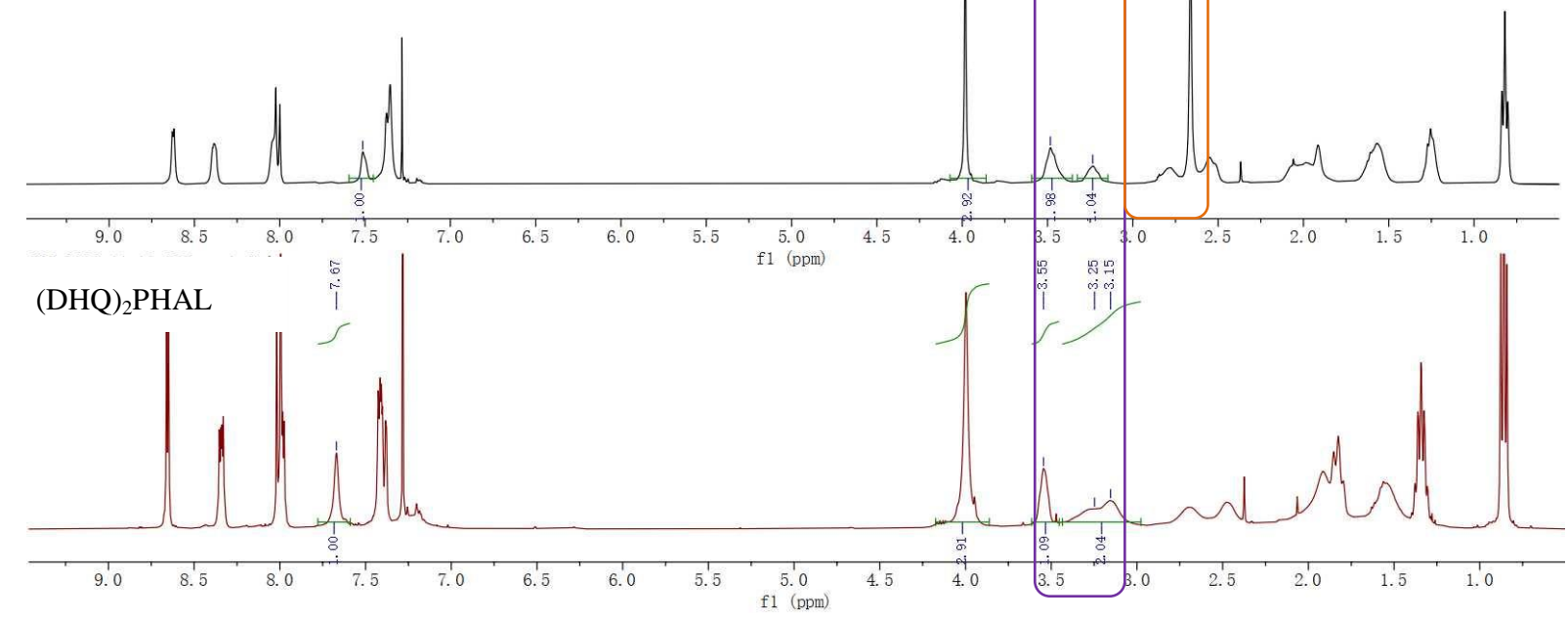

Figure 1. ${ }^{1} \mathrm{H}-\mathrm{NMR}$ analysis of $\mathrm{NBS}$ and $(\mathrm{DHQ})_{2} \mathrm{PHAL}$ 


\section{Evaluation of antibacterial activity.}

The compounds were diluted in $10 \%$ DMSO and $2 \mu \mathrm{L}$ of the dilution was added to a $200 \mu 1$ reaction so that the final concentration of DMSO is $0.1 \%$ in all of reactions. Minimum inhibitory concentrations (MIC) for compounds were determined following Luria-Bertani broth dilution guidelines . MIC values were determined as the lowest concentration of inhibitor to prevent visible Staphylococcus aureus growth after $18 \mathrm{~h}$ of incubation at $37{ }^{\circ} \mathrm{C}$. $\mathrm{OD}_{550 \mathrm{~nm}}$ absorbance signal was measured using a multi-well spectrophotometer (Molecular Devices SpectraMax M5 microplate reader).

The compounds were diluted in $10 \%$ DMSO and $2 \mu \mathrm{L}$ of the dilution was added to a $200 \mu 1$ reaction so that the final concentration of DMSO is $0.1 \%$ in all of reactions. Minimum inhibitory concentrations (MIC) for compounds were determined following Luria-Bertani broth dilution guidelines. MIC values were determined as the lowest concentration of inhibitor to prevent visible Staphylococcus aureus (MRSA) growth after $18 \mathrm{~h}$ of incubation at $37{ }^{\circ} \mathrm{C}$. $\mathrm{OD}_{550 \mathrm{~nm}}$ absorbance signal was measured using a multi-well spectrophotometer (Molecular Devices SpectraMax M5 microplate reader).

Staphylococcus aureus MIC results table

\begin{tabular}{|c|c|}
\hline Compound & MIC \\
\hline 2a & $1000 \mu \mathrm{g} / \mathrm{ml}$ \\
\hline $\mathbf{2 d}$ & $1000 \mu \mathrm{g} / \mathrm{ml}$ \\
\hline $\mathbf{9}$ & $10 \mu \mathrm{g} / \mathrm{ml}, 27.6 \mu \mathrm{M}$ \\
\hline$e n t-9$ & $6 \mu \mathrm{g} / \mathrm{ml}, 16.5 \mu \mathrm{M}$ \\
\hline Benzylpenicilin sodium & $0.003 \mu \mathrm{g} / \mathrm{ml}, 9 \mathrm{nM}$ \\
\hline
\end{tabular}

Staphylococcus aureus (MRSA) MIC results table

\begin{tabular}{|c|c|}
\hline Compound & MIC \\
\hline $\mathbf{2 a}$ & $1000 \mu \mathrm{g} / \mathrm{ml}$ \\
\hline $\mathbf{2 d}$ & $1000 \mu \mathrm{g} / \mathrm{ml}$ \\
\hline $\mathbf{9}$ & $10 \mu \mathrm{g} / \mathrm{ml}, 27.6 \mu \mathrm{M}$ \\
\hline$e n t-9$ & $6 \mu \mathrm{g} / \mathrm{ml}, 16.5 \mu \mathrm{M}$ \\
\hline Benzylpenicilin sodium & $0.1 \mu \mathrm{g} / \mathrm{ml}, 0.3 \mu \mathrm{M}$ \\
\hline
\end{tabular}




\section{SYNTHESIS OF CATALYSTS}

\section{Synthetic Procedure of (Cin) $)_{2}$ PHAL and (ECin) $)_{2}$ PHAL.}<smiles>C=CC1CC2CCN1CC2[C@H](O)c1ccnc2ccccc12</smiles>

$\mathrm{CinH}$<smiles>Brc1nnc(Br)c2ccccc12</smiles><smiles>CC(C)c1nnc(C(C)C)c2ccccc12</smiles>

$(\mathrm{Cin})_{2} \mathrm{PHAL}$

To a solution of cinchonine (588.0 mg, $2.0 \mathrm{mmol}, 1.0$ equiv) in DMF (5.0 mL) was added $\mathrm{NaH}$ ( $80.0 \mathrm{mg}, 2.0 \mathrm{mmol}, 1.0$ equiv) at room temperature. After stirring the reaction mixture for $10 \mathrm{~min}$, 1,4-dibromophthalazine ( $286.0 \mathrm{mg}, 1.0 \mathrm{mmol}, 0.5$ equiv.) was added. The mixture was then stirred for $72 \mathrm{~h}$ at $65^{\circ} \mathrm{C}$. Then water and EtOAc were added. The organic layer was washed with water, and dried over magnesium sulfate. Concentration of the organic layer offered the crude product that was further purified by flash column chromatography (hexane/EtOAc) to give the corresponding (Cin) $)_{2}$ PHAL $498.4 \mathrm{mg}, 70 \%$ as white solid; ${ }^{1} \mathrm{H}$ NMR $\left(\mathrm{CDCl}_{3}, 400 \mathrm{MHz}\right): \delta 8.82-7.50(\mathrm{~m}, 16 \mathrm{H}), 7.13$ $(\mathrm{d}, J=8.0 \mathrm{~Hz}, 2 \mathrm{H}), 5.97-5.89(\mathrm{~m}, 2 \mathrm{H}), 5.00-4.96(\mathrm{~m}, 4 \mathrm{H}), 3.49-1.52(\mathrm{~m}, 22 \mathrm{H}) ;{ }^{13} \mathrm{C} \mathrm{NMR}\left(\mathrm{CDCl}_{3}\right.$, $100 \mathrm{MHz}): \delta 156.5,149.9,148.6,146.3,140.3,132.2,130.3,129.0,126.6,126.4,124.2,123.1$, 122.5, 118.6, 114.7, 60.5, 49.9, 49.4, 39.7, 27.8, 26.4, 23.5; HRMS (TOF) m/z: $[\mathrm{M}+\mathrm{H}]^{+}$Calcd for $\mathrm{C}_{46} \mathrm{H}_{47} \mathrm{~N}_{6} \mathrm{O}_{2}$ 716.3391; found 716.3392.<smiles>C=CC1CC2CCN1C2[C@H](O)c1ccnc2ccccc12</smiles>

$\mathrm{ECinH}$

To a solution of epi-cinchonine $(588.0 \mathrm{mg}, 2.0 \mathrm{mmol}, 1.0$ equiv) in DMF (5.0 mL) was added $\mathrm{NaH}(80.0 \mathrm{mg}, 2.0 \mathrm{mmol}, 1.0$ equiv) at room temperature. After stirring the reaction mixture for 10 min, 1,4-dibromophthalazine (286.0 $\mathrm{mg}, 1.0 \mathrm{mmol}, 0.5$ equiv.) was added. The mixture was then stirred for $72 \mathrm{~h}$ at $65^{\circ} \mathrm{C}$. Then water and EtOAc were added. The organic layer was washed with water, and dried over magnesium sulfate. Concentration of the organic layer offered the crude product that was further purified by flash column chromatography (hexane/EtOAc) to give (ECin) $)_{2}$ PHAL $562.5 \mathrm{mg}, 79 \%$ as white solid; ${ }^{1} \mathrm{H} \mathrm{NMR}\left(\mathrm{CDCl}_{3}, 400 \mathrm{MHz}\right): \delta 8.82-7.51(\mathrm{~m}, 16 \mathrm{H})$, $7.13(\mathrm{~d}, J=8.0 \mathrm{~Hz}, 2 \mathrm{H}), 5.84-5.76(\mathrm{~m}, 2 \mathrm{H}), 4.98-4.92(\mathrm{~m}, 4 \mathrm{H}), 3.57-1.45(\mathrm{~m}, 22 \mathrm{H}) ;{ }^{13} \mathrm{C} \mathrm{NMR}$ $\left(\mathrm{CDCl}_{3}, 100 \mathrm{MHz}\right): \delta 156.5,149.9,148.7,146.1,141.9,132.5,130.3,129.1,126.6,126.2,124.2$, 122.9, 122.5, 118.7, 114.4, 60.6, 56.9, 42.6, 39.8, 27.9, 27.8, 23.9; HRMS (TOF) m/z: $[\mathrm{M}+\mathrm{H}]^{+}$Calcd for $\mathrm{C}_{46} \mathrm{H}_{47} \mathrm{~N}_{6} \mathrm{O}_{2} 716.3391$; found 716.3393 .

\section{SYNTHESIS OF ALKENOIC ACID SUBSTRATES AND ASYMMETRIC HALOLACTONIZATION REACTIONS}

General Procedure for the Synthesis of Alkenoic Acid Substrate. 


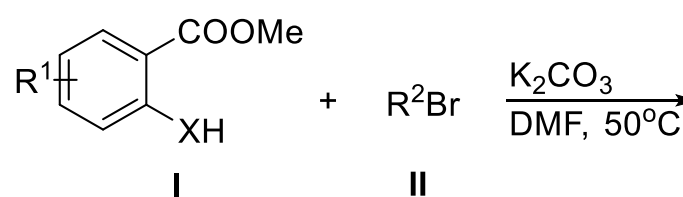

$\mathrm{X}=\mathrm{O}, \mathrm{NSO}_{2} \mathrm{R}$<smiles>[R][X]C1=CC=C[R1]C=C1C(=O)OC</smiles>

III

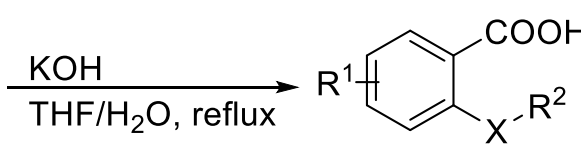

1a-s, 5a-b, 7

To a solution of $\mathbf{I}$ (2.0 mmol, 1.0 equiv) and $\mathrm{K}_{2} \mathrm{CO}_{3}(276.0 \mathrm{mg}, 2.0 \mathrm{mmol}, 1.0$ equiv) in DMF (5.0 $\mathrm{mL}$ ) was added II ( $2.0 \mathrm{mmol}, 1.0$ equiv) at room temperature. The mixture was then stirred for $24 \mathrm{~h}$ at $50^{\circ} \mathrm{C}$. Water $(20.0 \mathrm{~mL})$ and EtOAc $(30.0 \mathrm{~mL})$ were added. The organic layer was washed with water, and dried over magnesium sulfate. Concentration of the organic layer offered the crude product that was further purified by flash column chromatography (hexane/EtOAc) to give the corresponding alkenoic ester III.

To a solution of III (1.0 mmol, 1.0 equiv) in THF/ $\mathrm{H}_{2} \mathrm{O}(\mathrm{v} / \mathrm{v}=5.0 \mathrm{~mL} / 5.0 \mathrm{~mL})$ was added $\mathrm{KOH}$ (560.0 mg, $10.0 \mathrm{mmol}, 10.0$ equiv) at room temperature. The mixture was then stirred for $24 \mathrm{~h}$ at $75^{\circ} \mathrm{C}$. After evaporation of THF, acetic acid, water and EtOAc were added. The organic layer was washed with water, and dried over magnesium sulfate. Concentration of the organic layer offered the crude product that was further purified by flash column chromatography (hexane/EtOAc) to give the pure alkenoic acid $\mathbf{1 a}-\mathbf{s}, \mathbf{5 a}-\mathbf{b}$ and $\mathbf{7}$.

\section{Representative Procedure for the Synthesis of Alkenoic Acid Substrate 1a.}

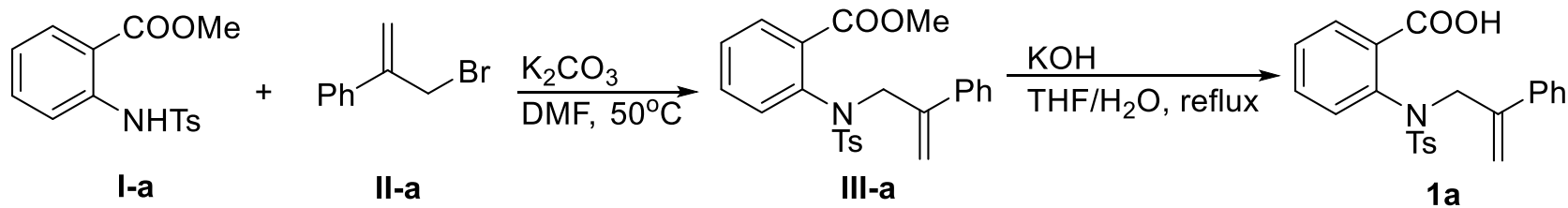

To a solution of $\mathbf{I - a}\left(610.0 \mathrm{mg}, 2.0 \mathrm{mmol}, 1.0\right.$ equiv) and $\mathrm{K}_{2} \mathrm{CO}_{3}(276.0 \mathrm{mg}, 2.0 \mathrm{mmol}, 1.0$ equiv) in DMF (5.0 mL) was added II-a (392.0 mg, $2.0 \mathrm{mmol}, 1.0$ equiv) at room temperature. The mixture was then stirred for $24 \mathrm{~h}$ at $50^{\circ} \mathrm{C}$. Water $(20 \mathrm{~mL})$ and EtOAc $(30.0 \mathrm{~mL})$ were added. The organic layer was washed with water, and dried over magnesium sulfate. Concentration of the organic layer offered the crude product that was further purified by flash column chromatography (hexane/EtOAc) to give the corresponding alkenoic ester III-a $766.2 \mathrm{mg}, 91 \%$, white solid; MP $126-127{ }^{\circ} \mathrm{C}$; ${ }^{1} \mathrm{H}$ NMR $\left(\mathrm{CDCl}_{3}, 400 \mathrm{MHz}\right): \delta 7.86-6.69(\mathrm{~m}, 13 \mathrm{H}), 5.31(\mathrm{~s}, 1 \mathrm{H}), 5.14(\mathrm{~s}, 1 \mathrm{H}), 4.83(\mathrm{~s}, 2 \mathrm{H}), 3.61(\mathrm{~s}, 3 \mathrm{H})$, $2.42(\mathrm{~s}, 3 \mathrm{H}) ;{ }^{13} \mathrm{C} \mathrm{NMR}\left(\mathrm{CDCl}_{3}, 100 \mathrm{MHz}\right): \delta 166.5,143.2,142.9,138.8,137.5,136.6,132.1,131.8$, 131.7, 131.5, 129.3, 128.3, 128.2, 127.8, 127.7, 126.6, 118.2, 54.8, 52.0, 21.5; HRMS (TOF) m/z: $[\mathrm{M}+\mathrm{H}]^{+}$Calcd for $\mathrm{C}_{24} \mathrm{H}_{24} \mathrm{NO}_{4} \mathrm{~S} 422.6350$; found 422.6351 .

To a solution of III-a (421.0 mg, $1.0 \mathrm{mmol}, 1.0$ equiv) in $\mathrm{THF} / \mathrm{H}_{2} \mathrm{O}(\mathrm{v} / \mathrm{v}=5.0 \mathrm{~mL} / 5.0 \mathrm{~mL})$ was added $\mathrm{KOH}(560.0 \mathrm{mg}, 10.0 \mathrm{mmol}, 10.0$ equiv) at room temperature. The mixture was then stirred for $24 \mathrm{~h}$ at $75^{\circ} \mathrm{C}$. After evaporation of THF, acetic acid, water and EtOAc were added. The organic layer was washed with water, and dried over magnesium sulfate. Concentration of the organic layer offered the crude product that was further purified by flash column chromatography (hexane/EtOAc) to give the corresponding alkenoic acid 1a $366.3 \mathrm{mg}$, 90\%, white solid; MP $234-235^{\circ} \mathrm{C} ;{ }^{1} \mathrm{H}$ NMR $\left(\mathrm{CDCl}_{3}, 400 \mathrm{MHz}\right): \delta 7.99-6.72(\mathrm{~m}, 13 \mathrm{H}), 5.34(\mathrm{~s}, 1 \mathrm{H}), 5.15(\mathrm{~s}, 1 \mathrm{H}), 4.84(\mathrm{~d}, J=12.0 \mathrm{~Hz}, 2 \mathrm{H}), 2.41$ $(\mathrm{s}, 3 \mathrm{H}) ;{ }^{13} \mathrm{C} \mathrm{NMR}\left(\mathrm{CDCl}_{3}, 100 \mathrm{MHz}\right): \delta 169.4,144.0,142.6,138.3,137.6,135.3,132.6,132.2,131.5$, 
131.3, 129.5, 128.5, 128.2, 128.1, 126.6, 118.5, 55.1, 21.6; HRMS (TOF) m/z: [M-H] $]^{-}$Calcd for $\mathrm{C}_{23} \mathrm{H}_{20} \mathrm{NO}_{4} \mathrm{~S} 406.8055$; found 406.8054 .<smiles>C=C(CN(c1ccccc1C(C)=O)S(=O)(=O)c1ccccc1)c1ccccc1</smiles>

III-b<smiles>C=C(CN(c1ccccc1C(=O)O)S(=O)(=O)c1ccccc1)c1ccccc1</smiles>

1b

1b. $341.0 \mathrm{mg}, 87 \%$, yellow solid (starting from $407.1 \mathrm{mg}$ of III-b); MP $220-221{ }^{\circ} \mathrm{C}$; ${ }^{1} \mathrm{H} \mathrm{NMR}$ $\left(\mathrm{CDCl}_{3}, 400 \mathrm{MHz}\right): \delta 7.99-6.73(\mathrm{~m}, 14 \mathrm{H}), 5.35(\mathrm{~s}, 1 \mathrm{H}), 5.18(\mathrm{~s}, 1 \mathrm{H}), 4.93(\mathrm{~d}, J=32.0 \mathrm{~Hz}, 2 \mathrm{H}) ;{ }^{13} \mathrm{C}$ NMR $\left(\mathrm{CDCl}_{3}, 100 \mathrm{MHz}\right): \delta 170.0,142.9,142.8,138.8,138.6,137.7,132.9,132.7,132.3,128.9$, 128.5, 128.4, 128.1, 127.9, 126.6, 118.4, 55.2; HRMS (TOF) m/z: $[\mathrm{M}-\mathrm{H}]^{-}$Calcd for $\mathrm{C}_{22} \mathrm{H}_{18} \mathrm{NO}_{4} \mathrm{~S}$ 392.6297; found 392.6298.<smiles>C=C(CN(c1ccccc1C(C)=O)S(=O)(=O)c1ccc(OC)cc1)c1ccccc1</smiles>

1c. $363.8 \mathrm{mg}, 86 \%$, yellow solid (starting from $437.1 \mathrm{mg}$ of III-c); MP $250-251{ }^{\circ} \mathrm{C}$; ${ }^{1} \mathrm{H}$ NMR $\left(\mathrm{CDCl}_{3}, 400 \mathrm{MHz}\right): \delta 7.99-6.75(\mathrm{~m}, 13 \mathrm{H}), 5.34(\mathrm{~s}, 1 \mathrm{H}), 5.13(\mathrm{~s}, 1 \mathrm{H}), 4.85(\mathrm{~d}, J=36.0 \mathrm{~Hz}, 2 \mathrm{H}), 3.84$, $(\mathrm{s}, 3 \mathrm{H}) ;{ }^{13} \mathrm{C} \mathrm{NMR}\left(\mathrm{CDCl}_{3}, 100 \mathrm{MHz}\right): \delta 168.9,163.3,142.6,142.5,138.3,137.6,132.5,131.2,131.1$, 130.3, 129.6, 128.5, 128.2, 126.8, 118.5, 114.1, 56.6, 55.2; HRMS (TOF) m/z: $[\mathrm{M}-\mathrm{H}]^{-}$Calcd for $\mathrm{C}_{23} \mathrm{H}_{20} \mathrm{NO}_{5} \mathrm{~S} 422.7035$; found 422.7034 .<smiles>C=C(CN(c1ccccc1)c1ccccc1)c1ccccc1</smiles>

III-d<smiles>C=C(CN([N+]#N)c1ccccc1C(=O)O)c1ccccc1</smiles>

$1 d$

1d. $372.3 \mathrm{mg}, 85 \%$, yellow solid (starting from $452.2 \mathrm{mg}$ of III-d); MP $283-285^{\circ} \mathrm{C}$; ${ }^{1} \mathrm{H}$ NMR $\left(\mathrm{CDCl}_{3}, 400 \mathrm{MHz}\right): \delta 8.22-6.69(\mathrm{~m}, 13 \mathrm{H}), 5.33(\mathrm{~s}, 1 \mathrm{H}), 5.28(\mathrm{~d}, J=16.0 \mathrm{~Hz}, 1 \mathrm{H}), 5.14(\mathrm{~s}, 1 \mathrm{H}), 4.61$ $(\mathrm{d}, J=16.0 \mathrm{~Hz}, 1 \mathrm{H}) ;{ }^{13} \mathrm{C} \mathrm{NMR}\left(\mathrm{CDCl}_{3}, 100 \mathrm{MHz}\right): \delta 170.4,149.8,145.5,142.9,138.6,137.2,133.8$, 133.3, 132.5, 129.4, 129.1, 128.7, 128.5, 128.2, 126.8, 123.9, 119.1, 56.5; HRMS (TOF) m/z: [M$\mathrm{H}]^{-}$Calcd for $\mathrm{C}_{22} \mathrm{H}_{17} \mathrm{~N}_{2} \mathrm{O}_{6} \mathrm{~S} \quad 437.5217$; found 437.5218.<smiles>C=C(CN(c1ccccc1C(=O)O)c1ccccc1C(=O)O)c1ccccc1</smiles>

1e. $297.9 \mathrm{mg}, 90 \%$, white solid (starting from $345.3 \mathrm{mg}$ of III-e); MP $196-197{ }^{\circ} \mathrm{C} ;{ }^{1} \mathrm{H} \mathrm{NMR}\left(\mathrm{CDCl}_{3}\right.$, $400 \mathrm{MHz}): \delta 8.08-6.97(\mathrm{~m}, 11 \mathrm{H}), 5.35(\mathrm{~s}, 1 \mathrm{H}), 5.17(\mathrm{~s}, 1 \mathrm{H}), 2.82(\mathrm{~s}, 3 \mathrm{H}) ;{ }^{13} \mathrm{C} \mathrm{NMR}\left(\mathrm{CDCl}_{3}, 100\right.$ 
MHz): $\delta 170.3,143.7,141.2,138.7,138.2,133.3,133.1,132.3,129.9,128.8,128.6,128.5,128.2$, 127.3, 126.9, 118.4, 110.3, 55.2, 40.2; HRMS (TOF) m/z: $[\mathrm{M}-\mathrm{H}]^{-}$Calcd for $\mathrm{C}_{17} \mathrm{H}_{16} \mathrm{NO}_{4} \mathrm{~S} 330.5184$; found 330.5186 .<smiles>C=C(CNc1ccccc1C(=O)OC)c1ccccc1C</smiles>

III-f<smiles>C=C(CN(C)c1ccccc1C(=O)O)c1ccccc1C</smiles>

$1 f$

1f. $373.8 \mathrm{mg}, 89 \%$, white solid (starting from $435.2 \mathrm{mg}$ of III-f); MP $239-240{ }^{\circ} \mathrm{C} ;{ }^{1} \mathrm{H} \mathrm{NMR}\left(\mathrm{CDCl}_{3}\right.$, $400 \mathrm{MHz}): \delta 9.81(\mathrm{~s}$, broad, $1 \mathrm{H}), 8.04-6.92(\mathrm{~m}, 12 \mathrm{H}), 5.61(\mathrm{~s}, 1 \mathrm{H}), 5.11(\mathrm{~s}, 1 \mathrm{H}), 4.70(\mathrm{~d}, J=36.0 \mathrm{~Hz}$, 2H), 2.39 (s, 3H), 2.12 (s, 3H); ${ }^{13} \mathrm{C} \mathrm{NMR}\left(\mathrm{CDCl}_{3}, 100 \mathrm{MHz}\right): \delta 169.9,144.2,143.8,139.5,138.7$, 135.5, 132.8, 132.3, 131.5, 130.5, 129.4, 128.8, 128.4, 128.0, 127.7, 125.7,118.8, 57.1, 21.5, 19.8; HRMS (TOF) m/z: [M-H] ${ }^{-}$Calcd for $\mathrm{C}_{24} \mathrm{H}_{22} \mathrm{NO}_{4} \mathrm{~S} 420.2965$; found 420.2966.

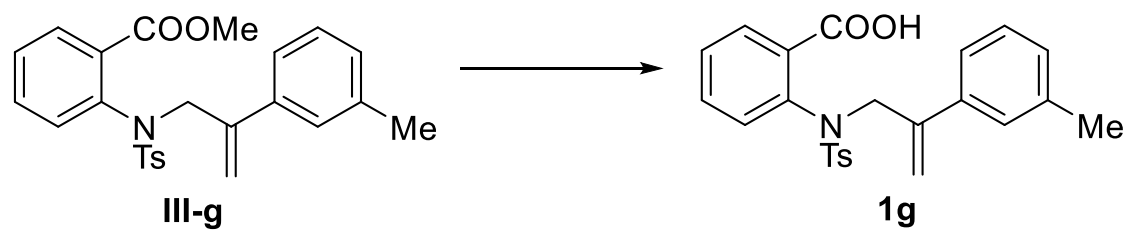

1g. $365.4 \mathrm{mg}, 87 \%$, white solid (starting from $435.2 \mathrm{mg}$ of III-g); MP $236-237^{\circ} \mathrm{C}$; ${ }^{1} \mathrm{H}$ NMR $\left(\mathrm{CDCl}_{3}, 400 \mathrm{MHz}\right): \delta 8.0-6.74(\mathrm{~m}, 12 \mathrm{H}), 5.32(\mathrm{~s}, 1 \mathrm{H}), 5.14(\mathrm{~s}, 1 \mathrm{H}), 4.81(\mathrm{~s}$, broad, $2 \mathrm{H}), 2.41(\mathrm{~s}, 3 \mathrm{H})$, $2.33(\mathrm{~s}, 3 \mathrm{H}) ;{ }^{13} \mathrm{C} \mathrm{NMR}\left(\mathrm{CDCl}_{3}, 100 \mathrm{MHz}\right): \delta 169.3,143.9,142.7,138.3,138.0,137.7,135.3,132.6$, $131.5,131.2$, 129.5, 128.9,128.5, 128.4, 128.1, 127.3, 123.7,118.2, 55.3, 21.6, 21.5; HRMS (TOF) $\mathrm{m} / \mathrm{z}:[\mathrm{M}-\mathrm{H}]^{-}$Calcd for $\mathrm{C}_{24} \mathrm{H}_{22} \mathrm{NO}_{4} \mathrm{~S}$ 420.2965; found 420.2967 .<smiles>C=C(CN(C)c1ccccc1C(=O)OCc1ccccc1)c1ccc(C)cc1</smiles>

1h. $357.0 \mathrm{mg}, 85 \%$, white solid (starting from $435.1 \mathrm{mg}$ of III-h); MP $241-242{ }^{\circ} \mathrm{C}$; ${ }^{1} \mathrm{H}$ NMR $\left(\mathrm{CDCl}_{3}, 400 \mathrm{MHz}\right): \delta$ 7.99-6.75 (m, 12H), $5.30(\mathrm{~s}, 1 \mathrm{H}), 5.08(\mathrm{~s}, 1 \mathrm{H}), 4.82(\mathrm{~s}, 2 \mathrm{H}), 2.41(\mathrm{~s}, 3 \mathrm{H}), 2.36$ $(\mathrm{s}, 3 \mathrm{H}) ;{ }^{13} \mathrm{C} \mathrm{NMR}\left(\mathrm{CDCl}_{3}, 100 \mathrm{MHz}\right): \delta 169.3,143.9,142.4,138.0,137.7,135.5,135.4,132.6,132.2$, 131.6,131.2, 129.5, 129.1, 128.5, 128.1, 126.4, 117.1, 55.1, 21.6, 21.2; HRMS (TOF) m/z: $[\mathrm{M}-\mathrm{H}]^{-}$ Calcd for $\mathrm{C}_{24} \mathrm{H}_{22} \mathrm{NO}_{4} \mathrm{~S} 420.2965$; found 420.2964 .<smiles>C=C(CN(C)c1ccccc1C(=O)O)c1cccc(OC)c1</smiles><smiles>C=C(CN(C)c1ccccc1C(=O)O)c1cccc(OC)c1</smiles>

1i. $375.8 \mathrm{mg}, 86 \%$, white solid (starting from $451.3 \mathrm{mg}$ of III-i); MP $237-238^{\circ} \mathrm{C} ;{ }^{1} \mathrm{H}$ NMR $\left(\mathrm{CDCl}_{3}\right.$, $300 \mathrm{MHz}): \delta 8.00-6.74(\mathrm{~m}, 12 \mathrm{H}), 5.36(\mathrm{~s}, 1 \mathrm{H}), 5.14(\mathrm{~s}, 1 \mathrm{H}), 4.82(\mathrm{~s}, 2 \mathrm{H}), 3.83(\mathrm{~s}, 3 \mathrm{H}), 2.40(\mathrm{~s}, 3 \mathrm{H})$; ${ }^{13} \mathrm{C} \mathrm{NMR}\left(\mathrm{CDCl}_{3}, 75 \mathrm{MHz}\right): \delta 169.6,159.6,143.9,142.5,139.8,137.7,135.4,132.6,132.2,131.6$, 
131.2, 129.4, 128.5, 128.0, 119.0, 118.6, 114.1, 112.1, 55.3, 21.6; HRMS (TOF) m/z: $[\mathrm{M}-\mathrm{H}]^{-}$ Calcd for $\mathrm{C}_{24} \mathrm{H}_{22} \mathrm{NO}_{5} \mathrm{~S} \quad 436.5177$; found 436.5178 .<smiles>C=C(CN(C)c1ccccc1C(=O)O)c1cccc(Br)c1</smiles>

1j. $426.8 \mathrm{mg}, 88 \%$, white solid (starting from $499.5 \mathrm{mg}$ of III-j); MP $261-262{ }^{\circ} \mathrm{C} ;{ }^{1} \mathrm{H} \mathrm{NMR}\left(\mathrm{CDCl}_{3}\right.$, $400 \mathrm{MHz}): \delta$ 7.99-6.73 (m, 12H), $5.34(\mathrm{~s}, 1 \mathrm{H}), 5.19(\mathrm{~s}, 1 \mathrm{H}), 4.78(\mathrm{~s}, 2 \mathrm{H}), 2.41(\mathrm{~s}, 3 \mathrm{H}) ;{ }^{13} \mathrm{C} \mathrm{NMR}$ $\left(\mathrm{CDCl}_{3}, 100 \mathrm{MHz}\right): \delta 169.5,144.0,141.7,140.7,137.7,135.5,132.7,132.4,131.6,131.2$, 130.0,129.7,129.5, 128.6, 128.0, 125.3,122.5, 119.6, 55.1, 21.6; HRMS (TOF) m/z: $[\mathrm{M}-\mathrm{H}]^{-} \mathrm{Calcd}$ for $\mathrm{C}_{23} \mathrm{H}_{19} \mathrm{NO}_{4} \mathrm{SBr} 484.3719$; found 484.2717.<smiles>C=C(CN(C)c1ccccc1C(=O)O)c1cccc2ccccc12</smiles>

III-k

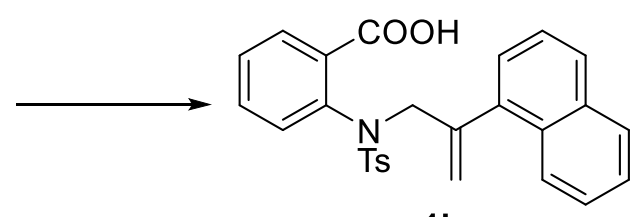

$1 k$

1k. $411.3 \mathrm{mg}$, 90\%, white solid (starting from $471.4 \mathrm{mg}$ of III-k); MP $268-269^{\circ} \mathrm{C}$; ${ }^{1} \mathrm{H}$ NMR $\left(\mathrm{CDCl}_{3}, 400 \mathrm{MHz}\right): \delta 8.01-6.97(\mathrm{~m}, 15 \mathrm{H}), 5.89(\mathrm{~s}, 1 \mathrm{H}), 5.35(\mathrm{~s}, 1 \mathrm{H}), 4.88(\mathrm{~d}, J=56.0 \mathrm{~Hz}, 2 \mathrm{H}), 2.32$ $(\mathrm{s}, 3 \mathrm{H}) ;{ }^{13} \mathrm{C} \mathrm{NMR}\left(\mathrm{CDCl}_{3}, 100 \mathrm{MHz}\right): \delta 170.0,143.7,143.0,138.8,138.0,135.6,133.8,132.9,131.8$, $131.3,130.4,129.4,128.5,128.4,128.0,127.8,126.1,125.8,125.2,120.0,57.9,21.5$; HRMS (TOF) $\mathrm{m} / \mathrm{z}:[\mathrm{M}-\mathrm{H}]^{-}$Calcd for $\mathrm{C}_{27} \mathrm{H}_{22} \mathrm{NO}_{4} \mathrm{~S} 456.2974$; found 456.2975 .<smiles>C=C(CN([As])c1ccccc1C(C)=O)c1ccc2ccccc2c1</smiles>

III-I<smiles>C=C(CN(C)c1ccccc1C(=O)O)c1ccc2ccccc2c1</smiles>

11

11. $406.7 \mathrm{mg}, 89 \%$, white solid (starting from $471.2 \mathrm{mg}$ of III-I); MP $233-234{ }^{\circ} \mathrm{C} ;{ }^{1} \mathrm{H} \mathrm{NMR}\left(\mathrm{CDCl}_{3}\right.$, $400 \mathrm{MHz}): \delta$ 7.96-6.73 (m, 15H), $5.48(\mathrm{~s}, 1 \mathrm{H}), 5.21(\mathrm{~s}, 1 \mathrm{H}), 4.91(\mathrm{~s}, 2 \mathrm{H}), 2.37(\mathrm{~s}, 3 \mathrm{H}) ;{ }^{13} \mathrm{C} \mathrm{NMR}$ $\left(\mathrm{CDCl}_{3}, 100 \mathrm{MHz}\right): \delta 169.0,167.8,143.9,142.4,137.7,135.6,135.5,133.3,133.1,132.6,132.2$, 131.6, 129.5, 128.5, 128.4, 128.0, 127.5, 126.2, 125.7, 124.6, 119.1, 55.3, 21.5; HRMS (TOF) m/z: $[\mathrm{M}-\mathrm{H}]^{-}$Calcd for $\mathrm{C}_{27} \mathrm{H}_{22} \mathrm{NO}_{4} \mathrm{~S} \quad 456.2974$; found 456.2973 .

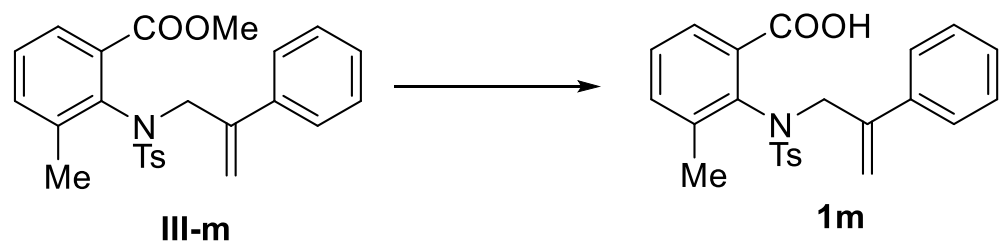

1m. $366.3 \mathrm{mg}, 87 \%$, white solid (starting from $435.4 \mathrm{mg}$ of III-m); MP $245-246{ }^{\circ} \mathrm{C}$; ${ }^{1} \mathrm{H}$ NMR $\left(\mathrm{CDCl}_{3}, 400 \mathrm{MHz}\right): \delta 7.79-7.21(\mathrm{~m}, 12 \mathrm{H}), 5.37(\mathrm{~s}, 1 \mathrm{H}), 5.16(\mathrm{~s}, 1 \mathrm{H}), 5.21\left(\mathrm{dd}, J_{1}=20.0 \mathrm{~Hz}, J_{2}=\right.$ $200.4 \mathrm{~Hz}, 2 \mathrm{H}), 2.36$ (s, 3H), 1.78 (s, 1H); ${ }^{13} \mathrm{C} \mathrm{NMR}\left(\mathrm{CDCl}_{3}, 100 \mathrm{MHz}\right): \delta 170.4,143.3,143.2,139.1$, 137.6, 136.8, 136.0,129.8, 129.3, 128.2, 128.1, 127.8, 126.6, 119.7, 54.8, 21.6,19.2; HRMS (TOF) $\mathrm{m} / \mathrm{z}:[\mathrm{M}-\mathrm{H}]^{-}$Calcd for $\mathrm{C}_{24} \mathrm{H}_{23} \mathrm{NO}_{4} \mathrm{~S}$ 420.5177; found 420.5176 . 
<smiles>C=CC(=O)N(CC(=O)c1ccccc1)c1cc(C)ccc1C(=O)O</smiles>

1n. $353.6 \mathrm{mg}, 84 \%$, white solid (starting from $435.1 \mathrm{mg}$ of III-n); MP $259-260{ }^{\circ} \mathrm{C}$; ${ }^{1} \mathrm{H}$ NMR $\left(\mathrm{CDCl}_{3}, 400 \mathrm{MHz}\right): \delta 7.88-6.49(\mathrm{~m}, 12 \mathrm{H}), 5.33(\mathrm{~s}, 1 \mathrm{H}), 5.17(\mathrm{~s}, 1 \mathrm{H}), 4.85(\mathrm{~d}, J=24.0 \mathrm{~Hz}, 2 \mathrm{H}), 2.39$ $(\mathrm{s}, 3 \mathrm{H}), 2.21(\mathrm{~s}, 1 \mathrm{H}) ;{ }^{13} \mathrm{C} \mathrm{NMR}\left(\mathrm{CDCl}_{3}, 100 \mathrm{MHz}\right): \delta 169.5,143.7,143.1,138.6,137.8,135.9,133.2$, 132.1, 129.4, 129.2, 128.3, 128.0, 127.6, 126.7, 118.1, 55.1, 21.6, 21.2; HRMS (TOF) m/z: [M-H] Calcd for $\mathrm{C}_{24} \mathrm{H}_{22} \mathrm{NO}_{4} \mathrm{~S} 420.5177$; found 420.5179 .<smiles>COC(=O)c1cc(C)ccc1N(CC(=O)c1ccccc1)[13CH]=[18O]</smiles>

1o. $374.7 \mathrm{mg}, 89 \%$, white solid (starting from $435.2 \mathrm{mg}$ of III-o); MP $255-256{ }^{\circ} \mathrm{C}$; ${ }^{1} \mathrm{H}$ NMR $\left(\mathrm{CDCl}_{3}, 400 \mathrm{MHz}\right): \delta$ 7.78-6.61 (m, 12H), $5.35(\mathrm{~s}, 1 \mathrm{H}), 5.15(\mathrm{~s}, 1 \mathrm{H}), 4.83(\mathrm{~s}, 2 \mathrm{H}), 2.41(\mathrm{~s}, 3 \mathrm{H}), 2.39$ $(\mathrm{s}, 1 \mathrm{H}) ;{ }^{13} \mathrm{C} \mathrm{NMR}\left(\mathrm{CDCl}_{3}, 100 \mathrm{MHz}\right): \delta 169.7,143.9,142.7,138.8,138.5,135.5,135.0,133.3,132.7$, 131.4, 130.9, 129.5, 128.4, 128.1, 126.6, 118.3, 56.2, 21.6, 21.0; HRMS (TOF) m/z: [M-H] ${ }^{-} \mathrm{Calcd}$ for $\mathrm{C}_{24} \mathrm{H}_{22} \mathrm{NO}_{4} \mathrm{~S} 420.5177$; found 420.5178 .<smiles>C=C(CN([18OH])c1cc(OC)ccc1C(=O)OC)c1ccccc1</smiles>

1p. $401.3 \mathrm{mg}$, 91\%, white solid (starting from $437.5 \mathrm{mg}$ of III-p); MP $287-288^{\circ} \mathrm{C}$; ${ }^{1} \mathrm{H}$ NMR $\left(\mathrm{CDCl}_{3}, 400 \mathrm{MHz}\right): \delta 7.97-6.17(\mathrm{~m}, 12 \mathrm{H}), 5.33(\mathrm{~s}, 1 \mathrm{H}), 5.14(\mathrm{~s}, 1 \mathrm{H}), 4.82(\mathrm{~s}, 2 \mathrm{H}), 3.60(\mathrm{~s}, 3 \mathrm{H}), 2.41$ $(\mathrm{s}, 3 \mathrm{H}) ;{ }^{13} \mathrm{C} \mathrm{NMR}\left(\mathrm{CDCl}_{3}, 100 \mathrm{MHz}\right): \delta 167.9,162.6,143.9,142.6,139.7,138.3,135.5,134.0,129.5$, 128.5, 128.2, 126.6, 122.5, 118.5, 117.1, 114.5, 55.4, 55.0, 21.6; HRMS (TOF) m/z: $[\mathrm{M}-\mathrm{H}]^{-} \mathrm{Calcd}$ for $\mathrm{C}_{24} \mathrm{H}_{22} \mathrm{NO}_{5} \mathrm{~S} 436.7418$; found 436.7419 .<smiles>C=C(CN(C)c1cc(F)ccc1C(=O)O)c1ccccc1</smiles><smiles>CN(CC(=O)c1ccccc1)c1cc(F)ccc1C(=O)O</smiles>

1q. $382.5 \mathrm{mg}$, 90\%, white solid (starting from $439.3 \mathrm{mg}$ of III-q); MP $253-254{ }^{\circ} \mathrm{C}$; ${ }^{1} \mathrm{H}$ NMR $\left(\mathrm{CDCl}_{3}, 400 \mathrm{MHz}\right): \delta 8.04-6.43(\mathrm{~m}, 12 \mathrm{H}), 5.36(\mathrm{~s}, 1 \mathrm{H}), 5.18(\mathrm{~s}, 1 \mathrm{H}), 4.82(\mathrm{~s}, 2 \mathrm{H}), 2.42(\mathrm{~s}, 3 \mathrm{H}) ;{ }^{13} \mathrm{C}$ $\operatorname{NMR}\left(\mathrm{CDCl}_{3}, 100 \mathrm{MHz}\right): \delta 169.0,165.7(\mathrm{~d}, J=102.0 \mathrm{~Hz}), 144.1,142.7,140.5,138.3,135.5,134.2$, 129.6, 128.5, 128.2, 128.0, 127.1, 126.6, 119.5 (d, $J=2.8 \mathrm{~Hz}), 118.5,115.7$ (d, $J=8.4 \mathrm{~Hz}), 56.0$, 21.6; HRMS (TOF) m/z: $[\mathrm{M}-\mathrm{H}]^{-}$Calcd for $\mathrm{C}_{23} \mathrm{H}_{19} \mathrm{FNO}_{4} \mathrm{~S} 424.7033$; found 424.7032 . 


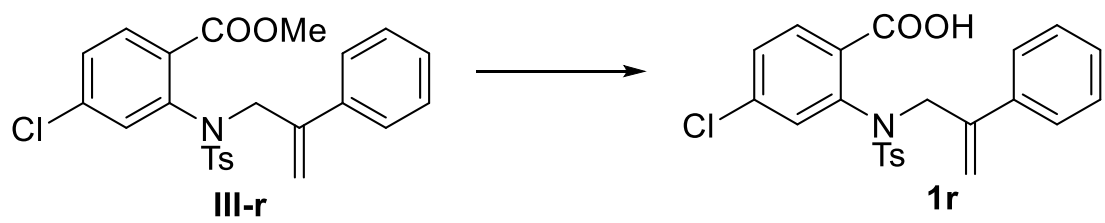

1r. $401.3 \mathrm{mg}, 91 \%$, white solid (starting from $455.6 \mathrm{mg}$ of III-r); MP $269-270{ }^{\circ} \mathrm{C} ;{ }^{1} \mathrm{H} \mathrm{NMR}\left(\mathrm{CDCl}_{3}\right.$, $400 \mathrm{MHz}): \delta$ 7.93-6.62 (m, 12H), $5.35(\mathrm{~s}, 1 \mathrm{H}), 5.16(\mathrm{~s}, 1 \mathrm{H}), 4.71(\mathrm{~s}, 2 \mathrm{H}), 2.43(\mathrm{~s}, 3 \mathrm{H}) ;{ }^{13} \mathrm{C} \mathrm{NMR}$ $\left(\mathrm{CDCl}_{3}, 100 \mathrm{MHz}\right): \delta 168.8,144.2,142.6,139.1,138.5,138.2,135.3,133.1,132.1,129.6,129.3$, 128.1, 128.5, 128.3, 128.0, 126.6, 118.6, 55.0, 21.6; HRMS (TOF) $\mathrm{m} / \mathrm{z}:[\mathrm{M}-\mathrm{H}]^{-}$Calcd for $\mathrm{C}_{23} \mathrm{H}_{19} \mathrm{ClNO}_{4} \mathrm{~S} 440.6015$; found 440.6014 .<smiles>C=C(CN([14CH3])c1cc(Br)ccc1C(=O)O)c1cccc(C(=O)CN(C)c2cc(Br)ccc2C(=O)O)c1</smiles>

1s. $431.6 \mathrm{mg}, 89 \%$, white solid (starting from $500.1 \mathrm{mg}$ of III-s); MP $297-298^{\circ} \mathrm{C} ;{ }^{1} \mathrm{H} \mathrm{NMR}\left(\mathrm{CDCl}_{3}\right.$, $400 \mathrm{MHz}): \delta$ 7.85-6.78 (m, 12H), $5.35(\mathrm{~s}, 1 \mathrm{H}), 5.17(\mathrm{~s}, 1 \mathrm{H}), 4.81(\mathrm{~s}, 2 \mathrm{H}), 2.42(\mathrm{~s}, 3 \mathrm{H}) ;{ }^{13} \mathrm{C} \mathrm{NMR}$ $\left(\mathrm{CDCl}_{3}, 100 \mathrm{MHz}\right): \delta 169.5,144.1,142.7,139.1,138.3,135.5,135.4,133.1,131.7,129.6,128.5$, 128.3, 127.9, 126.8, 126.6, 118.6, 55.0, 21.6; HRMS (TOF) m/z: $[\mathrm{M}-\mathrm{H}]^{-}$Calcd for $\mathrm{C}_{23} \mathrm{H}_{19} \mathrm{BrNO}_{4} \mathrm{~S}$ 484.5371; found 484.5376 .<smiles>C=C(CCN(C)c1ccccc1C(=O)OC)c1ccccc1</smiles>

III-t<smiles>C=C(CCN(C)c1ccccc1C(=O)O)c1ccccc1</smiles>

$5 a$

5a. $361.2 \mathrm{mg}, 86 \%$, colorless oil (starting from $435.5 \mathrm{mg}$ of III-t); ${ }^{1} \mathrm{H}$ NMR $\left(\mathrm{CDCl}_{3}, 400 \mathrm{MHz}\right)$ : $\delta$ 8.06-6.99 (m, 13H), $5.36(\mathrm{~s}, 1 \mathrm{H}), 5.09(\mathrm{~s}, 1 \mathrm{H}), 3.88(\mathrm{~d}, J=60.0 \mathrm{~Hz}, 2 \mathrm{H}), 2.93(\mathrm{~d}, J=36.0 \mathrm{~Hz}, 2 \mathrm{H})$, $2.38(\mathrm{~s}, 3 \mathrm{H}) ;{ }^{13} \mathrm{C} \mathrm{NMR}\left(\mathrm{CDCl}_{3}, 100 \mathrm{MHz}\right): \delta 169.8,144.6,143.9,139.9,138.4,135.3,132.9,132.2$, 131.9, 130.4, 129.6,128.7, 128.4, 127.9, 125.9,114.4, 51.3, 34.7, 21.5; HRMS (TOF) m/z: [M-H] Calcd for $\mathrm{C}_{24} \mathrm{H}_{22} \mathrm{NO}_{4} \mathrm{~S} 420.2965$; found 420.2966 .<smiles>C=C(COC(=O)c1ccccc1OCC(=C)c1ccccc1)c1ccccc1</smiles>

III-u 5b

5b. $221.0 \mathrm{mg}$, 87\%, yellow oil (starting from $268.2 \mathrm{mg}$ of III-u); ${ }^{1} \mathrm{H} \mathrm{NMR}\left(\mathrm{CDCl}_{3}, 400 \mathrm{MHz}\right)$ : $\delta$ $10.32(\mathrm{~s}$, broad, $1 \mathrm{H}), 8.16-7.11(\mathrm{~m}, 9 \mathrm{H}), 5.68(\mathrm{~s}, 1 \mathrm{H}), 5.54(\mathrm{~s}, 1 \mathrm{H}), 5.15(\mathrm{~s}, 2 \mathrm{H}) ;{ }^{13} \mathrm{C} \mathrm{NMR}\left(\mathrm{CDCl}_{3}\right.$, $100 \mathrm{MHz}): \delta 166.2,157.4,141.9,137.3,135.0,133.7,128.9,128.6,126.1,122.2,118.1,116.9$, 113.2, 71.7; HRMS (TOF) m/z: [M-H] $]^{-}$Calcd for $\mathrm{C}_{16} \mathrm{H}_{13} \mathrm{O}_{3} 253.1922$; found 253.1924.

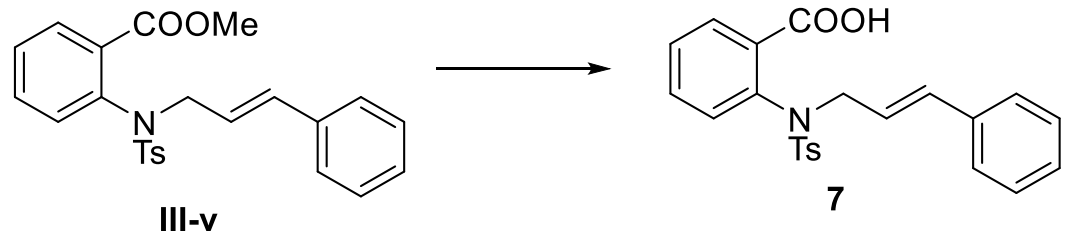


7. $341.9 \mathrm{mg}, 84 \%$, yellow solid (starting from $421.5 \mathrm{mg}$ of III-v); MP $244-245^{\circ} \mathrm{C}$; ${ }^{1} \mathrm{H}$ NMR $\left(\mathrm{CDCl}_{3}, 400 \mathrm{MHz}\right): \delta 8.02-7.01(\mathrm{~m}, 13 \mathrm{H}), 6.39-6.24(\mathrm{~m}, 2 \mathrm{H}), 4.48(\mathrm{~s}$, broad, $2 \mathrm{H}), 2.41(\mathrm{~s}, 3 \mathrm{H}) ;{ }^{13} \mathrm{C}$ $\mathrm{NMR}\left(\mathrm{CDCl}_{3}, 100 \mathrm{MHz}\right): \delta 169.9,143.9,138.2,136.3,135.9,134.5,132.9,132.1,131.7,131.2$, 129.6, 128.7, 128.6, 127.9, 126.5, 123.9, 54.5, 21.6; HRMS (TOF) $\mathrm{m} / \mathrm{z}:[\mathrm{M}-\mathrm{H}]^{-}$Calcd for $\mathrm{C}_{23} \mathrm{H}_{20} \mathrm{NO}_{4} \mathrm{~S} 406.8055$; found 406.8056 .

Asymmetric Bromolactonization in a $1 \mathrm{mmol}$ scale.<smiles>C=C(CN(c1ccccc1)c1ccccc1)c1ccccc1C(=O)O</smiles>

1a

$2 a$

To a solution of 1a (407.0 mg, $1.0 \mathrm{mmol}, 1.0$ equiv) and (DHQ) $)_{2} \mathrm{PHAL}$ (39.0 mg, $0.05 \mathrm{mmol}, 0.05$ equiv) in $\mathrm{PhMe}(40.0 \mathrm{~mL}$ ) was added NBS portionwise (212.4 mg, $1.2 \mathrm{mmol}, 1.2$ equiv) at room temperature. The mixture was then stirred for $6 \mathrm{~h}$ at room temperature. The reaction was quenched with saturated $\mathrm{Na}_{2} \mathrm{SO}_{3}(10.0 \mathrm{~mL})$ at room temperature. The solution was diluted with water $(30.0$ $\mathrm{mL}$ ) and extrated with EtOAc, dried over $\mathrm{MgSO}_{4}$ and concentrated in vacuo. The residue was purified by flash column chromatography (hexane/EtOAc) to yield the corresponding lactone 2a.

General Procedure for Asymmetric Halolactonization.

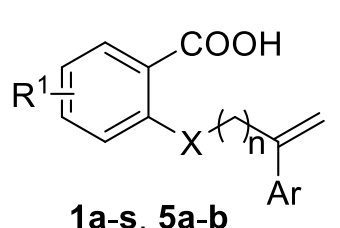
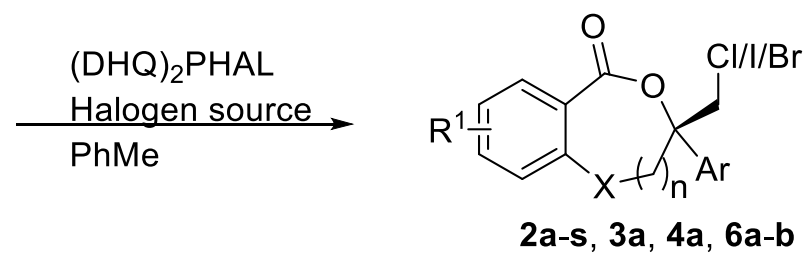

To a solution of 1 ( $0.1 \mathrm{mmol}, 1.0$ equiv) and (DHQ) $)_{2}$ PHAL (3.9 mg, $0.005 \mathrm{mmol}, 0.05$ equiv) in $\mathrm{PhMe}(4.0 \mathrm{~mL})$ was added halogen source $(0.12 \mathrm{mmol}, 1.2$ equiv) at room temperature. The mixture was then stirred for $5 \mathrm{~h}$ at room temperature. The reaction was quenched with saturated $\mathrm{Na}_{2} \mathrm{SO}_{3}(1.0$ $\mathrm{mL})$ at room temperature. The solution was diluted with water $(3.0 \mathrm{~mL})$ and extrated with EtOAc, dried over $\mathrm{MgSO}_{4}$ and concentrated in vacuo. The residue was purified by flash column chromatography (hexane/EtOAc) to yield the corresponding lactone $\mathbf{2 a - s , 3 a , ~} \mathbf{4 a}$ and $\mathbf{6 a - b}$.<smiles>CN1CC(CBr)(c2ccccc2)OC(=O)c2ccccc21</smiles>

(R)-3-(bromomethyl)-3-phenyl-1-tosyl-2,3-dihydrobenzo[e][1,4]oxazepin-5(1H)-one, $2 \mathrm{a}$ $47.0 \mathrm{mg}, 97 \%$; white solid (starting from $40.7 \mathrm{mg}$ of 1a); MP $170-171^{\circ} \mathrm{C}$; $[\alpha]_{D}^{25}=+9.5(c 1.0, \mathrm{MeOH}$, $99 \%$ ee); ${ }^{1} \mathrm{H} \mathrm{NMR}\left(\mathrm{CDCl}_{3}, 400 \mathrm{MHz}\right): \delta 7.52-6.97(\mathrm{~m}, 13 \mathrm{H}), 5.13\left(\mathrm{dd}, J_{1}=16.0 \mathrm{~Hz}, J_{2}=168.0 \mathrm{~Hz}\right.$,

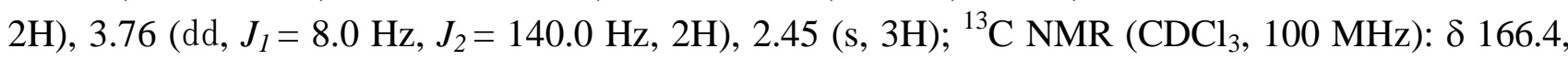
144.6, 138.3, 134.6, 134.5, 133.0, 132.3, 131.8, 130.7, 129.9, 128.8, 128.4, 127.7, 125.7, 82.2, 53.3, 40.2, 21.7; HRMS (TOF) $\mathrm{m} / \mathrm{z}$ : $[\mathrm{M}+\mathrm{H}]^{+} \mathrm{Calcd}$ for $\mathrm{C}_{23} \mathrm{H}_{21} \mathrm{BrNO}_{4} \mathrm{~S}$ 486.0347; found 486.0346; HPLC (Daicel Chiralcel OD-H, $i$-PrOH/Hexane $=35 / 65,1.0 \mathrm{~mL} / \mathrm{min}, 210 \mathrm{~nm}$ ) $\mathrm{t}_{1}=8.5 \mathrm{~min}\left(\right.$ minor), $\mathrm{t}_{2}=$ 11.9 min

(major). 


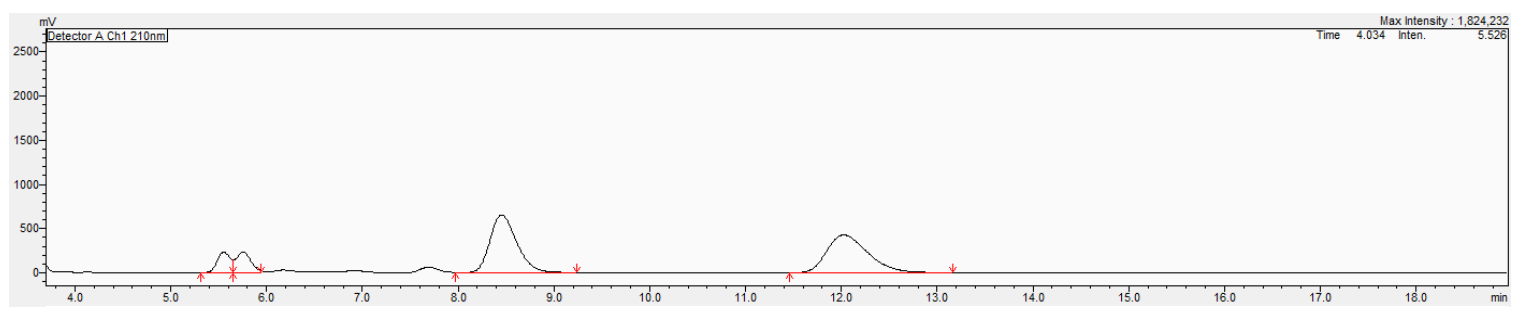

\begin{tabular}{|l|r|r|r|r|r|}
\hline Peak\# & Ret. Time & Area & Hei ght & Mark & \multicolumn{1}{|c|}{ Conc. } \\
\hline 1 & 5.553 & 2262050 & 235447 & M & 7.508 \\
\hline 2 & 5.755 & 2545329 & 236286 & M & 8.449 \\
\hline 3 & 8.454 & 12580449 & 651345 & M & 41.758 \\
\hline 4 & 12.025 & 12739162 & 427554 & M & 42.285 \\
\hline Total & & 30126990 & 1550632 & & 100.000 \\
\hline
\end{tabular}

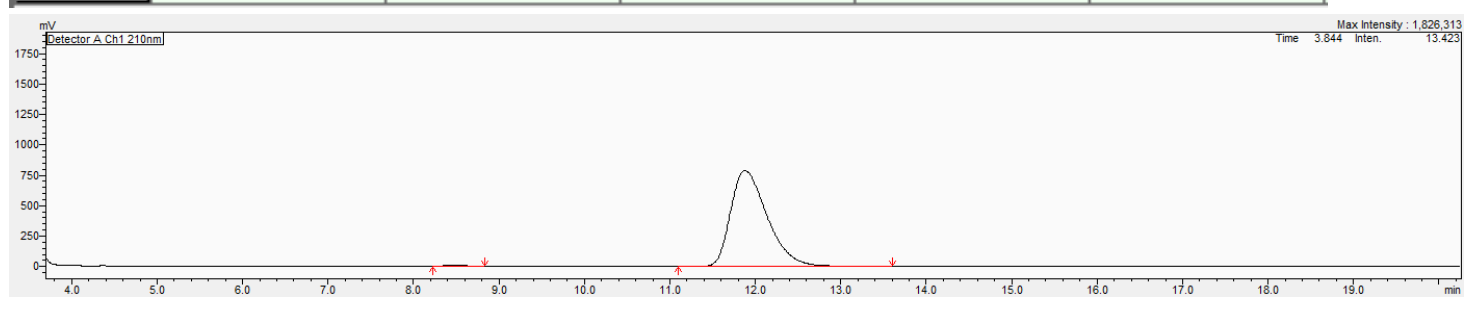

\begin{tabular}{|l|r|r|r|r|r|}
\hline Peak\# & Ret. Time & Area & Hei ght & Mark & \multicolumn{1}{c|}{ Conc. } \\
\hline 1 & 8.478 & 127254 & 7437 & $M$ & 0.540 \\
\hline 2 & 11.882 & 23441531 & 786387 & $M$ & 99.460 \\
\hline Total & & 23568785 & 793824 & & 100.000 \\
\hline
\end{tabular}<smiles>CN1C[C@@](CBr)(c2ccccc2)OC(=O)c2ccccc21</smiles>

(S)-3-(bromomethyl)-3-phenyl-1-tosyl-2,3-dihydrobenzo[e][1,4]oxazepin-5(1H)-one, ent-2a $47.5 \mathrm{mg}, 98 \%$; $[\alpha]_{D}^{25}=-9.7$ ( $c$ 1.0, $\mathrm{MeOH}, 94 \%$ ee); HPLC (Daicel Chiralcel OD-H, $i$-PrOH/Hexane $=35 / 65,1.0 \mathrm{~mL} / \mathrm{min}, 210 \mathrm{~nm}$ ) $\mathrm{t}_{1}=8.8 \mathrm{~min}$ (major), $\mathrm{t}_{2}=12.7 \mathrm{~min}$ (minor).

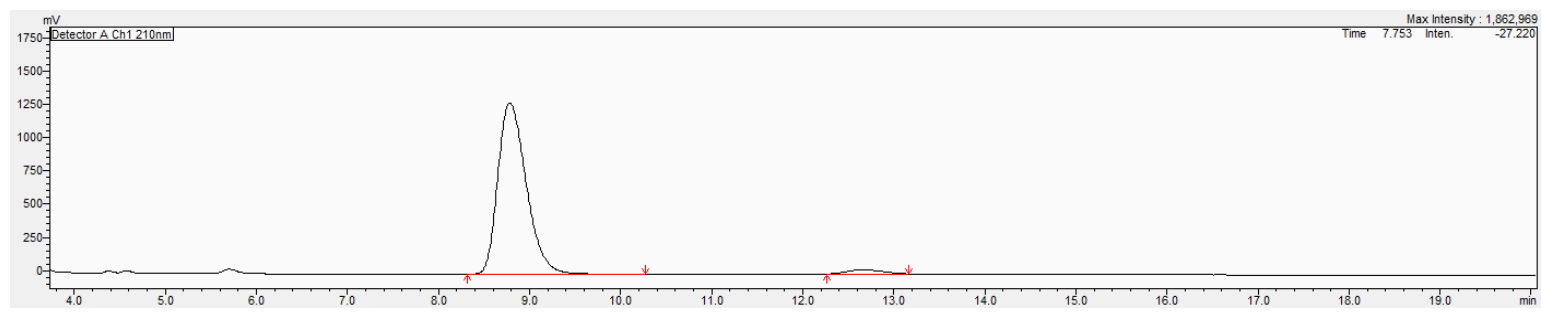

\begin{tabular}{|l|r|r|r|r|r|}
\hline Peak\# & Ret. Time & \multicolumn{1}{c|}{ Area } & \multicolumn{1}{c|}{ Hei ght } & \multicolumn{1}{c|}{ Mark } & \multicolumn{1}{c|}{ Conc. } \\
\hline 1 & 8.784 & 28035196 & 1292254 & V & 96.932 \\
\hline 2 & 12.669 & 887423 & 31726 & M & 3.068 \\
\hline Total & & 28922619 & 1323980 & & 100.000 \\
\hline
\end{tabular}<smiles>O=C1O[C@@H]2C(=O)O[C@@](CBr)(c3ccccc3)CN2c2ccccc21</smiles> 
(R)-3-(bromomethyl)-3-phenyl-1-(phenylsulfonyl)-2,3-dihydrobenzo[e][1,4]oxazepin-5(1H)-one, 2 b

$45.2 \mathrm{mg}$, 96\%; white solid (starting from $39.4 \mathrm{mg}$ of 1b); MP $168-169^{\circ} \mathrm{C}$; $[\alpha]_{D}^{25}=+10.7$ (c 1.0, $\mathrm{MeOH}$, $99 \%$ ee); ${ }^{1} \mathrm{H}$ NMR $\left(\mathrm{CDCl}_{3}, 400 \mathrm{MHz}\right): \delta 7.66-6.97(\mathrm{~m}, 14 \mathrm{H}), 5.15\left(\mathrm{dd}, J_{1}=16.0 \mathrm{~Hz}, J_{2}=172.0 \mathrm{~Hz}\right.$, $2 \mathrm{H}), 3.77\left(\mathrm{dd}, J_{l}=12.0 \mathrm{~Hz}, J_{2}=144.0 \mathrm{~Hz}, 2 \mathrm{H}\right) ;{ }^{13} \mathrm{C} \mathrm{NMR}\left(\mathrm{CDCl}_{3}, 100 \mathrm{MHz}\right): \delta 166.2,138.3,137.6$, 134.4, 133.6, 133.0, 132.3, 131.7, 130.8, 129.3, 128.9, 128.5, 127.6, 125.7, 82.2, 53.4, 40.2; HRMS (TOF) $\mathrm{m} / \mathrm{z}$ : $[\mathrm{M}+\mathrm{H}]^{+}$Calcd for $\mathrm{C}_{22} \mathrm{H}_{19} \mathrm{BrNO}_{4} \mathrm{~S}$ 472.0152; found 472.0154; HPLC (Daicel Chiralcel $\mathrm{OD}-\mathrm{H}, i$-PrOH$/ \mathrm{Hexane}=35 / 65,1.0 \mathrm{~mL} / \mathrm{min}, 210 \mathrm{~nm}$ ) $\mathrm{t}_{1}=10.4 \mathrm{~min}$ (minor), $\mathrm{t}_{2}=13.9 \mathrm{~min}$ (major).

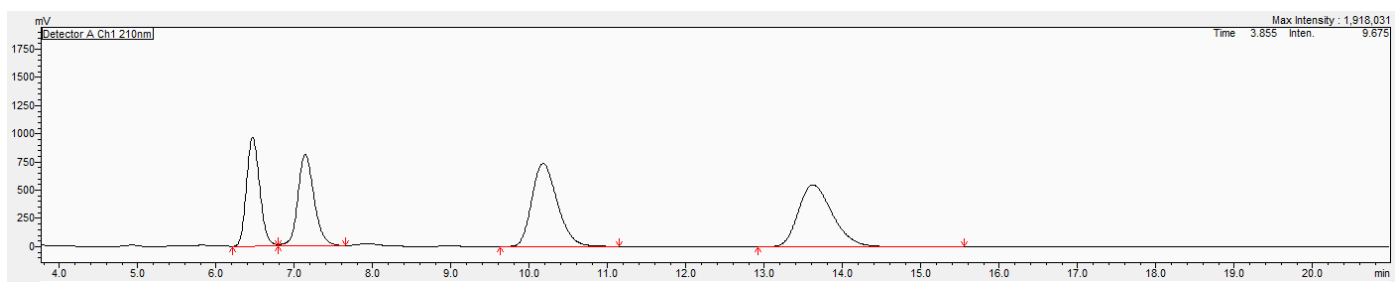

\begin{tabular}{|l|r|r|r|r|r|}
\hline Peak\# & Ret. Time & Area & Hei ght & Mark & Conc. \\
\hline 1 & 6.466 & 11262493 & 965366 & & 20.070 \\
\hline 2 & 7.137 & 11654804 & 810521 & V & 20.769 \\
\hline 3 & 10.175 & 16632614 & 733934 & M & 29.640 \\
\hline 4 & 13.621 & 16565250 & 549001 & S & 29.520 \\
\hline Total & & 56115160 & 3058822 & & 100.000 \\
\hline
\end{tabular}

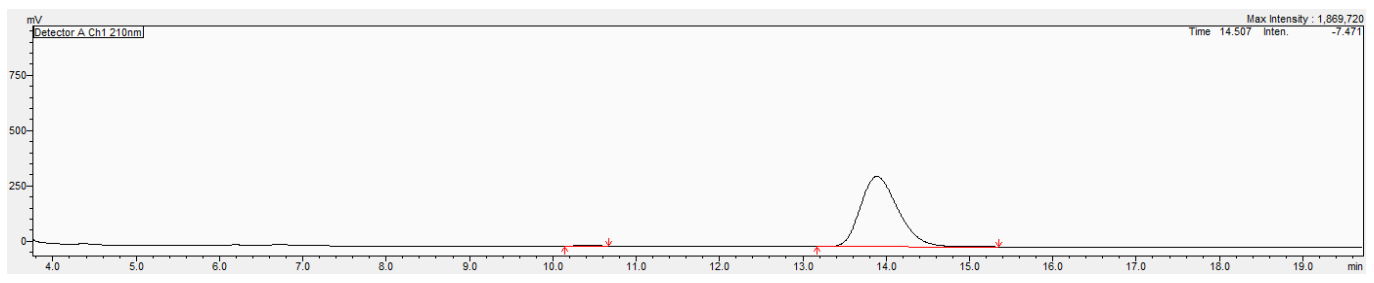

\begin{tabular}{|l|r|r|r|r|r|}
\hline Peak\# & Ret. Time & \multicolumn{1}{c|}{ Area } & Height & \multicolumn{1}{c|}{ Mark } & Conc. \\
\hline 1 & 10.412 & 65724 & 3673 & M & 0.657 \\
\hline 2 & 13.889 & 9936385 & 318337 & M & 99.343 \\
\hline Total & & 10002110 & 322010 & & 100.000 \\
\hline
\end{tabular}<smiles>COc1ccc(S(=O)(=O)N2C[C@](CBr)(c3ccccc3)OC(=O)c3ccccc32)cc1</smiles>

(R)-3-(bromomethyl)-1-((4-methoxyphenyl)sulfonyl)-3-phenyl-2,3-dihydrobenzo[e][1,4]oxazepin-5(1H)-one, $2 \mathrm{c}$

$47.6 \mathrm{mg}$, 95\%; yellow solid (starting from $42.3 \mathrm{mg}$ of $1 \mathrm{c})$; MP $199-200^{\circ} \mathrm{C}$; $[\alpha]_{D}^{25}=+8.1(c 1.0, \mathrm{MeOH}$, $99 \%$ ee); ${ }^{1} \mathrm{H}$ NMR $\left(\mathrm{CDCl}_{3}, 400 \mathrm{MHz}\right): \delta 7.57-6.92(\mathrm{~m}, 13 \mathrm{H}), 5.12\left(\mathrm{dd}, J_{l}=16.0 \mathrm{~Hz}, J_{2}=168.0 \mathrm{~Hz}\right.$,

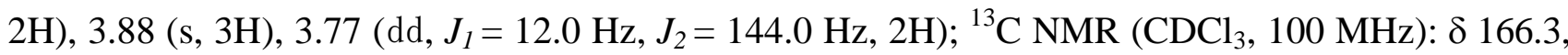
163.7, 138.4, 134.7, 133.0, 132.3, 131.8, 130.8, 129.8, 129.1, 128.8, 128.7, 128.4, 125.7, 114.5, 82.2, 55.7, 53.3, 40.3; HRMS (TOF) m/z: $[\mathrm{M}+\mathrm{H}]^{+}$Calcd for $\mathrm{C}_{23} \mathrm{H}_{21} \mathrm{BrNO}_{5} \mathrm{~S}$ 502.4497; found 502.4498; HPLC (Daicel Chiralcel OD-H, $i$-PrOH/Hexane $=35 / 65,1.0 \mathrm{~mL} / \mathrm{min}, 210 \mathrm{~nm}$ ) $\mathrm{t}_{1}=10.8 \mathrm{~min}$ (minor), $\mathrm{t}_{2}=14.8$ min (major). 


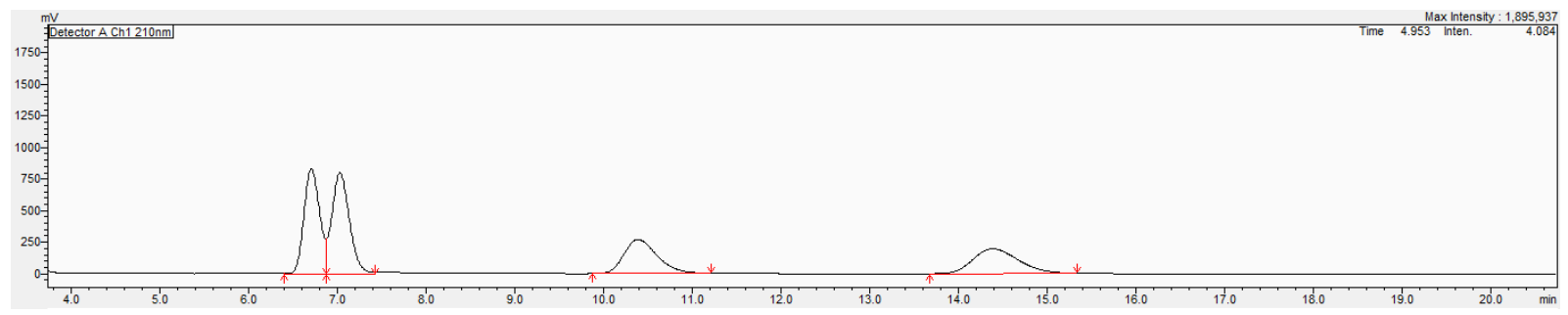

\begin{tabular}{|l|r|r|r|r|r|}
\hline Peak\# & Ret. Time & Area & Hei ght & Mark & \multicolumn{1}{c|}{ Conc. } \\
\hline 1 & 6.711 & 10220598 & 832562 & $V$ & 29.005 \\
\hline 2 & 7.033 & 11042356 & 801165 & $V$ & 31.337 \\
\hline 3 & 10.394 & 6995673 & 269007 & M & 19.853 \\
\hline 4 & 14.391 & 6978741 & 196480 & M & 19.805 \\
\hline Total & & 35237367 & 2099214 & & 100.000 \\
\hline
\end{tabular}

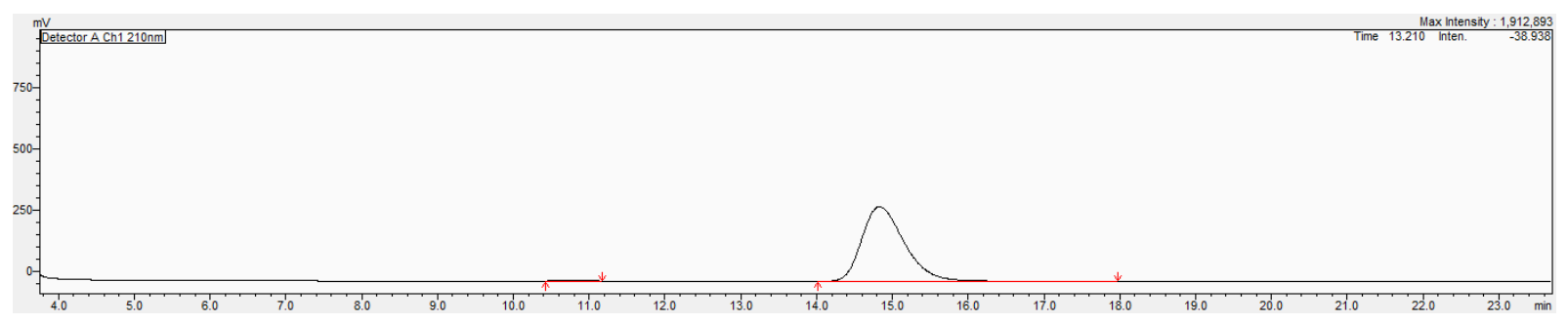

\begin{tabular}{|l|r|r|r|r|r|}
\hline Peak\# & Ret. Time & \multicolumn{1}{c|}{ Area } & Hei ght & \multicolumn{1}{c|}{ Mark } & \multicolumn{1}{c|}{ Conc. } \\
\hline 1 & 10.760 & 78573 & 3343 & M & 0.657 \\
\hline 2 & 14.831 & 11881859 & 304768 & $\mathrm{SV}$ & 99.343 \\
\hline Total & & 11960432 & 308111 & & 100.000 \\
\hline
\end{tabular}<smiles>O=C1OC(CBr)(c2ccccc2)CNc2ccccc21</smiles>

(R)-3-(bromomethyl)-1-((4-nitrophenyl)sulfonyl)-3-phenyl-2,3-dihydrobenzo[e][1,4]oxazepin-5(1H)-one, 2d $49.1 \mathrm{mg}$, 95\%; yellow solid (starting from $43.9 \mathrm{mg}$ of $1 \mathrm{~d}$ ); $\mathrm{MP} 228-229^{\circ} \mathrm{C} ;[\alpha]_{D}^{25}=+12.7(c 1.0$, $\mathrm{MeOH}, 98 \%$ ee); ${ }^{1} \mathrm{H} \mathrm{NMR}\left(\mathrm{CDCl}_{3}, 400 \mathrm{MHz}\right): \delta 8.33-6.95(\mathrm{~m}, 14 \mathrm{H}), 5.24\left(\mathrm{dd}, J_{l}=16.0 \mathrm{~Hz}, J_{2}=\right.$ $200.0 \mathrm{~Hz}, 2 \mathrm{H}), 3.80\left(\mathrm{dd}, J_{l}=12.0 \mathrm{~Hz}, J_{2}=160.0 \mathrm{~Hz}, 2 \mathrm{H}\right) ;{ }^{13} \mathrm{C} \mathrm{NMR}\left(\mathrm{CDCl}_{3}, 100 \mathrm{MHz}\right): \delta 166.1$, 150.6, 143.1, 138.0, 133.4, 133.3, 132.4, 131.7, 130.9, 129.6, 129.0, 128.7, 125.5, 124.5, 82.3, 53.3, 40.1; HRMS (TOF) $\mathrm{m} / \mathrm{z}$ : $[\mathrm{M}+\mathrm{H}]^{+}$Calcd for $\mathrm{C}_{22} \mathrm{H}_{18} \mathrm{BrN}_{2} \mathrm{O}_{6} \mathrm{~S}$ 517.9241; found 517.9242; HPLC (Daicel Chiralcel OD-H, $i$-PrOH/Hexane $=35 / 65,1.0 \mathrm{~mL} / \mathrm{min}, 210 \mathrm{~nm}$ ) $\mathrm{t}_{1}=17.1 \mathrm{~min}($ minor $), \mathrm{t}_{2}=$ 29.1 min (major). 


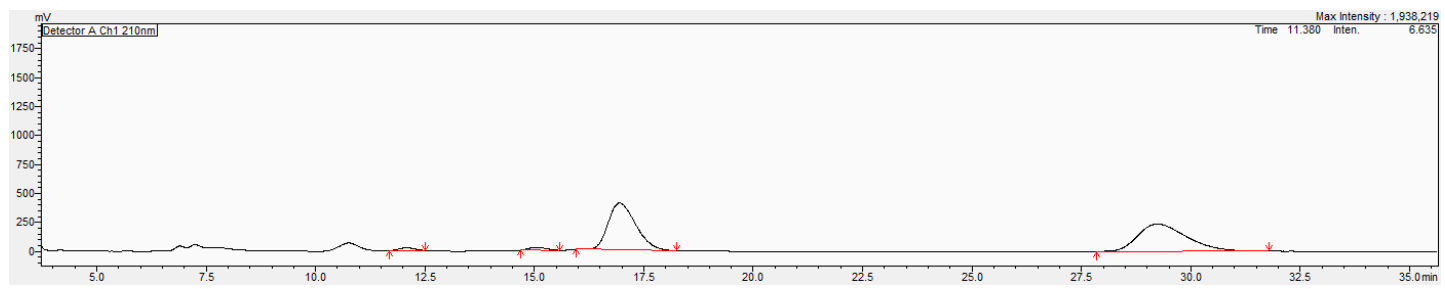

\begin{tabular}{|l|r|r|r|r|r|}
\hline Peak\# & Ret. Time & Area & Hei ght & \multicolumn{1}{c|}{ Mark } & \multicolumn{1}{c|}{ Conc. } \\
\hline 1 & 12.079 & 631689 & 24515 & M & 1.690 \\
\hline 2 & 15.050 & 666676 & 23319 & M & 1.783 \\
\hline 3 & 16.947 & 17819175 & 404809 & M & 47.665 \\
\hline 4 & 29.240 & 18266743 & 234896 & M & 48.862 \\
\hline Total & & 37384282 & 687539 & & 100.000 \\
\hline
\end{tabular}

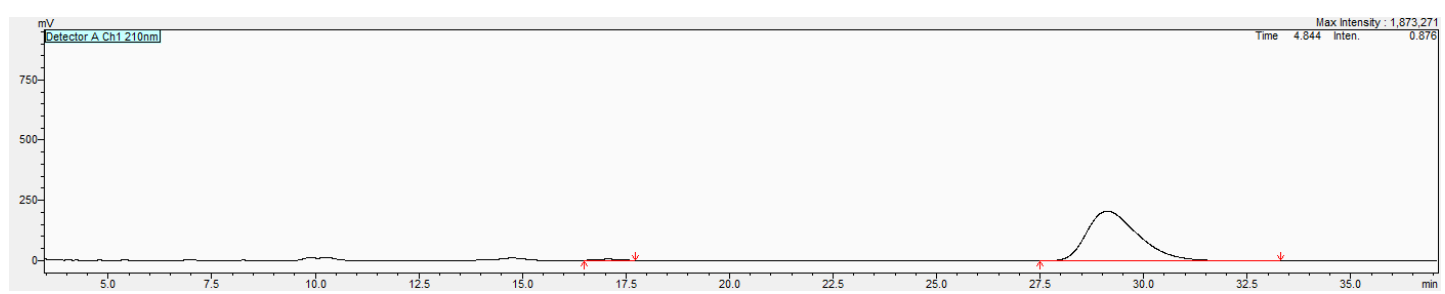

\begin{tabular}{|l|r|r|r|r|r|}
\hline Peak\# & Ret. Time & Area & \multicolumn{1}{c|}{ Hei ght } & \multicolumn{1}{|c|}{ Mark } & \multicolumn{1}{c|}{ Conc. } \\
\hline 1 & 17.099 & 218685 & 5361 & M & 1.207 \\
\hline 2 & 29.121 & 17903785 & 206466 & SV & 98.793 \\
\hline Total & & 18122470 & 211828 & & 100.000 \\
\hline
\end{tabular}<smiles>O=C1O[C@](CBr)(c2ccccc2)CNc2ccccc21</smiles>

(S)-3-(bromomethyl)-1-((4-nitrophenyl)sulfonyl)-3-phenyl-2,3-dihydrobenzo[e][1,4]oxazepin-5(1H)-one, ent-2d

$49.6 \mathrm{mg}, 96 \%$; $[\alpha]_{D}^{25}=-12.5$ (c 1.0, MeOH, 98\% ee); HPLC (Daicel Chiralcel OD-H, $i$-PrOH/Hexane $=35 / 65,1.0 \mathrm{~mL} / \mathrm{min}, 210 \mathrm{~nm}$ ) $\mathrm{t}_{1}=16.5 \mathrm{~min}$ (major), $\mathrm{t}_{2}=29.9 \min$ (minor).

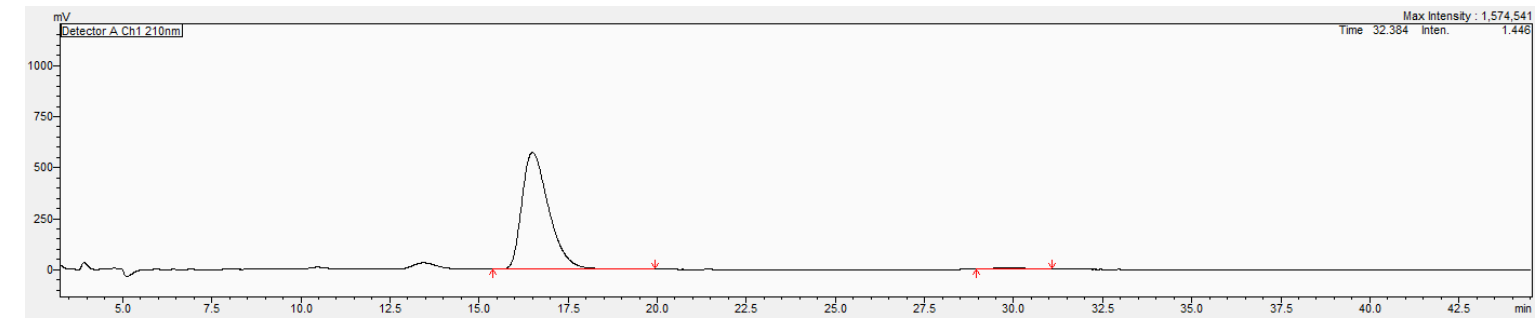

\begin{tabular}{|l|r|r|r|r|r|}
\hline Peak\# & Ret. Time & \multicolumn{1}{c|}{ Area } & \multicolumn{1}{c|}{ Mei ght } & \multicolumn{1}{c|}{ Mark } & \multicolumn{1}{c|}{ Conc. } \\
\hline 1 & 16.500 & 30032734 & 573147 & M & 98.798 \\
\hline 2 & 29.873 & 365508 & 5131 & M & 1.202 \\
\hline Total & & 30398242 & 578278 & & 100.000 \\
\hline
\end{tabular}<smiles>O=C1OC(CBr)(c2ccccc2)CNc2ccccc21</smiles> 
(R)-3-(bromomethyl)-1-(methylsulfonyl)-3-phenyl-2,3-dihydrobenzo[e][1,4]oxazepin-5(1H)-one, $2 \mathrm{e}$

$39.3 \mathrm{mg}, 96 \%$; yellow oil (starting from $33.2 \mathrm{mg}$ of $1 \mathrm{e})$; $[\alpha]_{D}^{25}=+11.6\left(c 1.0, \mathrm{MeOH}, 95 \%\right.$ ee); ${ }^{1} \mathrm{H}$ NMR $\left(\mathrm{CDCl}_{3}, 400 \mathrm{MHz}\right): \delta$ 7.44-7.09 (m, 9H), $5.06\left(\mathrm{dd}, J_{l}=16.0 \mathrm{~Hz}, J_{2}=148.0 \mathrm{~Hz}, 2 \mathrm{H}\right), 3.93(\mathrm{dd}$, $\left.J_{l}=12.0 \mathrm{~Hz}, J_{2}=176.0 \mathrm{~Hz}, 2 \mathrm{H}\right), 3.02(\mathrm{~s}, 3 \mathrm{H}) ;{ }^{13} \mathrm{C} \mathrm{NMR}\left(\mathrm{CDCl}_{3}, 100 \mathrm{MHz}\right): \delta 166.4,144.6,138.3$, $134.6,134.5,133.0,132.3,131.8,130.7,129.9,128.8,128.4,127.7,125.7,82.2,53.3,40.2$, 21.7167.6, 138.3 134.3, 133.5, 132.2, 131.4, 130.8, 129.3, 128.9, 128.7, 125.7, 82.6,53.2, 40.3, 38.7; HRMS (TOF) m/z: $[\mathrm{M}+\mathrm{H}]^{+}$Calcd for $\mathrm{C}_{17} \mathrm{H}_{17} \mathrm{BrNO}_{4} \mathrm{~S}$ 410.3850; found 410.384; HPLC (Daicel Chiralcel OD-H, $i$-PrOH/Hexane $=35 / 65,1.0 \mathrm{~mL} / \mathrm{min}, 210 \mathrm{~nm}$ ) $\mathrm{t}_{1}=13.9 \mathrm{~min}$ (minor), $\mathrm{t}_{2}=16.9 \mathrm{~min}$ (major).

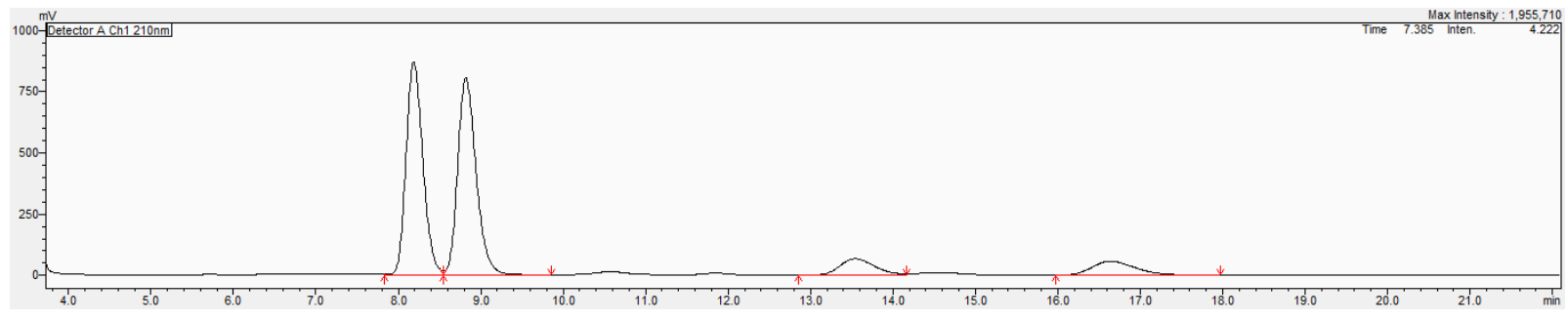

\begin{tabular}{|l|r|r|r|r|r|}
\hline Peak\# & Ret. Time & \multicolumn{1}{c|}{ Area } & \multicolumn{1}{c|}{ Hei ght } & \multicolumn{1}{c|}{ Mark } & \multicolumn{1}{c|}{ Conc. } \\
\hline 1 & 8.186 & 12353105 & 869227 & V & 42.486 \\
\hline 2 & 8.820 & 12604543 & 805905 & V & 43.351 \\
\hline 3 & 13.544 & 2007243 & 67370 & V & 6.904 \\
\hline 4 & 16.642 & 2110828 & 57116 & M & 7.260 \\
\hline Total & & 29075720 & 1799618 & & 100.000 \\
\hline
\end{tabular}

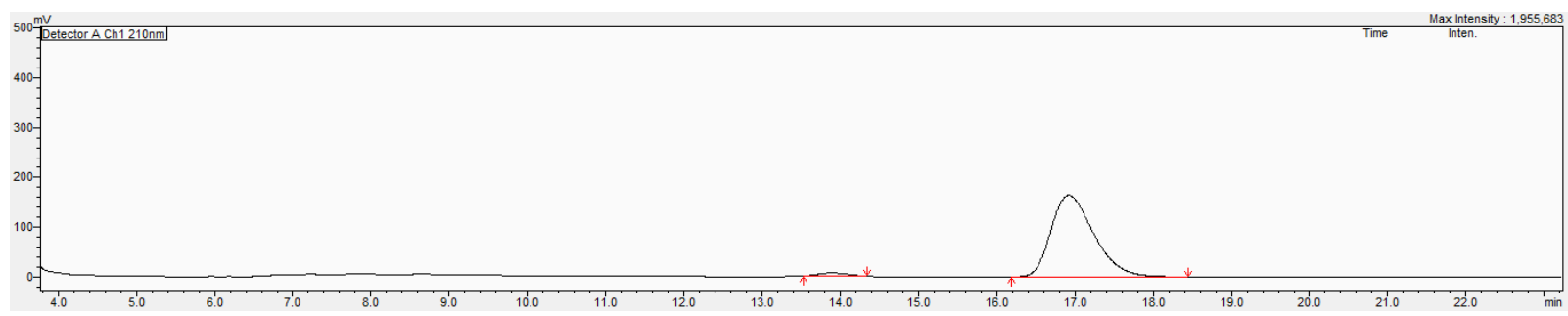

\begin{tabular}{|l|r|r|r|r|r|}
\hline Peak\# & Ret. Time & \multicolumn{1}{c|}{ Mrea } & Hei ght & \multicolumn{1}{c|}{ Mark } & \multicolumn{1}{c|}{ Conc. } \\
\hline 1 & 13.887 & 163627 & 6353 & M & 2.542 \\
\hline 2 & 16.920 & 6272471 & 164407 & $V$ & 97.458 \\
\hline Total & & 6436098 & 170759 & & 100.000 \\
\hline
\end{tabular}<smiles>Cc1ccccc1C1(CBr)CN(C)c2ccccc2C(=O)O1</smiles>

\section{(R)-3-(bromomethyl)-3-(o-tolyl)-1-tosyl-2,3-dihydrobenzo[e][1,4]oxazepin-5(1H)-one, $2 \mathrm{f}$}

$44.9 \mathrm{mg}, 90 \%$; white solid (starting from $42.1 \mathrm{mg}$ of 1f); $\mathrm{MP} 193-194^{\circ} \mathrm{C} ;[\alpha]_{D}^{25}=+5.9(c 1.0, \mathrm{MeOH}$, $91 \%$ ee); ${ }^{1} \mathrm{H} \mathrm{NMR}\left(\mathrm{CDCl}_{3}, 400 \mathrm{MHz}\right): \delta 7.53-6.79(\mathrm{~m}, 12 \mathrm{H}), 5.04\left(\mathrm{dd}, J_{l}=12.0 \mathrm{~Hz}, J_{2}=92.0 \mathrm{~Hz}\right.$, $2 \mathrm{H}), 3.90\left(\mathrm{dd}, J_{1}=12.0 \mathrm{~Hz}, J_{2}=172.0 \mathrm{~Hz}, 2 \mathrm{H}\right), 2.45(\mathrm{~s}, 3 \mathrm{H}), 2.36(\mathrm{~s}, 3 \mathrm{H}) ;{ }^{13} \mathrm{C} \mathrm{NMR}\left(\mathrm{CDCl}_{3}, 100\right.$ $\mathrm{MHz}): \delta 166.0,144.6,135.0,134.9,134.7,134.2,133.4,132.8,131.9,131.4,129.9,128.6,128.3$, 127.9, 127.6, 125.8, 83.3, 55.0, 36.8,21.7,21.2; HRMS (TOF) $\mathrm{m} / \mathrm{z}:[\mathrm{M}+\mathrm{H}]^{+}$Calcd for $\mathrm{C}_{24} \mathrm{H}_{23} \mathrm{BrNO}_{4} \mathrm{~S}$ 
500.6148; found 500.6149; HPLC (Daicel Chiralcel OD-H, $i-\mathrm{PrOH} / \mathrm{Hexane}=35 / 65,1.0 \mathrm{~mL} / \mathrm{min}$, $210 \mathrm{~nm}) \mathrm{t}_{1}=10.9 \min$ (major), $\mathrm{t}_{2}=18.2 \min$ (minor).

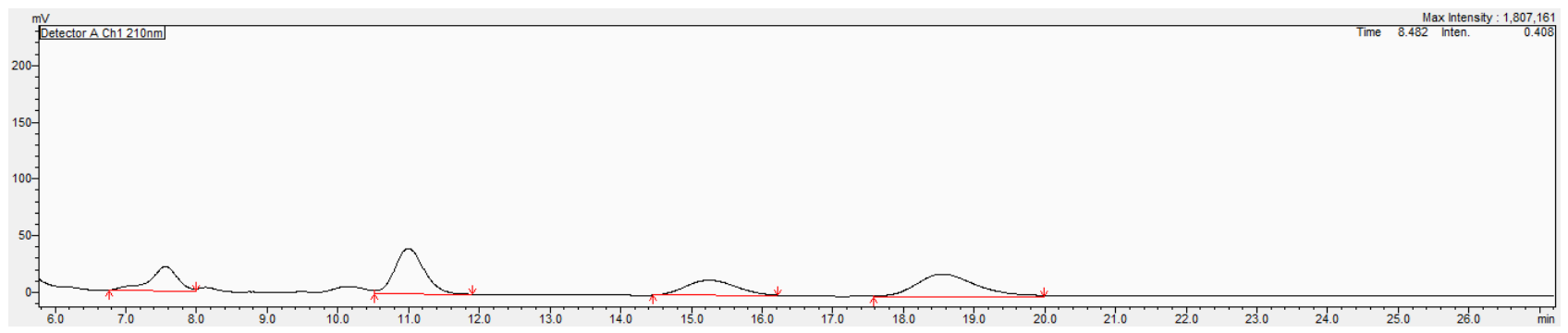

\begin{tabular}{|l|r|r|r|r|r|}
\hline Peak\# & Ret. Time & Area & Hei ght & Mark & \multicolumn{1}{c|}{ Conc. } \\
\hline 1 & 7.559 & 604030 & 21510 & $\mathrm{M}$ & 16.877 \\
\hline 2 & 10.995 & 1160074 & 39974 & $\mathrm{~V}$ & 32.413 \\
\hline 3 & 15.264 & 653578 & 13272 & $\mathrm{M}$ & 18.262 \\
\hline 4 & 18.542 & 1161304 & 19750 & $\mathrm{~V}$ & 32.448 \\
\hline Total & & 3578986 & 94506 & & 100.000 \\
\hline
\end{tabular}

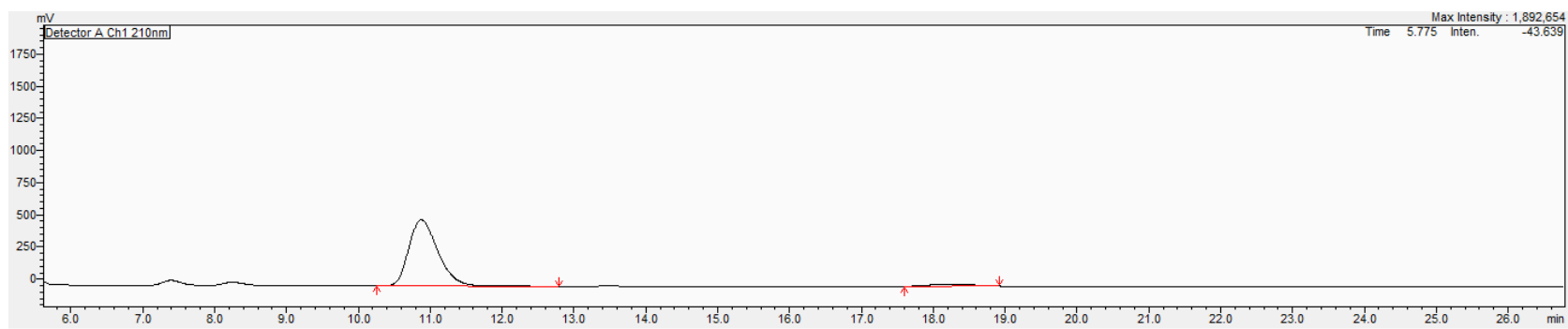

\begin{tabular}{|l|r|r|r|r|r|}
\multicolumn{1}{|c|}{ Peak\# } & Ret. Time & \multicolumn{1}{c|}{ Area } & \multicolumn{1}{c|}{ Hei ght } & \multicolumn{1}{c|}{ Mark } & \multicolumn{1}{c|}{ Conc. } \\
\hline 1 & 10.874 & 14548517 & 515199 & & 95.449 \\
\hline 2 & 18.244 & 693677 & 15980 & M & 4.551 \\
\hline Total & & 15242194 & 531179 & & 100.000 \\
\hline
\end{tabular}<smiles>Cc1cccc(C2(CBr)CN(C)c3ccccc3C(=O)O2)c1</smiles>

(R)-3-(bromomethyl)-3-(m-tolyl)-1-tosyl-2,3-dihydrobenzo[e][1,4]oxazepin-5(1H)-one, $2 \mathrm{~g}$

$47.4 \mathrm{mg}, 95 \%$; white solid (starting from $42.2 \mathrm{mg}$ of $1 \mathrm{~g}$ ); $\mathrm{MP} 191-192^{\circ} \mathrm{C} ;[\alpha]_{D}^{25}=+15.4(c 1.0, \mathrm{MeOH}$, 99.5\% ee); ${ }^{1} \mathrm{H} \mathrm{NMR}\left(\mathrm{CDCl}_{3}, 400 \mathrm{MHz}\right): \delta 7.53-6.82(\mathrm{~m}, 12 \mathrm{H}), 5.11\left(\mathrm{dd}, J_{1}=16.0 \mathrm{~Hz}, J_{2}=172.0 \mathrm{~Hz}\right.$, $2 \mathrm{H}), 3.77\left(\mathrm{dd}, J_{l}=12.0 \mathrm{~Hz}, J_{2}=148.0 \mathrm{~Hz}, 2 \mathrm{H}\right), 2.45(\mathrm{~s}, 3 \mathrm{H}), 2.14(\mathrm{~s}, 3 \mathrm{H}) ;{ }^{13} \mathrm{C} \mathrm{NMR}\left(\mathrm{CDCl}_{3}, 100\right.$ $\mathrm{MHz}): \delta 166.4,144.5,138.5,138.2,134.6,132.7,132.3,131.7,130.6,129.9,129.1,128.7,127.7$, 126.5, 122.8, 82.2, 53.4, 40.2, 21.7, 21.2; HRMS (TOF) m/z: $[\mathrm{M}+\mathrm{H}]^{+}$Calcd for $\mathrm{C}_{24} \mathrm{H}_{23} \mathrm{BrNO}_{4} \mathrm{~S}$ 500.6148; found 500.6147; HPLC (Daicel Chiralcel OD-H, $i$-PrOH/Hexane $=35 / 65,1.0 \mathrm{~mL} / \mathrm{min}$, $210 \mathrm{~nm}$ ) $\mathrm{t}_{1}=9.5 \min$ (major), $\mathrm{t}_{2}=20.8 \mathrm{~min}$ (minor). 


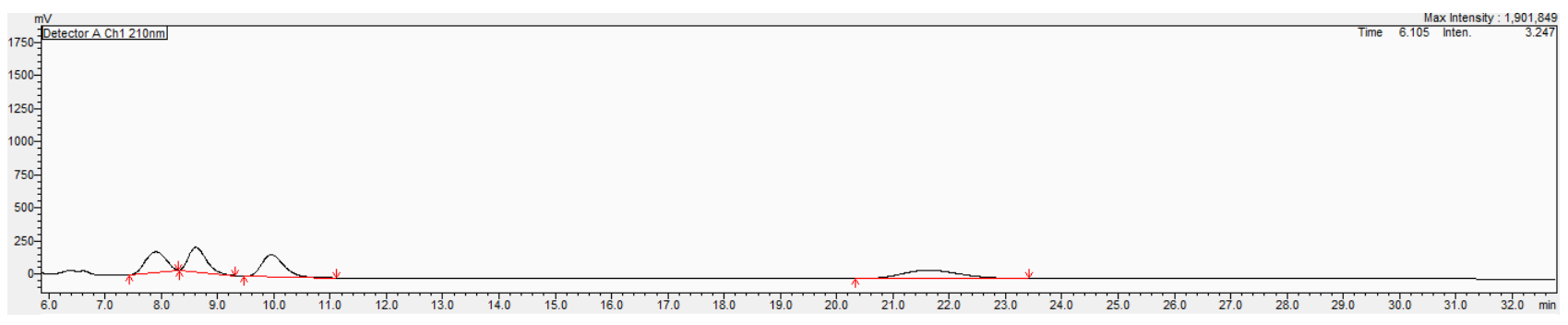

\begin{tabular}{|l|r|r|r|r|r|}
\hline Peak\# & Ret. Time & \multicolumn{1}{c|}{ Area } & \multicolumn{1}{c|}{ Hei ght } & \multicolumn{1}{c|}{ Mark } & \multicolumn{1}{c|}{ Conc. } \\
\hline 1 & 7.905 & 3902577 & 154595 & & 22.962 \\
\hline 2 & 8.613 & 3991333 & 184678 & M & 23.485 \\
\hline 3 & 9.953 & 4545350 & 166155 & & 26.744 \\
\hline 4 & 21.634 & 4556221 & 63348 & M & 26.808 \\
\hline Total & & 16995481 & 568776 & & 100.000 \\
\hline
\end{tabular}

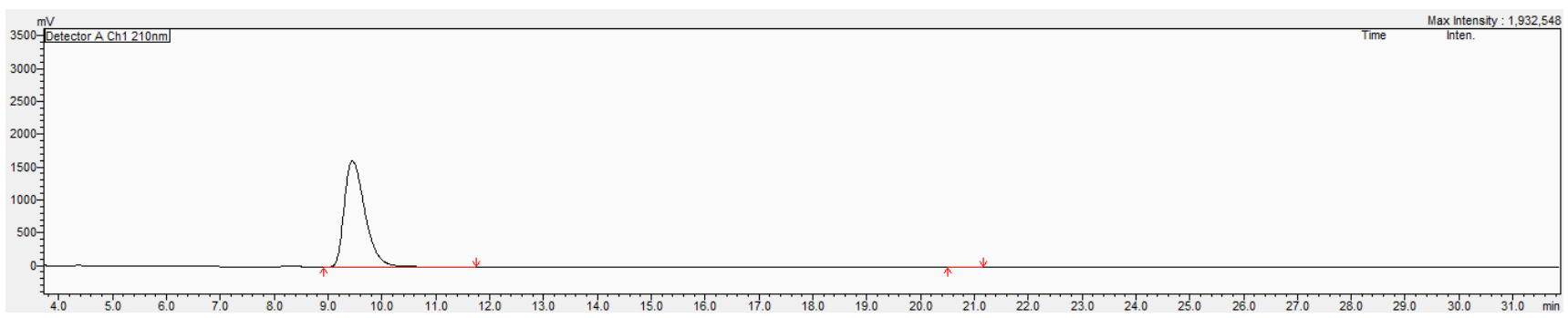

\begin{tabular}{|l|r|r|r|r|r|}
\hline Peak\# & Ret. Time & \multicolumn{1}{c|}{ Area } & \multicolumn{1}{c|}{ Hei ght } & \multicolumn{1}{|c|}{ Mark } & Conc. \\
\hline 1 & 9.452 & 43249279 & 1609909 & & 99.992 \\
\hline 2 & 20.766 & 3611 & 147 & M & 0.008 \\
\hline Total & & 43252889 & 1610055 & & 100.000 \\
\hline
\end{tabular}<smiles>Cc1ccc(C2(CBr)CN(C)c3ccccc3C(=O)O2)cc1</smiles>

(R)-3-(bromomethyl)-3-(p-tolyl)-1-tosyl-2,3-dihydrobenzo[e][1,4]oxazepin-5(1H)-one, $2 \mathrm{~h}$

$48.4 \mathrm{mg}$, 97\%; white solid (starting from $42.0 \mathrm{mg}$ of $\mathbf{1 h}$ ); $\mathrm{MP} 198-199^{\circ} \mathrm{C}$; $[\alpha]_{D}^{25}=+6.3(\mathrm{c} 1.0, \mathrm{MeOH}$, 99.5\% ee); ${ }^{1} \mathrm{H}$ NMR $\left(\mathrm{CDCl}_{3}, 400 \mathrm{MHz}\right): \delta 7.52-6.85(\mathrm{~m}, 12 \mathrm{H}), 5.12\left(\mathrm{dd}, J_{l}=16.0 \mathrm{~Hz}, J_{2}=180.0 \mathrm{~Hz}\right.$, $2 \mathrm{H}), 3.74\left(\mathrm{dd}, J_{l}=12.0 \mathrm{~Hz}, J_{2}=152.0 \mathrm{~Hz}, 2 \mathrm{H}\right), 2.44(\mathrm{~s}, 3 \mathrm{H}), 2.16(\mathrm{~s}, 3 \mathrm{H}) ;{ }^{13} \mathrm{C} \mathrm{NMR}\left(\mathrm{CDCl}_{3}, 100\right.$ $\mathrm{MHz}): \delta 166.4,144.5,138.4,135.3,134.7,134.5,132.9,132.3,131.7,130.7,129.8,129.4,128.7$, 127.7,125.6,82.2, 53.3, 40.4, 21.7, 20.8; HRMS (TOF) $\mathrm{m} / \mathrm{z}:[\mathrm{M}+\mathrm{H}]^{+}$Calcd for $\mathrm{C}_{24} \mathrm{H}_{23} \mathrm{BrNO}_{4} \mathrm{~S}$ 500.6148; found 500.6147; HPLC (Daicel Chiralcel OD-H, $i-\mathrm{PrOH} / \mathrm{Hexane}=35 / 65,1.0 \mathrm{~mL} / \mathrm{min}$, $210 \mathrm{~nm}$ ) $\mathrm{t}_{1}=12.8 \mathrm{~min}$ (minor), $\mathrm{t}_{2}=13.9$ min (major). 


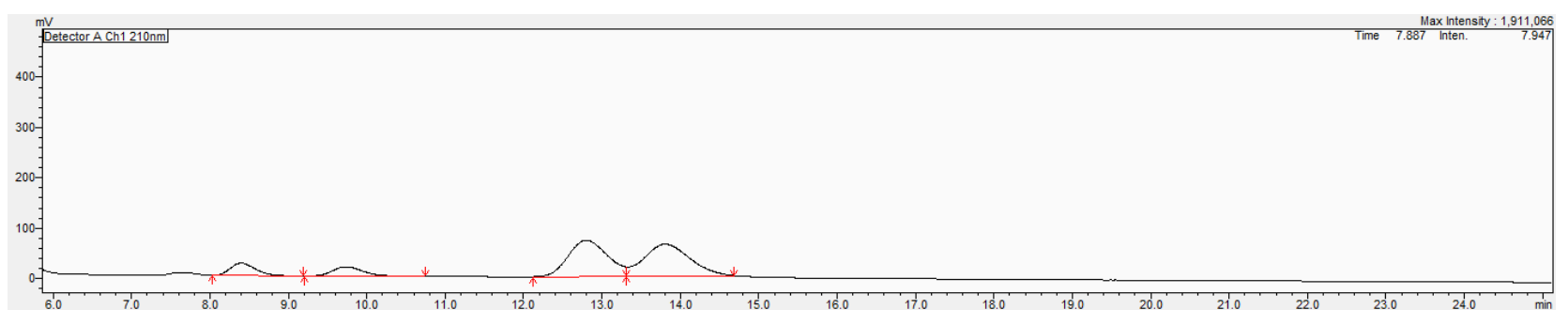

\begin{tabular}{|l|r|r|r|r|r|}
\hline Peak\# & Ret. Time & Area & Hei ght & Mark & \multicolumn{1}{c|}{ Conc. } \\
\hline 1 & 8.397 & 551413 & 24619 & & 8.856 \\
\hline 2 & 9.732 & 518647 & 18773 & & 8.330 \\
\hline 3 & 12.802 & 2541951 & 72001 & M & 40.826 \\
\hline 4 & 13.803 & 2614281 & 63497 & M & 41.988 \\
\hline Total & & 6226293 & 178891 & & 100.000 \\
\hline
\end{tabular}

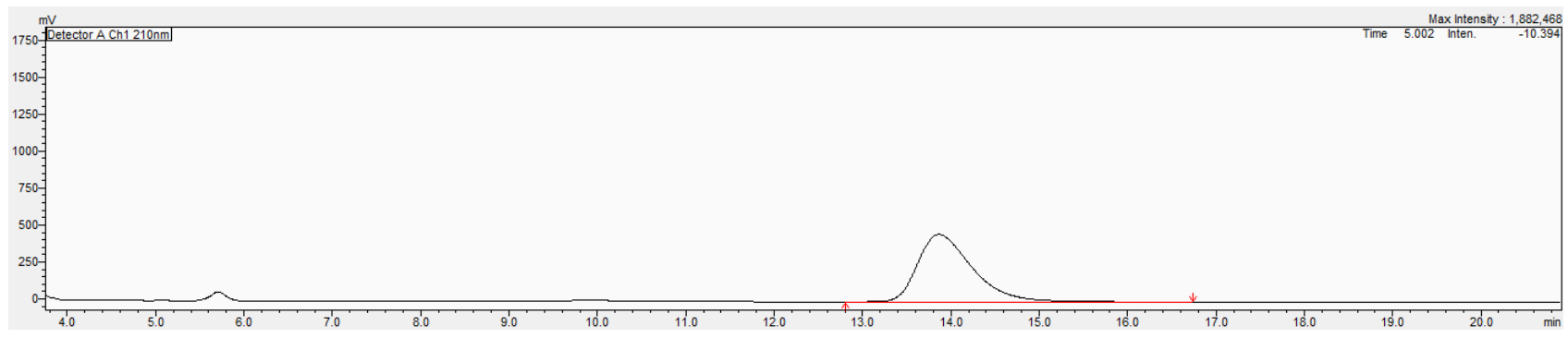

\begin{tabular}{|c|c|c|r|r|r|}
\hline Peak\# & Ret. Time & Area & \multicolumn{1}{c|}{ Hei ght } & Mark & \multicolumn{1}{c|}{ Conc. } \\
\hline 1 & 13.865 & 19627503 & 455880 & SV & 100.000 \\
\hline Total & & 19627503 & 455880 & & 100.000 \\
\hline
\end{tabular}<smiles>COc1cccc([C@@]2(CBr)CN([13CH3])c3ccccc3C(=O)O2)c1</smiles>

(R)-3-(bromomethyl)-3-(3-methoxyphenyl)-1-tosyl-2,3-dihydrobenzo[e][1,4]oxazepin-5(1H)-one, $2 \mathrm{i}$ $51.0 \mathrm{mg}$, 99\%; yellow solid (starting from $43.7 \mathrm{mg}$ of $1 \mathrm{i}$ ); $\mathrm{MP} 202-203^{\circ} \mathrm{C} ;[\alpha]_{D}^{25}=+7.4(c 1.0, \mathrm{MeOH}$, $98 \%$ ee); ${ }^{1} \mathrm{H} \mathrm{NMR}\left(\mathrm{CDCl}_{3}, 400 \mathrm{MHz}\right): \delta 7.52-6.55(\mathrm{~m}, 12 \mathrm{H}), 5.13\left(\mathrm{dd}, J_{l}=12.0 \mathrm{~Hz}, J_{2}=188.0 \mathrm{~Hz}\right.$, $2 \mathrm{H}), 3.76\left(\mathrm{dd}, J_{1}=8.0 \mathrm{~Hz}, J_{2}=140.0 \mathrm{~Hz}, 2 \mathrm{H}\right), 3.64(\mathrm{~s}, 3 \mathrm{H}), 2.44(\mathrm{~s}, 3 \mathrm{H}) ;{ }^{13} \mathrm{C} \mathrm{NMR}\left(\mathrm{CDCl}_{3}, 100\right.$ $\mathrm{MHz}): \delta 166.4,159.6,144.6,139.9,134.7,134.6,133.0,132.2,131.8,130.8,129.9,128.8,127.7$, 118.1, 114.0, 111.6, 82.1, 55.3, 53.5, 40.1, 21.7; HRMS (TOF) m/z: $[\mathrm{M}+\mathrm{H}]^{+}$Calcd for $\mathrm{C}_{24} \mathrm{H}_{23} \mathrm{BrNO}_{5} \mathrm{~S}$ 516.7044; found 516.7045; HPLC (Daicel Chiralcel OD-H, $i$-PrOH/Hexane = 35/65, $1.0 \mathrm{~mL} / \mathrm{min}, 210 \mathrm{~nm}$ ) $\mathrm{t}_{1}=9.6 \min$ (minor), $\mathrm{t}_{2}=16.4 \min$ (major). 


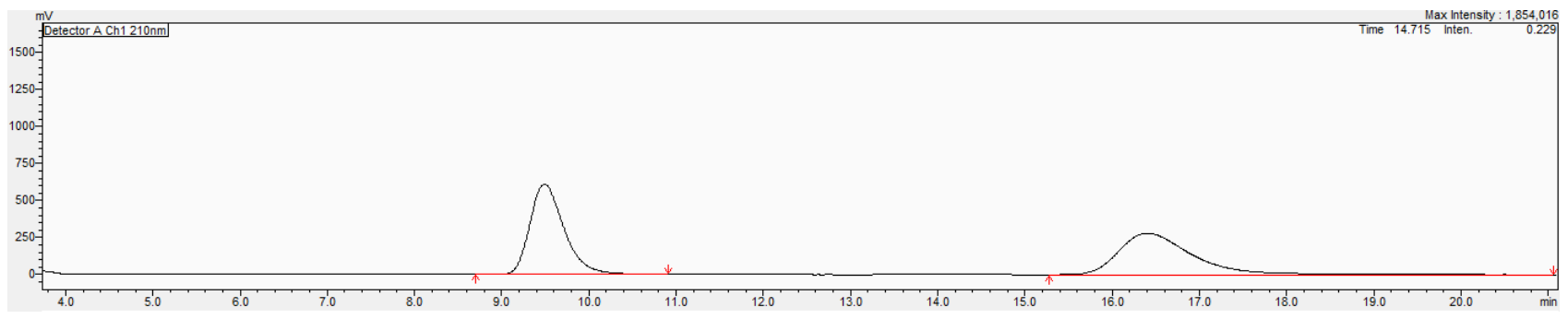

\begin{tabular}{|l|r|r|r|r|r|}
\hline Peak\# & Ret. Time & Area & Hei ght & Mark & \multicolumn{1}{c|}{ Conc. } \\
\hline 1 & 9.491 & 16484260 & 608639 & M & 50.197 \\
\hline 2 & 16.406 & 16354621 & 279382 & $\mathrm{~S}$ & 49.803 \\
\hline Total & & 32838881 & 888022 & & 100.000 \\
\hline
\end{tabular}

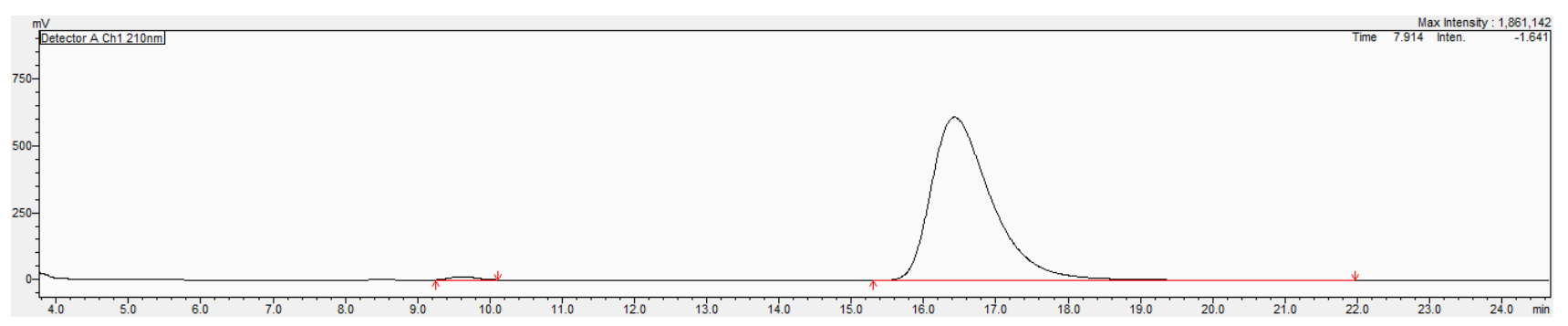

\begin{tabular}{|l|r|r|r|r|r|}
\hline Peak\# & Ret. Time & Area & Hei ght & \multicolumn{1}{c|}{ Mark } & \multicolumn{1}{c|}{ Conc. } \\
\hline 1 & 9.613 & 314011 & 12514 & M & 0.880 \\
\hline 2 & 16.429 & 35350097 & 609391 & $S V$ & 99.120 \\
\hline Total & & 35664108 & 621904 & & 100.000 \\
\hline
\end{tabular}<smiles>CS(=O)(=O)c1cccc(Br)c1</smiles>

(R)-3-(bromomethyl)-3-(3-bromophenyl)-1-tosyl-2,3-dihydrobenzo[e][1,4]oxazepin-5(1H)-one, $2 \mathbf{j}$

$53.8 \mathrm{mg}$, 95\%; yellow solid (starting from $48.5 \mathrm{mg}$ of $\mathbf{1 j}$ ); MP $209-210^{\circ} \mathrm{C} ;[\alpha]_{D}^{25}=+11.8(c 1.0$, $\mathrm{MeOH}, 97 \%$ ee); ${ }^{1} \mathrm{H} \mathrm{NMR}\left(\mathrm{CDCl}_{3}, 400 \mathrm{MHz}\right): \delta 7.52-6.95(\mathrm{~m}, 12 \mathrm{H}), 5.04\left(\mathrm{dd}, J_{1}=16.0 \mathrm{~Hz}, J_{2}=\right.$ $152.0 \mathrm{~Hz}, 2 \mathrm{H}), 3.72\left(\mathrm{dd}, J_{1}=8.0 \mathrm{~Hz}, J_{2}=124.0 \mathrm{~Hz}, 2 \mathrm{H}\right), 2.44(\mathrm{~s}, 3 \mathrm{H}) ;{ }^{13} \mathrm{C} \mathrm{NMR}\left(\mathrm{CDCl}_{3}, 100 \mathrm{MHz}\right)$ : $\delta 166.8,144.7,140.7,134.6,133.3,132.0,131.8,131.5,130.8,130.3,129.9,129.1,129.0,127.6$, 124.3, 122.8, 81.6, 53.7, 39.8, 21.7; HRMS (TOF) m/z: [M+H] ${ }^{+}$Calcd for $\mathrm{C}_{23} \mathrm{H}_{20} \mathrm{Br}_{2} \mathrm{NO}_{4} \mathrm{~S} 566.9903$; found 566.9904; HPLC (Daicel Chiralcel OD-H, $i-\mathrm{PrOH} / \mathrm{Hexane}=30 / 70,1.0 \mathrm{~mL} / \mathrm{min}, 210 \mathrm{~nm}$ ) $\mathrm{t}_{1}=$ $10.7 \min$ (minor), $\mathrm{t}_{2}=13.7 \mathrm{~min}$ (major).

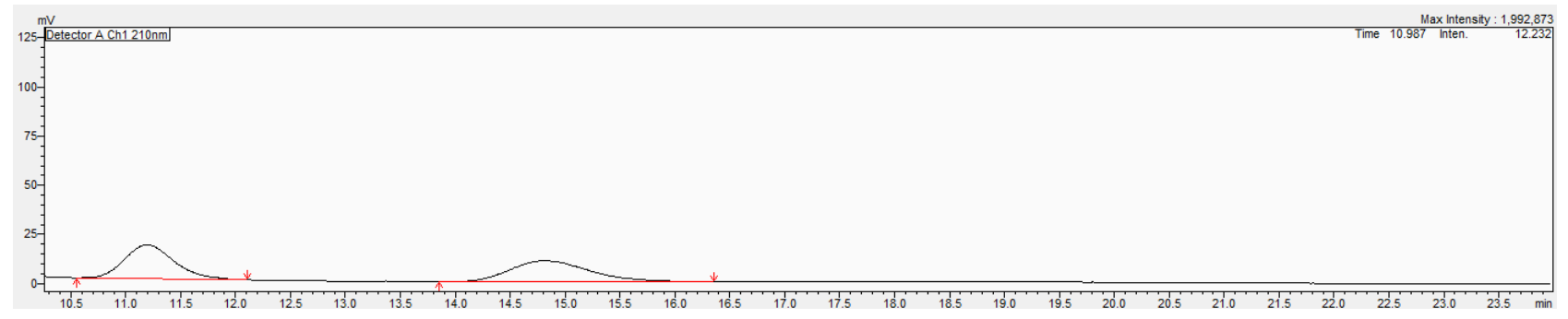

\begin{tabular}{|l|r|r|r|r|r|}
\hline Peak\# & Ret. Time & Area & Hei ght & Mark & \multicolumn{1}{c|}{ Conc. } \\
\hline 1 & 11.194 & 539827 & 17214 & $M$ & 50.164 \\
\hline 2 & 14.794 & 536305 & 10635 & $M$ & 49.836 \\
\hline Total & & 1076132 & 27848 & & 100.000 \\
\hline
\end{tabular}




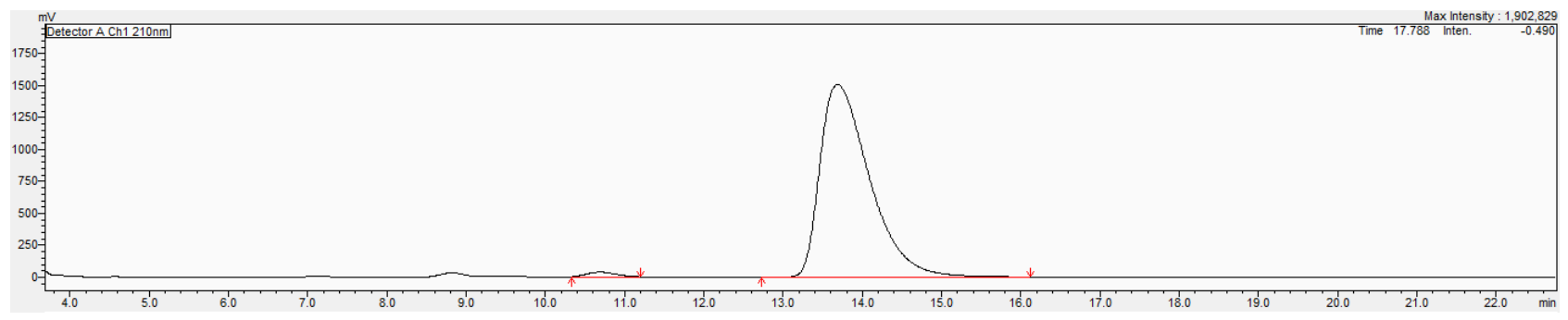

\begin{tabular}{|l|r|r|r|r|r|}
\hline Peak\# & Ret. Time & Area & Hei ght & Mark & \multicolumn{1}{|c|}{ Conc. } \\
\hline 1 & 10.687 & 921557 & 36640 & $M$ & 1.386 \\
\hline 2 & 13.688 & 65565240 & 1507311 & $M$ & 98.614 \\
\hline Total & & 66486796 & 1543951 & & 100.000 \\
\hline
\end{tabular}<smiles>O=C1O[C@](CBr)(c2cccc3ccccc23)CN(S)c2ccccc21</smiles>

(R)-3-(bromomethyl)-3-(naphthalen-1-yl)-1-tosyl-2,3-dihydrobenzo[e][1,4]oxazepin-5(1H)-one, 2k

$49.3 \mathrm{mg}, 92 \%$; white solid (starting from $45.7 \mathrm{mg}$ of $1 \mathbf{k})$; $\mathrm{MP} 248-249^{\circ} \mathrm{C}$; $[\alpha]_{D}^{25}=+8.5(c 1.0, \mathrm{MeOH}$, $96 \%$ ee); ${ }^{1} \mathrm{H}$ NMR $\left(\mathrm{CDCl}_{3}, 400 \mathrm{MHz}\right): \delta 8.36-6.43(\mathrm{~m}, 15 \mathrm{H}), 5.22\left(\mathrm{dd}, J_{l}=16.0 \mathrm{~Hz}, J_{2}=104.0 \mathrm{~Hz}\right.$, $2 \mathrm{H}), 4.29\left(\mathrm{dd}, J_{1}=12.0 \mathrm{~Hz}, J_{2}=232.0 \mathrm{~Hz}, 2 \mathrm{H}\right), 2.46(\mathrm{~s}, 3 \mathrm{H}) ;{ }^{13} \mathrm{C} \mathrm{NMR}\left(\mathrm{CDCl}_{3}, 100 \mathrm{MHz}\right): \delta 165.8$, 144.7, 135.4, 134.6, 134.3, 132.9, 131.8, 131.5, 130.4, 130.0, 129.0, 129.7,128.3, 127.6, 127.5, 127.1, 126.0, 125.0, 124.0, 84.1, 55.1, 37.2, 21.7; HRMS (TOF) $\mathrm{m} / \mathrm{z}:[\mathrm{M}+\mathrm{H}]^{+}$Calcd for $\mathrm{C}_{27} \mathrm{H}_{23} \mathrm{BrNO}_{4} \mathrm{~S}$ 536.7063; found 536.7064; HPLC (Daicel Chiralcel OD-H, $i$-PrOH/Hexane $=35 / 65,1.0 \mathrm{~mL} / \mathrm{min}$, $210 \mathrm{~nm}$ ) $\mathrm{t}_{1}=11.7 \mathrm{~min}$ (minor), $\mathrm{t}_{2}=12.6$ min (major).

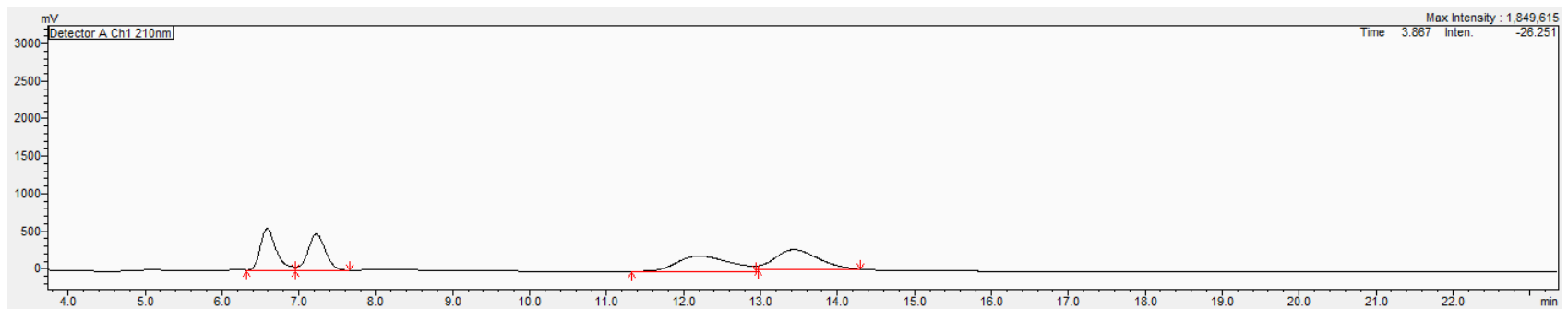

\begin{tabular}{|l|r|r|r|r|r|}
\hline Peak\# & Ret. Time & \multicolumn{1}{c|}{ Area } & Hei ght & Mark & \multicolumn{1}{c|}{ Conc. } \\
\hline 1 & 6.589 & 7950626 & 554967 & & 22.080 \\
\hline 2 & 7.227 & 7569348 & 483287 & $\mathrm{~V}$ & 21.022 \\
\hline 3 & 12.198 & 9725669 & 202098 & $\mathrm{M}$ & 27.010 \\
\hline 4 & 13.434 & 10761872 & 267108 & $\mathrm{M}$ & 29.888 \\
\hline Total & & 36007515 & 1507459 & & 100.000 \\
\hline
\end{tabular}




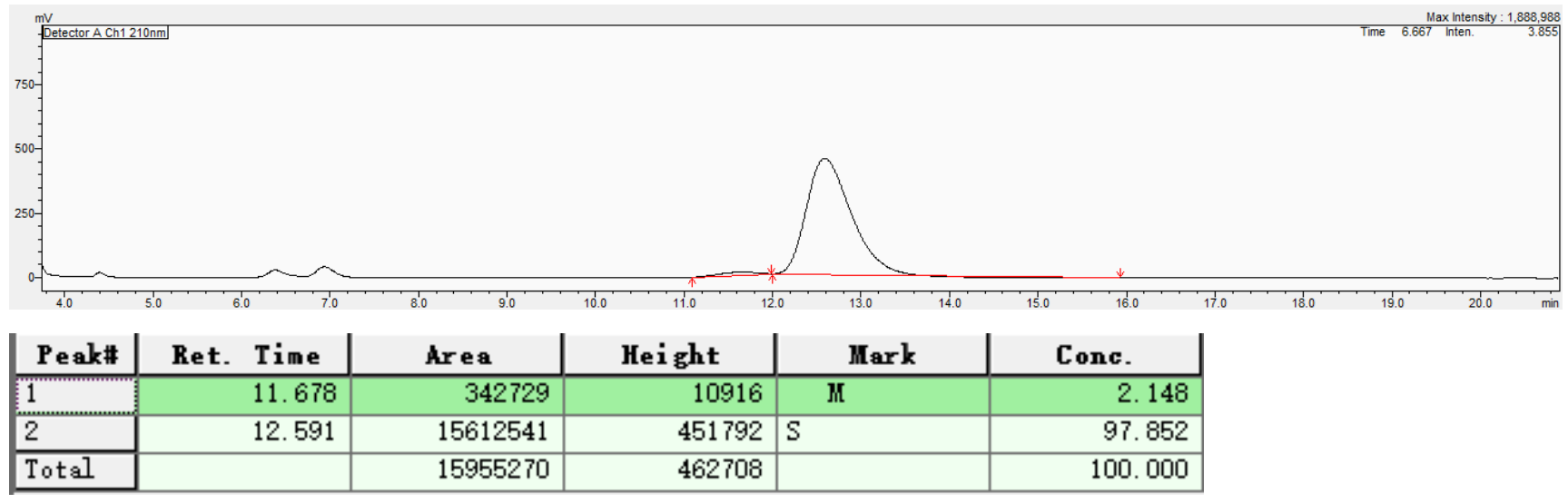<smiles>O=C1OC(CBr)(c2ccc3ccccc3c2)CNc2ccccc21</smiles>

(R)-3-(bromomethyl)-3-(naphthalen-2-yl)-1-tosyl-2,3-dihydrobenzo[e][1,4]oxazepin-5(1H)-one, $2 \mathrm{l}$

$52.5 \mathrm{mg}, 98 \%$; white solid (starting from $45.8 \mathrm{mg}$ of $1 \mathrm{l}$ ); $\mathrm{MP} 237-236^{\circ} \mathrm{C} ;[\alpha]_{D}^{25}=+10.9(c 1.0, \mathrm{MeOH}$, 99.5\% ee); ${ }^{1} \mathrm{H}$ NMR $\left(\mathrm{CDCl}_{3}, 400 \mathrm{MHz}\right): \delta 7.69-6.81(\mathrm{~m}, 15 \mathrm{H}), 5.21\left(\mathrm{dd}, J_{1}=16.0 \mathrm{~Hz}, J_{2}=132.0 \mathrm{~Hz}\right.$, $2 \mathrm{H}), 3.85\left(\mathrm{dd}, J_{l}=8.0 \mathrm{~Hz}, J_{2}=144.0 \mathrm{~Hz}, 2 \mathrm{H}\right), 2.46(\mathrm{~s}, 3 \mathrm{H}) ;{ }^{13} \mathrm{C} \mathrm{NMR}\left(\mathrm{CDCl}_{3}, 100 \mathrm{MHz}\right): \delta 166.3$, 144.6, 135.4, 134.7, 134.6, 133.0, 132.4, 132.3, 132.1, 131.6, 130.6, 129.9, 129.0, 128.6, 127.9, 127.7, 127.5, 127.1, 126.9, 126.0, 122.2, 82.4, 53.7, 40.0, 21.7; HRMS (TOF) m/z: $[\mathrm{M}+\mathrm{H}]^{+}$Calcd for $\mathrm{C}_{27} \mathrm{H}_{23} \mathrm{BrNO}_{4} \mathrm{~S}$ 536.7063; found 536.7062; HPLC (Daicel Chiralcel AD-H, $i$-PrOH/Hexane = 35/65, $1.0 \mathrm{~mL} / \mathrm{min}, 210 \mathrm{~nm}$ ) $\mathrm{t}_{1}=10.0 \mathrm{~min}$ (major), $\mathrm{t}_{2}=17.5 \mathrm{~min}$ (minor).

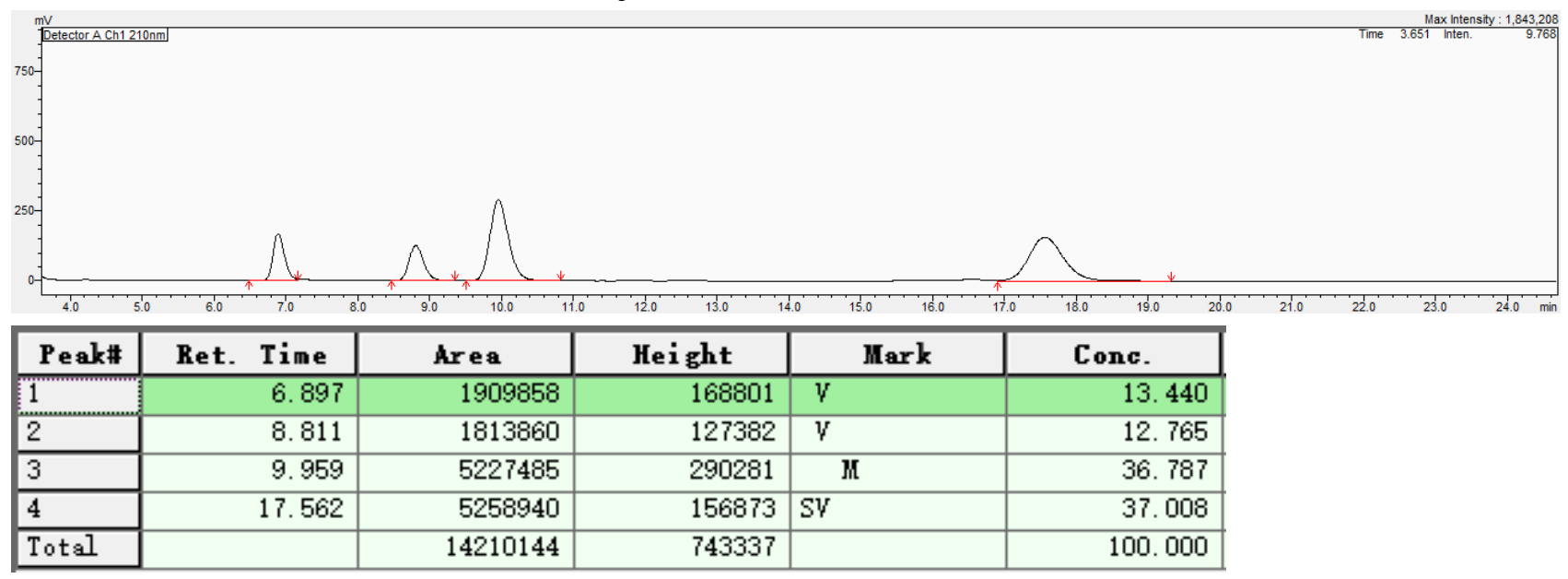




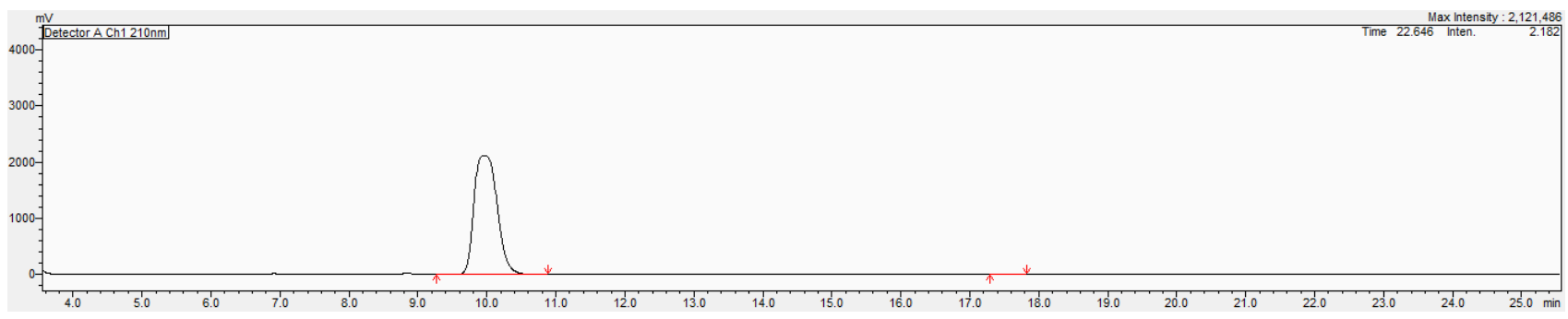

\begin{tabular}{|l|r|r|r|r|r|}
\hline Peak\# & Ret. Time & \multicolumn{1}{c|}{ Area } & \multicolumn{1}{c|}{ Height } & Mark & \multicolumn{1}{c|}{ Conc. } \\
\hline 1 & 9.963 & 48854187 & 2119511 & M & 99.735 \\
\hline 2 & 17.542 & 129990 & 6592 & M & 0.265 \\
\hline Total & & 48984176 & 2126104 & & 100.000 \\
\hline
\end{tabular}<smiles>Cc1cccc2c1N(C)CC(CBr)(c1ccccc1)OC2=O</smiles>

(R)-3-(bromomethyl)-9-methyl-3-phenyl-1-tosyl-2,3-dihydrobenzo[e][1,4]oxazepin-5(1H)-one, $2 \mathrm{~m}$

$44.9 \mathrm{mg}, 90 \%$; white solid (starting from $42.2 \mathrm{mg}$ of $1 \mathrm{~m})$; $\mathrm{MP} 199-200^{\circ} \mathrm{C}$; $[\alpha]_{D}^{25}=+4.1(c 1.0, \mathrm{MeOH}$, $48 \%$ ee $) ;{ }^{1} \mathrm{H} \mathrm{NMR}\left(\mathrm{CDCl}_{3}, 400 \mathrm{MHz}\right): \delta 7.64-6.81(\mathrm{~m}, 12 \mathrm{H}), 5.12\left(\mathrm{dd}, J_{1}=15.0 \mathrm{~Hz}, J_{2}=132.0 \mathrm{~Hz}\right.$, $2 \mathrm{H}), 3.72\left(\mathrm{dd}, J_{l}=12.0 \mathrm{~Hz}, J_{2}=108.0 \mathrm{~Hz}, 2 \mathrm{H}\right), 2.57(\mathrm{~s}, 3 \mathrm{H}), 2.49(\mathrm{~s}, 3 \mathrm{H}) ;{ }^{13} \mathrm{C} \mathrm{NMR}\left(\mathrm{CDCl}_{3}, 100\right.$ $\mathrm{MHz}): \delta 166.5,144.5,144.2,138.5,134.6,134.4,132.2,130.7,129.8,129.7,129.3,128.7,128.4$, 127.7, 125.6, 82.1, 53.3, 40.3, 21.7, 21.4; HRMS (TOF) m/z: $[\mathrm{M}+\mathrm{H}]^{+}$Calcd for $\mathrm{C}_{24} \mathrm{H}_{23} \mathrm{BrNO}_{4} \mathrm{~S}$ 500.6148; found 500.6147; HPLC (Daicel Chiralcel AD-H, $i$-PrOH/Hexane $=35 / 65,1.0 \mathrm{~mL} / \mathrm{min}$, $210 \mathrm{~nm}$ ) $\mathrm{t}_{1}=6.1 \mathrm{~min}$ (major), $\mathrm{t}_{2}=12.1 \mathrm{~min}$ (minor).

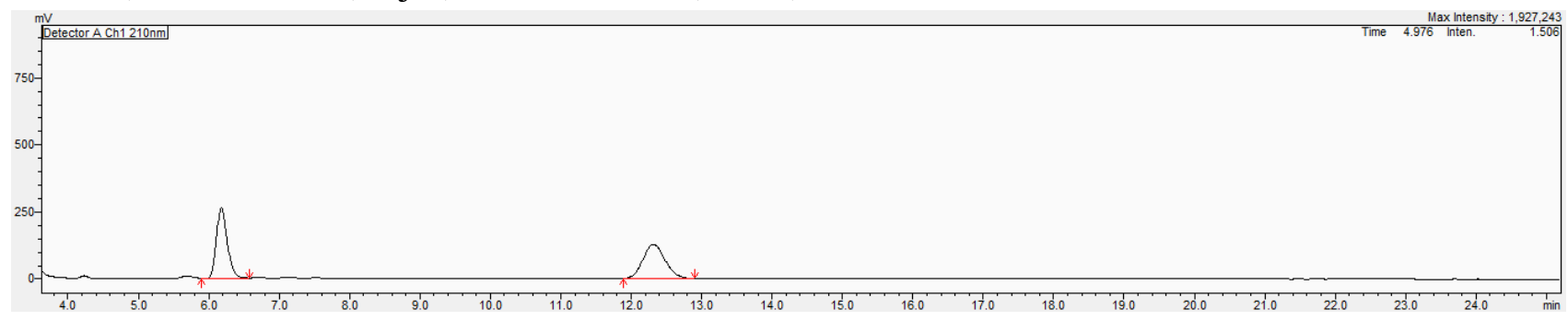

\begin{tabular}{|l|r|r|r|r|r|}
\hline Peak\# & Ret. Time & Area & Hei ght & Mark & \multicolumn{1}{|c|}{ Conc. } \\
\hline 1 & 6.179 & 2800161 & 262395 & $M$ & 50.037 \\
\hline 2 & 12.316 & 2796016 & 127422 & $M$ & 49.963 \\
\hline Total & & 5596177 & 389818 & & 100.000 \\
\hline
\end{tabular}

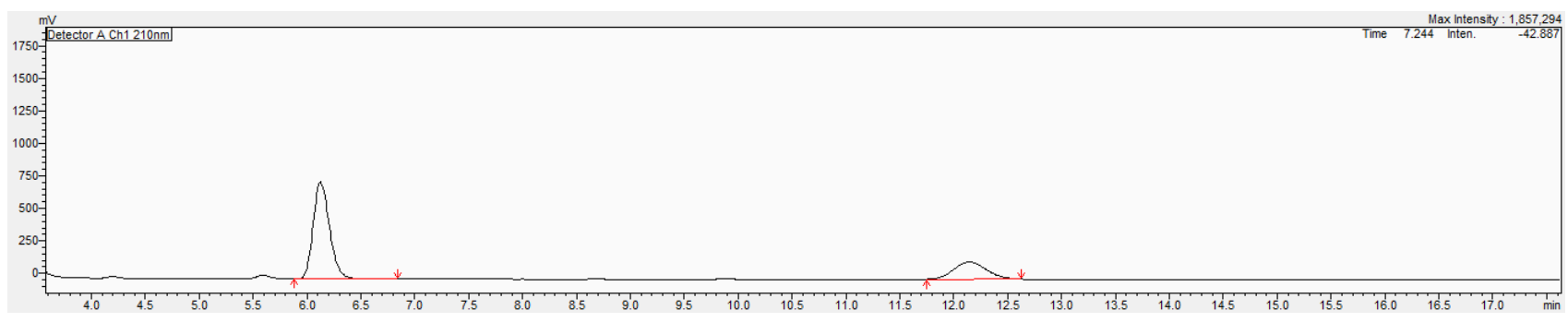

\begin{tabular}{|l|r|r|r|r|r|}
\hline Peak\# & Ret. Time & Area & Hei ght & Mark & \multicolumn{1}{c|}{ Conc. } \\
\hline 1 & 6.126 & 7895525 & 746400 & & 73.827 \\
\hline 2 & 12.142 & 2799058 & 132626 & M & 26.173 \\
\hline Total & & 10694583 & 879025 & & 100.000 \\
\hline
\end{tabular}


<smiles>Cc1ccc2c(c1)N(C)CC(CBr)(c1ccccc1)OC2=O</smiles>

(R)-3-(bromomethyl)-8-methyl-3-phenyl-1-tosyl-2,3-dihydrobenzo[e][1,4]oxazepin-5(1H)-one, 2n

$48.4 \mathrm{mg}$, 97\%; white solid (starting from $42.1 \mathrm{mg}$ of $1 \mathbf{n})$; $\mathrm{MP} 212-213^{\circ} \mathrm{C}$; $[\alpha]_{D}^{25}=+9.9(c 1.0$, $\mathrm{MeOH},>99.5 \%$ ee $) ;{ }^{1} \mathrm{H}$ NMR $\left(\mathrm{CDCl}_{3}, 100 \mathrm{MHz}\right): \delta 7.53-6.82(\mathrm{~m}, 12 \mathrm{H}), 5.11\left(\mathrm{dd}, J_{1}=15.0 \mathrm{~Hz}, J_{2}=\right.$ $123.0 \mathrm{~Hz}, 2 \mathrm{H}), 3.75\left(\mathrm{dd}, J_{l}=12.0 \mathrm{~Hz}, J_{2}=105.0 \mathrm{~Hz}, 2 \mathrm{H}\right), 2.45(\mathrm{~s}, 3 \mathrm{H}), 2.31(\mathrm{~s}, 3 \mathrm{H}) ;{ }^{13} \mathrm{C} \mathrm{NMR}$ $\left(\mathrm{CDCl}_{3}, 75 \mathrm{MHz}\right): \delta 166.5,144.5,144.2,138.5,134.6,134.4,132.2,130.7,129.8,129.7,129.3$, 128.7, 128.4, 127.7, 125.6, 82.1, 53.3, 40.3, 21.7, 21.4; HRMS (TOF) m/z: $[\mathrm{M}+\mathrm{H}]^{+}$Calcd for $\mathrm{C}_{24} \mathrm{H}_{23} \mathrm{BrNO}_{4} \mathrm{~S}$ 500.6148; found 500.6149; HPLC (Daicel Chiralcel OD-H, $i$-PrOH/Hexane = 30/60, $1.0 \mathrm{~mL} / \mathrm{min}, 210 \mathrm{~nm}$ ) $\mathrm{t}_{1}=7.9 \mathrm{~min}$ (minor), $\mathrm{t}_{2}=10.0 \mathrm{~min}$ (major).

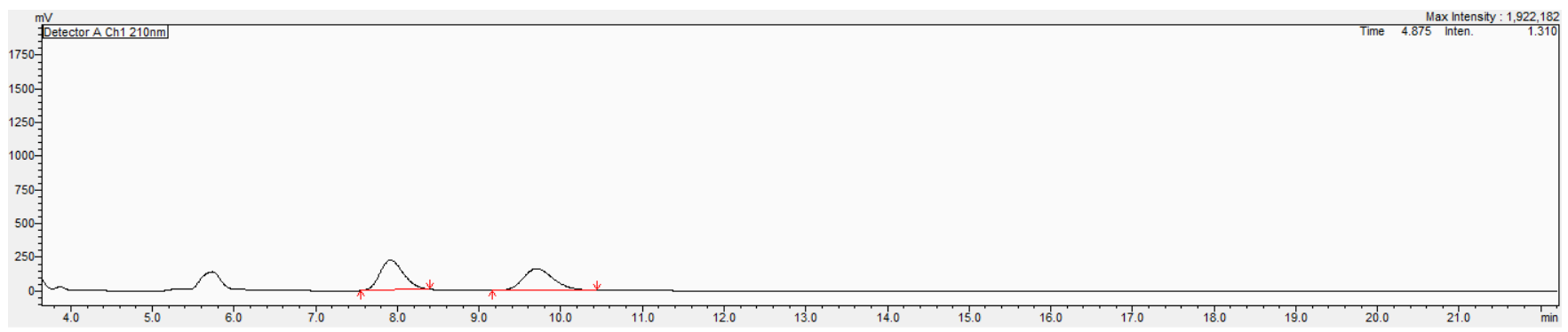

\begin{tabular}{|l|c|c|c|c|r|}
\hline Peak\# & Ret. Time & Area & Hei ght & Mark & \multicolumn{1}{|c|}{ Conc. } \\
\hline 1 & 7.914 & 4448003 & 221315 & M & 51.600 \\
\hline 2 & 9.705 & 4172238 & 160844 & M & 48.400 \\
\hline Total & & 8620241 & 382159 & & 100.000 \\
\hline
\end{tabular}

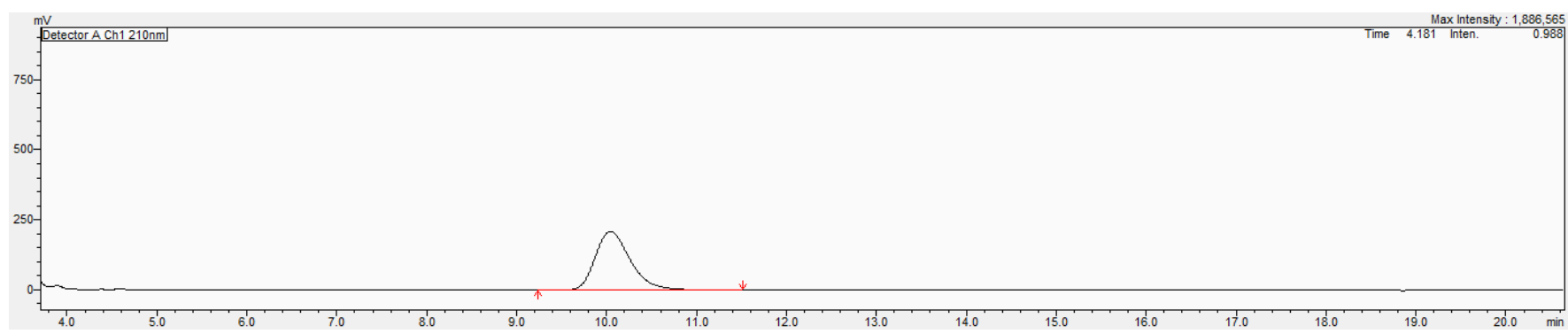

\begin{tabular}{|c|r|r|r|r|r|}
\hline Peak\# & Ret. Time & Area & Hei ght & Mark & \multicolumn{1}{c|}{ Conc. } \\
\hline 1 & 10.042 & 5698926 & 209977 & M & 100.000 \\
\hline Total & & 5698926 & 209977 & & 100.000 \\
\hline
\end{tabular}<smiles>COc1ccc2c(c1)C(=O)OC(CBr)(c1ccccc1)CN2C</smiles>

(R)-3-(bromomethyl)-7-methyl-3-phenyl-1-tosyl-2,3-dihydrobenzo[e][1,4]oxazepin-5(1H)-one, 20 $47.9 \mathrm{mg}, 96 \%$; white solid (starting from $42.1 \mathrm{mg}$ of 1o); $\mathrm{MP} 209-210^{\circ} \mathrm{C} ;[\alpha]_{D}^{25}=+11.7(c 1.0, \mathrm{MeOH}$, $98 \%$ ee); ${ }^{1} \mathrm{H} \mathrm{NMR}\left(\mathrm{CDCl}_{3}, 400 \mathrm{MHz}\right): \delta 7.53-6.77(\mathrm{~m}, 12 \mathrm{H}), 5.11\left(\mathrm{dd}, J_{l}=12.0 \mathrm{~Hz}, J_{2}=164.0 \mathrm{~Hz}\right.$, 
2H), $3.76\left(\mathrm{dd}, J_{l}=12.0 \mathrm{~Hz}, J_{2}=144.0 \mathrm{~Hz}, 2 \mathrm{H}\right), 2.45(\mathrm{~s}, 3 \mathrm{H}), 2.10(\mathrm{~s}, 3 \mathrm{H}) ;{ }^{13} \mathrm{C} \mathrm{NMR}\left(\mathrm{CDCl}_{3}, 100\right.$ $\mathrm{MHz}): \delta$ 166.6, 144.5, 139.2, 138.4, 134.7, 133.8, 132.0,131.9,131.6,131.0, 129.8, 128.7,128.2, 127.7, 125.7, 82.2, 53.4, 40.2, 21.7,20.6; HRMS (TOF) $\mathrm{m} / \mathrm{z}:[\mathrm{M}+\mathrm{H}]^{+}$Calcd for $\mathrm{C}_{24} \mathrm{H}_{23} \mathrm{BrNO}_{4} \mathrm{~S}$ 500.6148; found 500.6147; HPLC (Daicel Chiralcel OD-H, $i$-PrOH/Hexane $=30 / 60,1.0 \mathrm{~mL} / \mathrm{min}$, $210 \mathrm{~nm}$ ) $\mathrm{t}_{1}=9.1 \mathrm{~min}$ (major), $\mathrm{t}_{2}=12.1 \mathrm{~min}$ (minor).
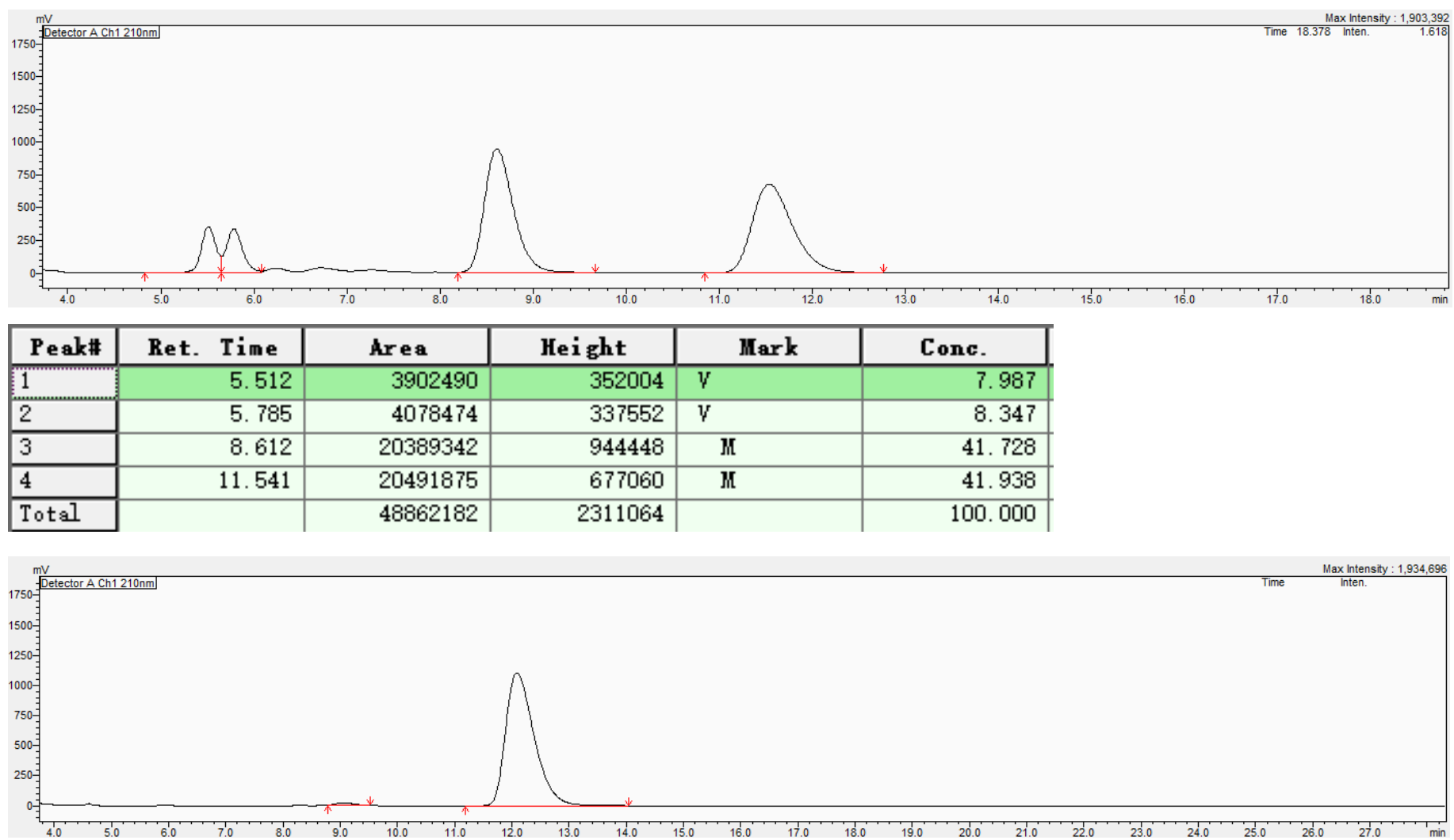

\begin{tabular}{|l|r|r|r|r|r|}
\hline Peak\# & Ret. Time & \multicolumn{1}{|c|}{ Area } & Hei ght & \multicolumn{1}{|c|}{ Mark } & \multicolumn{1}{c|}{ Conc. } \\
\hline 1 & 9.058 & 451366 & 20633 & M & 1.167 \\
\hline 2 & 12.088 & 38223919 & 1106195 & M & 98.833 \\
\hline Total & & 38675285 & 1126828 & & 100.000 \\
\hline
\end{tabular}<smiles>COc1ccc2c(c1)N(C)CC(CBr)(c1ccccc1)OC2=O</smiles>

(R)-3-(bromomethyl)-8-methoxy-3-phenyl-1-tosyl-2,3-dihydrobenzo[e][1,4]oxazepin-5(1H)-one, 2p $50.5 \mathrm{mg}, 98 \%$; white solid (starting from $43.8 \mathrm{mg}$ of $1 \mathbf{p})$; MP $237-237^{\circ} \mathrm{C}$; $[\alpha]_{D}^{25}=+6.4(c 1.0, \mathrm{MeOH}$, $99 \%$ ee); ${ }^{1} \mathrm{H}$ NMR $\left(\mathrm{CDCl}_{3}, 400 \mathrm{MHz}\right): \delta 7.53-6.54(\mathrm{~m}, 12 \mathrm{H}), 5.10\left(\mathrm{dd}, J_{l}=12.0 \mathrm{~Hz}, J_{2}=164.0 \mathrm{~Hz}\right.$, $2 \mathrm{H}), 3.80(\mathrm{~s}, 3 \mathrm{H}), 3.74\left(\mathrm{dd}, J_{l}=8.0 \mathrm{~Hz}, J_{2}=136.0 \mathrm{~Hz}, 2 \mathrm{H}\right), 2.44(\mathrm{~s}, 3 \mathrm{H}) ;{ }^{13} \mathrm{C} \mathrm{NMR}\left(\mathrm{CDCl}_{3}, 100\right.$ $\mathrm{MHz}): \delta 166.2,163.1,144.6,138.5,136.5,134.5,132.4,129.8,128.8,128.4,127.7,125.5,124.3$, 116.5, 115.1, 82.1, 55.8, 53.2, 40.4, 21.7; HRMS (TOF) m/z: $[\mathrm{M}+\mathrm{H}]^{+}$Calcd for $\mathrm{C}_{24} \mathrm{H}_{23} \mathrm{BrNO}_{5} \mathrm{~S}$ 516.2980; found 516.2981; HPLC (Daicel Chiralcel AD-H, $i$-PrOH/Hexane $=35 / 65,1.0 \mathrm{~mL} / \mathrm{min}$, $210 \mathrm{~nm}$ ) $\mathrm{t}_{1}=7.3 \mathrm{~min}$ (major), $\mathrm{t}_{2}=11.6 \mathrm{~min}$ (minor). 


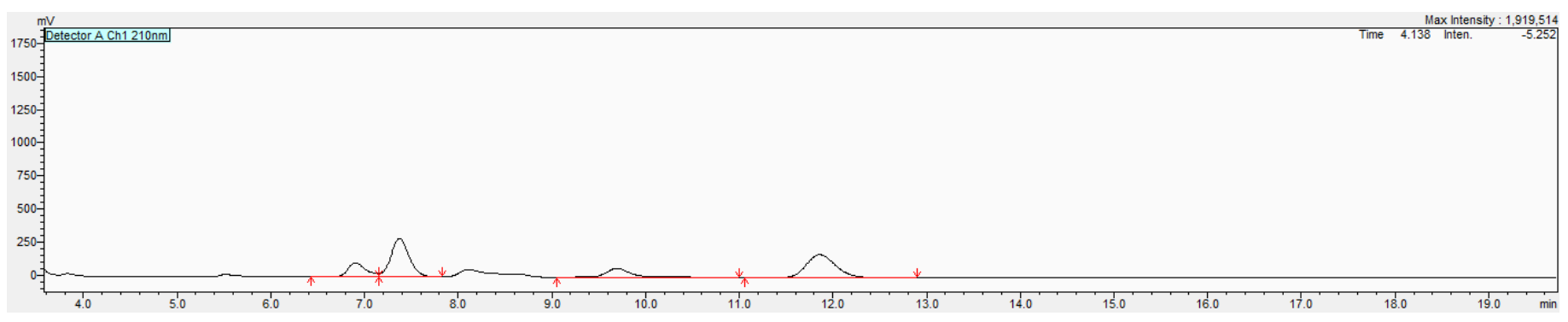

\begin{tabular}{|l|r|r|r|r|r|}
\hline Peak\# & Ret. Time & Area & \multicolumn{1}{c|}{ Height } & \multicolumn{1}{c|}{ Mark } & \multicolumn{1}{c|}{ Conc. } \\
\hline 1 & 6.904 & 1469974 & 108110 & & 13.793 \\
\hline 2 & 7.375 & 3942563 & 290261 & $V$ & 36.994 \\
\hline 3 & 9.695 & 1405516 & 67620 & $\mathrm{~S}$ & 13.188 \\
\hline 4 & 11.857 & 3839195 & 174080 & & 36.024 \\
\hline Total & & 10657247 & 640071 & & 100.000 \\
\hline
\end{tabular}

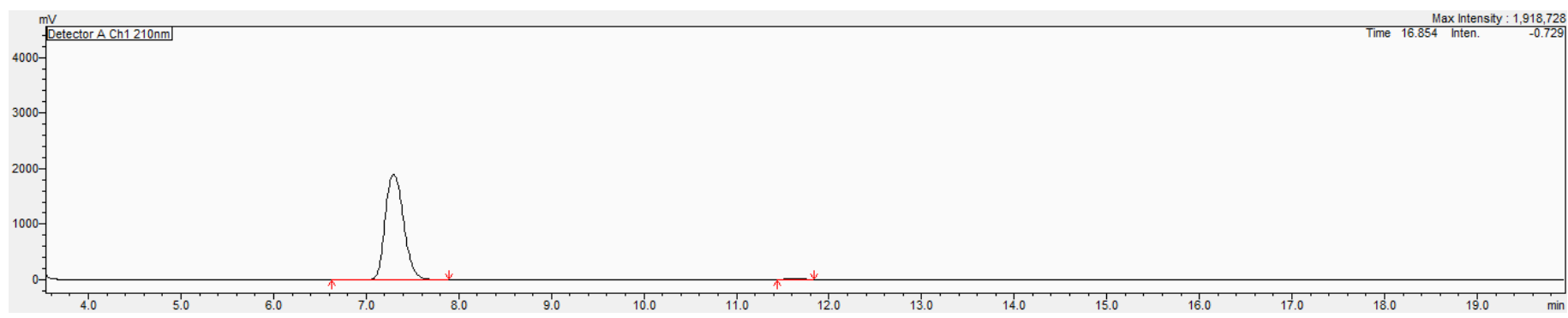

\begin{tabular}{|l|r|r|r|r|r|}
\hline Peak\# & Ret. Time & \multicolumn{1}{c|}{ Area } & \multicolumn{1}{c|}{ Height } & \multicolumn{1}{c|}{ Mark } & \multicolumn{1}{c|}{ Conc. } \\
\hline 1 & 7.296 & 26068862 & 1891014 & M & 99.418 \\
\hline 2 & 11.621 & 152514 & 10835 & M & 0.582 \\
\hline Total & & 26221376 & 1901848 & & 100.000 \\
\hline
\end{tabular}<smiles>O=C1OC(CBr)(c2ccccc2)CN(S)c2cc(F)ccc21</smiles>

(R)-3-(bromomethyl)-8-fluoro-3-phenyl-1-tosyl-2,3-dihydrobenzo[e][1,4]oxazepin-5(1H)-one, 2q $49.8 \mathrm{mg}$, 99\%; white solid (starting from $42.5 \mathrm{mg}$ of 1q); $\mathrm{MP} 204-205^{\circ} \mathrm{C} ;[\alpha]_{D}^{25}=+8.5(c 1.0, \mathrm{MeOH}$, $99 \%$ ee); ${ }^{1} \mathrm{H}$ NMR $\left(\mathrm{CDCl}_{3}, 300 \mathrm{MHz}\right): \delta 7.53-6.72(\mathrm{~m}, 12 \mathrm{H}), 5.11\left(\mathrm{dd}, J_{l}=12.0 \mathrm{~Hz}, J_{2}=164.0 \mathrm{~Hz}\right.$, $2 \mathrm{H}), 3.77\left(\mathrm{dd}, J_{l}=12.0 \mathrm{~Hz}, J_{2}=140.0 \mathrm{~Hz}, 2 \mathrm{H}\right), 2.45(\mathrm{~s}, 3 \mathrm{H}) ;{ }^{13} \mathrm{C} \mathrm{NMR}\left(\mathrm{CDCl}_{3}, 75 \mathrm{MHz}\right): \delta 165.8(\mathrm{~d}$, $J=250.0 \mathrm{~Hz}), 1654.4,144.9,138.2,136.8(\mathrm{~d}, J=11.0 \mathrm{~Hz}), 134.3,132.8,132.7,130.0,128.9,128.7$, 128.5, 127.6, 125.6, $119.1(\mathrm{~d}, J=23.0 \mathrm{~Hz}), 116.4(\mathrm{~d}, J=22.0 \mathrm{~Hz}), 82.3,53.3,40.1,21.7$; HRMS (TOF) m/z: $[\mathrm{M}+\mathrm{H}]^{+}$Calcd for $\mathrm{C}_{23} \mathrm{H}_{20} \mathrm{BrFNO}_{4} \mathrm{~S}$ 504.3962; found 504.3963; HPLC (Daicel Chiralcel $\mathrm{AD}-\mathrm{H}, i-\mathrm{PrOH} / \mathrm{Hexane}=35 / 65,1.0 \mathrm{~mL} / \mathrm{min}, 210 \mathrm{~nm}$ ) $\mathrm{t}_{1}=6.2 \mathrm{~min}$ (major), $\mathrm{t}_{2}=7.6 \mathrm{~min}$ (minor). 


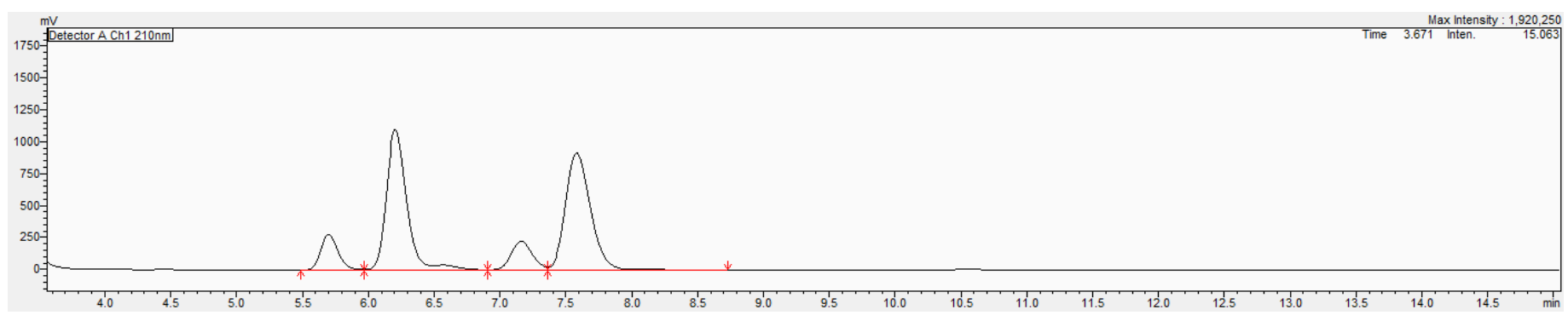

\begin{tabular}{|l|r|r|r|r|r|}
\hline Peak\# & Ret. Time & Area & \multicolumn{1}{c|}{ Hei ght } & Mark & Conc. \\
\hline 1 & 5.702 & 2502728 & 278126 & & 8.636 \\
\hline 2 & 6.206 & 12004228 & 1100240 & SV & 41.423 \\
\hline 3 & 7.161 & 2481068 & 225262 & $V$ & 8.561 \\
\hline 4 & 7.584 & 11991746 & 916320 & SV & 41.380 \\
\hline Total & & 28979771 & 2519949 & & 100.000 \\
\hline
\end{tabular}

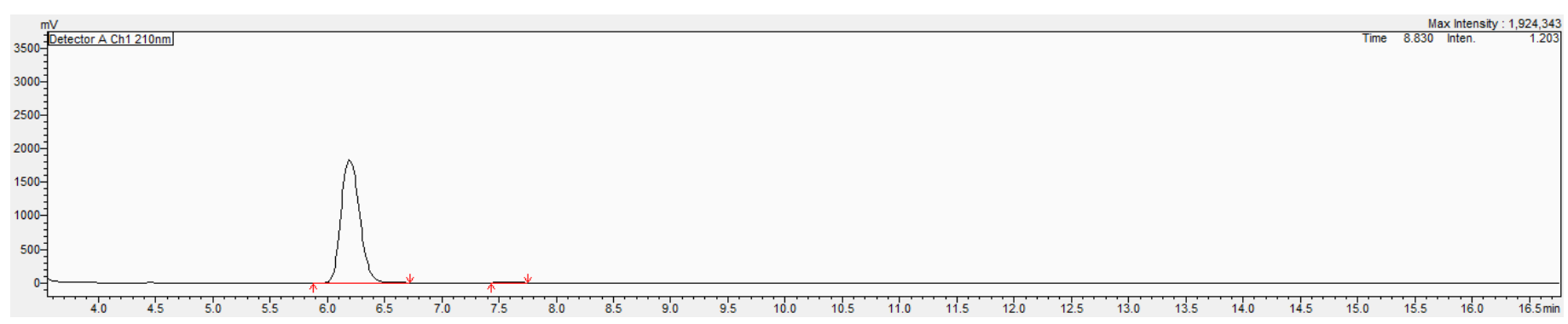

\begin{tabular}{|l|r|r|r|r|r|}
\hline Peak\# & Ret. Time & \multicolumn{1}{c|}{ Area } & \multicolumn{1}{c|}{ Hei ght } & \multicolumn{1}{|c|}{ Mark } & Conc. \\
\hline 1 & 6.194 & 20764107 & 1829500 & M & 99.605 \\
\hline 2 & 7.577 & 82289 & 8091 & M & 0.395 \\
\hline Total & & 20846396 & 1837591 & & 100.000 \\
\hline
\end{tabular}<smiles>O=C1OC(CBr)(c2ccccc2)CN(S)c2cc(Cl)ccc21</smiles>

(R)-3-(bromomethyl)-8-chloro-3-phenyl-1-tosyl-2,3-dihydrobenzo[e][1,4]oxazepin-5(1H)-one, $2 \mathrm{r}$ $51.4 \mathrm{mg}$, 99\%; white solid (starting from $44.2 \mathrm{mg}$ of $1 \mathbf{r}$ ); $\mathrm{MP} 213-214^{\circ} \mathrm{C} ;[\alpha]_{D}^{25}=+12.4(\mathrm{c} 1.0, \mathrm{MeOH}$, 99\% ee); ${ }^{1} \mathrm{H} \mathrm{NMR}\left(\mathrm{CDCl}_{3}, 400 \mathrm{MHz}\right): \delta 7.54-6.90(\mathrm{~m}, 12 \mathrm{H}), 5.14\left(\mathrm{dd}, J_{l}=12.0 \mathrm{~Hz}, J_{2}=180.0 \mathrm{~Hz}\right.$, $2 \mathrm{H}), 3.77\left(\mathrm{dd}, J_{1}=12.0 \mathrm{~Hz}, J_{2}=144.0 \mathrm{~Hz}, 2 \mathrm{H}\right), 2.45(\mathrm{~s}, 3 \mathrm{H}) ;{ }^{13} \mathrm{C} \mathrm{NMR}\left(\mathrm{CDCl}_{3}, 100 \mathrm{MHz}\right): \delta 165.5$, 144.9, 138.8, 138.1, 135.7, 134.3, 131.8, 131.6, 130.6, 130.0, 129.1, 128.8, 127.7, 125.7, 82.3, 53.2, 40.0, 21.7; HRMS (TOF) $\mathrm{m} / \mathrm{z}$ : $[\mathrm{M}+\mathrm{H}]^{+}$Calcd for $\mathrm{C}_{23} \mathrm{H}_{20} \mathrm{BrClNO}_{4} \mathrm{~S}$ 520.1744; found 520.1743; HPLC (Daicel Chiralcel AD-H, $i$-PrOH/Hexane = 35/65, $1.0 \mathrm{~mL} / \mathrm{min}, 210 \mathrm{~nm}$ ) $\mathrm{t}_{1}=6.1 \mathrm{~min}$ (major), $\mathrm{t}_{2}=7.5 \mathrm{~min}$ (minor). 


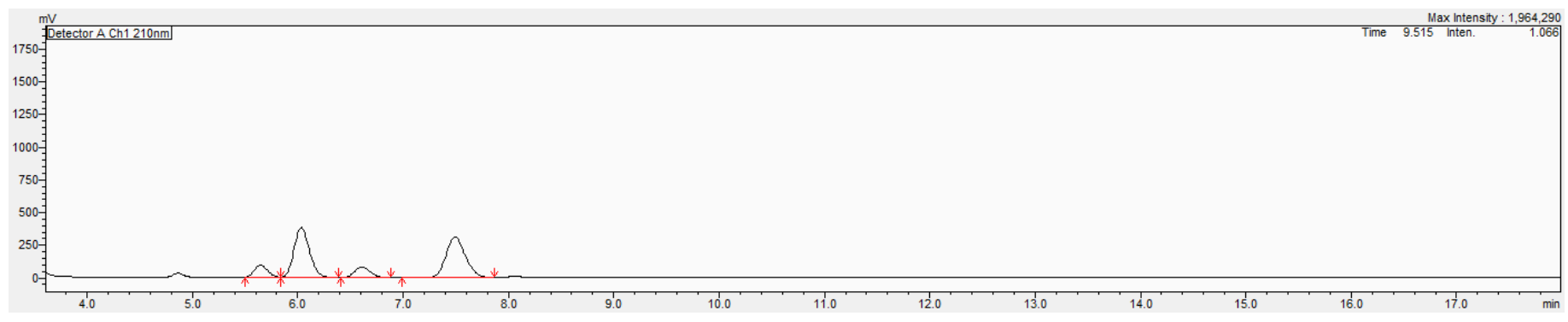

\begin{tabular}{|l|r|r|r|r|r|}
\hline Peak\# & Ret. Time & \multicolumn{1}{c|}{ Area } & Height & Mark & \multicolumn{1}{c|}{ Conc. } \\
\hline 1 & 5.644 & 808501 & 92687 & M & 8.382 \\
\hline 2 & 6.033 & 3984221 & 386314 & & 41.305 \\
\hline 3 & 6.609 & 863835 & 83197 & M & 8.956 \\
\hline 4 & 7.494 & 3989294 & 310512 & & 41.358 \\
\hline Total & & 9645850 & 872710 & & 100.000 \\
\hline
\end{tabular}

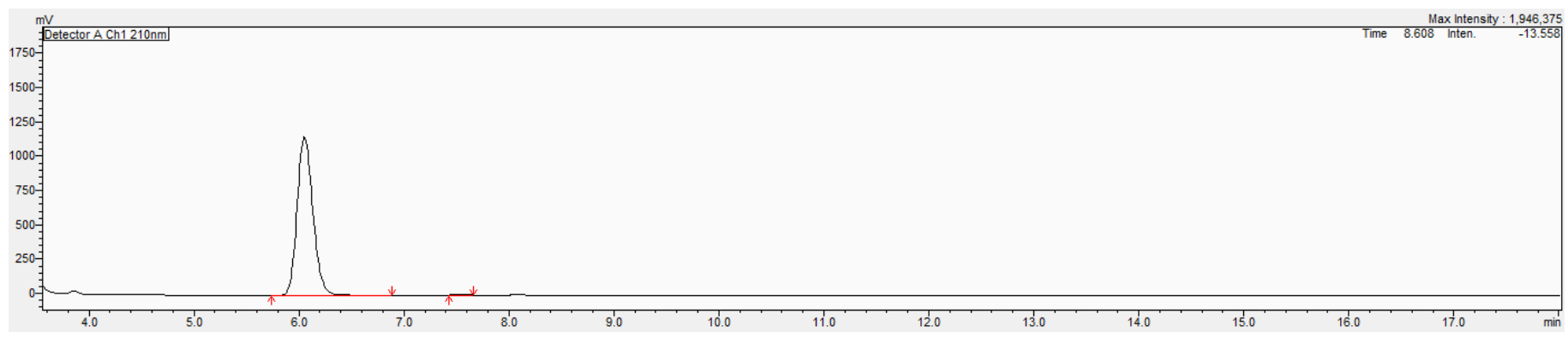

\begin{tabular}{|l|r|r|r|r|r|}
\hline Peak\# & Ret. Time & \multicolumn{1}{c|}{ Area } & Hei ght & \multicolumn{1}{c|}{ Mark } & \multicolumn{1}{c|}{ Conc. } \\
\hline 1 & 6.052 & 12006431 & 1151982 & & 99.808 \\
\hline 2 & 7.543 & 23155 & 2774 & M & 0.192 \\
\hline Total & & 12029586 & 1154756 & & 100.000 \\
\hline
\end{tabular}<smiles>CN1CC(CBr)(c2ccccc2)OC(=O)c2ccc(Br)cc21</smiles>

(R)-8-bromo-3-(bromomethyl)-3-phenyl-1-tosyl-2,3-dihydrobenzo[e][1,4]oxazepin-5(1H)-one, $2 \mathrm{~s}$

$55.7 \mathrm{mg}$, 99\%; yellow solid (starting from $48.5 \mathrm{mg}$ of $1 \mathrm{~s})$; MP $238-239^{\circ} \mathrm{C}$; $[\alpha]_{D}^{25}=+11.3(c 1.0$, $\mathrm{MeOH},>99.5 \%$ ee $) ;{ }^{1} \mathrm{H}$ NMR $\left(\mathrm{CDCl}_{3}, 400 \mathrm{MHz}\right): \delta 7.69-6.82(\mathrm{~m}, 12 \mathrm{H}), 5.11\left(\mathrm{dd}, J_{l}=12.0 \mathrm{~Hz}, J_{2}=\right.$ $172.0 \mathrm{~Hz}, 2 \mathrm{H}), 3.76\left(\mathrm{dd}, J_{1}=8.0 \mathrm{~Hz}, J_{2}=140.0 \mathrm{~Hz}, 2 \mathrm{H}\right), 2.45(\mathrm{~s}, 3 \mathrm{H}) ;{ }^{13} \mathrm{C} \mathrm{NMR}\left(\mathrm{CDCl}_{3}, 100 \mathrm{MHz}\right)$ : $\delta 165.7,144.9,138.1,135.6,134.6,132.0,131.6,131.1,130.0,129.0,128.8,127.7,126.9,125.7$, 82.3, 53.2, 40.0, 21.7; HRMS (TOF) $\mathrm{m} / \mathrm{z}$ : $[\mathrm{M}+\mathrm{H}]^{+}$Calcd for $\mathrm{C}_{23} \mathrm{H}_{20} \mathrm{Br}_{2} \mathrm{NO}_{4} \mathrm{~S}$ 564.3217; found 564.3218; HPLC (Daicel Chiralcel AD-H, $i$-PrOH/Hexane $=35 / 65,1.0 \mathrm{~mL} / \mathrm{min}, 210 \mathrm{~nm}$ ) $\mathrm{t}_{1}=6.4$ $\min$ (major), $\mathrm{t}_{2}=7.6 \mathrm{~min}$ (minor). 


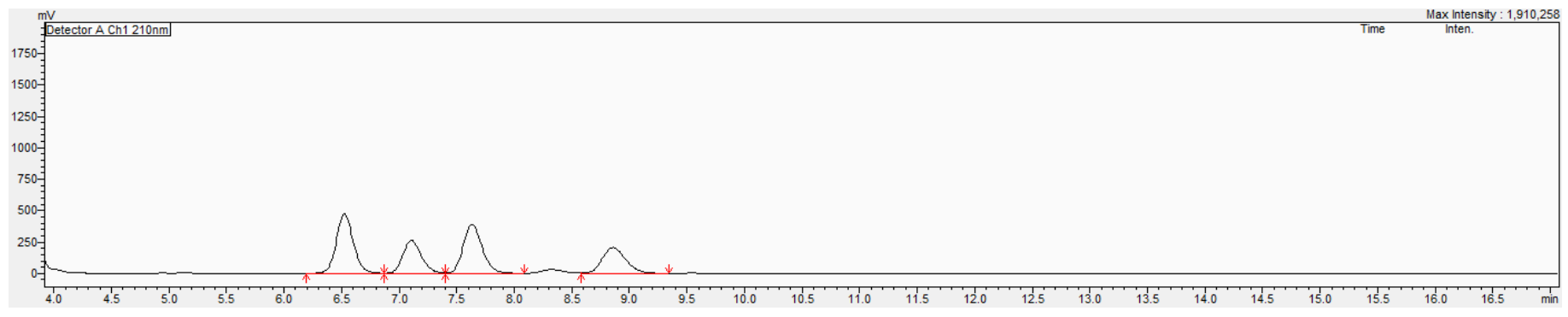

\begin{tabular}{|l|r|r|r|r|r|}
\hline Peak\# & Ret. Time & \multicolumn{1}{c|}{ Area } & \multicolumn{1}{c|}{ Hei ght } & \multicolumn{1}{c|}{ Mark } & \multicolumn{1}{c|}{ Conc. } \\
\hline 1 & 6.524 & 4879602 & 476883 & V & 31.956 \\
\hline 2 & 7.107 & 2940113 & 261457 & $V$ & 19.255 \\
\hline 3 & 7.632 & 4501158 & 391221 & $V$ & 29.478 \\
\hline 4 & 8.858 & 2948675 & 206173 & $V$ & 19.311 \\
\hline Total & & 15269548 & 1335733 & & 100.000 \\
\hline
\end{tabular}

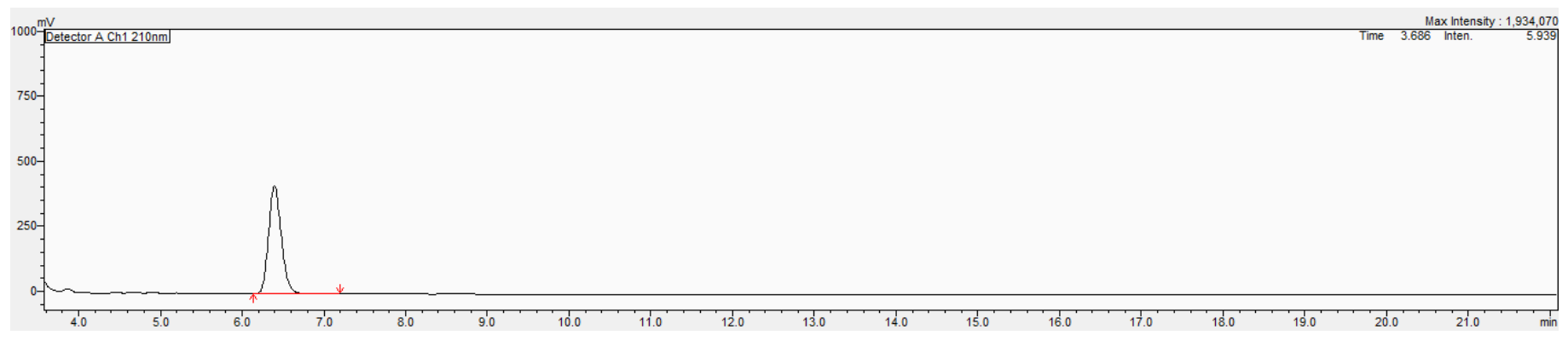

\begin{tabular}{|l|c|r|r|r|r|}
\hline Peak\# & Ret. Time & \multicolumn{1}{c|}{ Area } & \multicolumn{1}{c|}{ Kei ght } & \multicolumn{1}{c|}{ Mark } & \multicolumn{1}{c|}{ Conc. } \\
\hline 1 & 6.395 & 4530845 & 416082 & V & 100.000 \\
\hline Total & & 4530845 & 416082 & & 100.000 \\
\hline
\end{tabular}<smiles>CN1C[C@@](CI)(c2ccccc2)OC(=O)c2ccccc21</smiles>

\section{(R)-3-(iodomethyl)-3-phenyl-1-tosyl-2,3-dihydrobenzo[e][1,4]oxazepin-5(1H)-one, 3a}

$50.6 \mathrm{mg}$, 95\%; yellow solid (starting from $40.6 \mathrm{mg}$ of $1 \mathrm{a})$; $\mathrm{MP} 224-225^{\circ} \mathrm{C} ;[\alpha]_{D}^{25}=+9.0(c 1.0, \mathrm{MeOH}$, $93 \%$ ee); ${ }^{1} \mathrm{H} \mathrm{NMR}\left(\mathrm{CDCl}_{3}, 400 \mathrm{MHz}\right): \delta 7.54-6.98(\mathrm{~m}, 13 \mathrm{H}), 4.99\left(\mathrm{dd}, J_{l}=12.0 \mathrm{~Hz}, J_{2}=88.0 \mathrm{~Hz}\right.$,

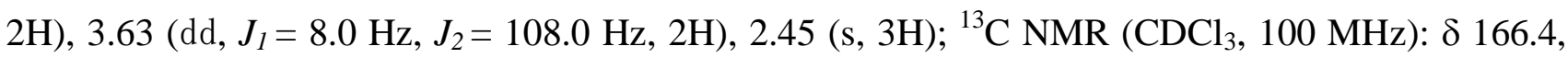
144.6, 138.3, 134.5, 134.4, 133.0, 132.2, 131.8, 130.8, 129.9, 128.8, 128.3, 127.7, 125.5, 81.4, 56.3, 21.7, 15.2; HRMS (TOF) $\mathrm{m} / \mathrm{z}:[\mathrm{M}+\mathrm{H}]^{+}$Calcd for $\mathrm{C}_{23} \mathrm{H}_{21} \mathrm{INO}_{4} \mathrm{~S}$ 534.4982; found 534.4983; HPLC (Daicel Chiralcel OD-H, $i-\mathrm{PrOH} / \mathrm{Hexane}=35 / 65,1.0 \mathrm{~mL} / \mathrm{min}, 210 \mathrm{~nm}) \mathrm{t}_{1}=8.6 \mathrm{~min}($ minor $), \mathrm{t}_{2}=$ $11.8 \mathrm{~min}$ (major). 


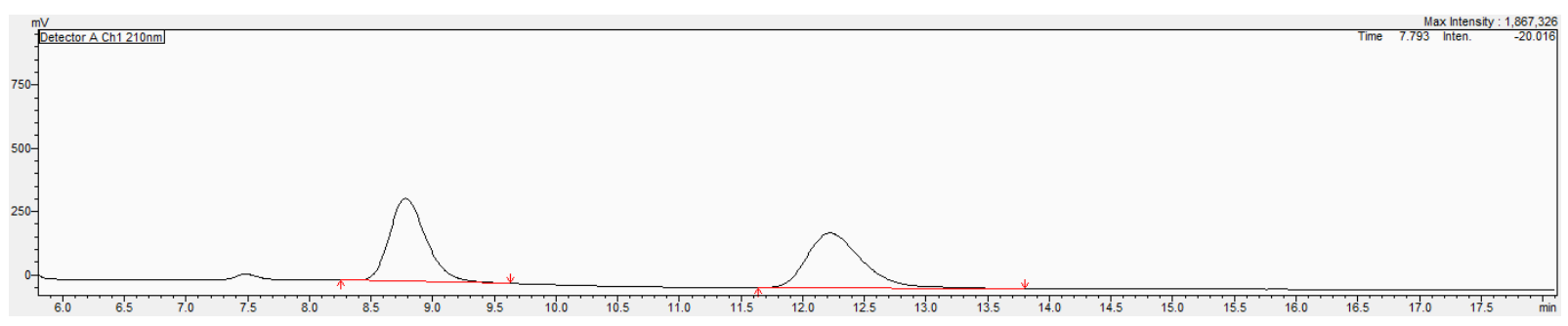

\begin{tabular}{|l|r|r|r|r|r|}
\hline Peak\# & Ret. Time & Area & Mei ght & Mark & \multicolumn{1}{c|}{ Conc. } \\
\hline 1 & 8.780 & 6798495 & 328792 & M & 50.328 \\
\hline 2 & 12.222 & 6709974 & 216994 & & 49.672 \\
\hline Total & & 13508469 & 545786 & & 100.000 \\
\hline
\end{tabular}

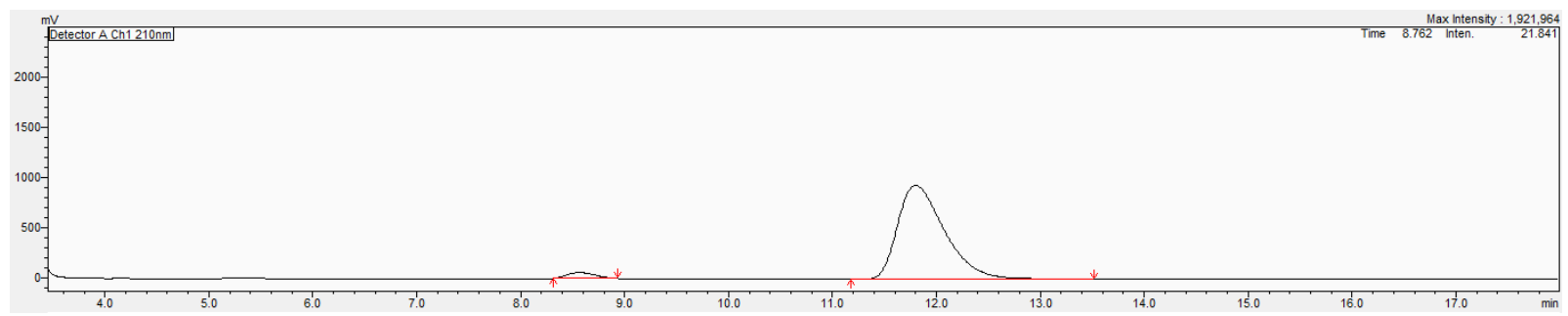

\begin{tabular}{|l|r|r|r|r|r|}
\hline Peak\# & Ret. Time & Area & Height & Mark & \multicolumn{1}{c|}{ Conc. } \\
\hline 1 & 8.566 & 1078101 & 57774 & $M$ & 3.550 \\
\hline 2 & 11.801 & 29294990 & 930376 & $V$ & 96.450 \\
\hline Total & & 30373091 & 988151 & & 100.000 \\
\hline
\end{tabular}<smiles>CN1CC(CCl)(c2ccccc2)OC(=O)c2ccccc21</smiles>

\section{(R)-3-(chloromethyl)-3-phenyl-1-tosyl-2,3-dihydrobenzo[e][1,4]oxazepin-5(1H)-one, 4a}

$42.3 \mathrm{mg}$, 96\%; white solid (starting from $40.7 \mathrm{mg}$ of 1a); MP $167-168^{\circ} \mathrm{C}$; $[\alpha]_{D}^{25}=-33.0(c 1.0, \mathrm{MeOH}$, 97\% ee); ${ }^{1} \mathrm{H} \mathrm{NMR}\left(\mathrm{CDCl}_{3}, 400 \mathrm{MHz}\right): \delta 7.51-6.97(\mathrm{~m}, 13 \mathrm{H}), 5.14\left(\mathrm{dd}, J_{l}=12.0 \mathrm{~Hz}, J_{2}=180.0 \mathrm{~Hz}\right.$,

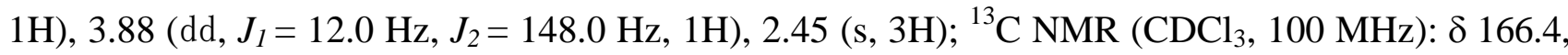
144.6, 138.2, 134.6, 133.0, 132.2, 131.8, 130.7, 129.9, 128.8, 128.5, 127.6, 125.7, 83.0, 52.2, 51.8, 21.7; HRMS (TOF) m/z: $[\mathrm{M}+\mathrm{H}]^{+}$Calcd for $\mathrm{C}_{23} \mathrm{H}_{21} \mathrm{ClNO}_{4} \mathrm{~S}$ 442.5935; found 442.5936; HPLC (Daicel Chiralcel OD-H, $i$-PrOH/Hexane $=35 / 65,1.0 \mathrm{~mL} / \mathrm{min}, 210 \mathrm{~nm}) \mathrm{t}_{1}=9.1 \mathrm{~min}(\operatorname{minor}), \mathrm{t}_{2}=$ 12.7 min (major). 


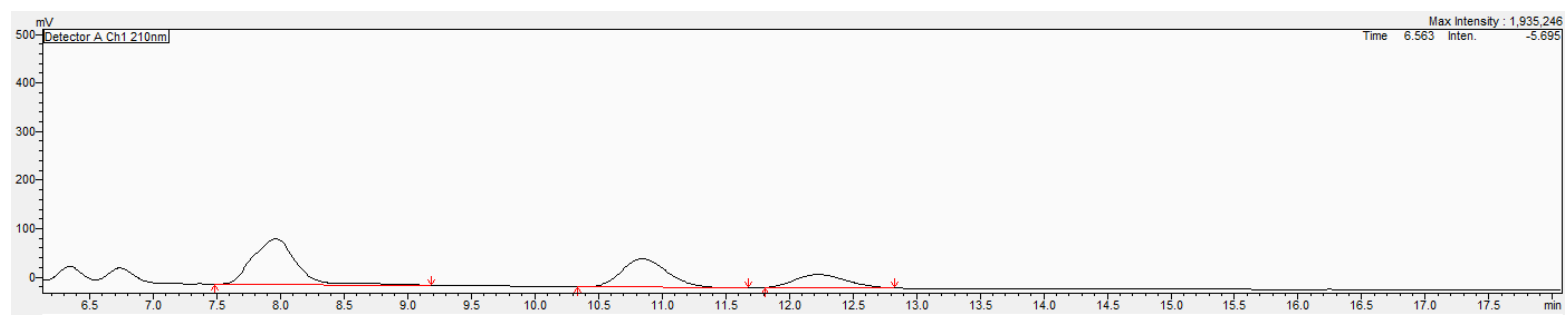

\begin{tabular}{|l|r|r|r|r|r|}
\hline Peak\# & Ret. Time & \multicolumn{1}{c|}{ Area } & Hei ght & \multicolumn{1}{c|}{ Mark } & \multicolumn{1}{c|}{ Conc. } \\
\hline 1 & 7.963 & 2236602 & 94359 & SV & 49.709 \\
\hline 2 & 10.845 & 1498001 & 59354 & & 33.294 \\
\hline 3 & 12.225 & 764770 & 27254 & M & 16.997 \\
\hline Total & & 4499373 & 180967 & & 100.000 \\
\hline
\end{tabular}

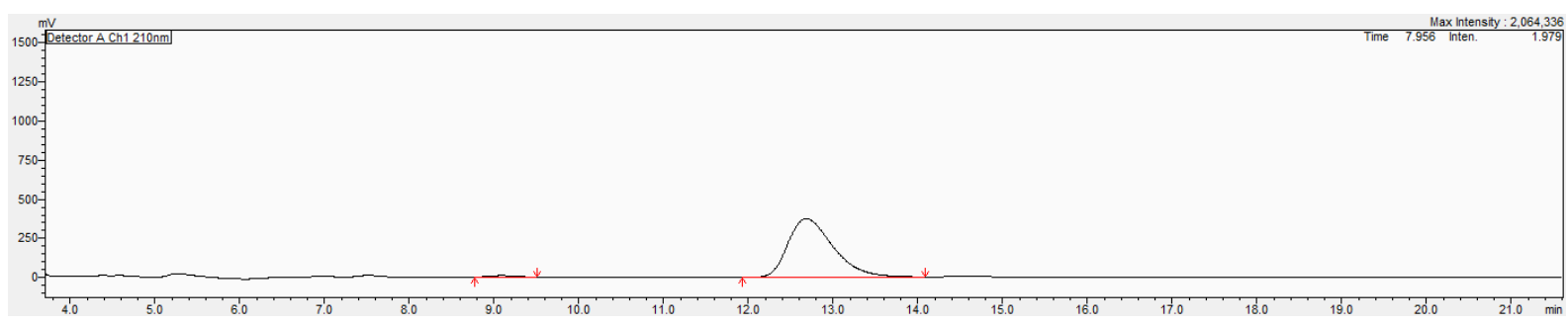

\begin{tabular}{|l|r|r|r|r|r|}
\hline Peak\# & Ret. Time & Area & Hei ght & \multicolumn{1}{c|}{ Mark } & \multicolumn{1}{c|}{ Conc. } \\
\hline 1 & 9.088 & 209119 & 9522 & M & 1.472 \\
\hline 2 & 12.683 & 13996731 & 375944 & $V$ & 98.528 \\
\hline Total & & 14205849 & 385465 & & 100.000 \\
\hline
\end{tabular}<smiles></smiles>

(S)-4-(bromomethyl)-4-phenyl-1-tosyl-1,2,3,4-tetrahydro-6H-benzo[c][1,5]oxazocin-6-one, 6a $49.4 \mathrm{mg}, 99 \%$; white solid (starting from $42.1 \mathrm{mg}$ of 5a); $\mathrm{MP} 183-184^{\circ} \mathrm{C} ;[\alpha]_{D}^{25}=+4.9(c 1.0, \mathrm{MeOH}$, $81 \%$ ee); ${ }^{1} \mathrm{H}$ NMR $\left(\mathrm{CDCl}_{3}, 400 \mathrm{MHz}\right): \delta 7.60-7.21(\mathrm{~m}, 13 \mathrm{H}), 3.87-3.73(\mathrm{~m}, 2 \mathrm{H}), 3.77\left(\mathrm{dd}, J_{l}=12.0\right.$ $\left.\mathrm{Hz}, J_{2}=72.0 \mathrm{~Hz}, 2 \mathrm{H}\right), 2.64-2.58(\mathrm{~m}, 1 \mathrm{H}), 2.46(\mathrm{~s}, 3 \mathrm{H}), 2.16-2.10(\mathrm{~m}, 1 \mathrm{H}) ;{ }^{13} \mathrm{C} \mathrm{NMR}\left(\mathrm{CDCl}_{3}, 100\right.$ $\mathrm{MHz}): \delta 167.4,144.0,140.0,136.3,135.4,134.9,132.8,131.6,130.7,129.7,128.8,128.6,127.6$, 125.6, 84.7, 45.4,42.2, 32.0, 21.7; HRMS (TOF) $\mathrm{m} / \mathrm{z}$ : $[\mathrm{M}+\mathrm{H}]^{+}$Calcd for $\mathrm{C}_{24} \mathrm{H}_{23} \mathrm{BrNO}_{4} \mathrm{~S} 500.6147$; found 500.6146; HPLC (Daicel Chiralcel OD-H, $i-\mathrm{PrOH} / \mathrm{Hexane}=35 / 65,1.0 \mathrm{~mL} / \mathrm{min}, 210 \mathrm{~nm}$ ) $\mathrm{t}_{1}=$ $15.8 \mathrm{~min}$ (major), $\mathrm{t}_{2}=20.1 \mathrm{~min}$ (minor).

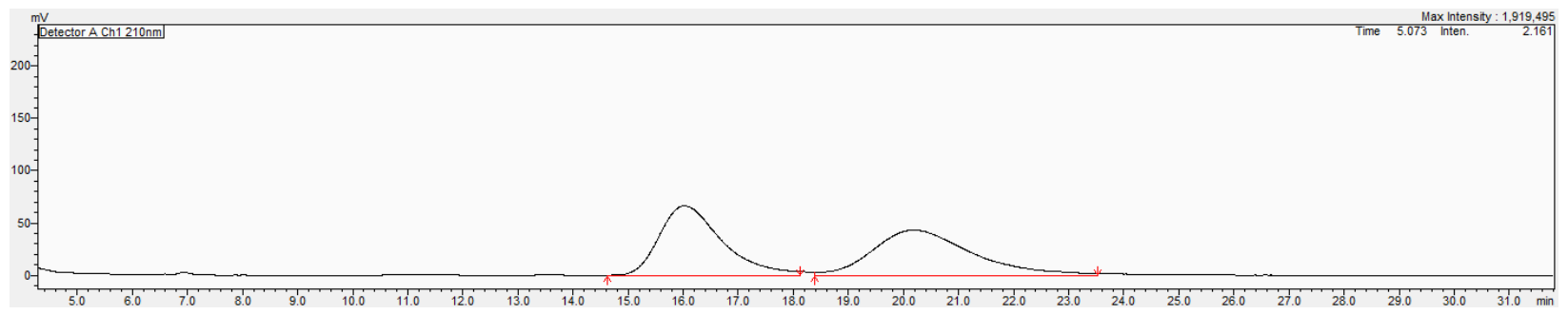

\begin{tabular}{|l|r|r|r|r|r|}
\hline Peak\# & Ret. Time & \multicolumn{1}{c|}{ Area } & Height & Mark & \multicolumn{1}{|c|}{ Conc. } \\
\hline 1 & 16.013 & 5320210 & 66637 & $V$ & 49.165 \\
\hline 2 & 20.194 & 5500860 & 43538 & $V$ & 50.835 \\
\hline Total & & 10821070 & 110175 & & 100.000 \\
\hline
\end{tabular}




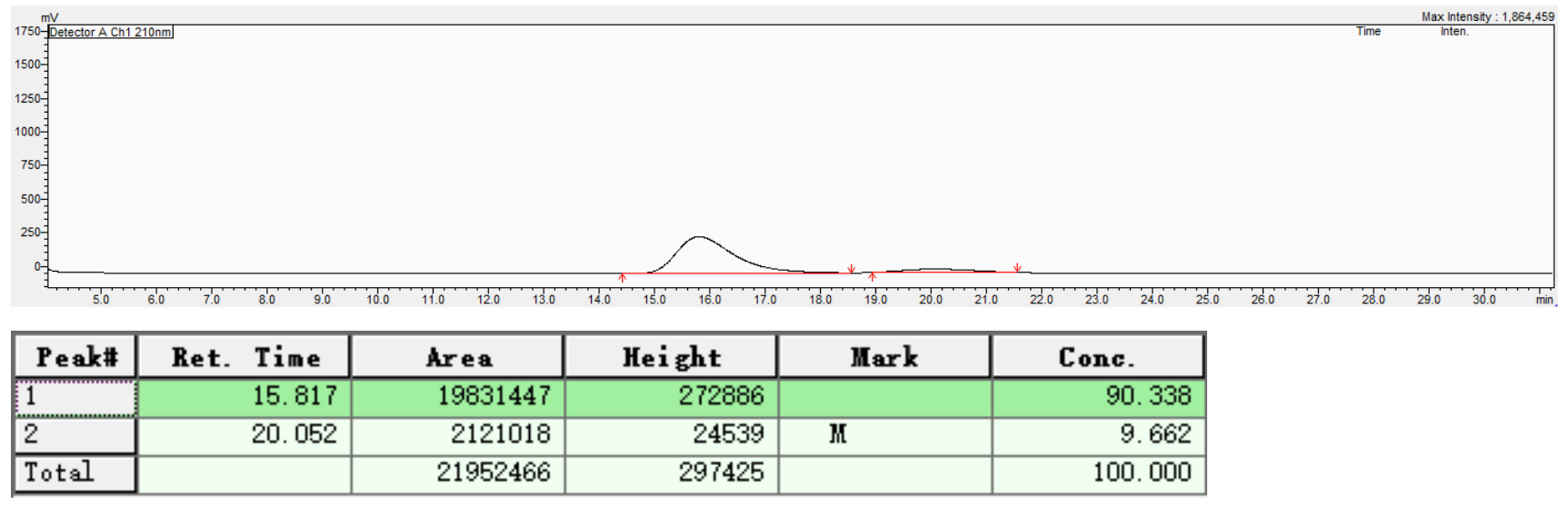<smiles>O=C1OC(CBr)(c2ccccc2)COc2ccccc21</smiles>

(R)-3-(bromomethyl)-3-phenyl-2,3-dihydro-5H-benzo[e][1,4]dioxepin-5-one, $6 \mathrm{~b}$ $20.9 \mathrm{mg}$, 63\%; colorless oil (starting from $25.4 \mathrm{mg}$ of $\mathbf{5 b}) ;[\alpha]_{D}^{25}=+7.7\left(c 1.0, \mathrm{MeOH}, 32 \%\right.$ ee); ${ }^{1} \mathrm{H}$ NMR $\left(\mathrm{CDCl}_{3}, 400 \mathrm{MHz}\right): \delta 8.12-6.80(\mathrm{~m}, 9 \mathrm{H}), 5.07\left(\mathrm{dd}, J_{1}=12.0 \mathrm{~Hz}, J_{2}=104.0 \mathrm{~Hz}, 2 \mathrm{H}\right), 3.91(\mathrm{dd}$, $\left.J_{I}=12.0 \mathrm{~Hz}, J_{2}=40.0 \mathrm{~Hz}, 2 \mathrm{H}\right) ;{ }^{13} \mathrm{C} \mathrm{NMR}\left(\mathrm{CDCl}_{3}, 100 \mathrm{MHz}\right): \delta 165.8,156.3,136.5,135.2,134.9$, 128.8, 128.7, 126.0, 121.6, 119.5, 116.6, 87.9, 74.3, 38.4; HRMS (TOF) m/z: $[\mathrm{M}+\mathrm{H}]^{+}$Calcd for $\mathrm{C}_{16} \mathrm{H}_{14} \mathrm{BrO}_{3}$ 333.1651; found 333.1652; HPLC (Daicel Chiralcel IC, $i$-PrOH/Hexane $=10 / 90,1.0$ $\mathrm{mL} / \mathrm{min}, 210 \mathrm{~nm}$ ) $\mathrm{t}_{1}=13.4 \mathrm{~min}$ (major), $\mathrm{t}_{2}=15.1 \mathrm{~min}$ (minor).

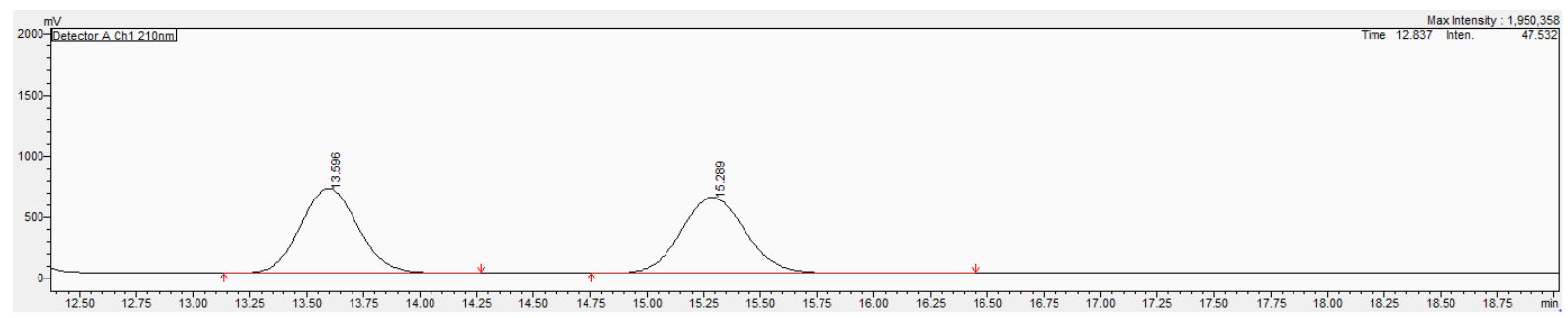

\begin{tabular}{|l|r|r|r|r|r|}
\hline Peak\# & Ret. Time & \multicolumn{1}{c|}{ Area } & Hei ght & \multicolumn{1}{c|}{ Mark } & \multicolumn{1}{c|}{ Conc. } \\
\hline 1 & 13.596 & 12132730 & 691642 & & 49.921 \\
\hline 2 & 15.289 & 12171052 & 617218 & & 50.079 \\
\hline Total & & 24303782 & 1308860 & & 100.000 \\
\hline
\end{tabular}

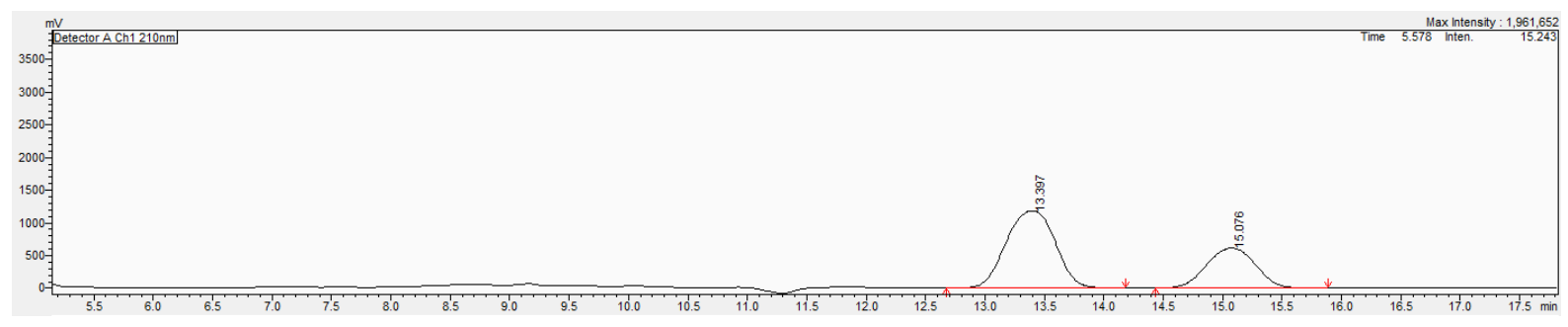

\begin{tabular}{|l|r|r|r|r|r|}
\hline Peak\# & Ret. Time & Area & Hei ght & Mark & \multicolumn{1}{|c|}{ Conc. } \\
\hline 1 & 13.397 & 33776611 & 1179501 & M & 66.002 \\
\hline 2 & 15.076 & 17398328 & 610535 & M & 33.998 \\
\hline Total & & 51174939 & 1790035 & & 100.000 \\
\hline
\end{tabular}




\section{Asymmetric Chlorolactonization of 7.}

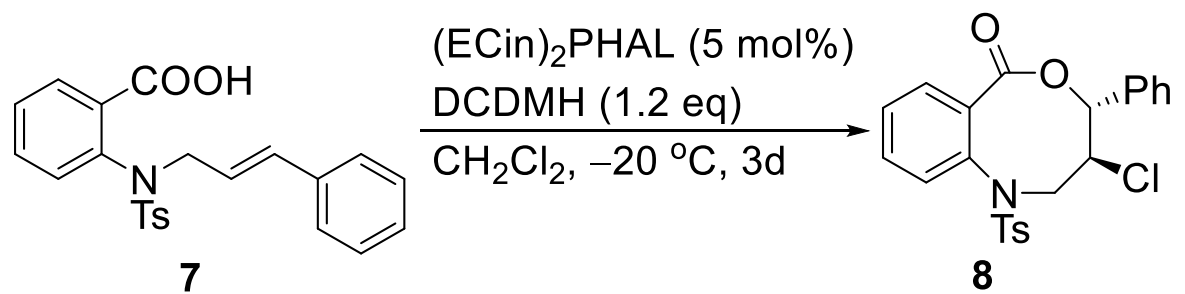

To a solution of 7 (40.7 mg, $0.1 \mathrm{mmol}, 1.0$ equiv) and (ECin) $)_{2}$ PHAL (3.6 mg, $0.005 \mathrm{mmol}, 0.05$ equiv) in $\mathrm{CH}_{2} \mathrm{Cl}_{2}(8.0 \mathrm{~mL})$ was added DCDMH portionwise $(23.6 \mathrm{mg}, 1.2 \mathrm{mmol}, 1.2$ equiv) at -20 ${ }^{\circ} \mathrm{C}$. The mixture was then stirred for 3 days at $-20{ }^{\circ} \mathrm{C}$. The reaction was quenched with saturated $\mathrm{Na}_{2} \mathrm{SO}_{3}(1.0 \mathrm{~mL})$ at room temperature. The solution was diluted with water $(3.0 \mathrm{~mL})$ and extrated with EtOAc, dried over $\mathrm{MgSO}_{4}$ and concentrated in vacuo. The residue was purified by flash column chromatography (hexane/EtOAc) to yield $8\{(3 S, 4 R)-3-c h l o r o-4-p h e n y l-1-t o s y l-1,2,3,4-$

tetrahydro-6H-benzo[c][1,5]oxazocin-6-one $\}$ as white solid; $41.4 \mathrm{mg}, 94 \%$; $\mathrm{MP} 171-172{ }^{\circ} \mathrm{C} ;[\alpha]_{D}^{25}=$ -4.1 (c 1.0, $\mathrm{MeOH}, 91 \%$ ee); ${ }^{1} \mathrm{H}$ NMR $\left(\mathrm{CDCl}_{3}, 400 \mathrm{MHz}\right): \delta 7.72-7.13(\mathrm{~m}, 13 \mathrm{H}), 5.15(\mathrm{~d}, J=8.0$ $\mathrm{Hz}, 1 \mathrm{H}), 4.81-4.60(\mathrm{~m}, 2 \mathrm{H}), 3.45-3.38(\mathrm{~m}, 1 \mathrm{H}), 2.47$ (s, 3H); ${ }^{13} \mathrm{C} \mathrm{NMR}\left(\mathrm{CDCl}_{3}, 100 \mathrm{MHz}\right): \delta 167.1$, 144.4, 138.2, 137.1, 136.3, 133.5, 132.6, 131.1, 130.0, 129.9, 129.4, 129.2, 128.4, 127.6,84.1, 57.6, 56.3, 21.7; HRMS (TOF) $\mathrm{m} / \mathrm{z}:[\mathrm{M}+\mathrm{H}]^{+}$Calcd for $\mathrm{C}_{23} \mathrm{H}_{21} \mathrm{ClNO}_{4} \mathrm{~S}$ 442.5077; found 442.5076; HPLC (Daicel Chiralcel OD-H, $i$-PrOH/Hexane $=35 / 65,1.0 \mathrm{~mL} / \mathrm{min}, 210 \mathrm{~nm}) \mathrm{t}_{1}=9.0 \mathrm{~min}($ major$), \mathrm{t}_{2}=$ $12.4 \min$ (minor).

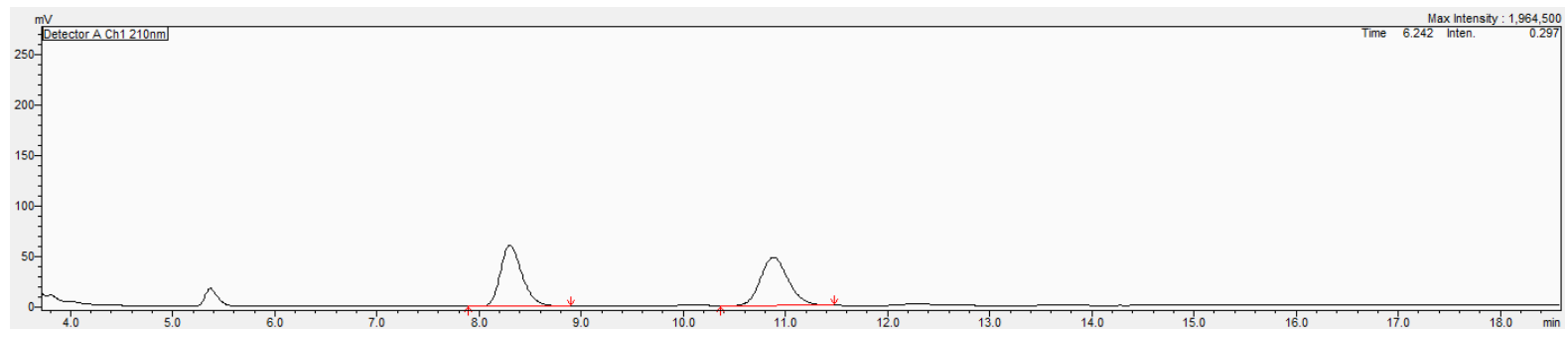

\begin{tabular}{|l|r|r|r|r|r|}
\hline Peak\# & Ret. Time & Area & Hei ght & Mark & \multicolumn{1}{c|}{ Conc. } \\
\hline 1 & 8.303 & 894539 & 60257 & M & 49.814 \\
\hline 2 & 10.883 & 901219 & 47864 & M & 50.186 \\
\hline Total & & 1795758 & 108121 & & 100.000 \\
\hline
\end{tabular}

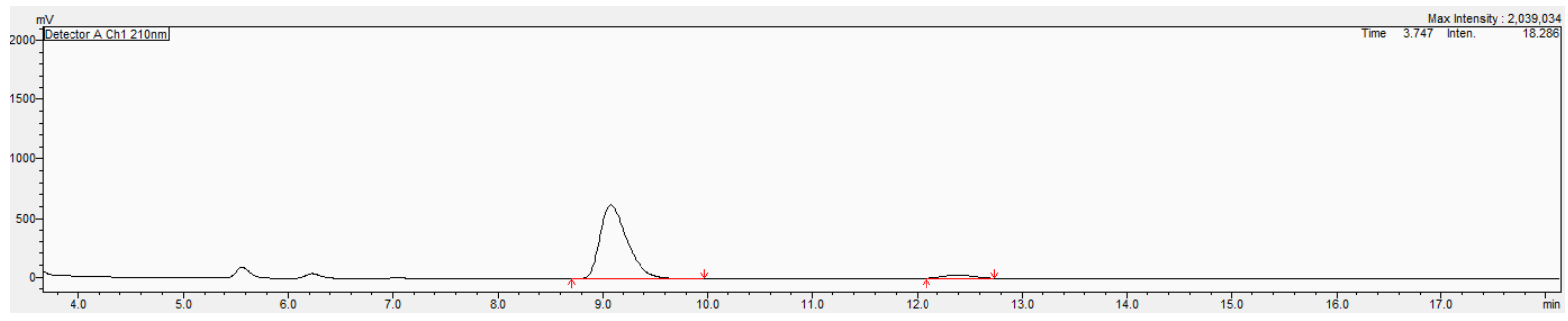

\begin{tabular}{|l|r|r|r|r|r|}
\hline Peak\# & Ret. Time & \multicolumn{1}{c|}{ Area } & Hei ght & \multicolumn{1}{c|}{ Mark } & \multicolumn{1}{c|}{ Conc. } \\
\hline 1 & 9.078 & 11245283 & 625529 & V & 95.307 \\
\hline 2 & 12.388 & 553751 & 27830 & M & 4.693 \\
\hline Total & & 11799034 & 653359 & & 100.000 \\
\hline
\end{tabular}


<smiles>O=C1O[C@H](c2ccccc2)[C@H](Cl)CN([As])c2ccccc21</smiles>

ent-8

$41.9 \mathrm{mg}, 95 \% ;[\alpha]_{D}^{25}=+5.9(c 1.0, \mathrm{MeOH}, 80 \%$ ee); HPLC (Daicel Chiralcel AD-H, $i$-PrOH/Hexane $=35 / 65,1.0 \mathrm{~mL} / \mathrm{min}, 210 \mathrm{~nm}$ ) $\mathrm{t}_{1}=8.3 \mathrm{~min}$ (minor), $\mathrm{t}_{2}=11.9 \mathrm{~min}$ (major).

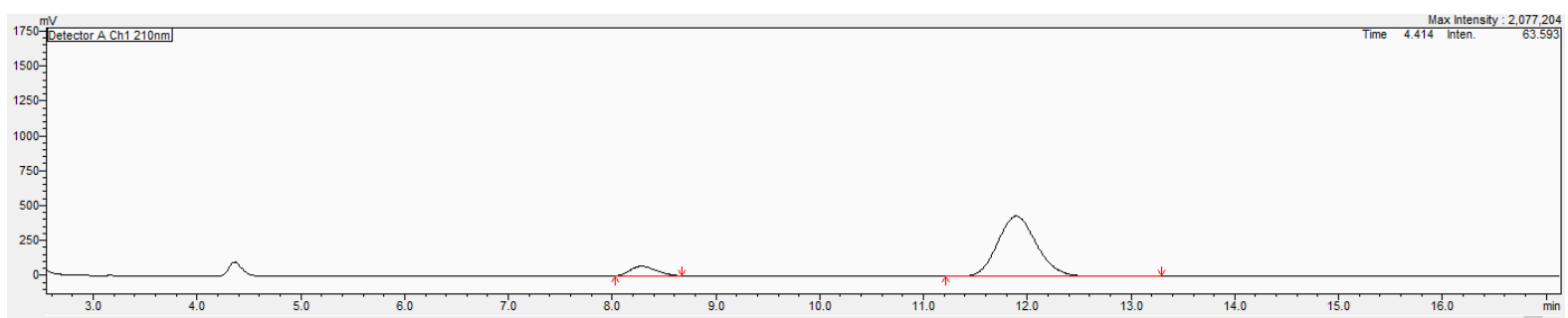

\begin{tabular}{|l|r|r|r|r|r|}
\hline Peak\# & Ret. Time & Area & Hei ght & Mark & \multicolumn{1}{|c|}{ Conc. } \\
\hline 1 & 8.283 & 1219110 & 68521 & M & 9.734 \\
\hline 2 & 11.894 & 11305077 & 433244 & M & 90.266 \\
\hline Total & & 12524187 & 501765 & & 100.000 \\
\hline
\end{tabular}

\section{Synthesis of 9}<smiles>O=C1O[C@](CBr)(c2ccccc2)CNc2ccccc21</smiles>

2d, $98 \%$ ee<smiles>O=C1O[C@](Cc2ccccc2)(c2ccccc2)CNc2ccccc21</smiles>

9, $98 \%$ ee

To a solution of $2 \mathbf{d}(51.0 \mathrm{mg}, 0.1 \mathrm{mmol}, 1.0$ equiv) in THF $(2.0 \mathrm{~mL})$, was added sodium thiophenolate $(66.0 \mathrm{mg}, 0.5 \mathrm{mmol}, 5.0$ equiv) at room temperature. The mixture was then stirred for $4 \mathrm{~h}$ at room temperature. Then water and EtOAc were added. The organic layer was washed with water, and dried over magnesium sulfate. Concentration of the organic layer offered the crude product that was further purified by flash column chromatography (hexane/EtOAc) to give the corresponding 9 as yellow oil, $30.7 \mathrm{mg}, 85 \% ;[\alpha]_{D}^{25}=+20.5(c 1.0, \mathrm{MeOH}, 98 \%$ ee $) ;{ }^{1} \mathrm{H} \mathrm{NMR}\left(\mathrm{CDCl}_{3}\right.$, $400 \mathrm{MHz}): \delta$ 7.99-6.64 (m, 14H), 3.75-3.56 (m, 4H); ${ }^{13} \mathrm{C} \mathrm{NMR}\left(\mathrm{CDCl}_{3}, 100 \mathrm{MHz}\right): \delta 173.5,151.7$, 142.8, 136.1, 135.5, 132.6,130.2, 129.1, 128.9, 127.8, 125.7,125.5, 115.2, 111.7, 109.4, 76.1, 52.7, 45.8; HRMS (TOF) m/z: [M+H] ${ }^{+}$Calcd for $\mathrm{C}_{22} \mathrm{H}_{20} \mathrm{NO}_{2} \mathrm{~S}$ 362.5549; found 362.5548; HPLC (Daicel Chiralcel IC, $i$-PrOH/Hexane $=10 / 90,1.0 \mathrm{~mL} / \mathrm{min}, 254 \mathrm{~nm}$ ) $\mathrm{t}_{1}=10.0 \mathrm{~min}$ (major), $\mathrm{t}_{2}=13.0 \mathrm{~min}$ (minor). 


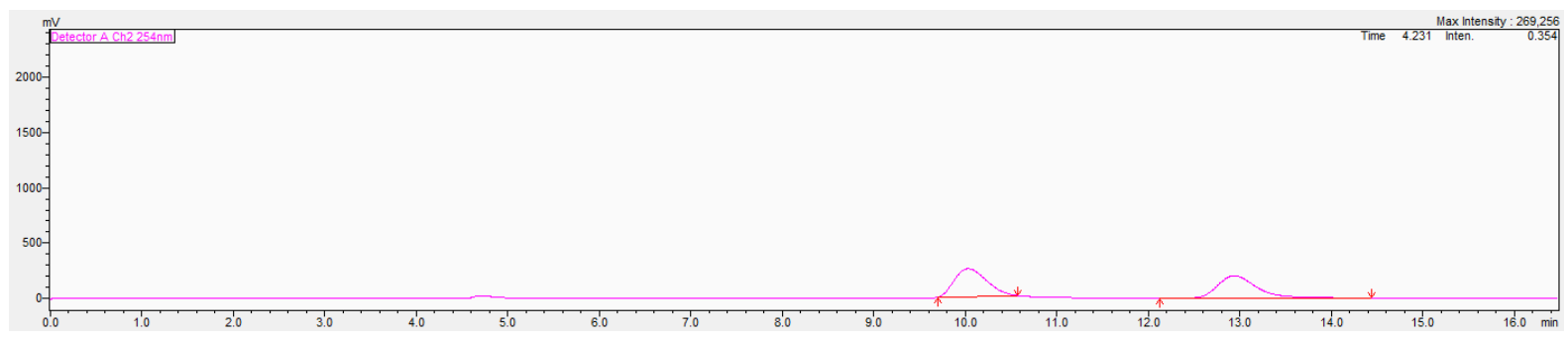

\begin{tabular}{|l|r|r|r|r|r|}
\hline Peak\# & Ret. Time & Area & Hei ght & Mark & \multicolumn{1}{c|}{ Conc. } \\
\hline 1 & 10.029 & 6147131 & 252650 & M & 50.661 \\
\hline 2 & 12.937 & 5986653 & 202249 & $M$ & 49.339 \\
\hline Total & & 12133785 & 454899 & & 100.000 \\
\hline
\end{tabular}

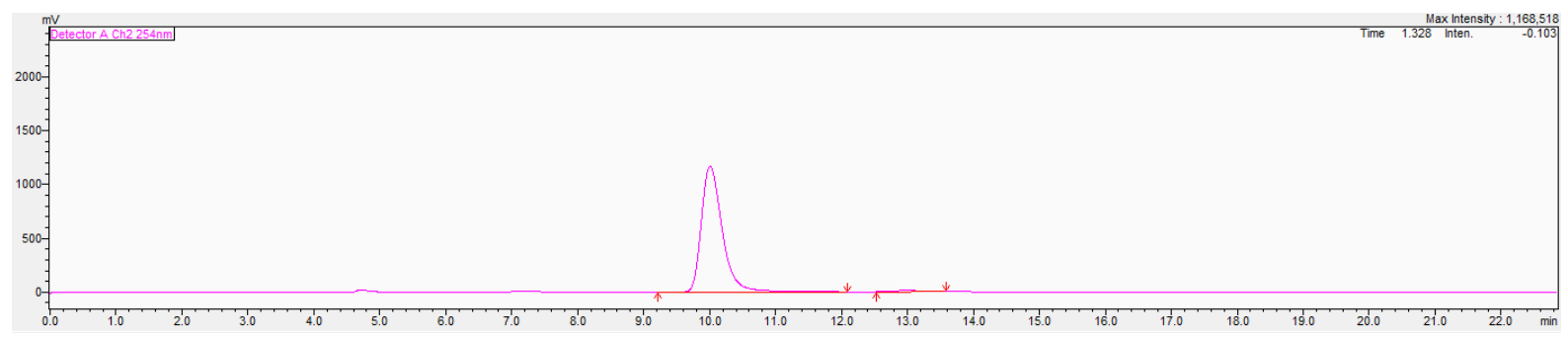

\begin{tabular}{|l|r|r|r|r|r|}
\hline Peak\# & Ret. Time & \multicolumn{1}{c|}{ Area } & \multicolumn{1}{c|}{ Hei ght } & \multicolumn{1}{c|}{ Mark } & \multicolumn{1}{c|}{ Conc. } \\
\hline 1 & 10.006 & 25908257 & 1169120 & $M$ & 98.730 \\
\hline 2 & 12.984 & 333230 & 11904 & $M$ & 1.270 \\
\hline Total & & 26241488 & 1181024 & & 100.000 \\
\hline
\end{tabular}<smiles>CS(=O)(=O)N1C[C@](CBr)(c2ccccc2)OC(=O)c2ccccc21</smiles>

ent-2d, $98 \%$ ee<smiles>O=C1O[C@](CSc2ccccc2)(c2ccccc2)CNc2ccccc21</smiles>

ent-9, $97 \%$ ee

$30.7 \mathrm{mg}$, yellow oil, 85\%; $[\alpha]_{D}^{25}=-20.6$ (c 1.0, MeOH, 97\% ee); HPLC (Daicel Chiralcel IC, $i$-PrOH/Hexane $=10 / 90,1.0 \mathrm{~mL} / \min , 254 \mathrm{~nm}$ ) $\mathrm{t}_{1}=10.4 \min$ (minor), $\mathrm{t}_{2}=13.0$ min (major).

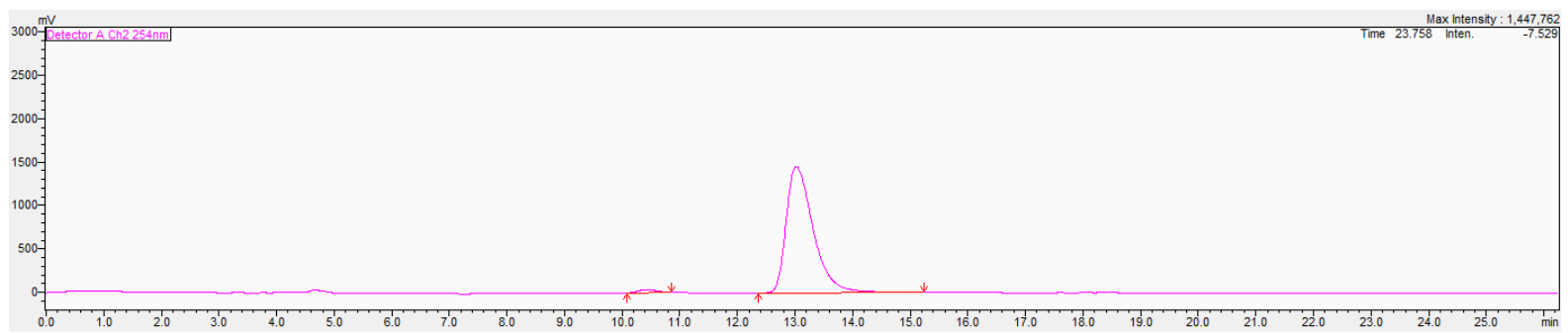

\begin{tabular}{|l|r|r|r|r|r|}
\hline \multicolumn{1}{|c|}{ Peak\# } & Ret. Time & \multicolumn{1}{c|}{ Area } & \multicolumn{1}{c|}{ Hei ght } & \multicolumn{1}{c|}{ Mark } & \multicolumn{1}{c|}{ Conc. } \\
\hline 1 & 10.428 & 816453 & 35725 & M & 1.692 \\
\hline 2 & 13.026 & 47443416 & 1453651 & & 98.308 \\
\hline Total & & 48259870 & 1489376 & & 100.000 \\
\hline
\end{tabular}




\section{X-ray of 1a-(DHQ) ${ }_{2}$ PHAL}

Sample preparation for crystal growth: Compound 1a $(40.7 \mathrm{mg})$ and (DHQ) ${ }_{2} \mathrm{PHAL}(39.0 \mathrm{mg})$ were dissolved in EtOAc $(7 \mathrm{~mL})$, while slow evaporation of solvent at room temperature yellow crystals were grown.

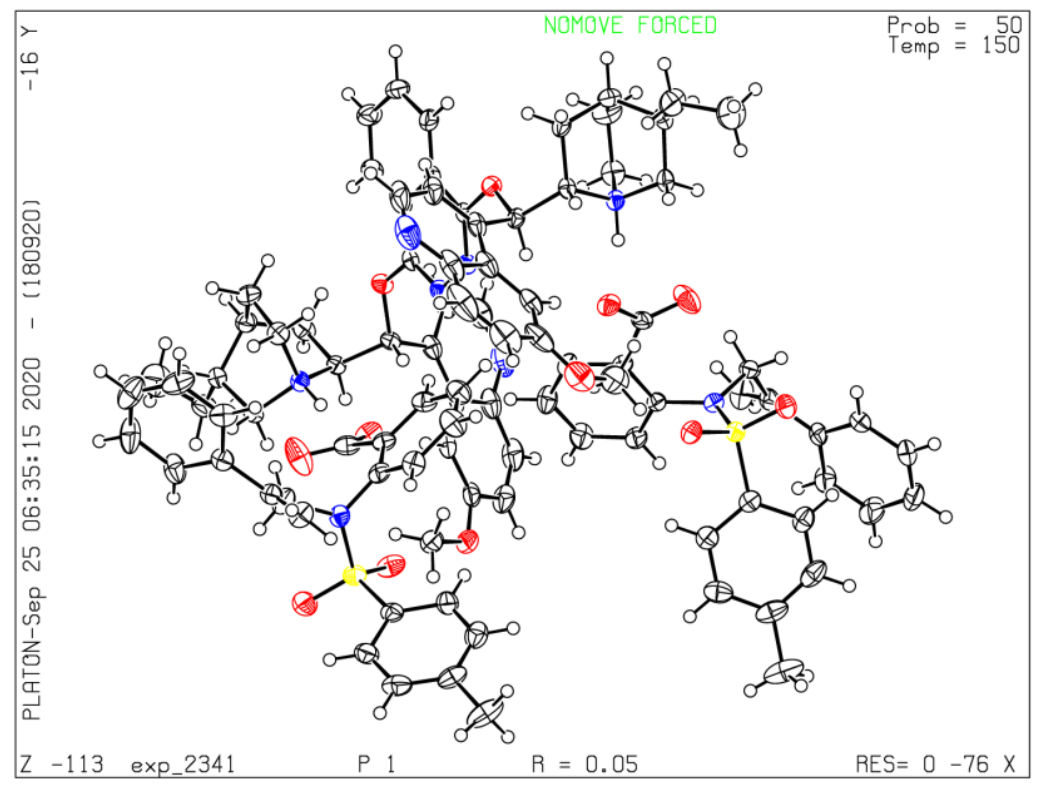

Thermal ellipsoids are shown at 50\% probability.

\section{Different views of 1a-(DHQ) $)_{2}$ PHAL:}
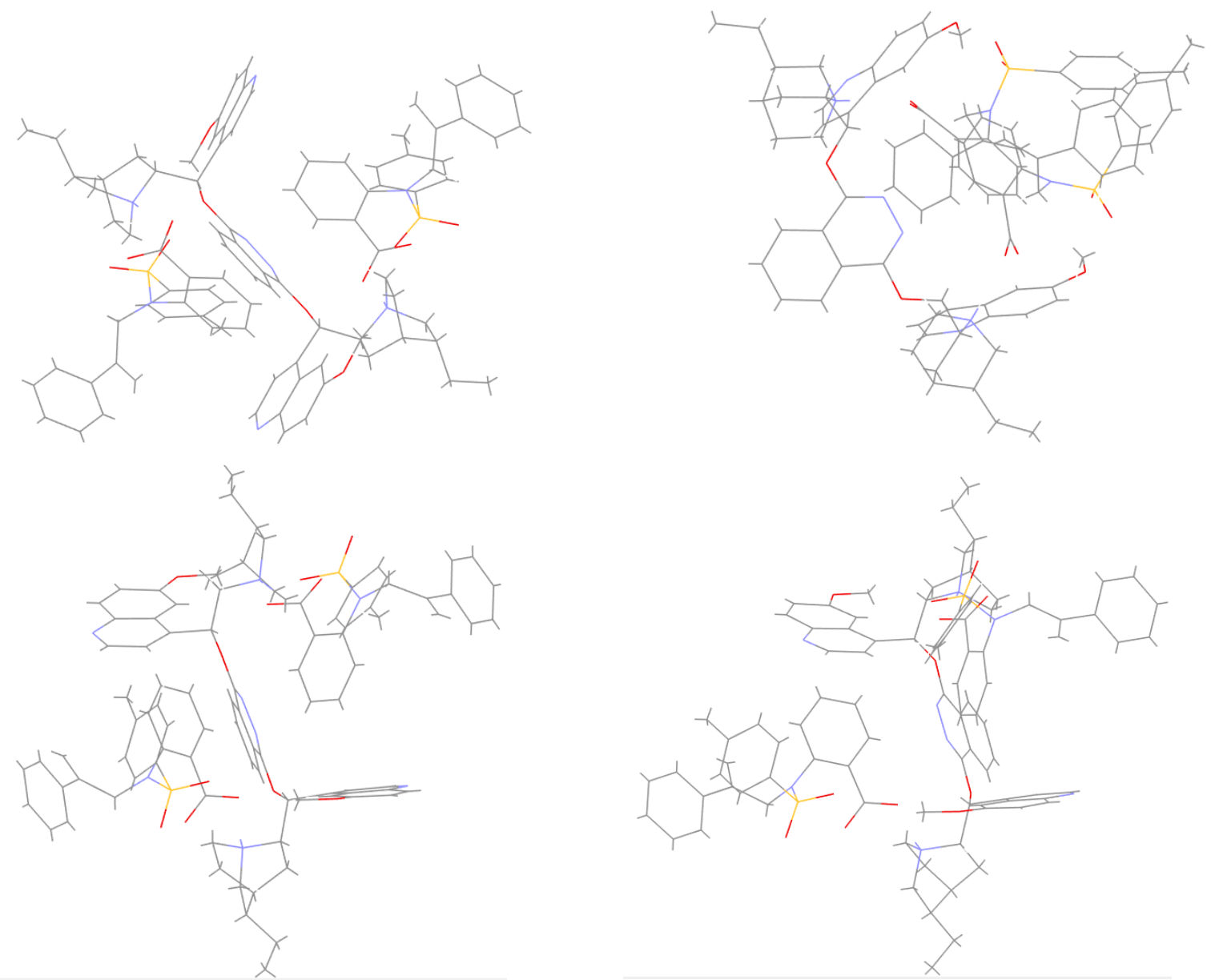


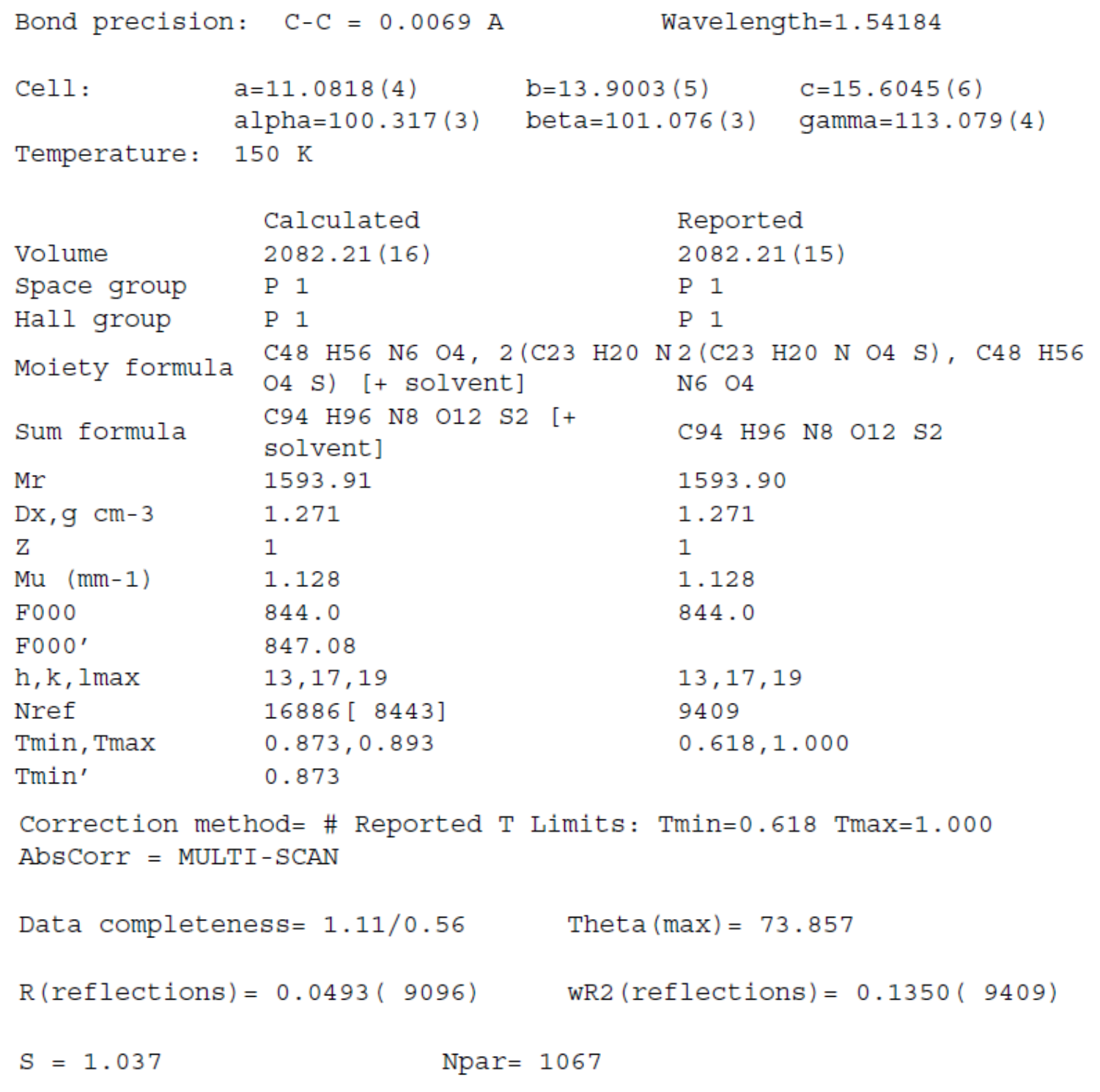




\section{X-ray of 2a}

Sample preparation for crystal growth: Compound 2a $(40 \mathrm{mg})$ was dissolved in EtOAc/Hexane (v/v = 3 $\mathrm{mL} / 1 \mathrm{~mL}$ ), while slow evaporation of solvent at room temperature white crystals were grown.

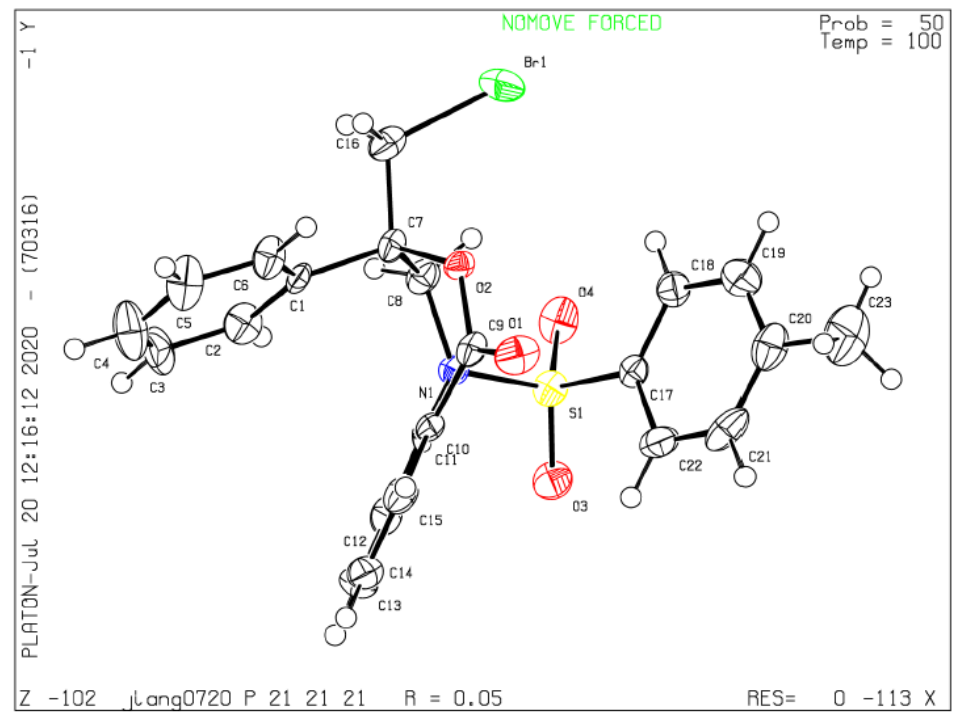

Thermal ellipsoids are shown at 50\% probability.

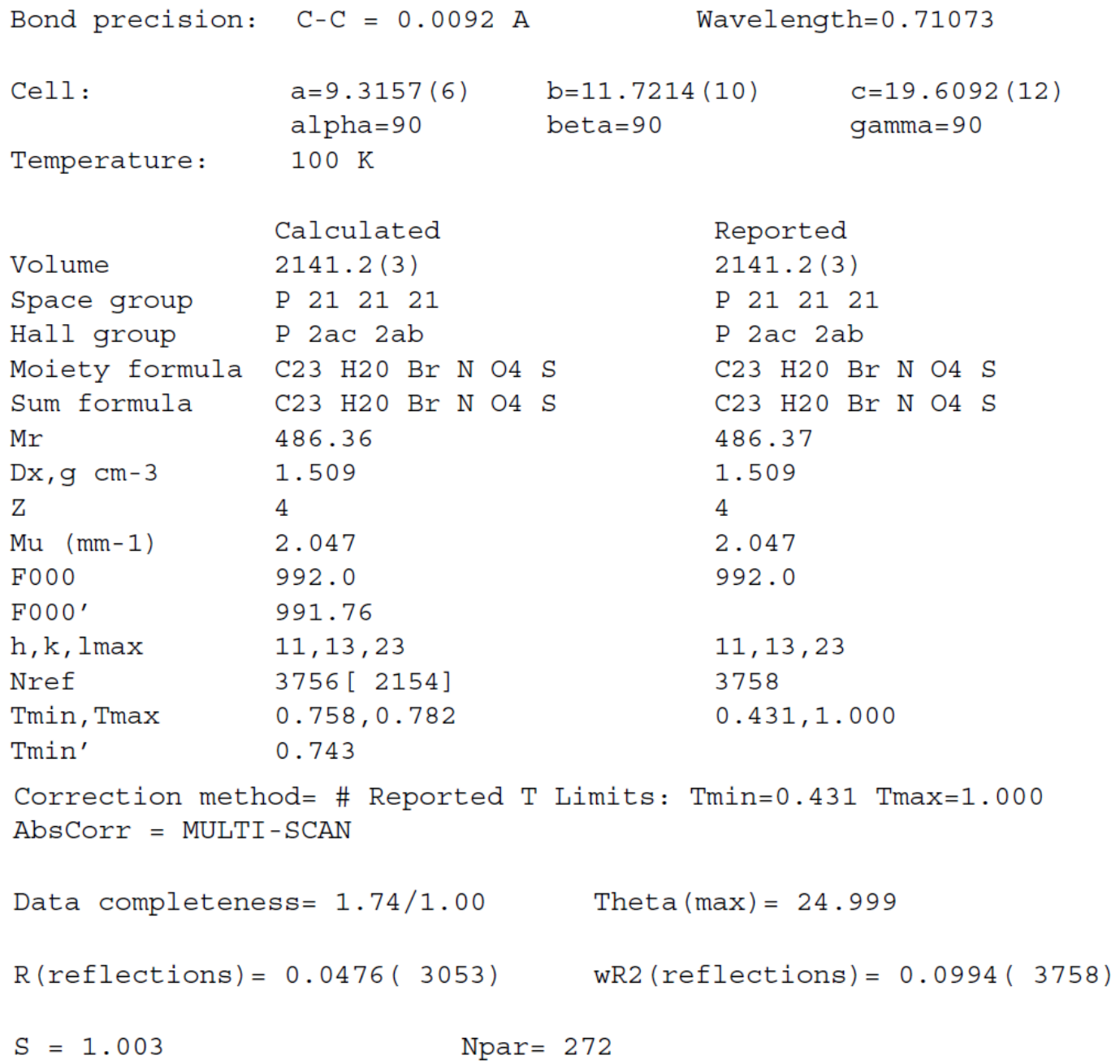




\section{X-ray of $4 a$}

Sample preparation for crystal growth: Compound $\mathbf{4 a}(40 \mathrm{mg})$ was dissolved in EtOAc/Hexane (v/v $=3$ $\mathrm{mL} / 1 \mathrm{~mL}$ ), while slow evaporation of solvent at room temperature white crystals were grown.

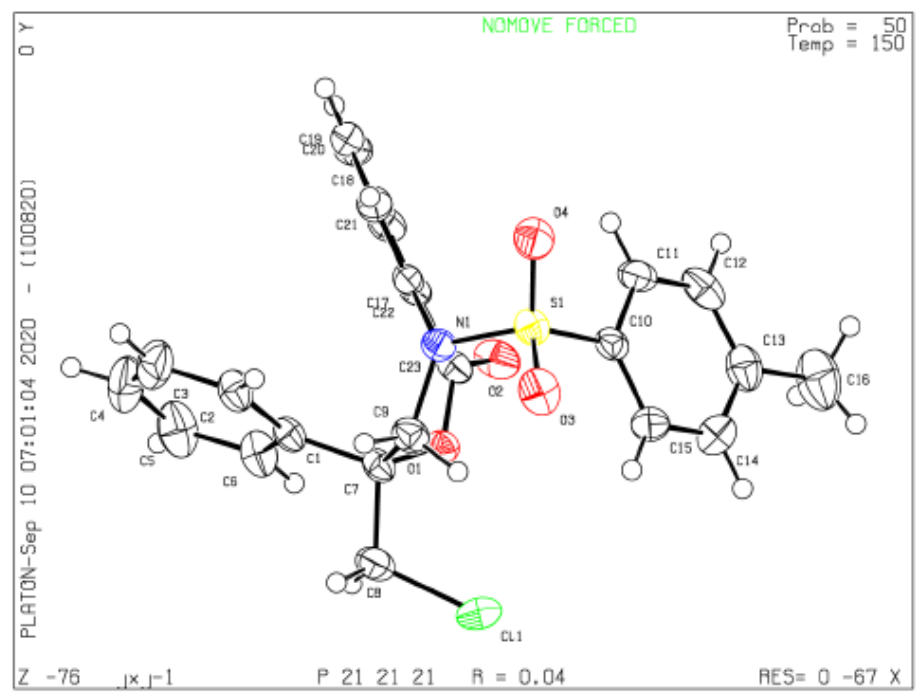

Thermal ellipsoids are shown at 50\% probability.

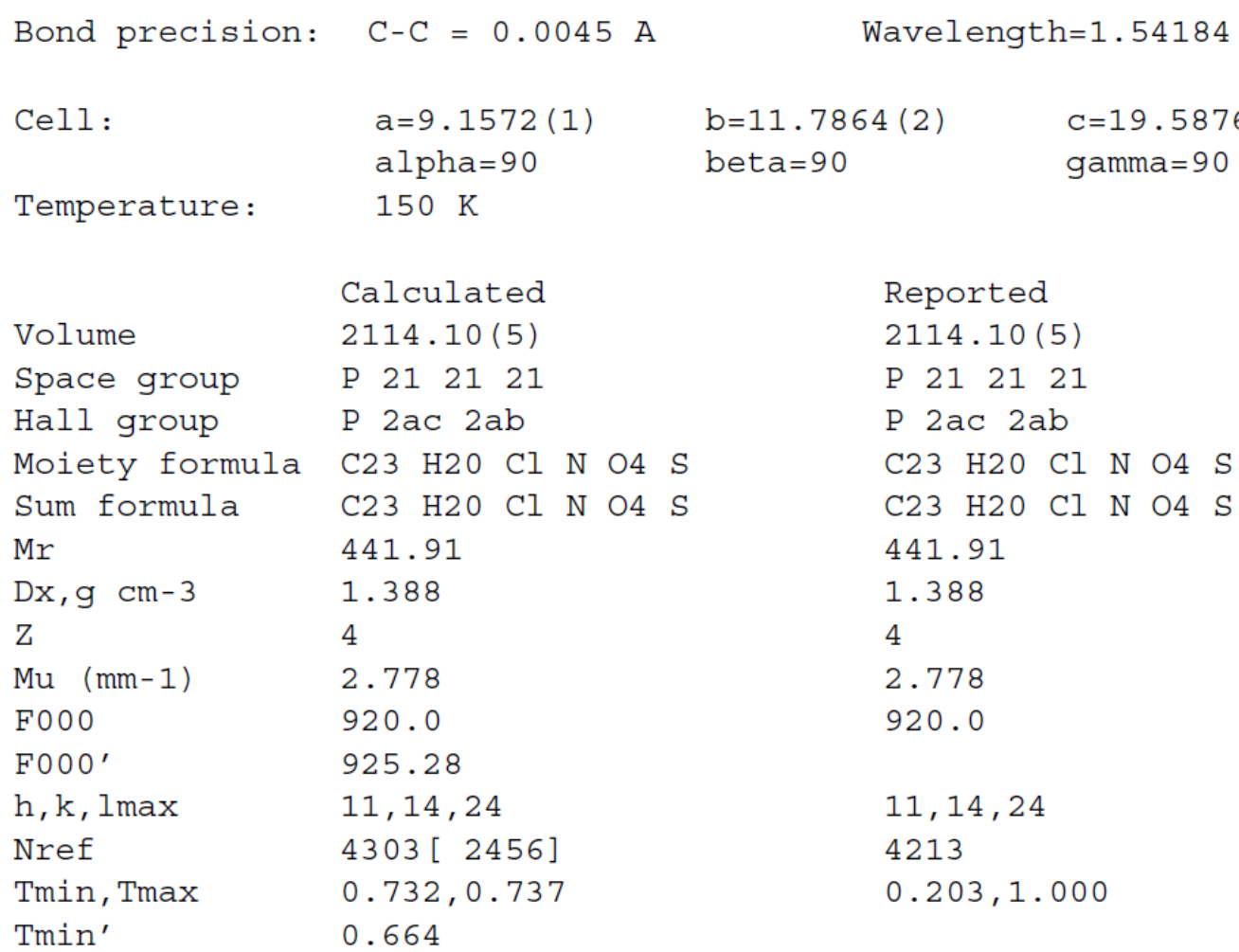

Correction method= \# Reported T Limits: Tmin=0.203 Tmax=1.000 AbsCorr = MULTI - SCAN

Data completeness $=1.72 / 0.98 \quad$ Theta $(\max )=73.954$

$R($ reflections $)=0.0368(4064) \quad$ wR2 (reflections $)=0.0989(4213)$

$S=1.033 \quad$ Npar $=273$ 


\section{X-ray of ent-8}

Sample preparation for crystal growth: Compound ent-8 $(40 \mathrm{mg})$ was dissolved in EtOAc/Hexane (v/v $=3$ $\mathrm{mL} / 1 \mathrm{~mL}$ ), while slow evaporation of solvent at room temperature white crystals were grown.

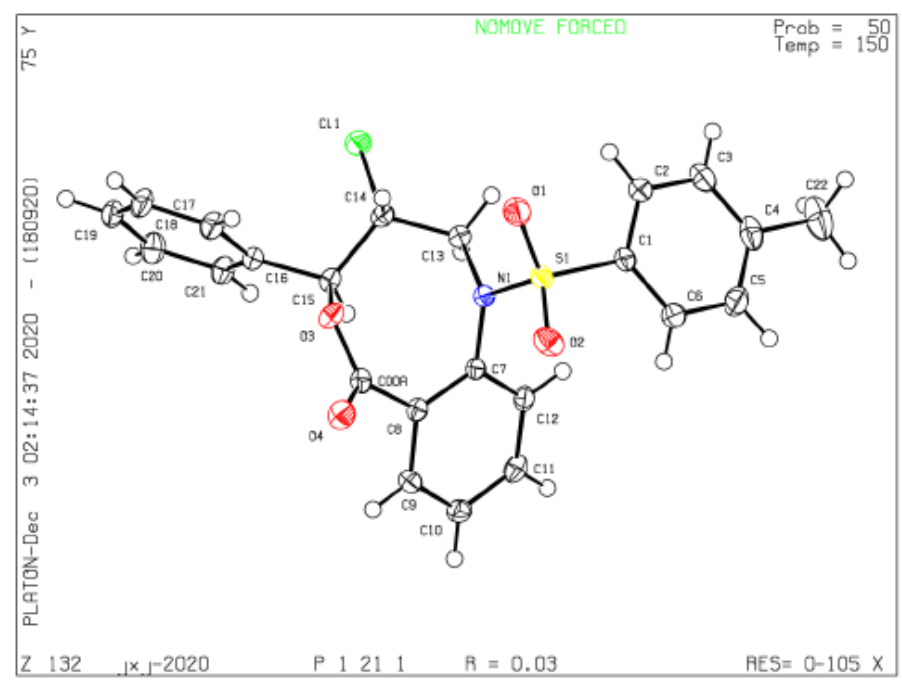

Thermal ellipsoids are shown at 50\% probability.

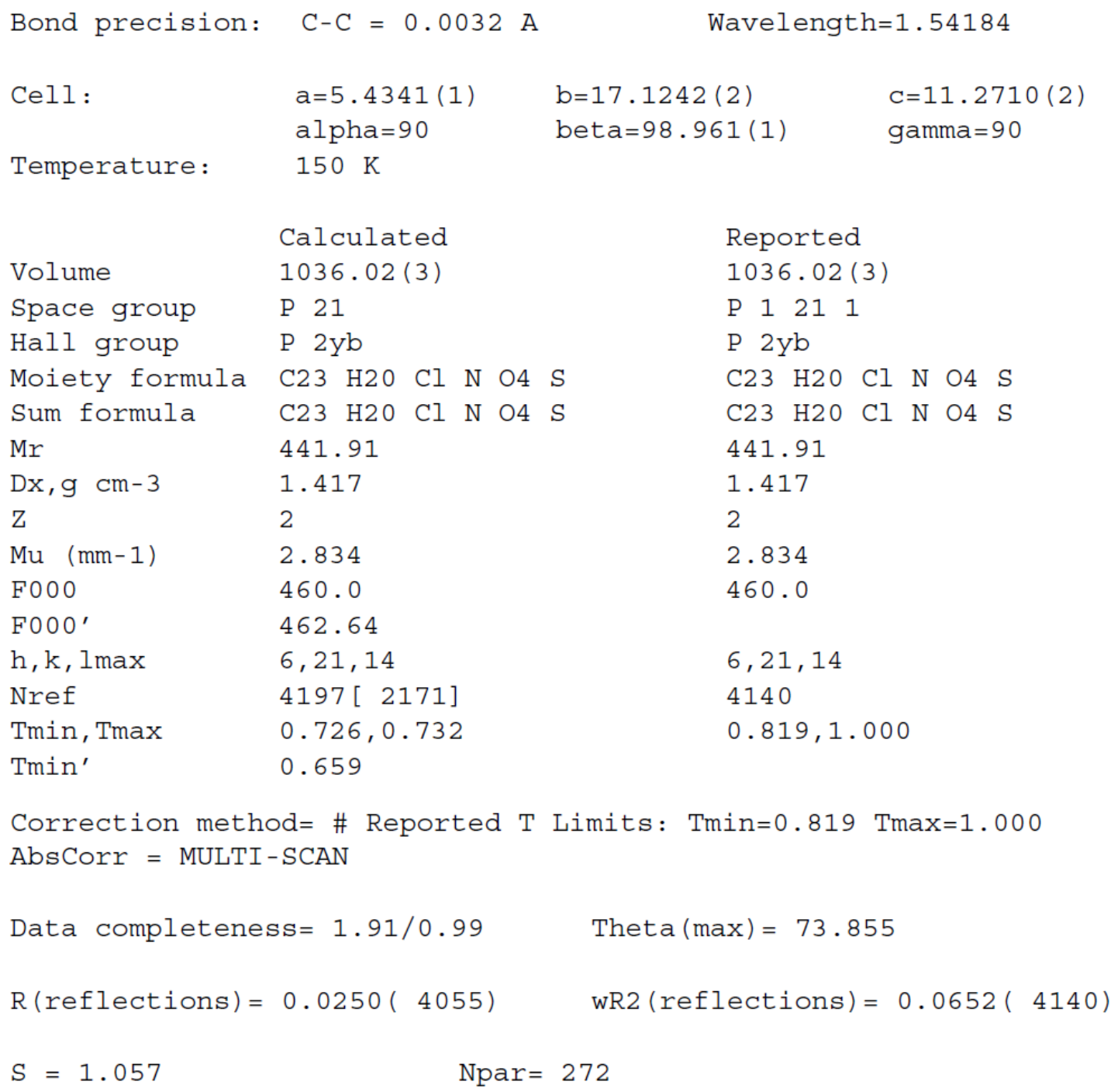




\section{${ }^{1} \mathrm{H} /{ }^{13} \mathrm{C}$ NMR}

gm. 2020. cinchonine配体. 1. fid

gm. 2020. 12. 29. b

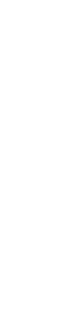$$
\left.\iiint|| \mid \int\right\}
$$$$
\iint
$$

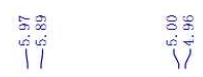

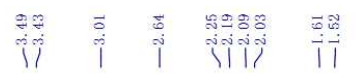
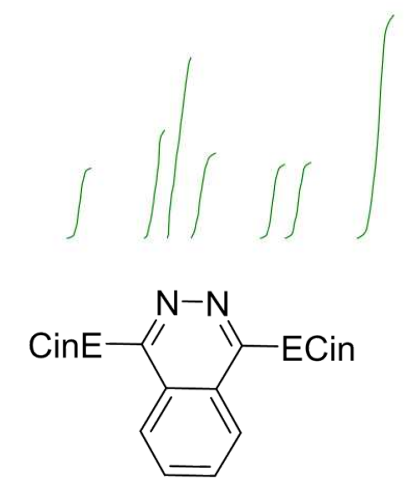

(Cin) $)_{2} \mathrm{PHAL}$

$400 \mathrm{MHz}, \mathrm{CDCl}_{3}$
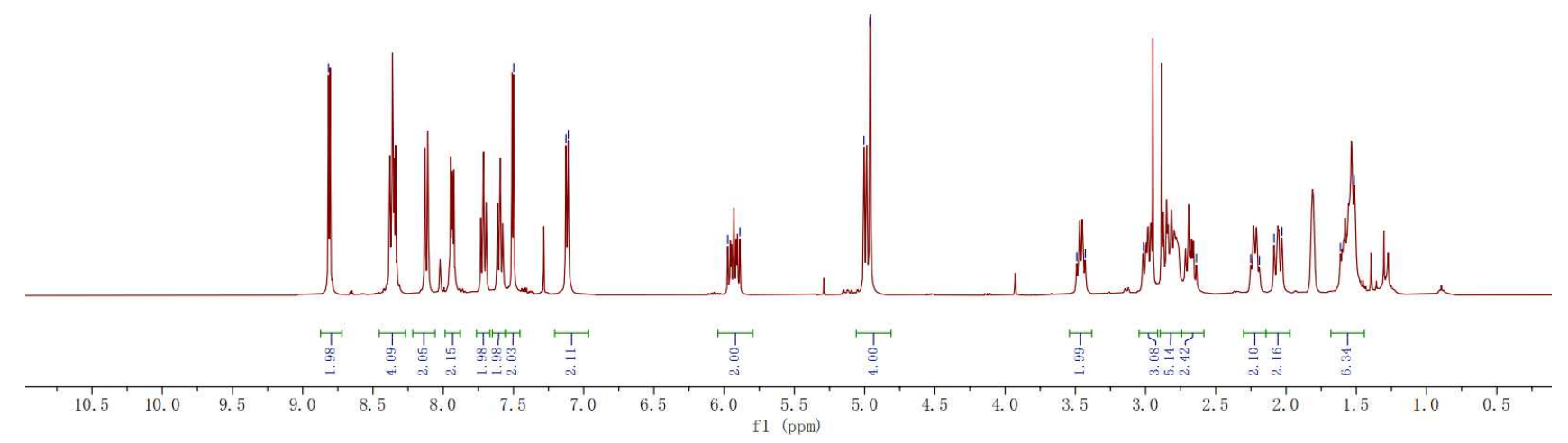

gm. 2020. cinchonine配体. 2. fid gm. 2020.12. 29. b

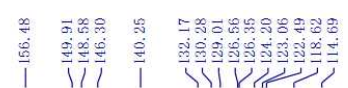

$\begin{array}{lll}1 & 1 & 1\end{array}$

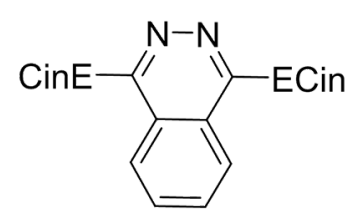

(Cin) $)_{2}$ PHAL

$100 \mathrm{MHz}, \mathrm{CDCl}_{3}$

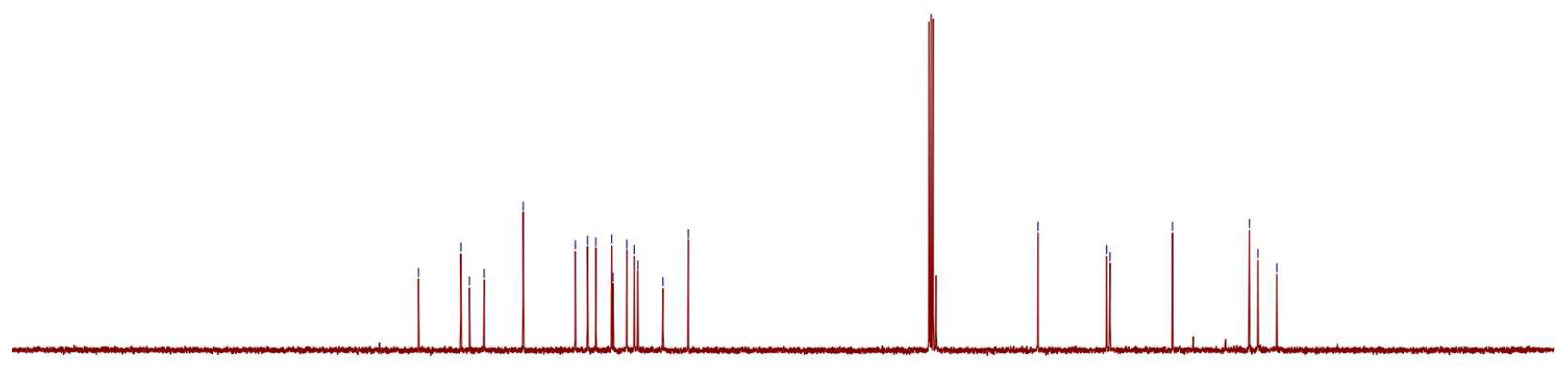

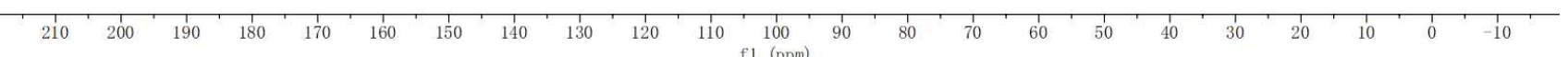



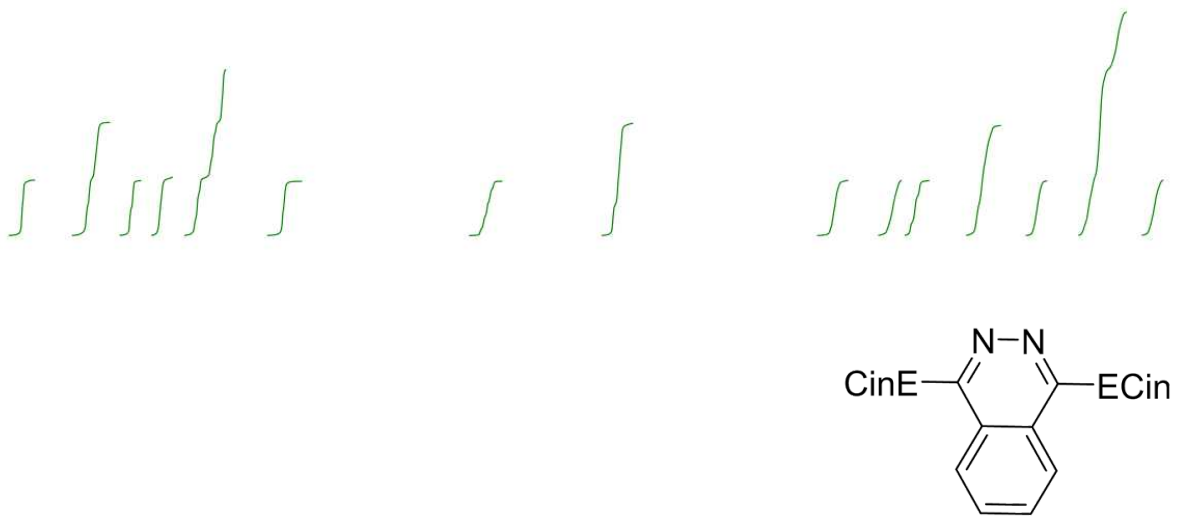

(ECin) ${ }_{2} \mathrm{PHAL}$

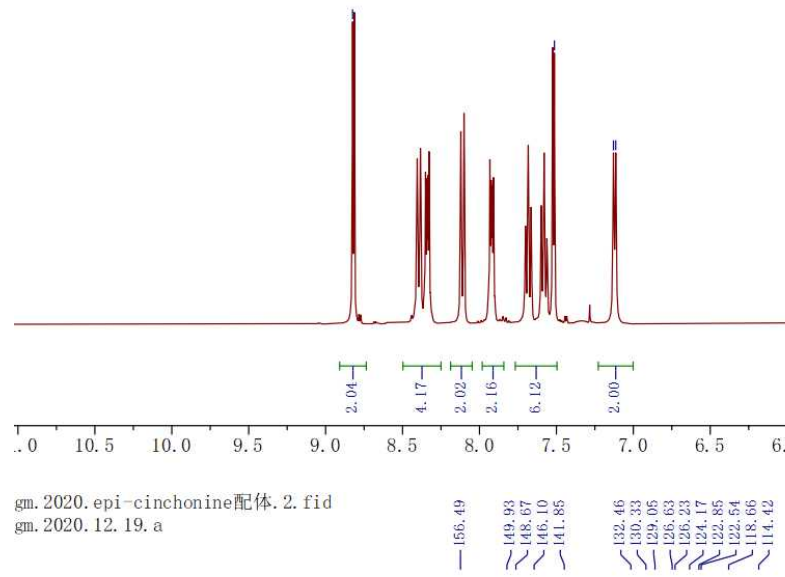

$400 \mathrm{MHz}, \mathrm{CDCl}_{3}$
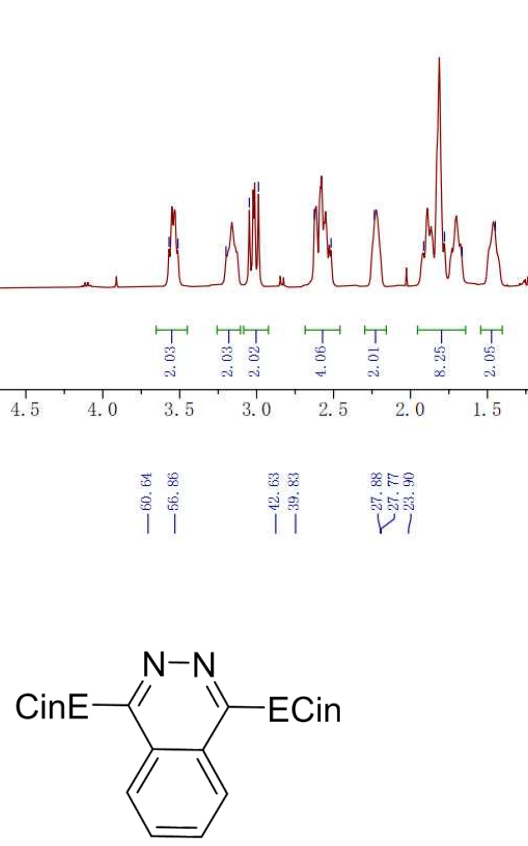

(ECin) ${ }_{2} \mathrm{PHAL}$

$100 \mathrm{MHz}, \mathrm{CDCl}_{3}$

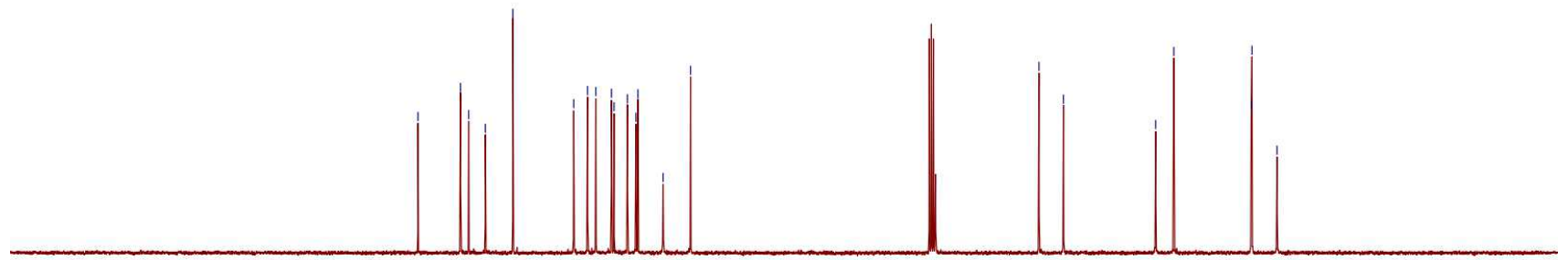

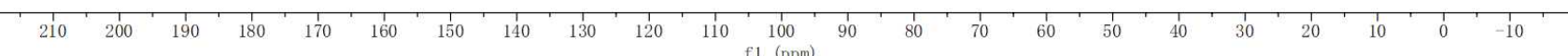


<smiles>C=C(CN(c1ccccc1)c1ccccc1C(=O)OC)c1ccccc1</smiles>

$400 \mathrm{MHz}, \mathrm{CDCl}_{3}$

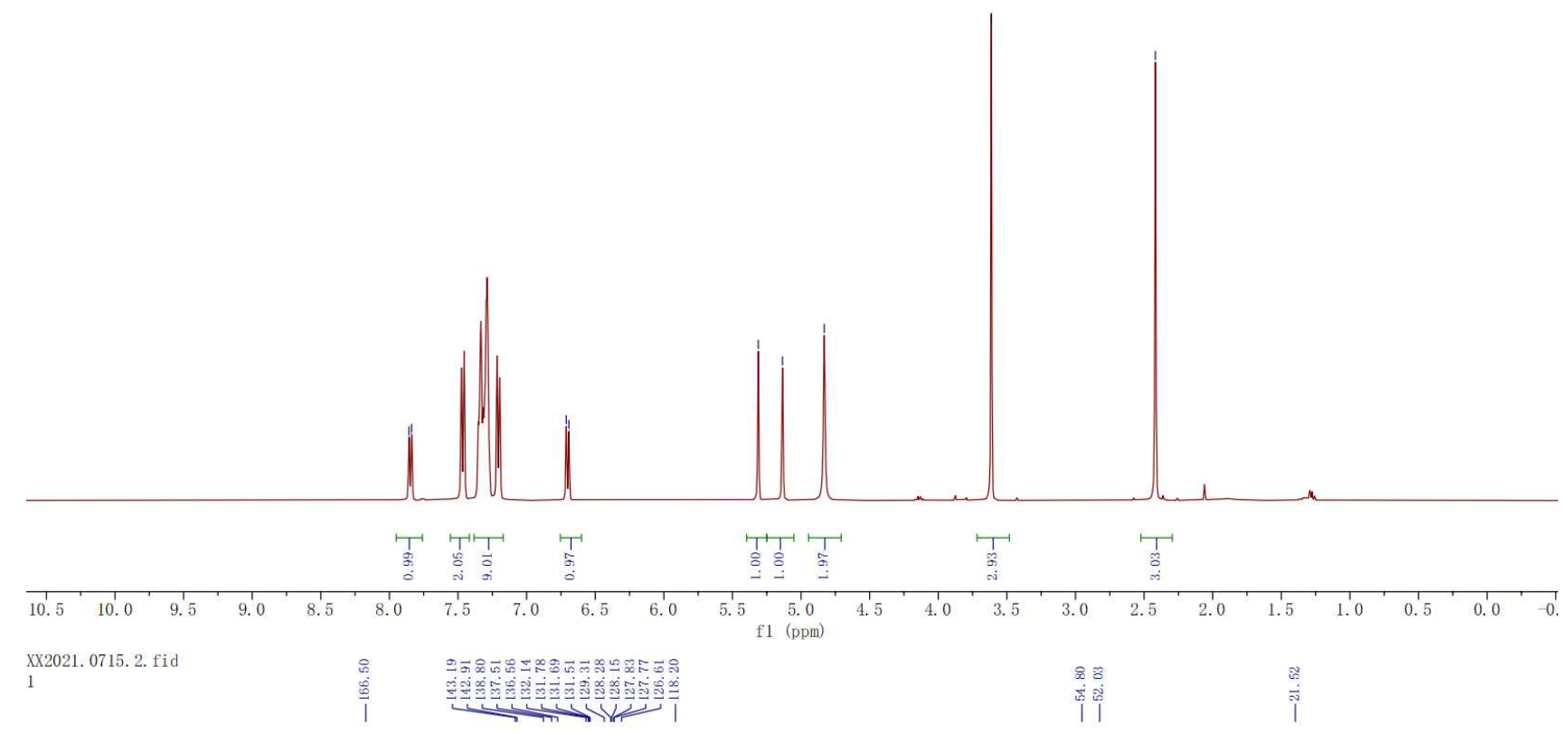<smiles>C=C(CN([As])c1ccccc1C(=O)OC)c1ccccc1</smiles>

III-a

$100 \mathrm{MHz}, \mathrm{CDCl}_{3}$

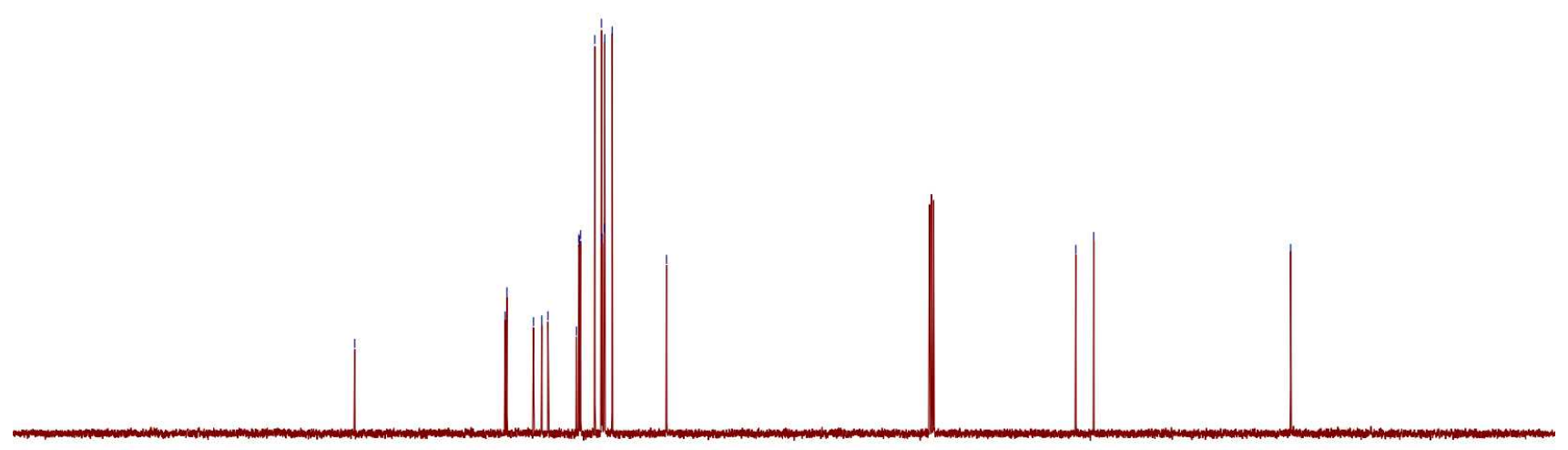




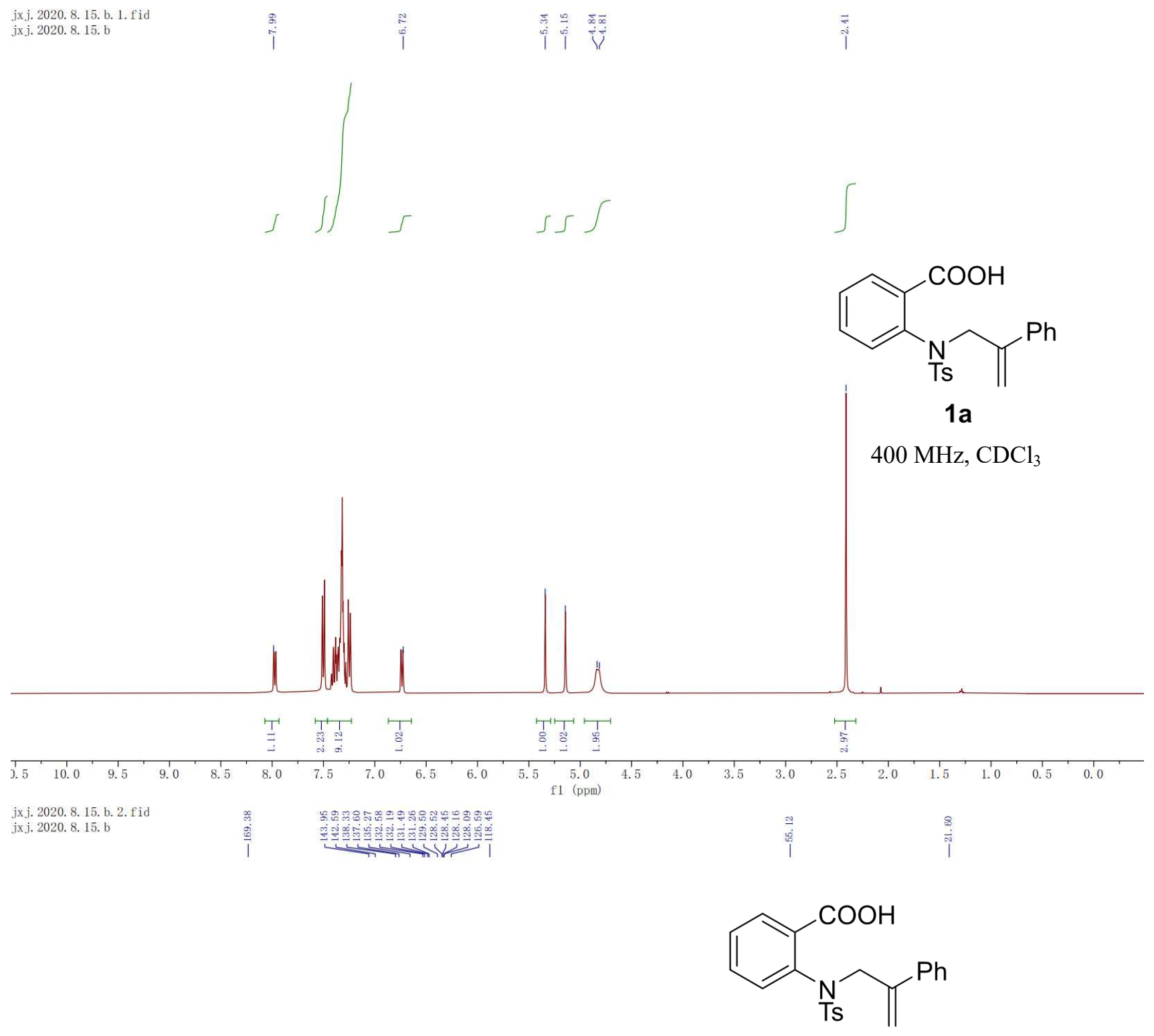

$1 a$

$100 \mathrm{MHz}, \mathrm{CDCl}_{3}$

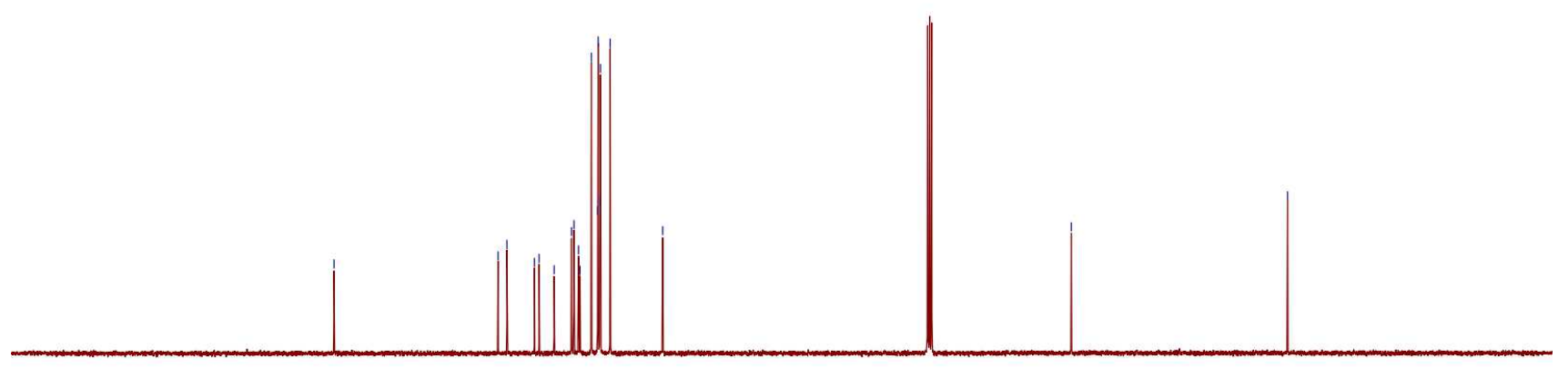

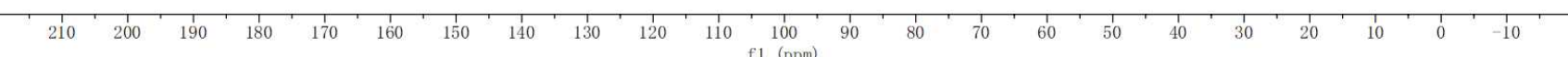



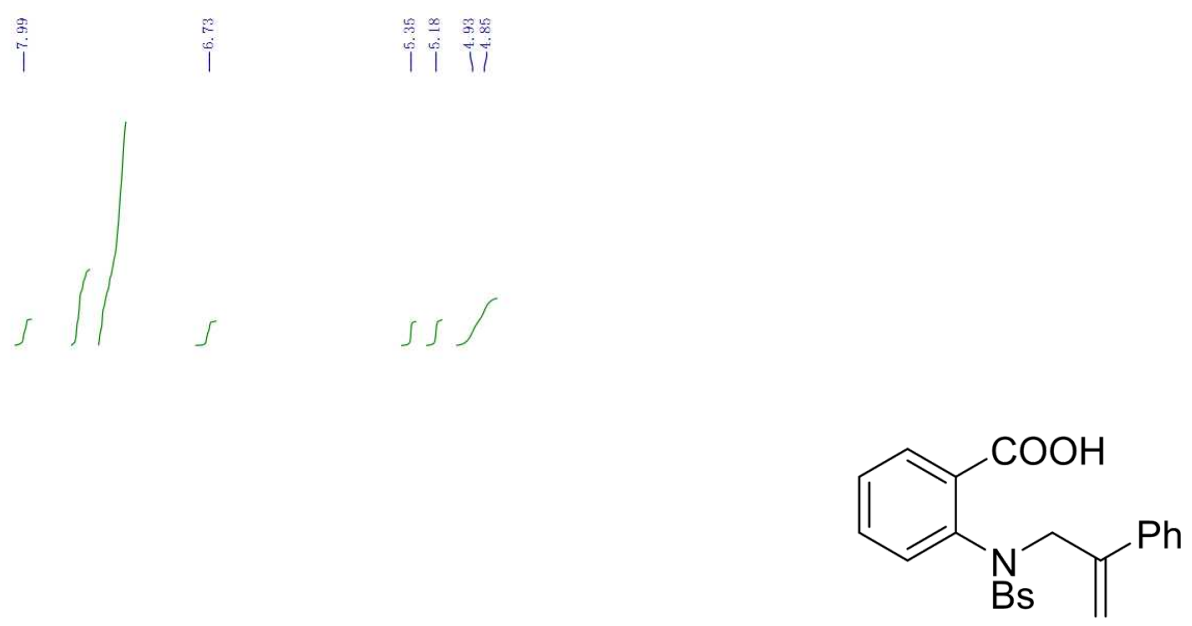

1b

$400 \mathrm{MHz}, \mathrm{CDCl}_{3}$

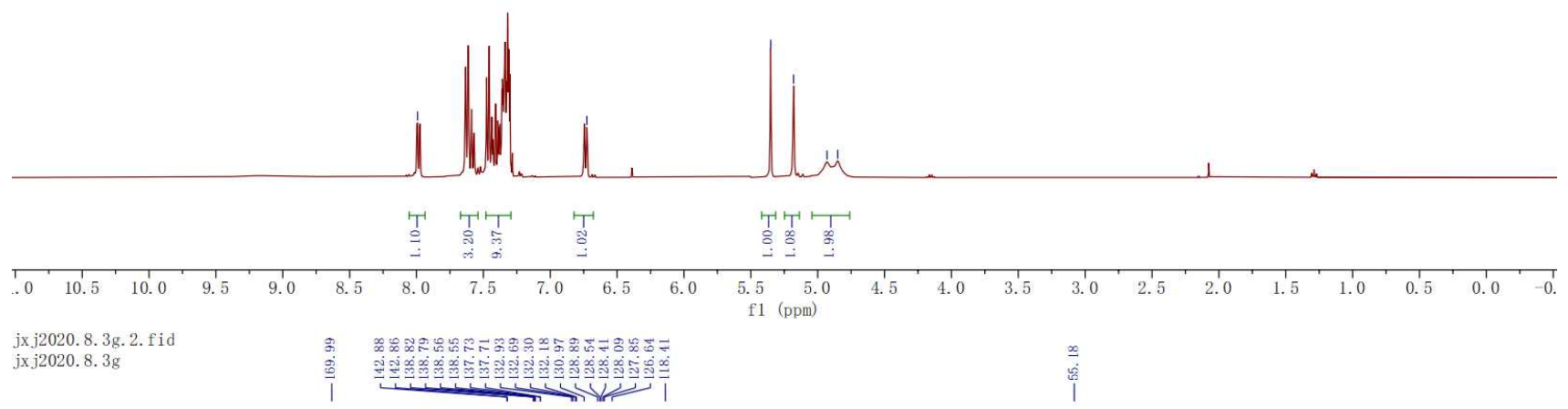<smiles>C=C(CN(c1ccccc1)c1ccccc1C(=O)O)c1ccccc1</smiles>

1b

$100 \mathrm{MHz}, \mathrm{CDCl}_{3}$

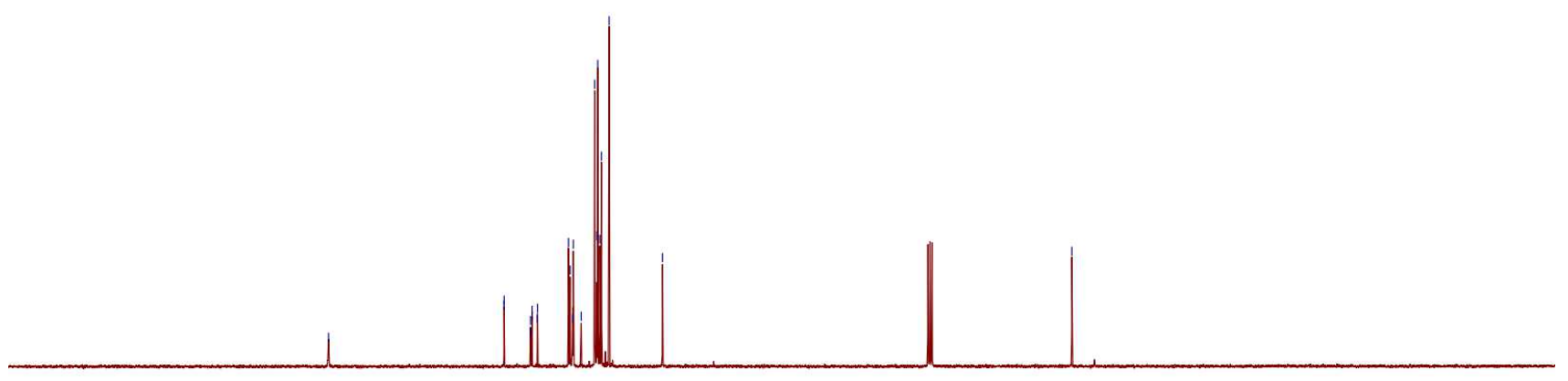

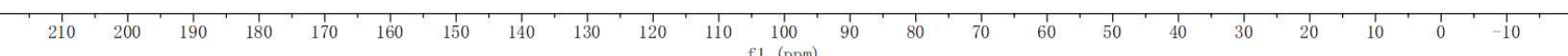



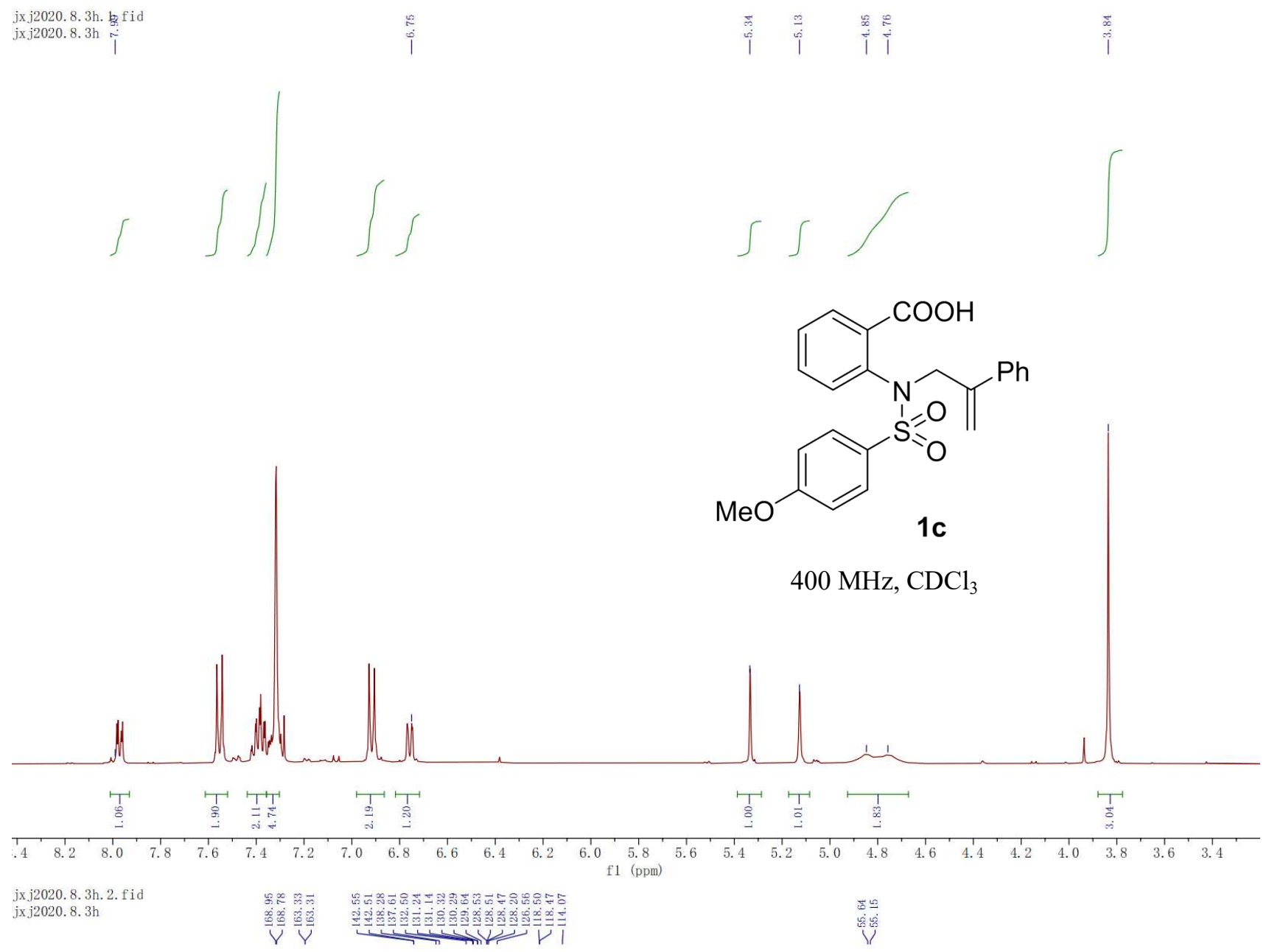<smiles>C=C(CN(c1ccccc1C(=O)O)S(=O)(=O)c1ccc(OC)cc1)c1ccccc1</smiles>

$100 \mathrm{MHz}, \mathrm{CDCl}_{3}$

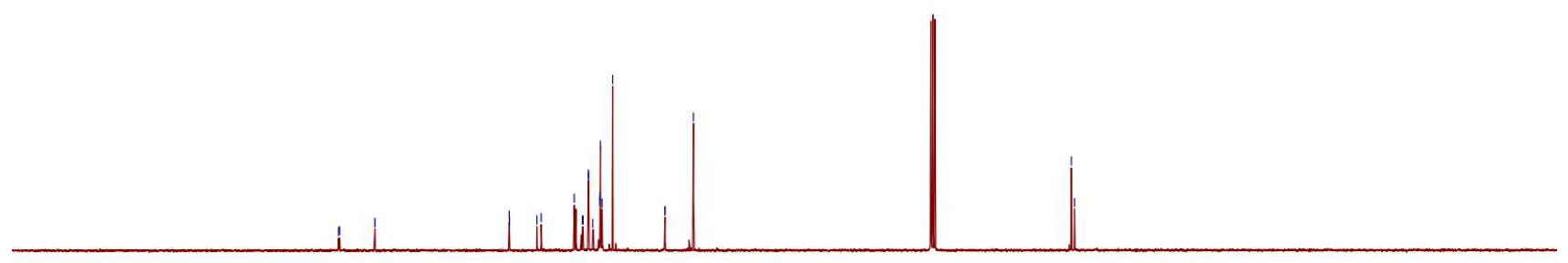

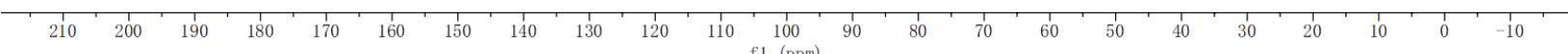



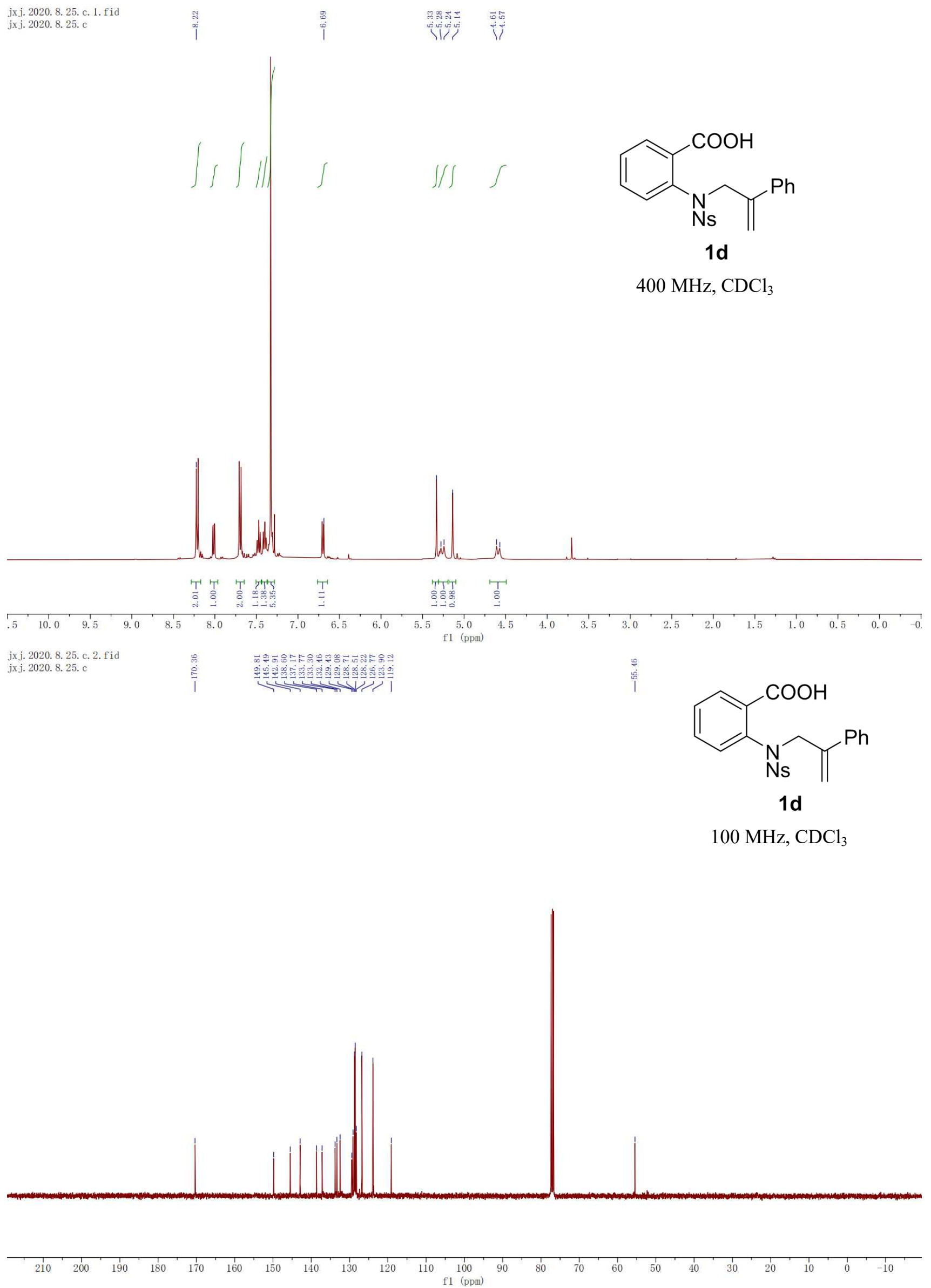


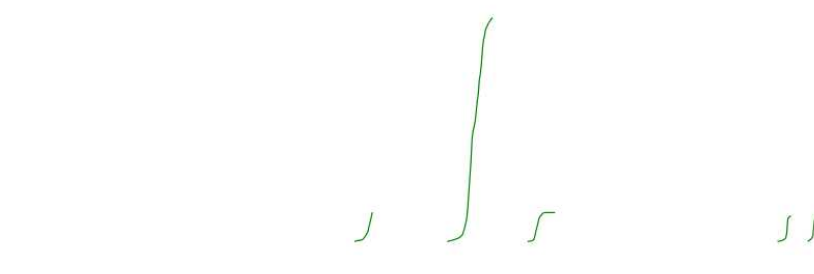<smiles>C=C(CN(C)c1ccccc1C(=O)O)c1ccccc1</smiles>

$1 e$

$400 \mathrm{MHz}, \mathrm{CDCl}_{3}$
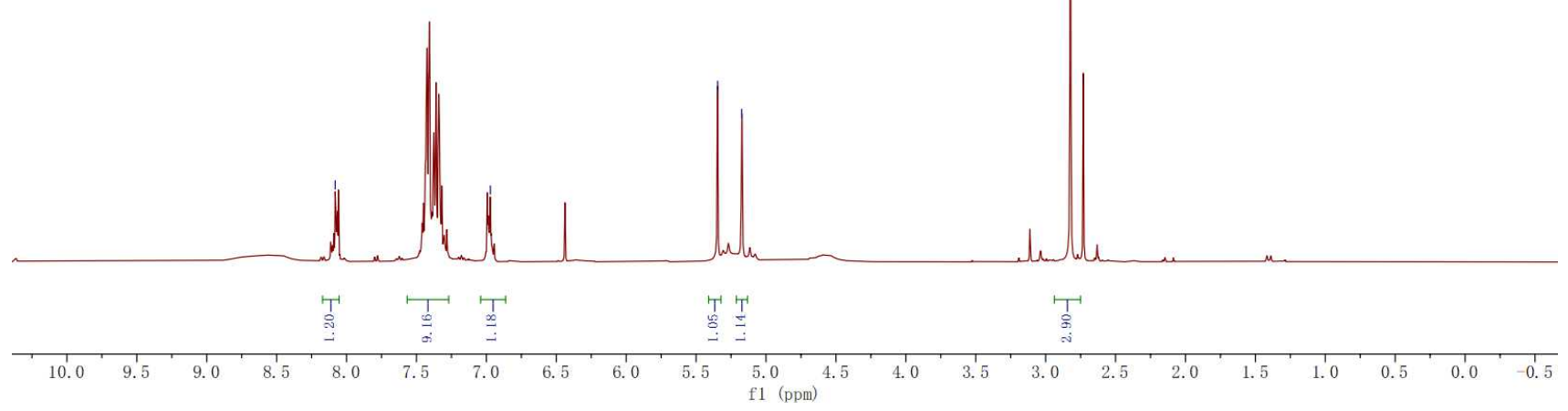

jx j2020. 8. 3k. 2. fid jx j2020. 8. 3k

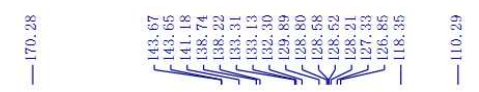

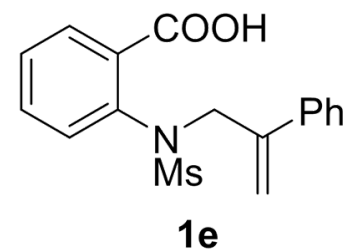

$100 \mathrm{MHz}, \mathrm{CDCl}_{3}$

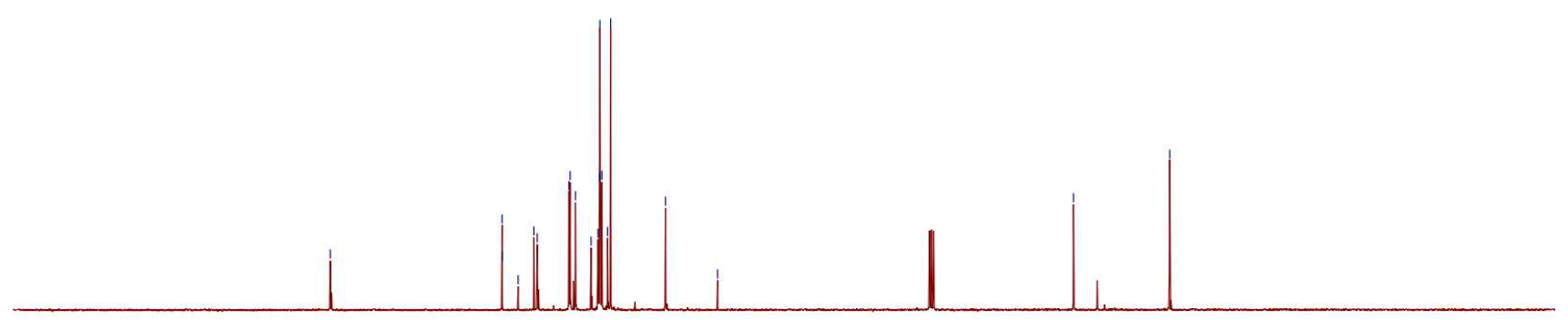

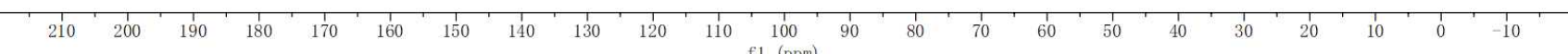



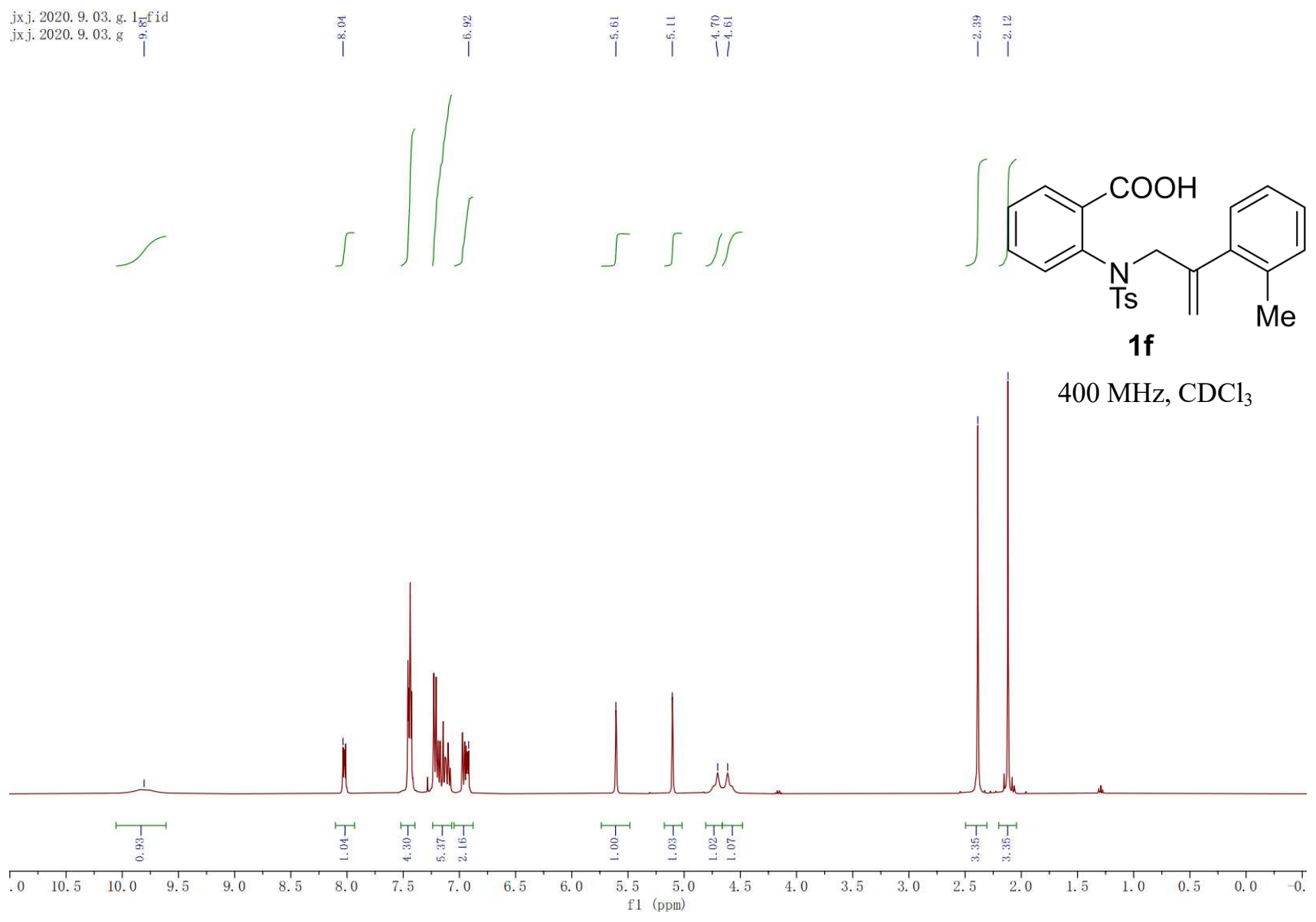

jx j. 2020. 9. 03. g. 2. fid

jx j. 2020.9. 03. g

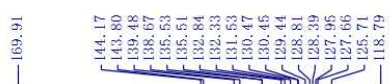

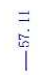

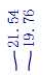

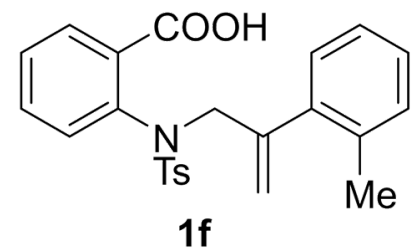

$100 \mathrm{MHz}, \mathrm{CDCl}_{3}$

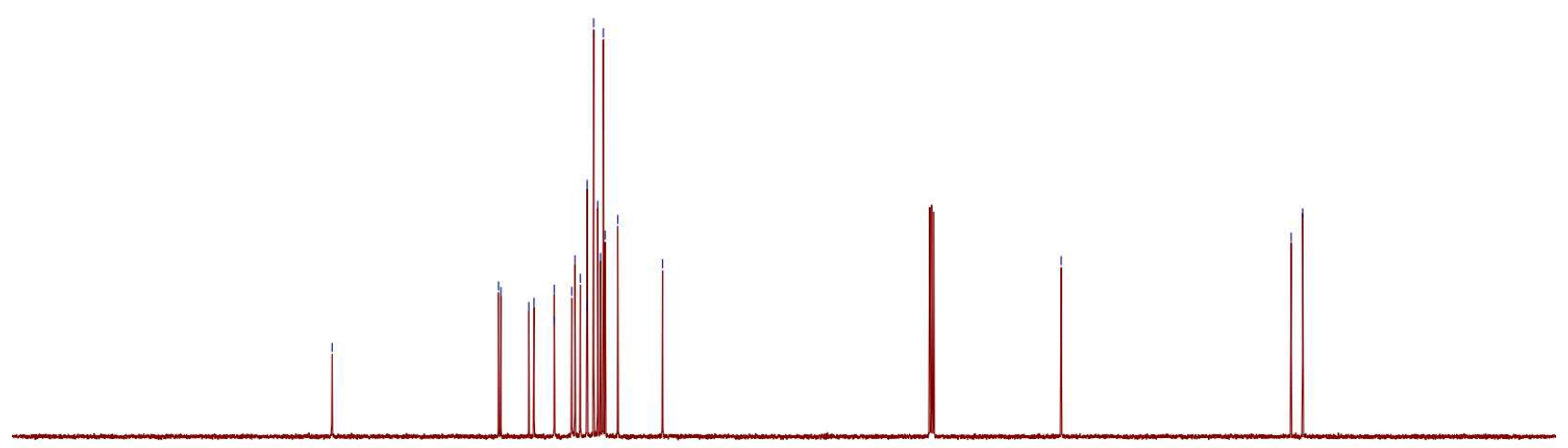

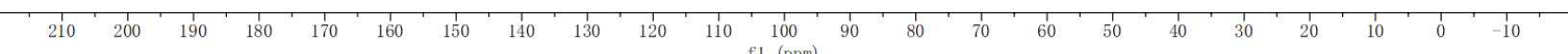



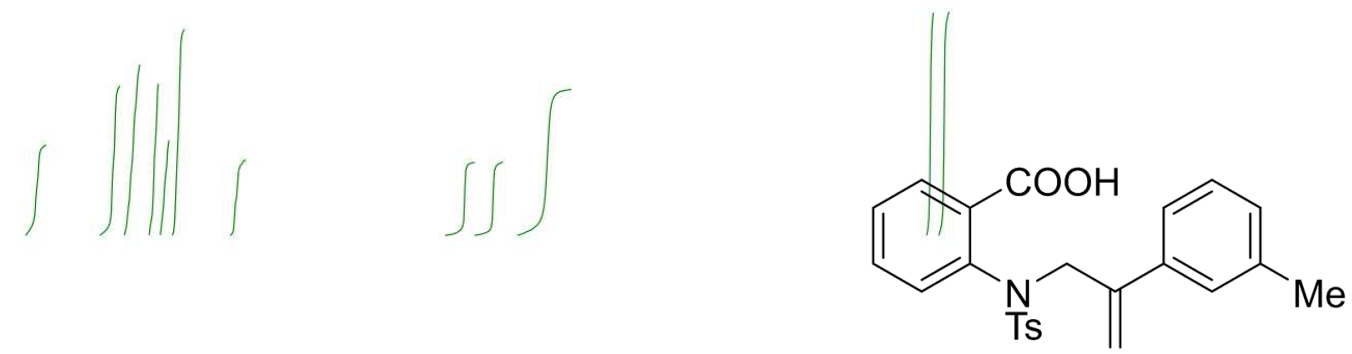

$1 \mathrm{~g}$

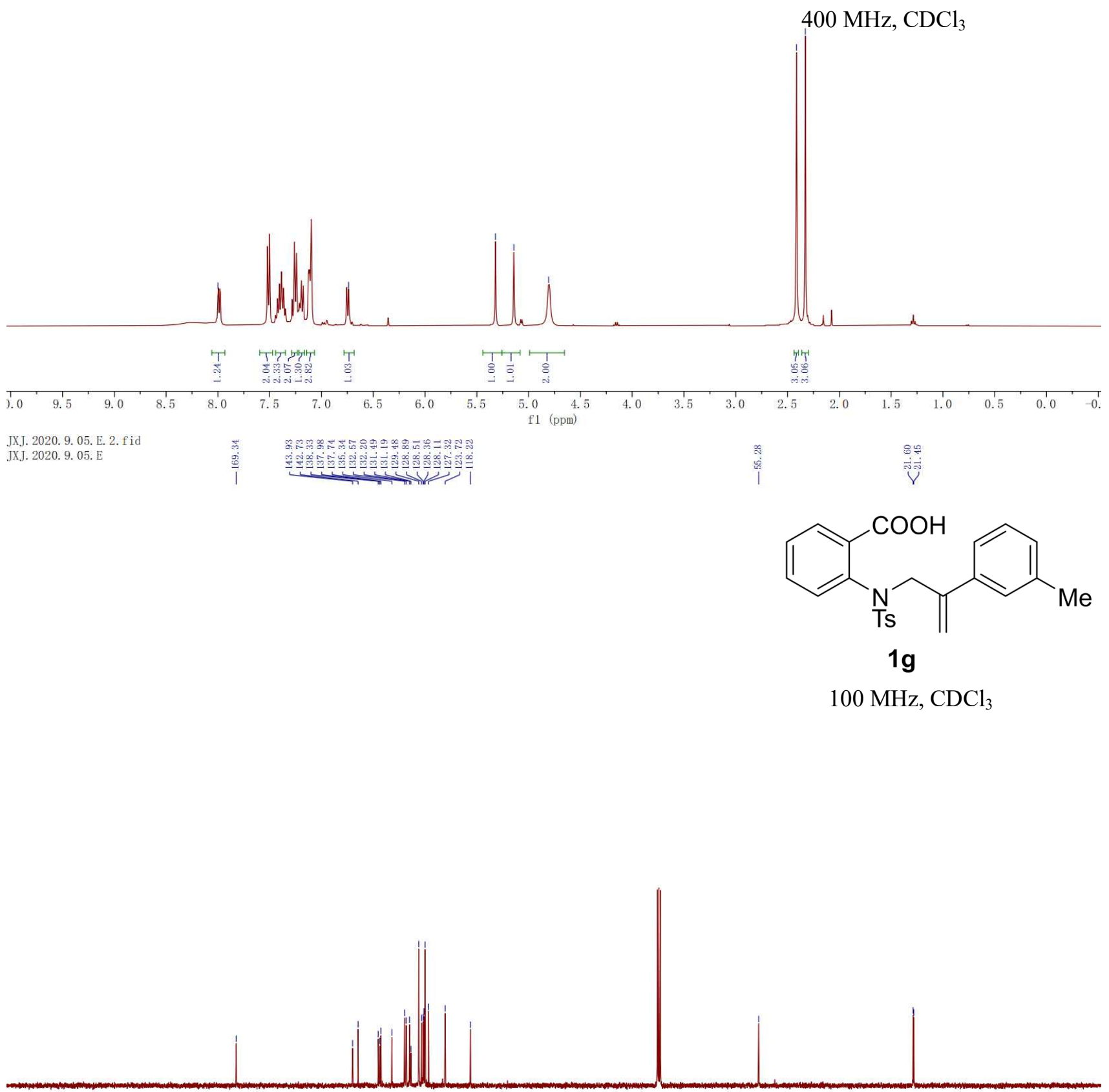

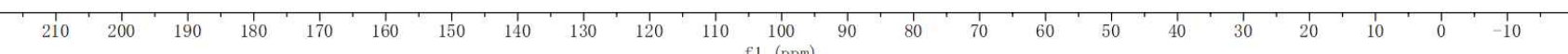


<smiles>C=C(CN(C)c1ccccc1C(=O)O)c1ccc(C)cc1</smiles>

1h

$400 \mathrm{MHz}, \mathrm{CDCl}_{3}$

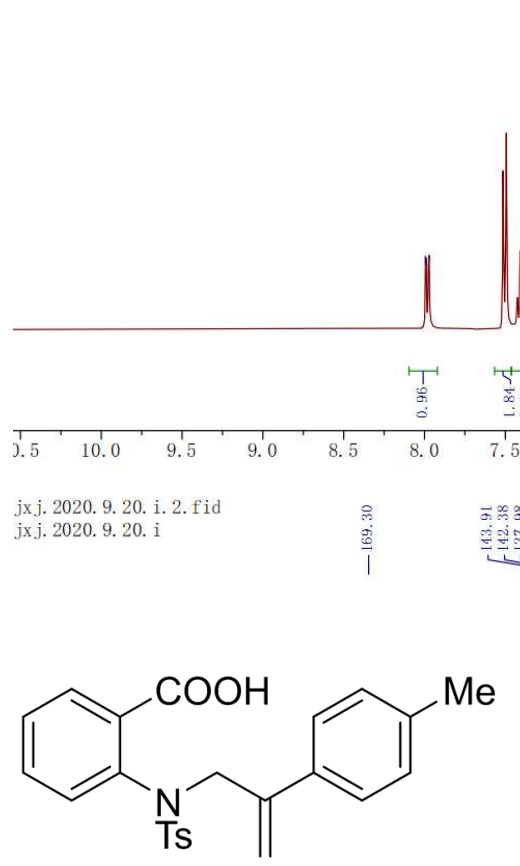

1h

$100 \mathrm{MHz}, \mathrm{CDCl}_{3}$

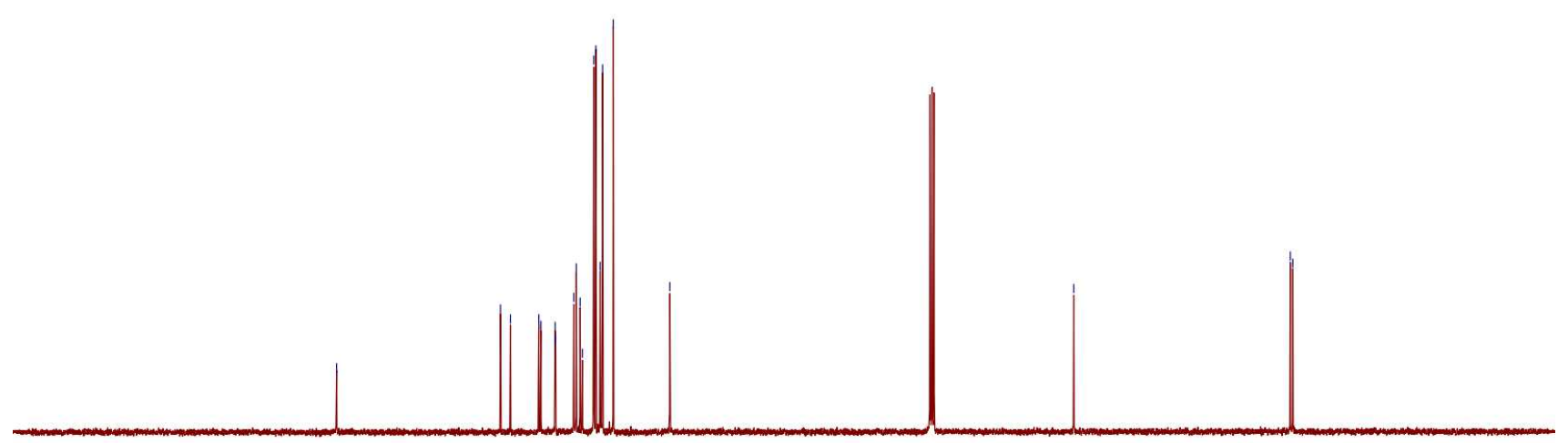

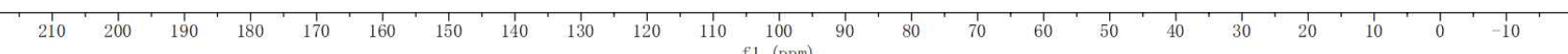




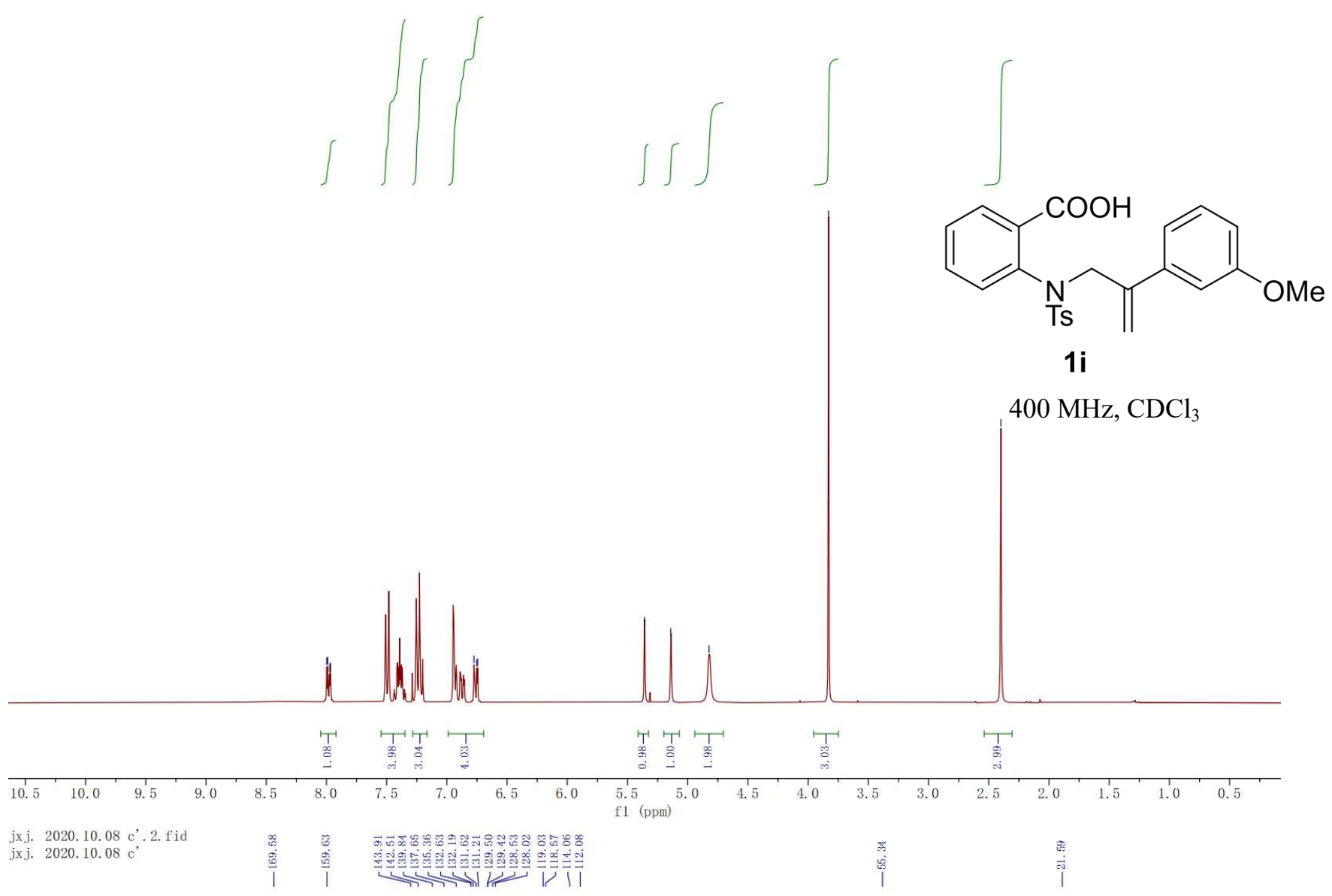<smiles>C=C(CN([As])c1ccccc1C(=O)O)c1cccc(OC)c1</smiles>

$1 i$

$100 \mathrm{MHz}, \mathrm{CDCl}_{3}$

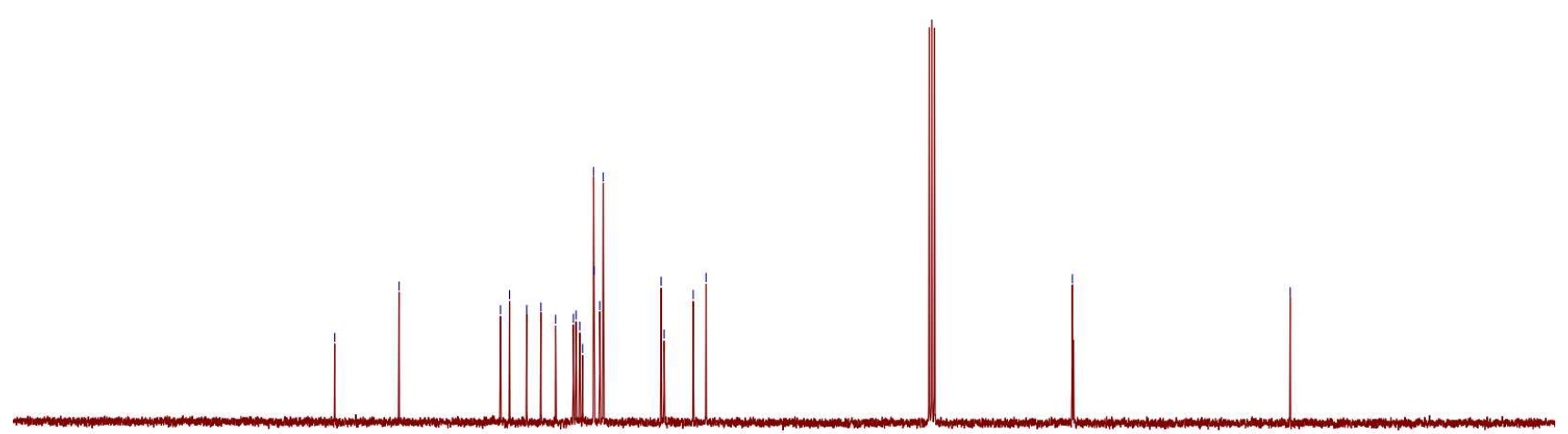

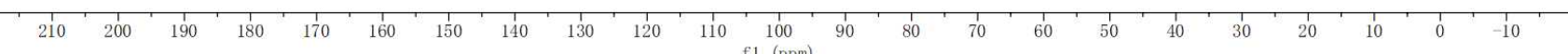




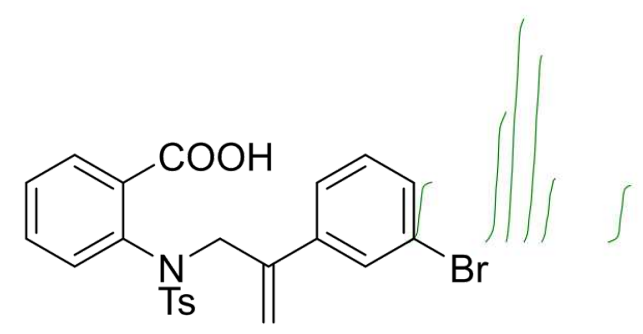

$1 \mathrm{j}$

$400 \mathrm{MHz}, \mathrm{CDCl}_{3}$

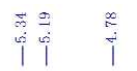

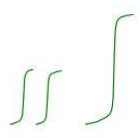

$$
\int
$$
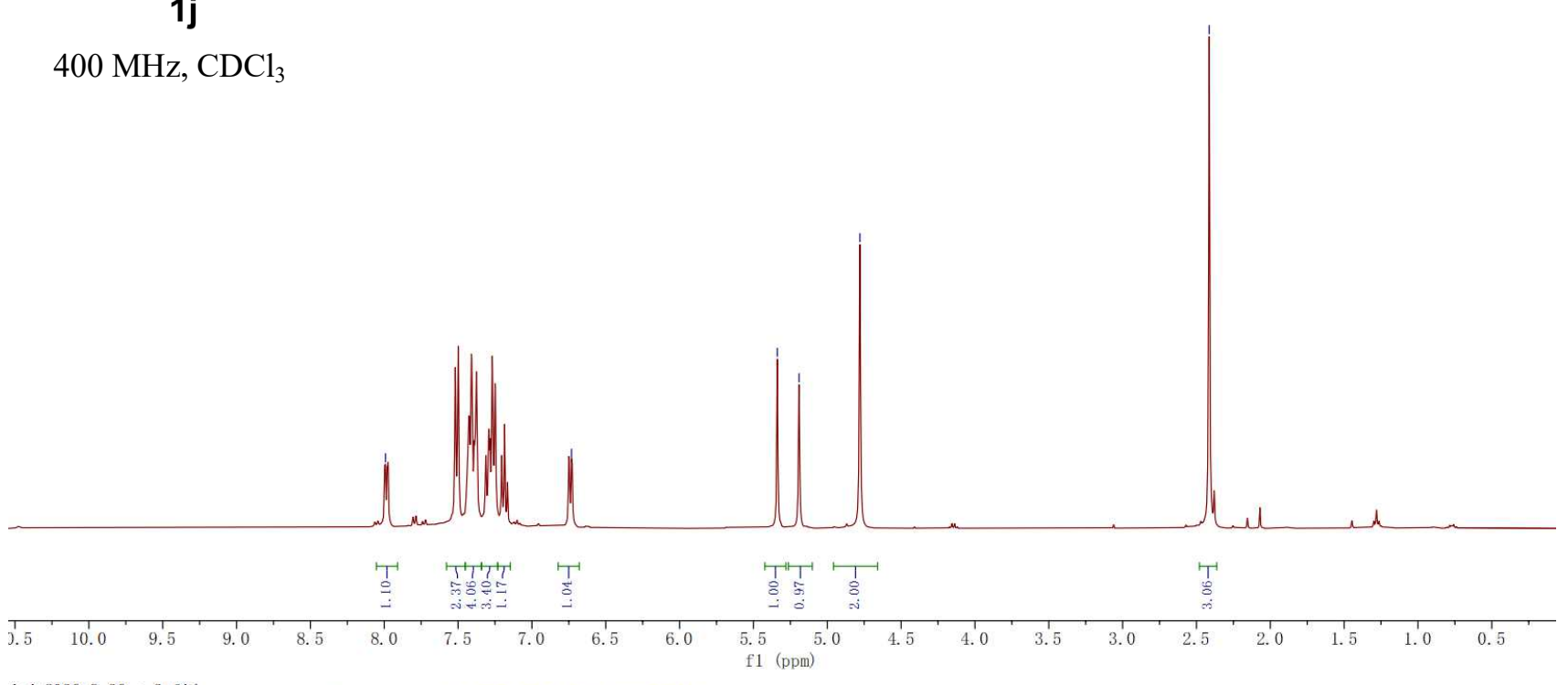

jx. j. 2020. 9. 20. c. 2. fid

jxj. 2020.9.20.c. 2.fid
jxj.2020.9.20. c
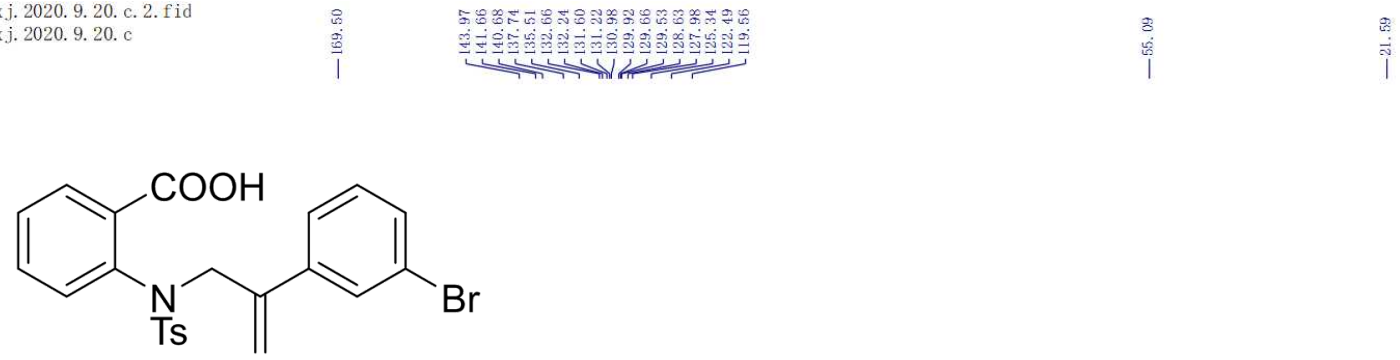

1j

$100 \mathrm{MHz}, \mathrm{CDCl}_{3}$

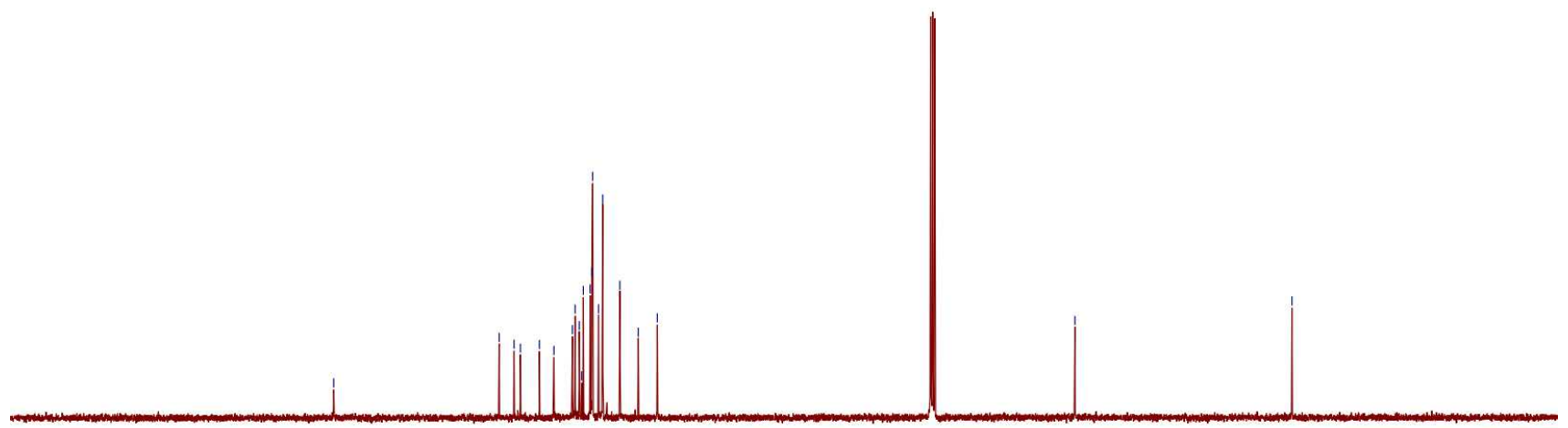

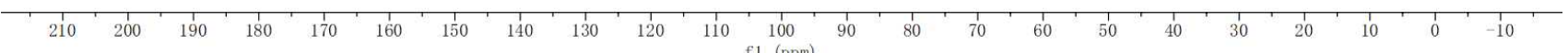




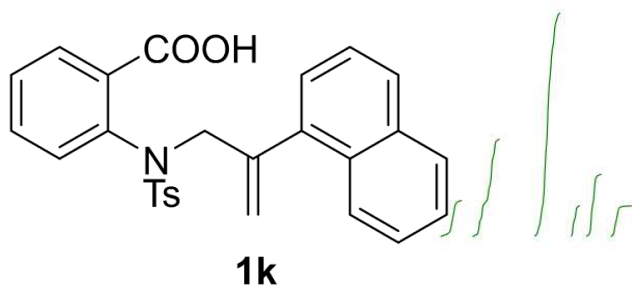

$400 \mathrm{MHz}, \mathrm{CDCl}_{3}$

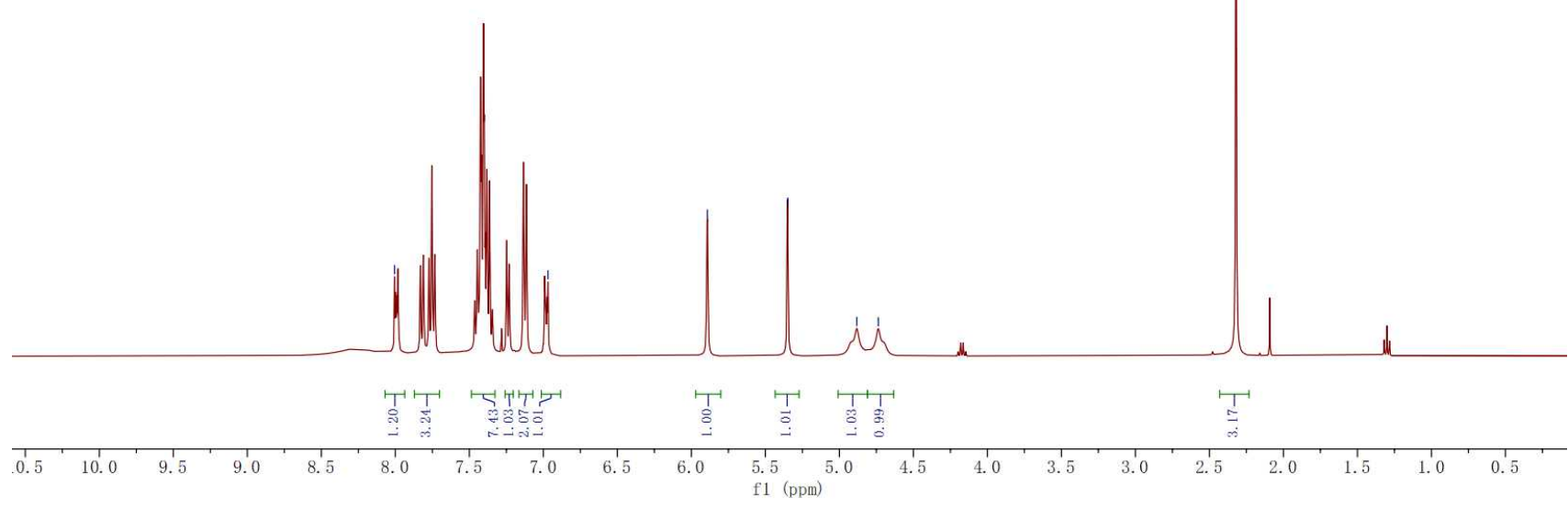

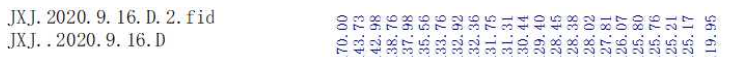
JXJ. 2020.9.16.D<smiles>C=C(CN(C)c1ccccc1C(=O)O)c1cccc2ccccc12</smiles>

$100 \mathrm{MHz}, \mathrm{CDCl}_{3}$

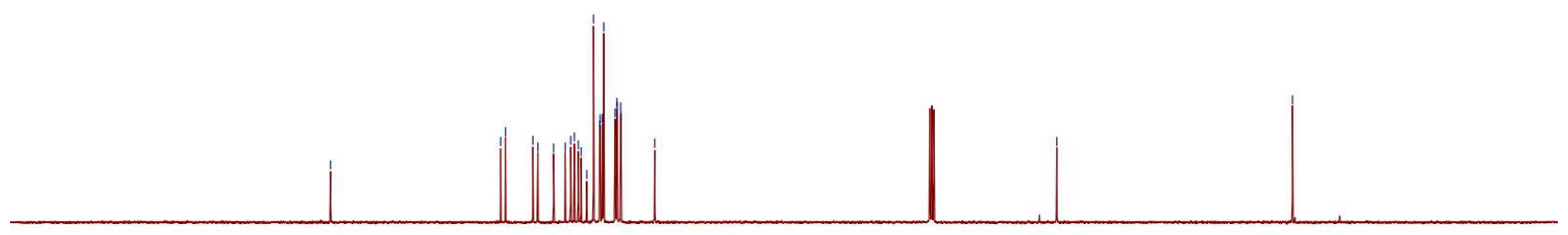



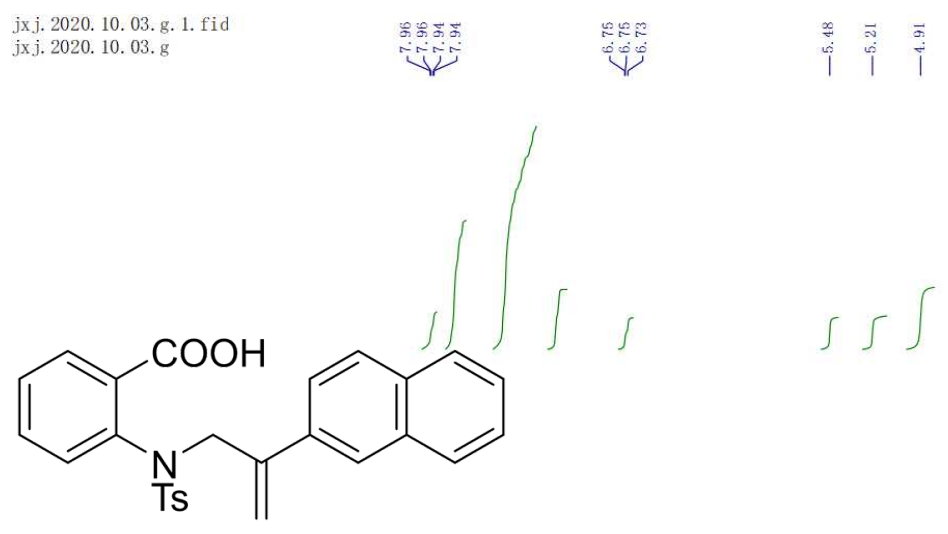

11

$400 \mathrm{MHz}, \mathrm{CDCl}_{3}$

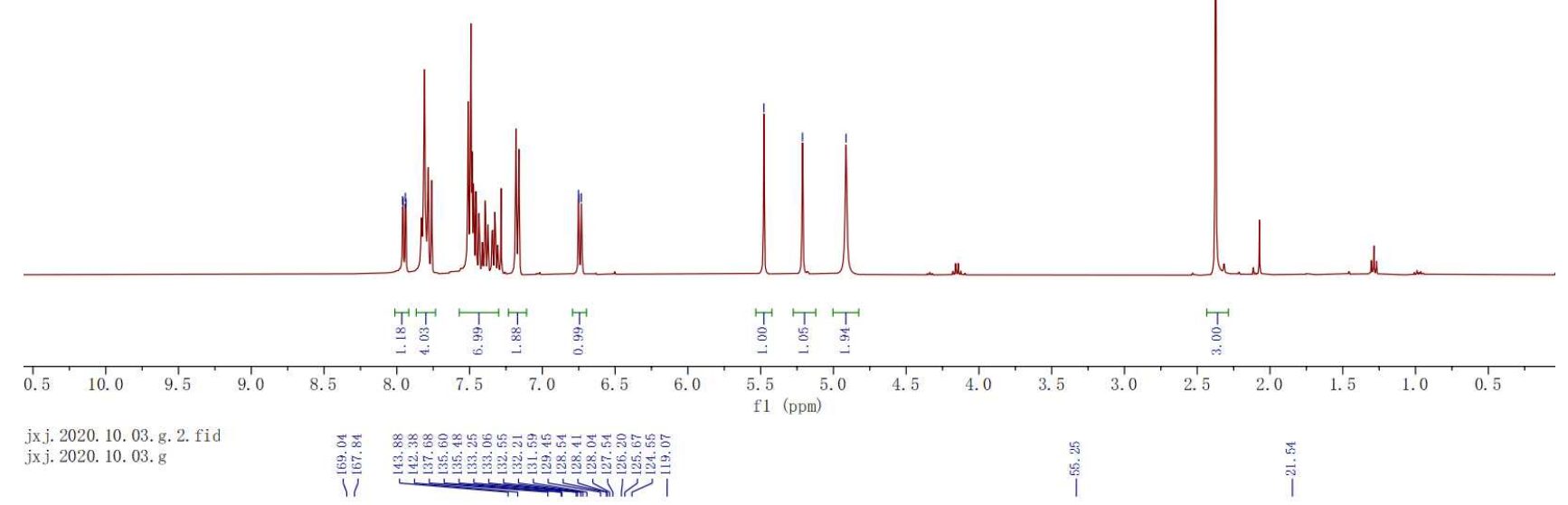<smiles>C=C(CN([As])c1ccccc1C(=O)O)c1ccc2ccccc2c1</smiles>

11

$100 \mathrm{MHz}, \mathrm{CDCl}_{3}$

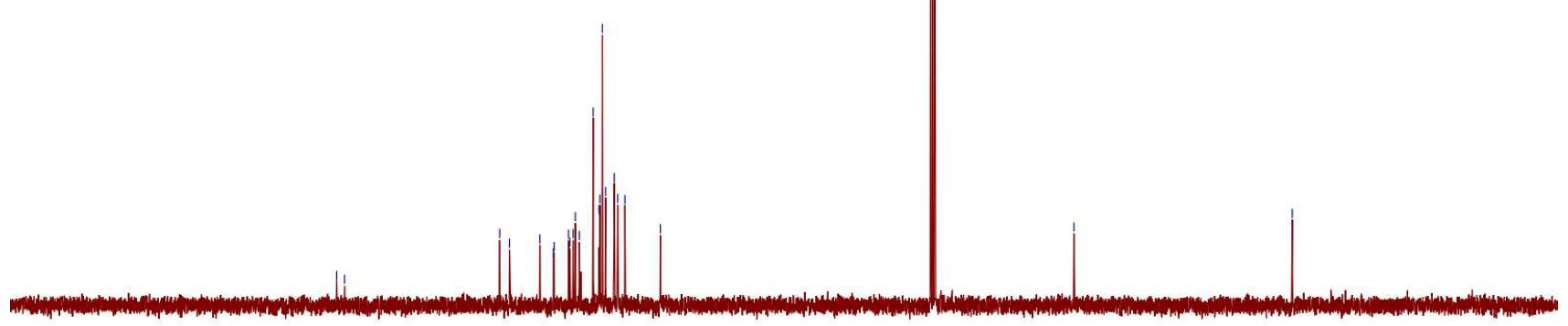

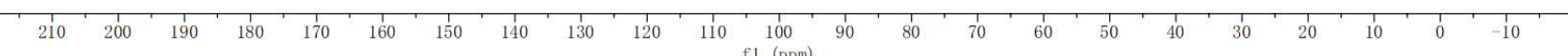




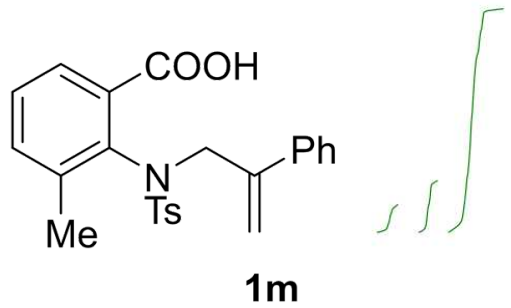

$400 \mathrm{MHz}, \mathrm{CDCl}_{3}$

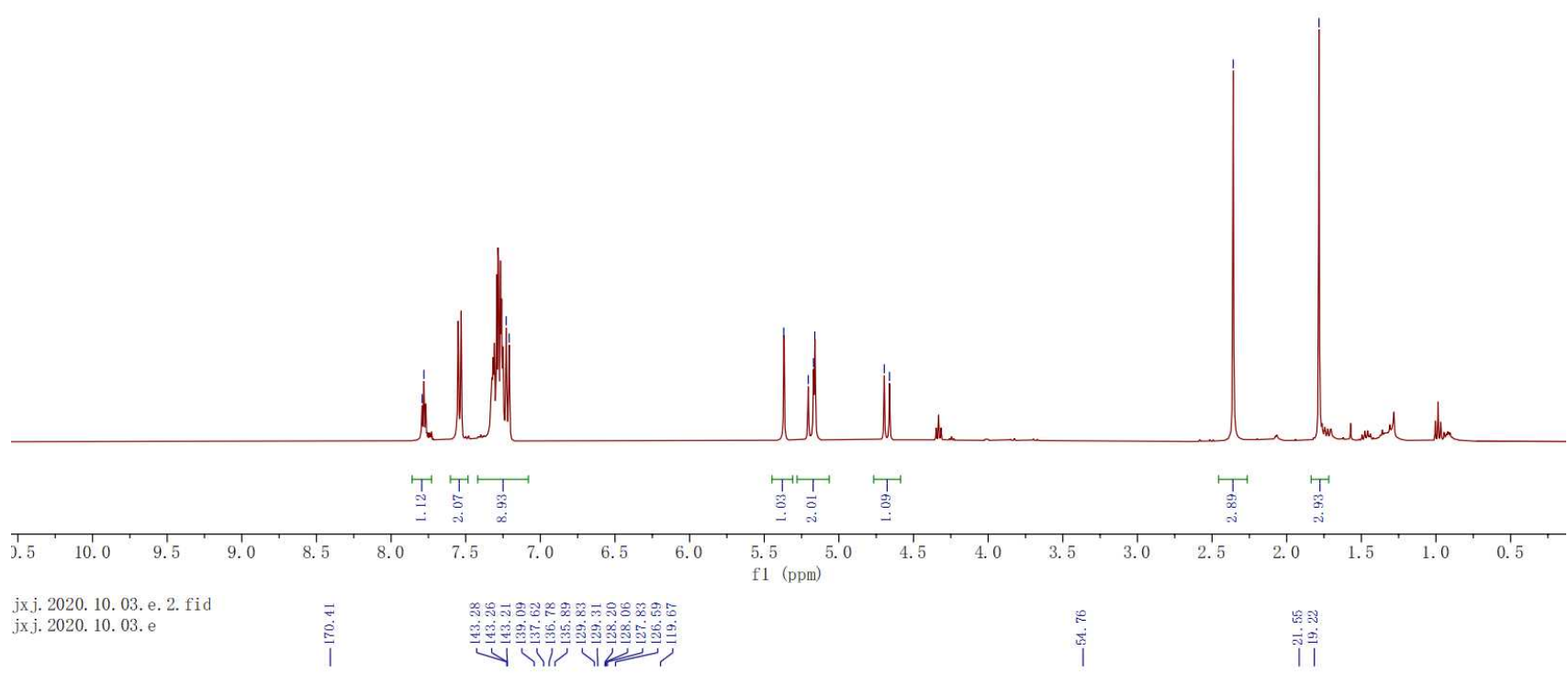<smiles>C=C(CN([As])c1c(C)cccc1C(=O)O)c1ccccc1</smiles>

$100 \mathrm{MHz}, \mathrm{CDCl}_{3}$

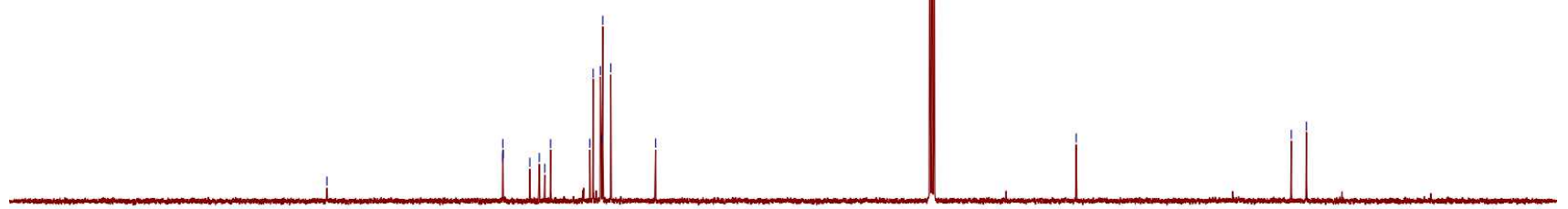

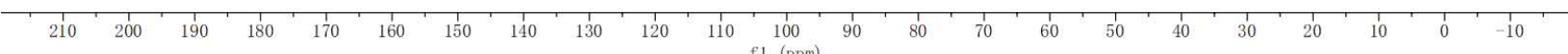




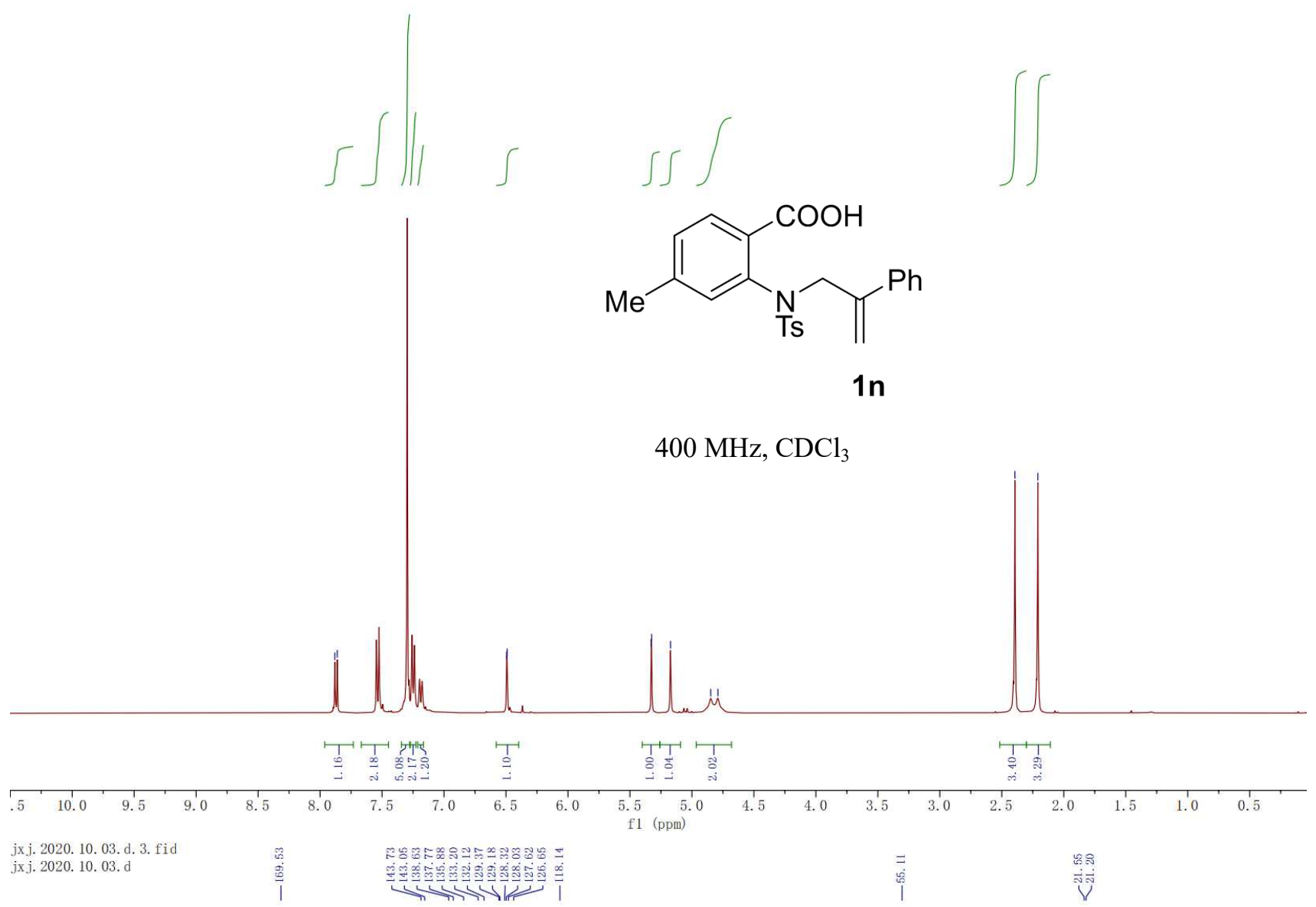<smiles>C=C(CN([As])c1cc(C)ccc1C(=O)O)c1ccccc1</smiles>

1n

$100 \mathrm{MHz}, \mathrm{CDCl}_{3}$

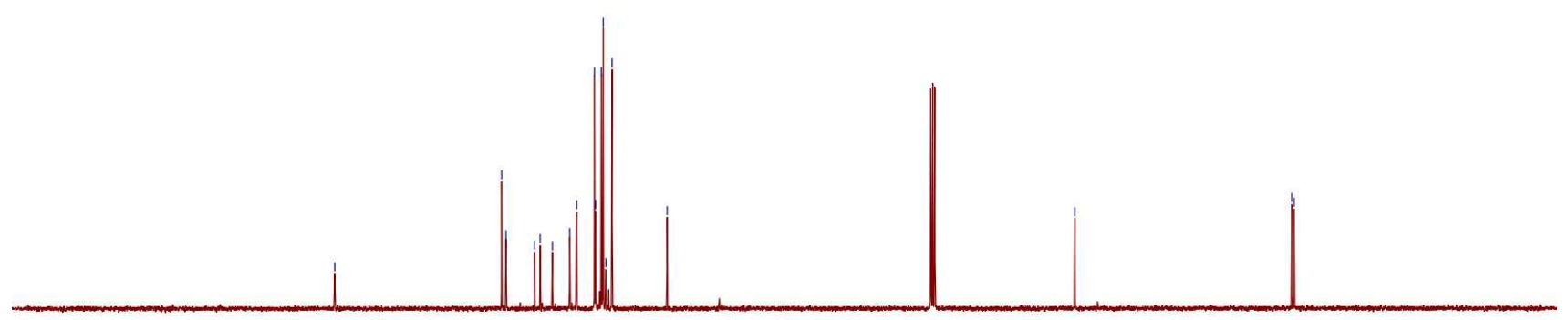

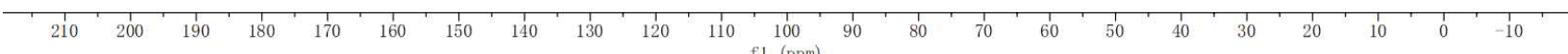




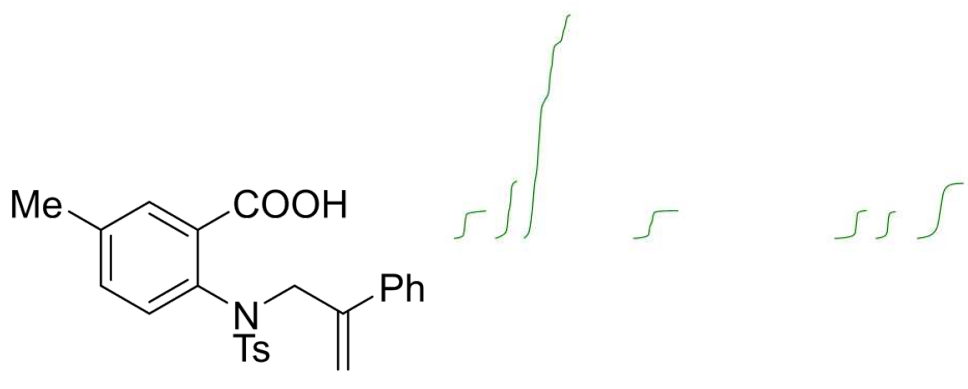

10

$400 \mathrm{MHz}, \mathrm{CDCl}_{3}$

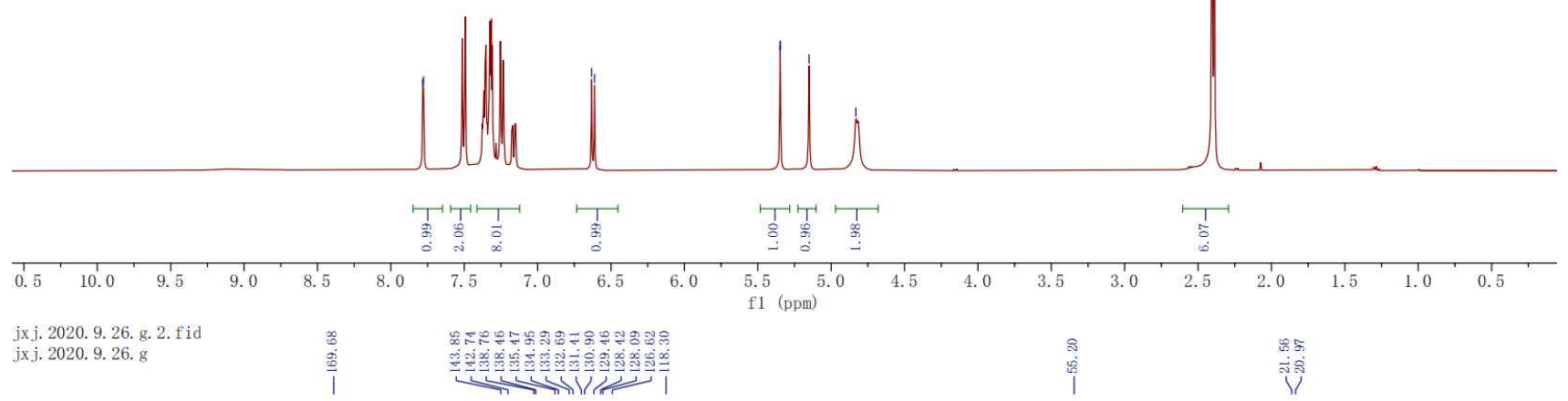

$\overbrace{\text { TS }}^{\mathrm{COOH}}$

10

$100 \mathrm{MHz}, \mathrm{CDCl}_{3}$

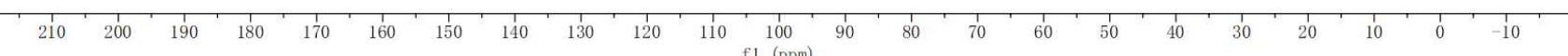




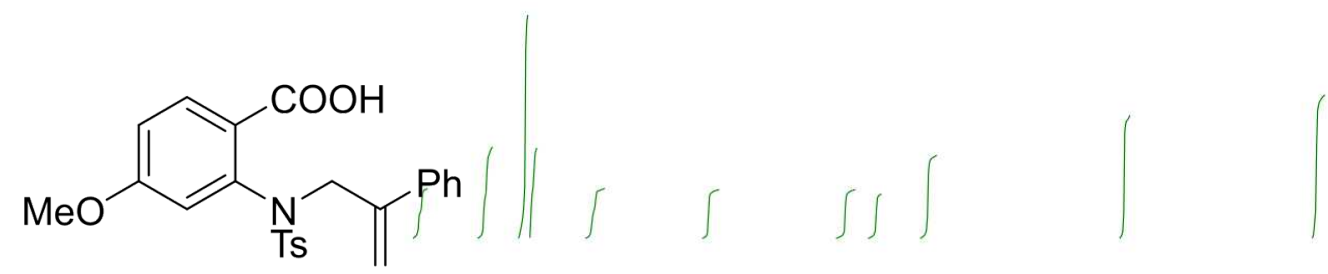

$1 p$

$400 \mathrm{MHz}, \mathrm{CDCl}_{3}$
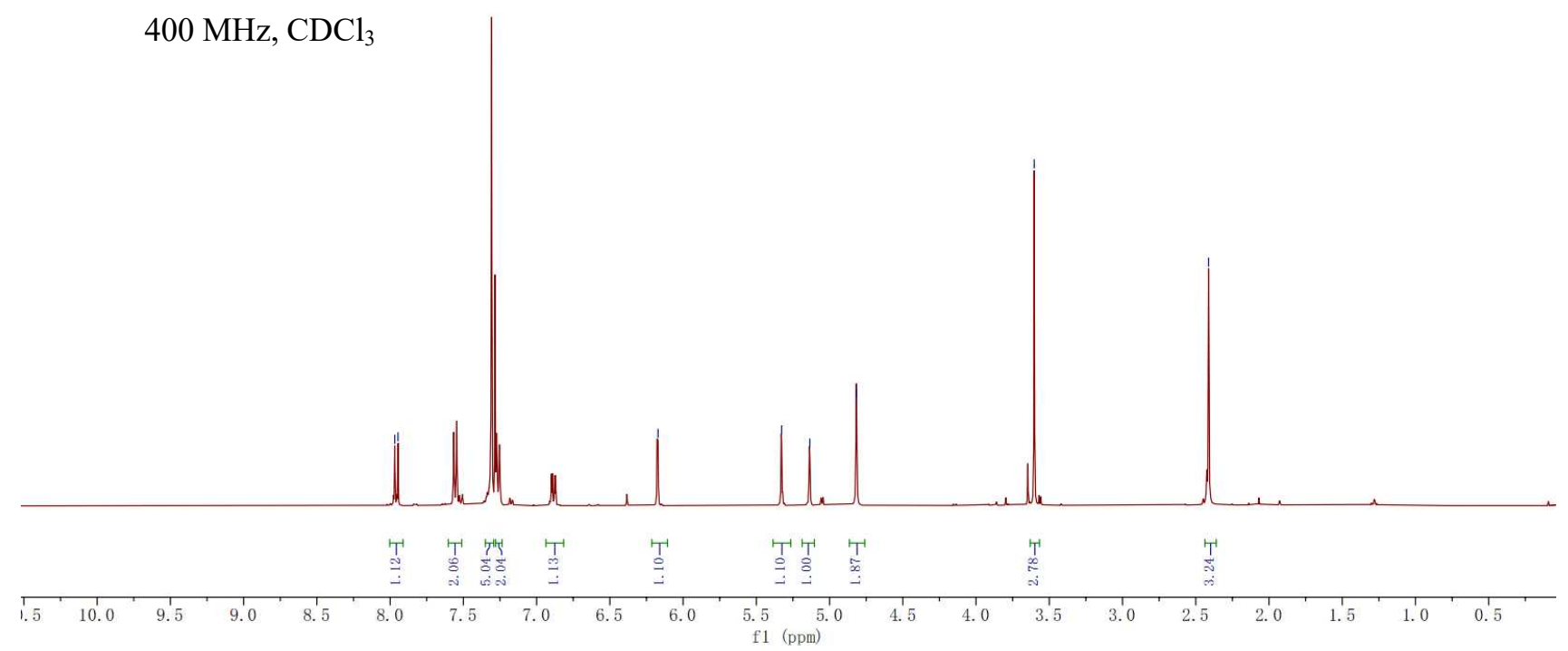

jx j. 2020. 11. 10.d. 2. fid

jx j. 2020.11. 10. d

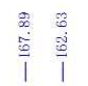

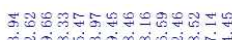

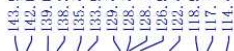<smiles>C=C(CN([As])c1cc(OC)ccc1C(=O)O)c1ccccc1</smiles>

$1 p$

$100 \mathrm{MHz}, \mathrm{CDCl}_{3}$

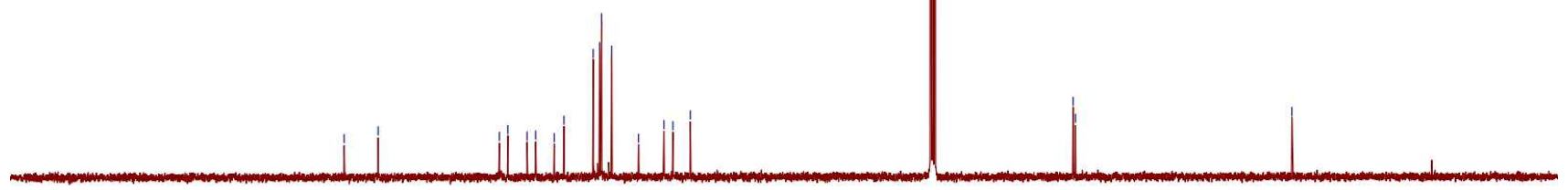

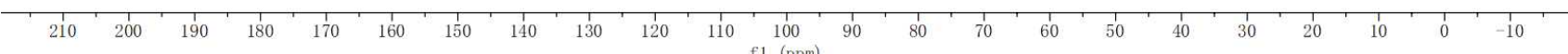


<smiles>C=C(CN([As])c1cc(F)ccc1C(=O)O)c1ccccc1</smiles>

$400 \mathrm{MHz}, \mathrm{CDCl}_{3}$

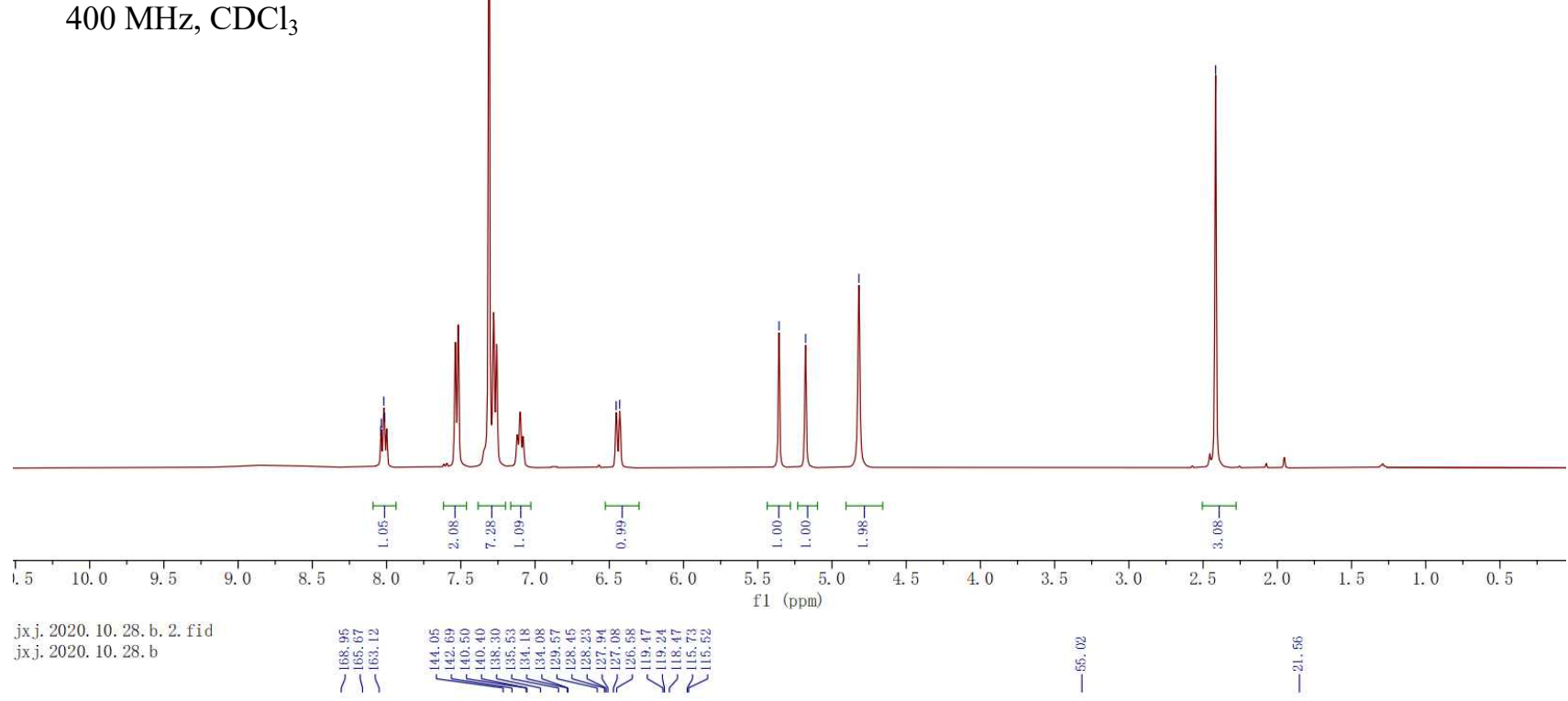<smiles>C=C(CN([As])c1cc(F)ccc1C(=O)O)c1ccccc1</smiles>

$1 q$

$100 \mathrm{MHz}, \mathrm{CDCl}_{3}$

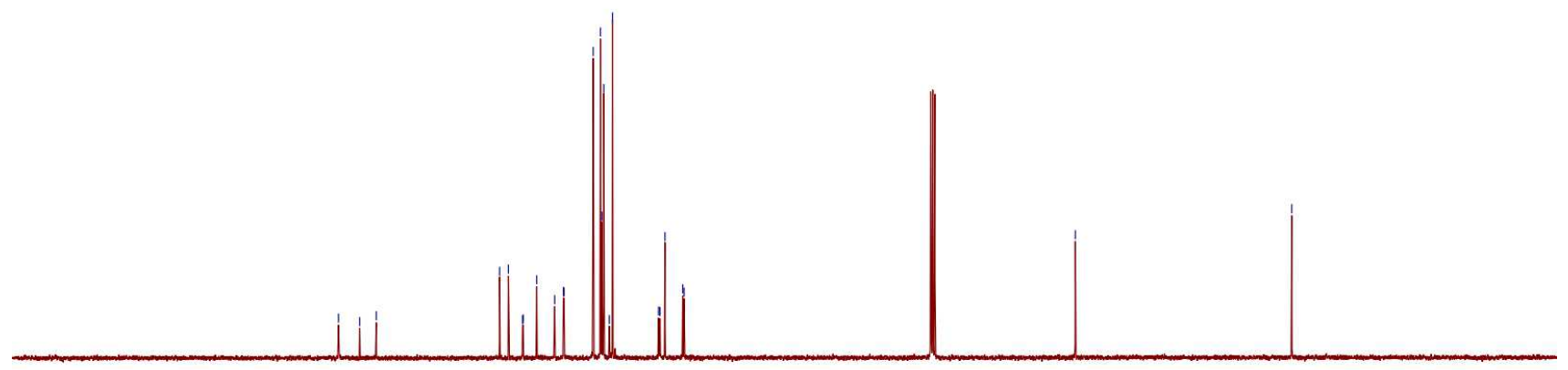

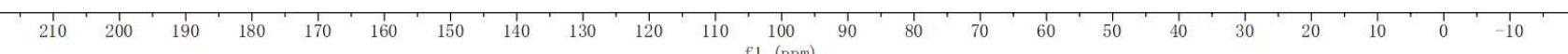


<smiles>C=C(CN([As])c1cc(Cl)ccc1C(=O)O)c1ccccc1</smiles>

$$
1 \mathrm{r}
$$

$400 \mathrm{MHz}, \mathrm{CDCl}_{3}$
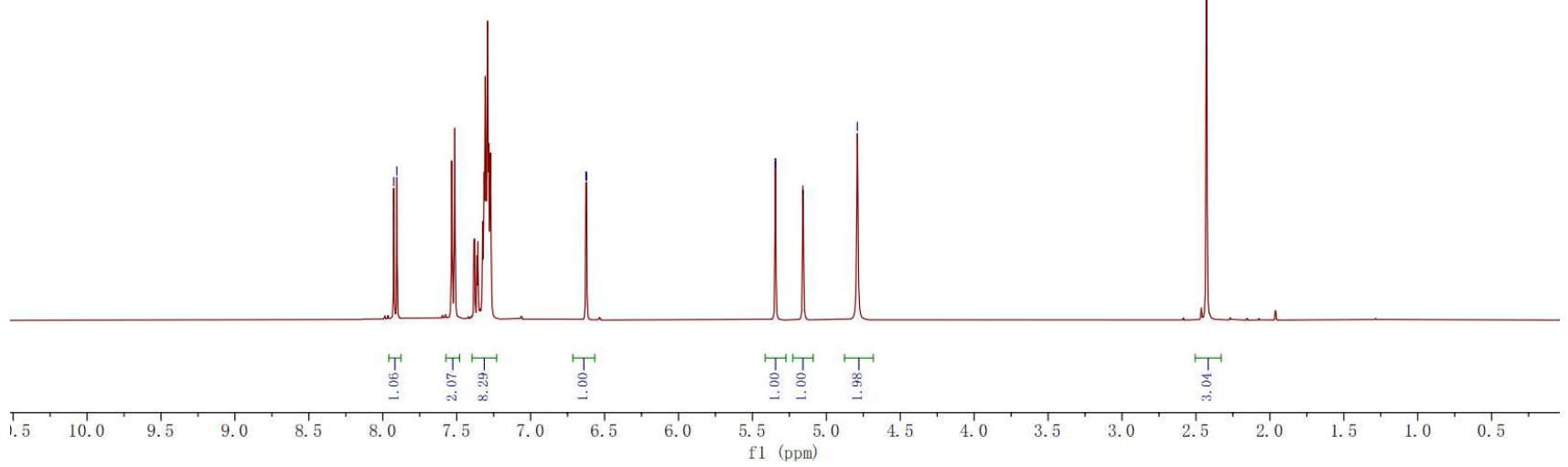

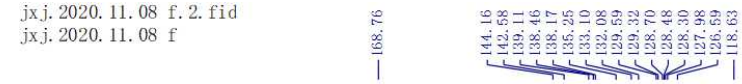<smiles>C=C(CN([As])c1cc(Cl)ccc1C(=O)O)c1ccccc1</smiles>

$100 \mathrm{MHz}, \mathrm{CDCl}_{3}$
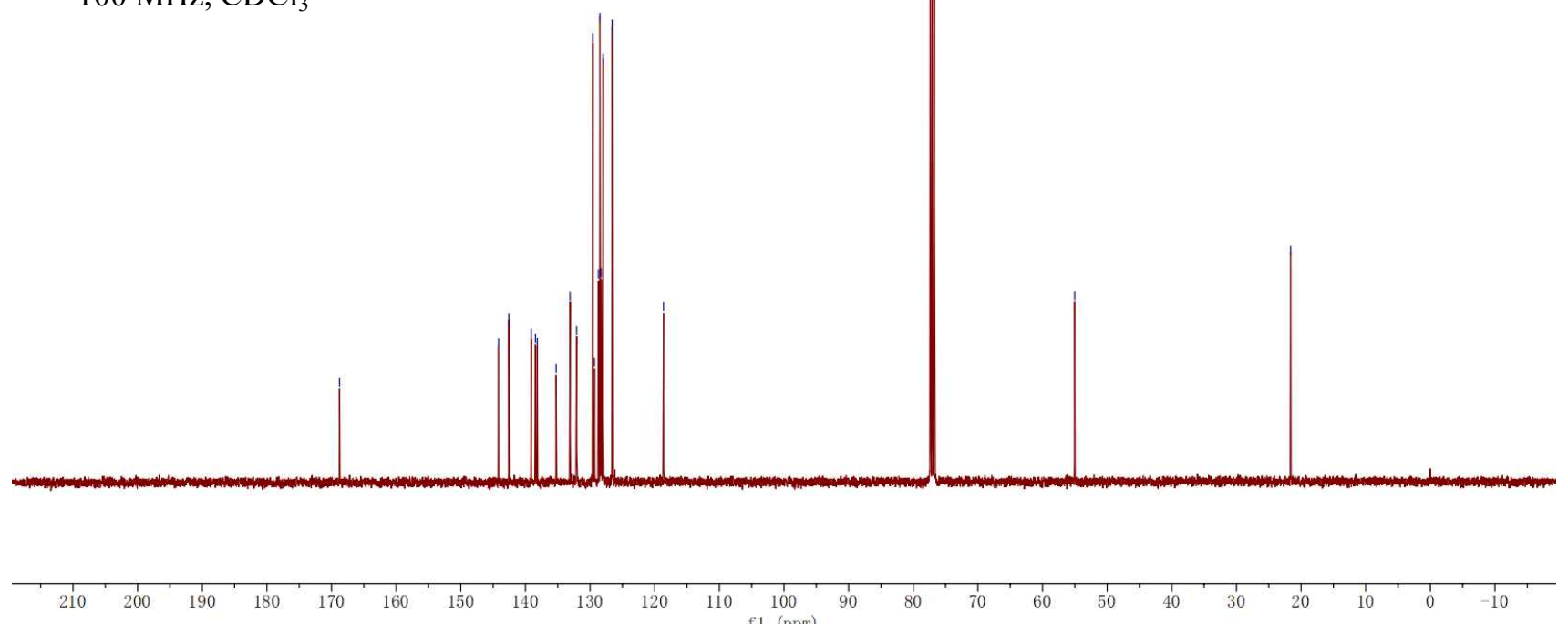
<smiles>C=C(CN(C)c1cc(Br)ccc1C(=O)O)c1ccccc1</smiles>

$1 s$

$400 \mathrm{MHz}, \mathrm{CDCl}_{3}$

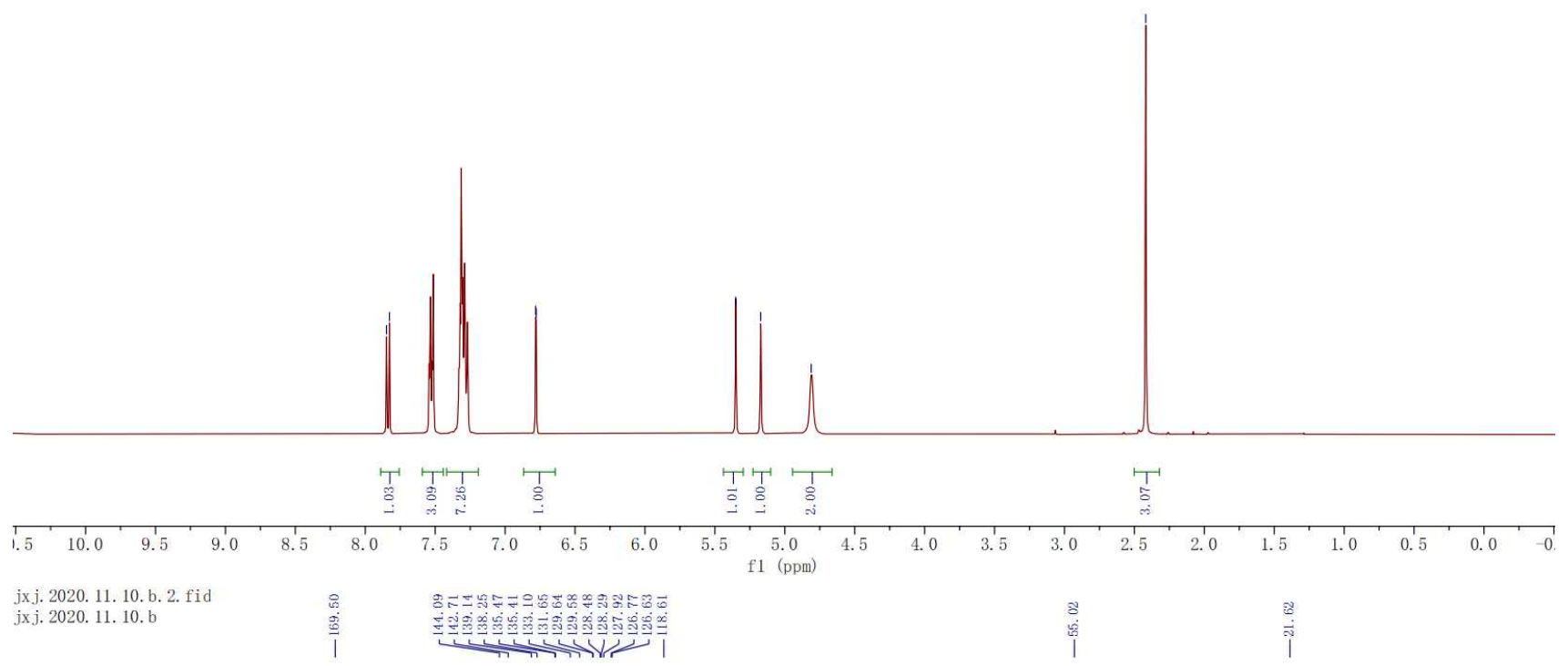<smiles>C=C(CN([As])c1cc(Br)ccc1C(=O)O)c1ccccc1</smiles>

1s

$100 \mathrm{MHz}, \mathrm{CDCl}_{3}$

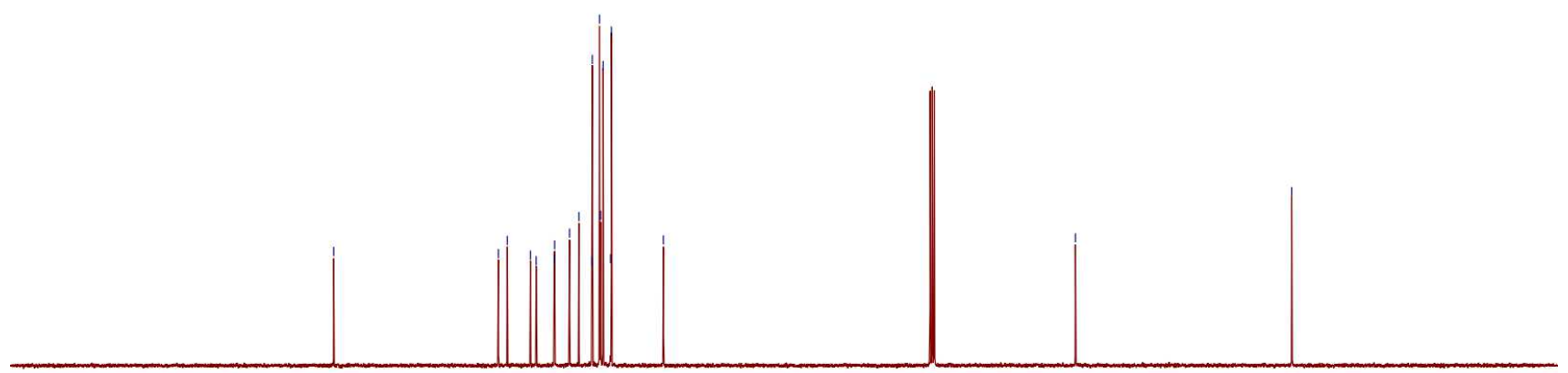

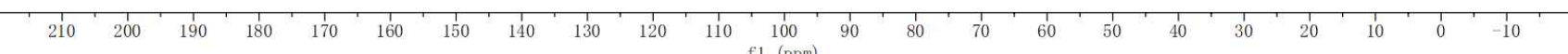




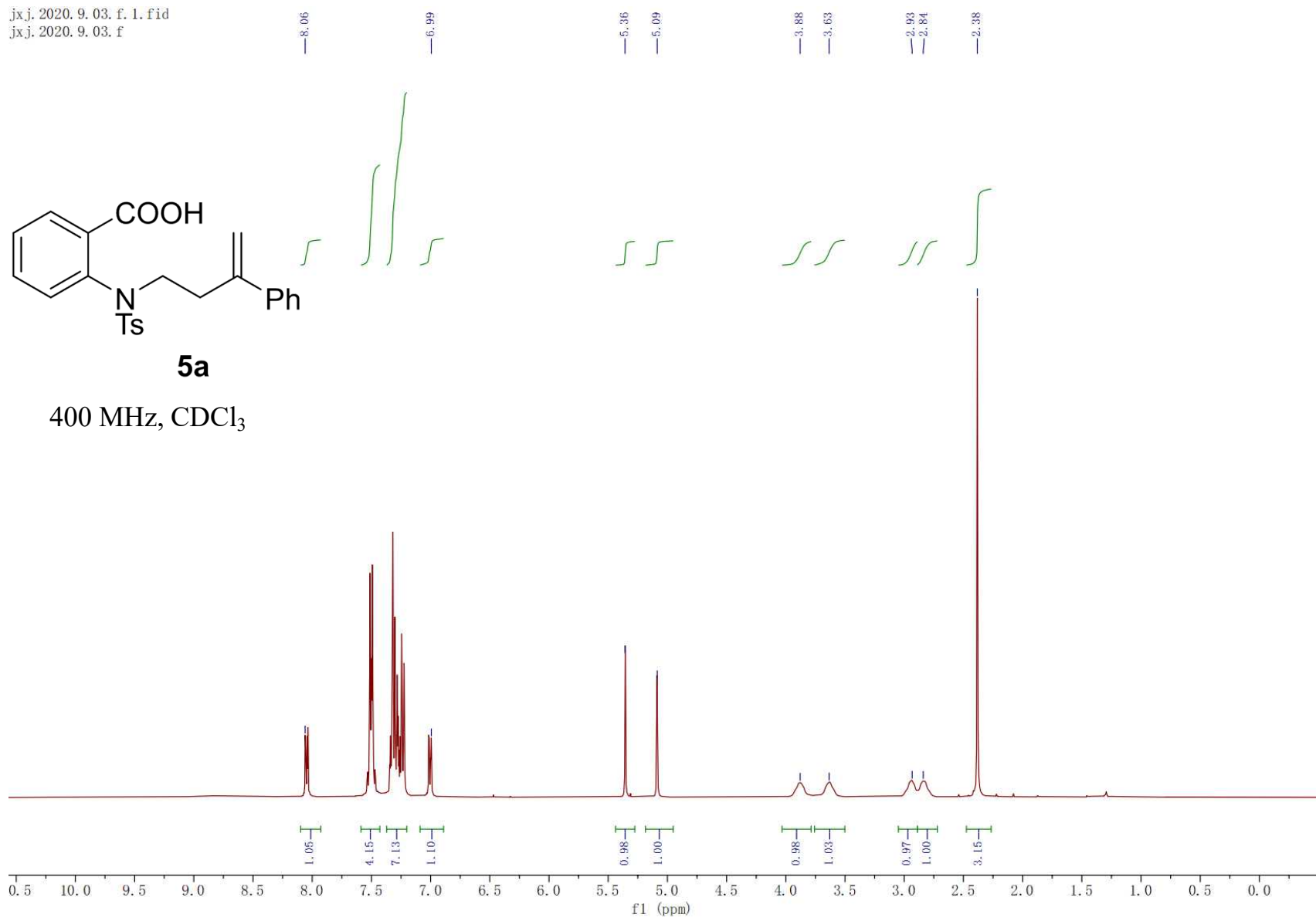

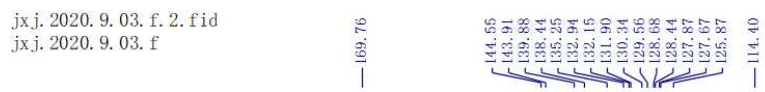<smiles>C=C(CCN([As])c1ccccc1C(=O)O)c1ccccc1</smiles>

$5 a$

$100 \mathrm{MHz}, \mathrm{CDCl}_{3}$

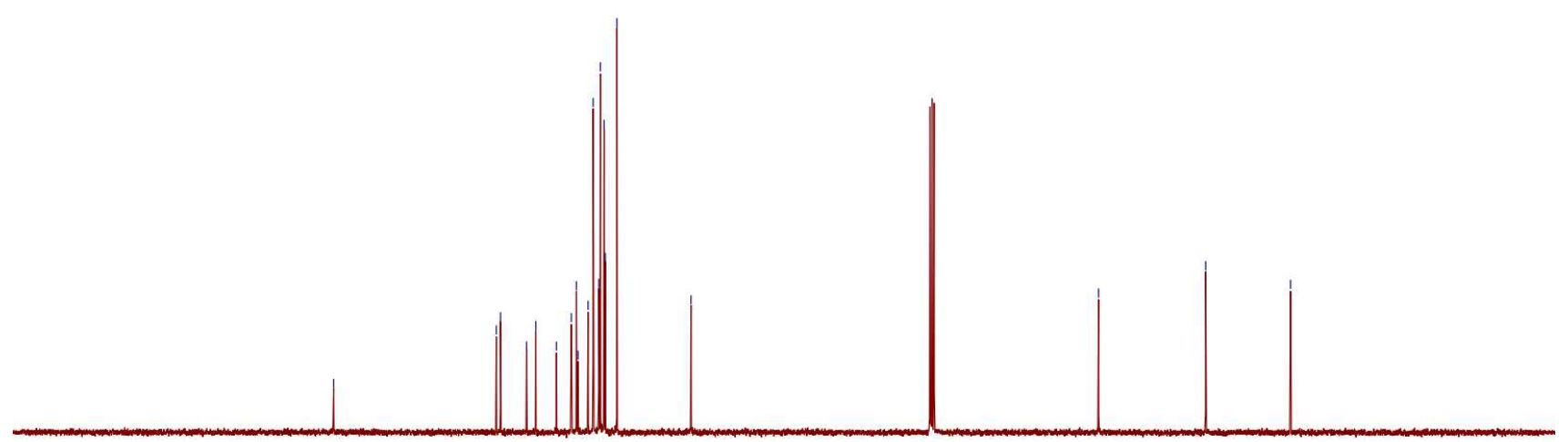

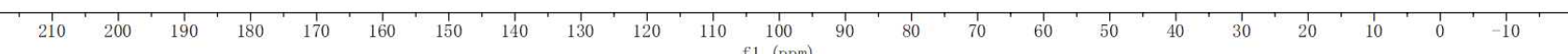




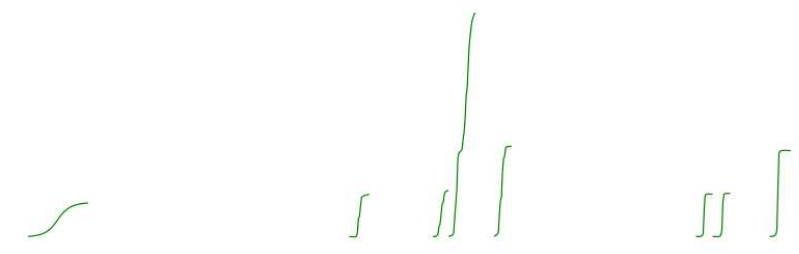<smiles>C=C(COc1ccccc1C(=O)O)c1ccccc1</smiles>

$5 b$

$400 \mathrm{MHz}, \mathrm{CDCl}_{3}$

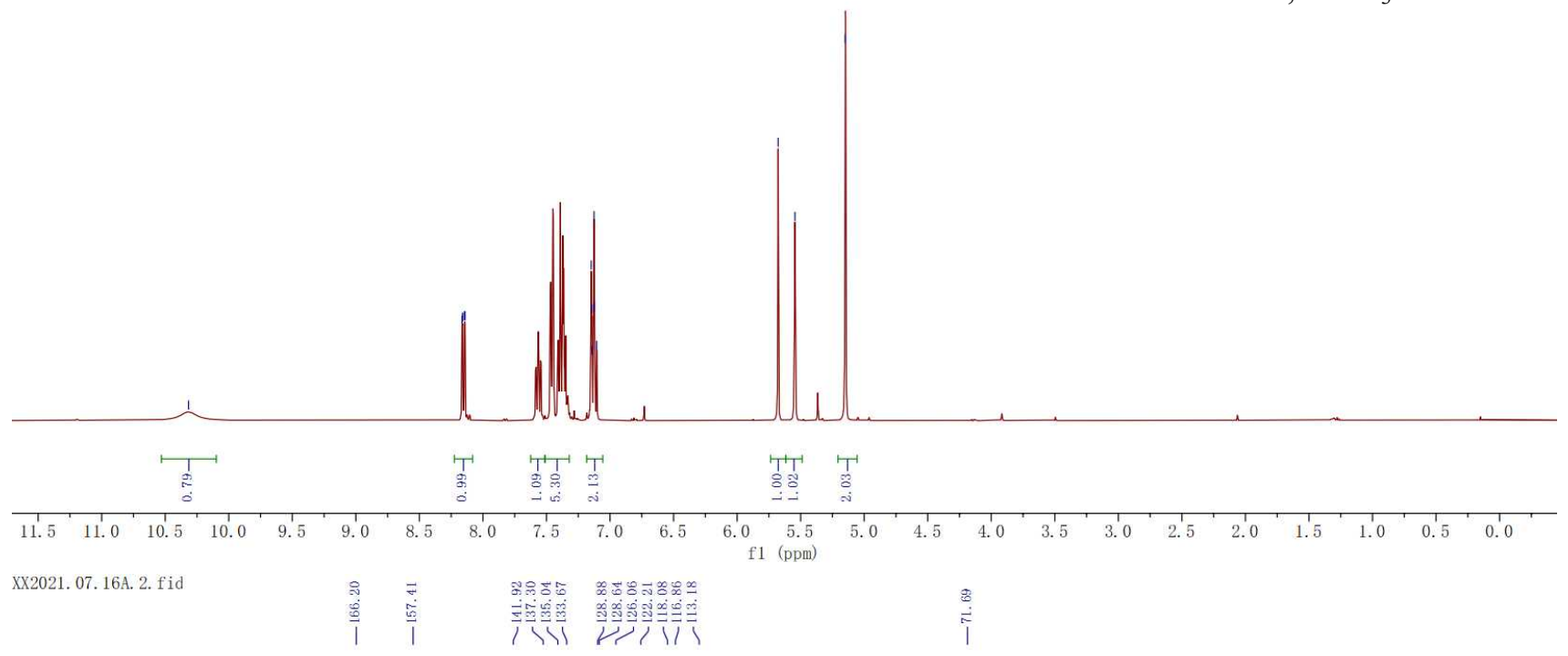<smiles>C=C(COc1ccccc1C(=O)O)c1ccccc1</smiles>

$100 \mathrm{MHz}, \mathrm{CDCl}_{3}$

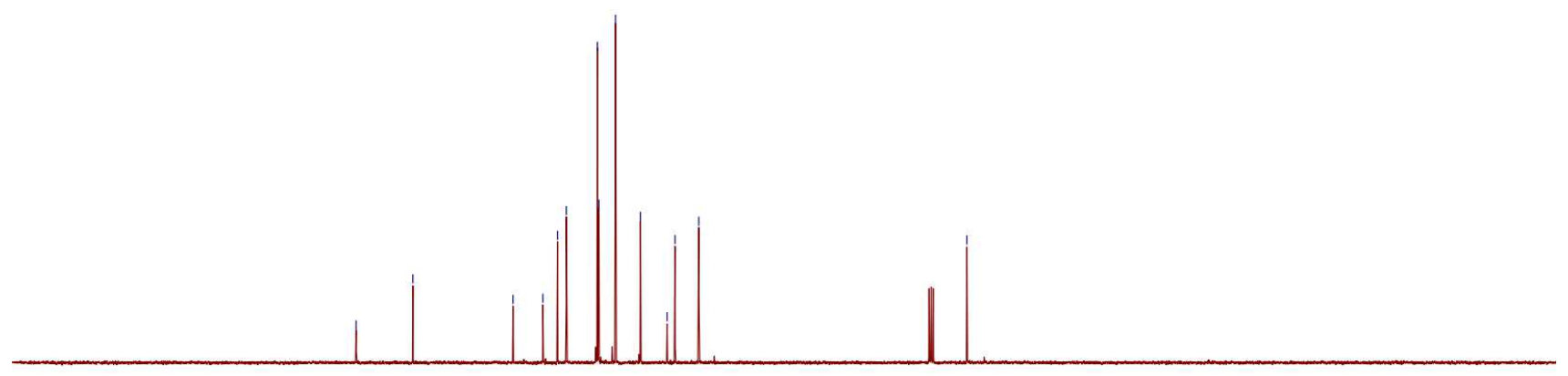

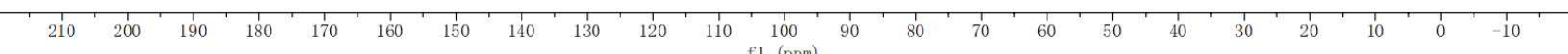



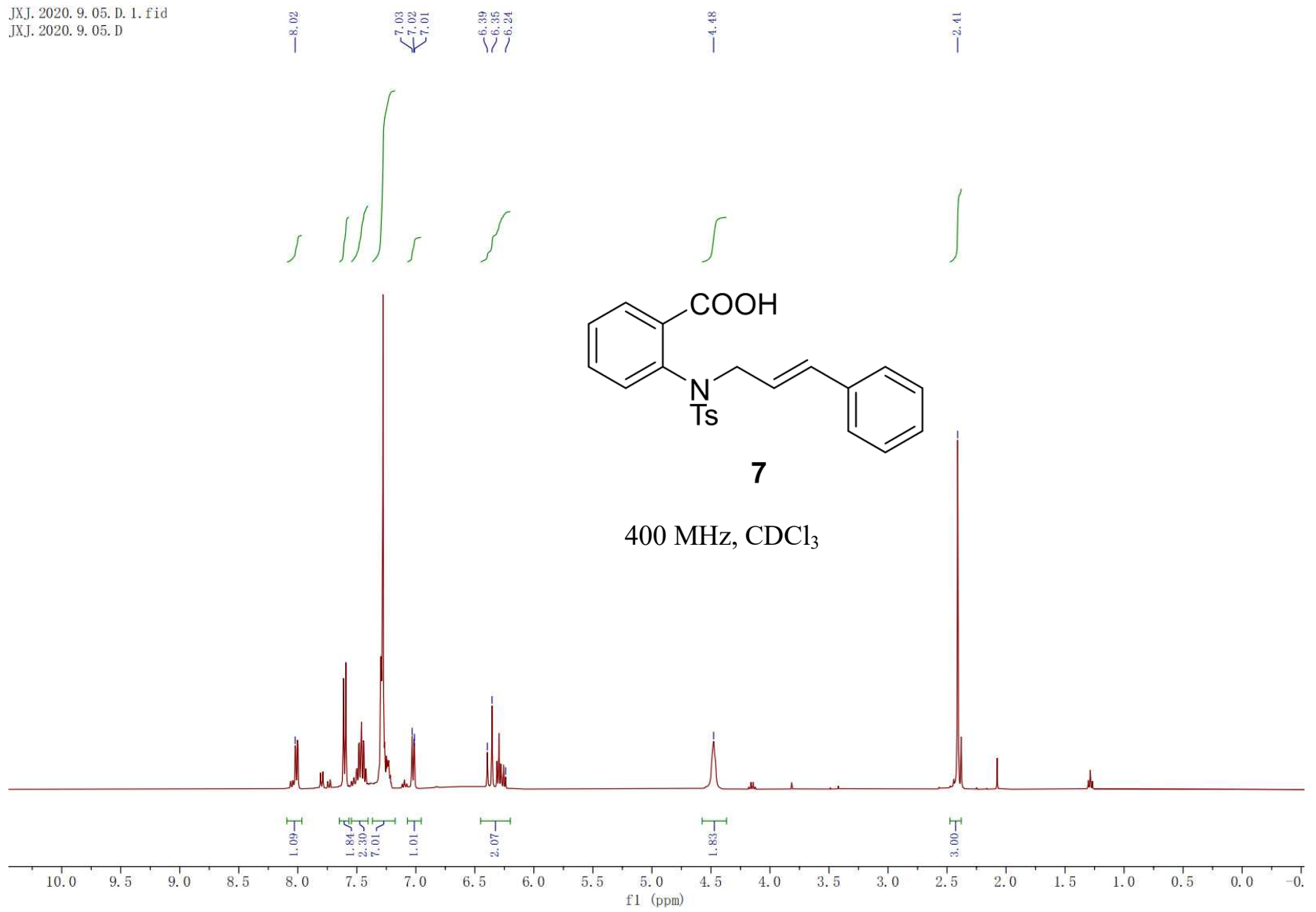

JXJ. 2020. 9. 05. D. 2. fid JXJ. 2020. 9. 05. D

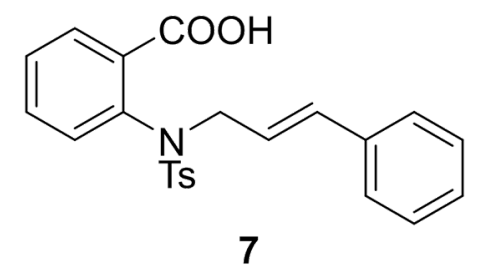

$100 \mathrm{MHz}, \mathrm{CDCl}_{3}$

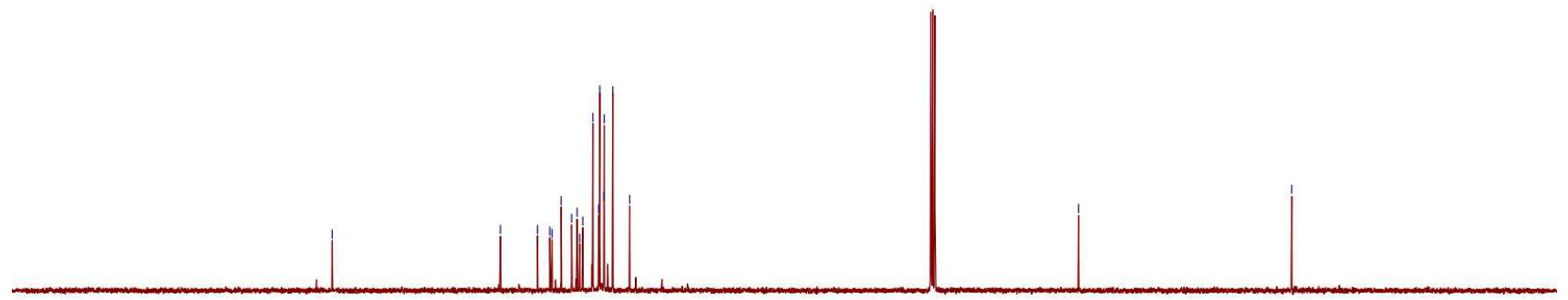

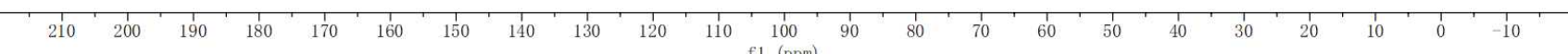


<smiles>O=C1OC(CBr)(c2ccccc2)CN(S)c2ccccc21</smiles><smiles>C=CCCC</smiles>

$2 \mathrm{a}$

$400 \mathrm{MHz}, \mathrm{CDCl}_{3}$

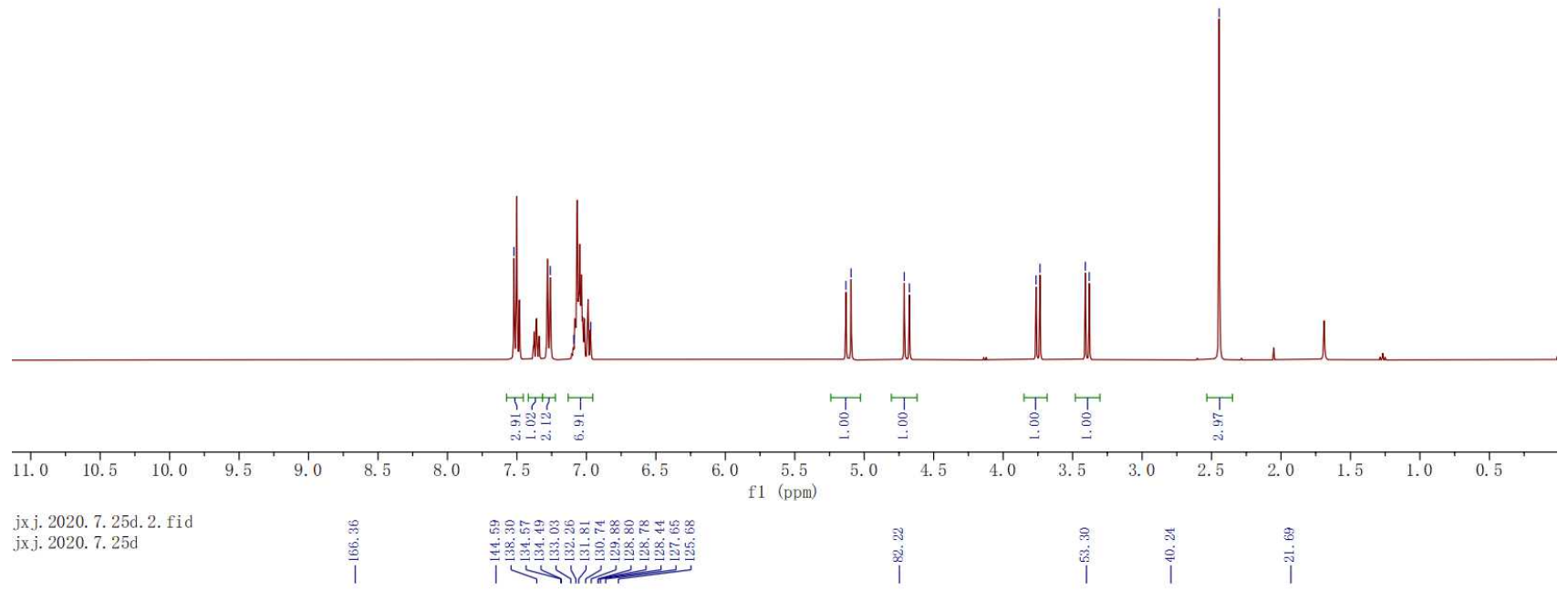<smiles>O=C1O[C@@](CBr)(c2ccccc2)CN([As])c2ccccc21</smiles>

$2 a$

$100 \mathrm{MHz}, \mathrm{CDCl}_{3}$

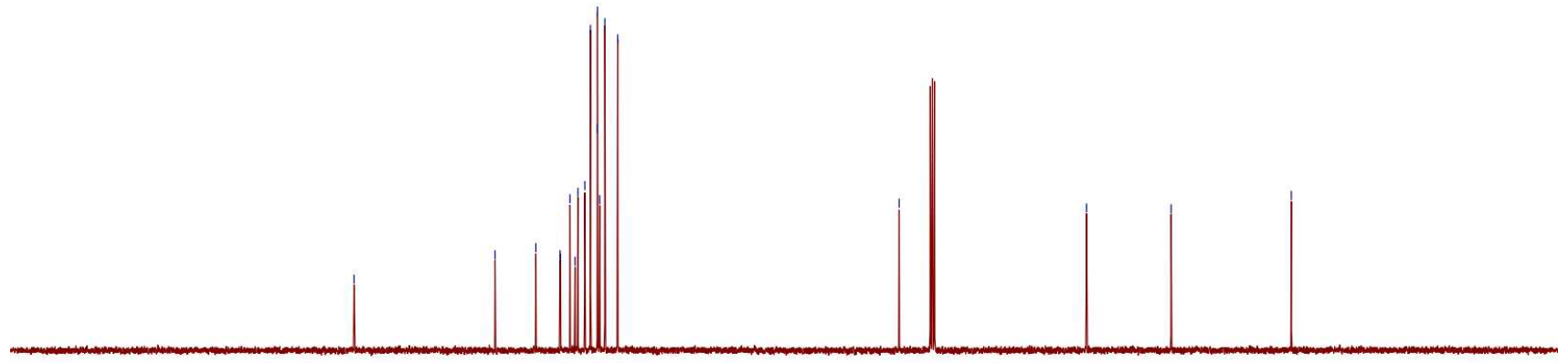

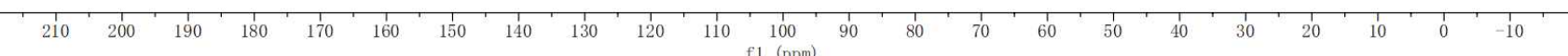


<smiles>O=C1Nc2ccccc2[C@@](CBr)(c2ccccc2)O1</smiles>

2b

$400 \mathrm{MHz}, \mathrm{CDCl}_{3}$

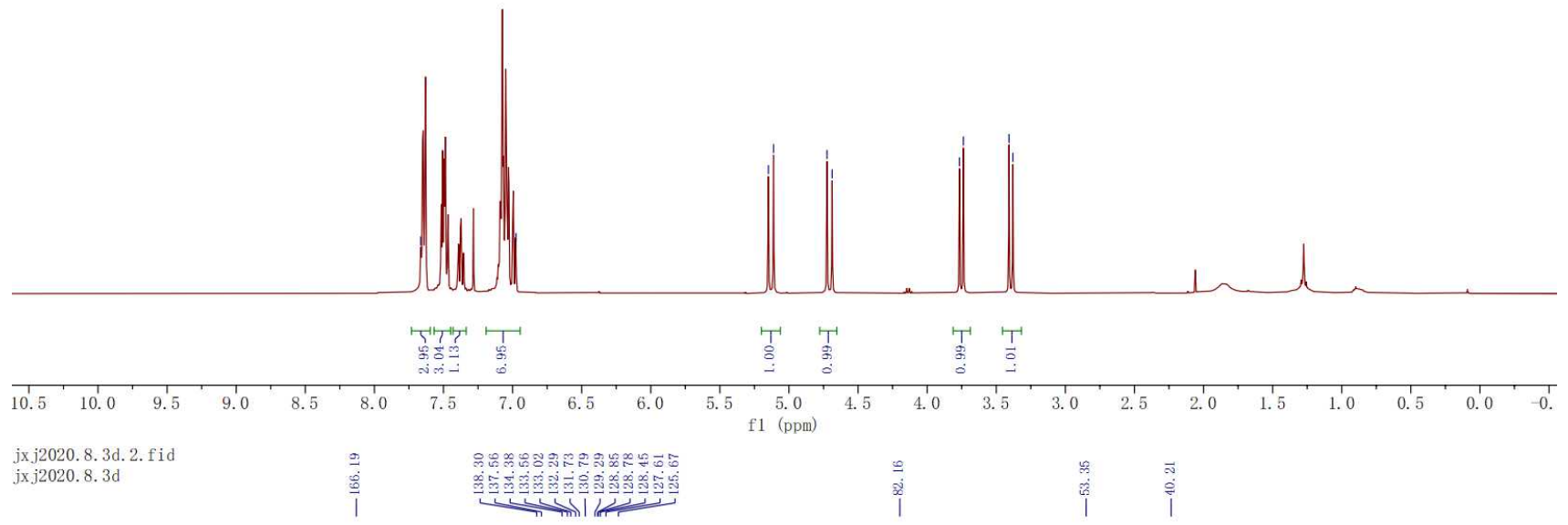<smiles>O=C1O[C@](CBr)(c2ccccc2)CNc2ccccc21</smiles>

Bs

2b

$100 \mathrm{MHz}, \mathrm{CDCl}_{3}$

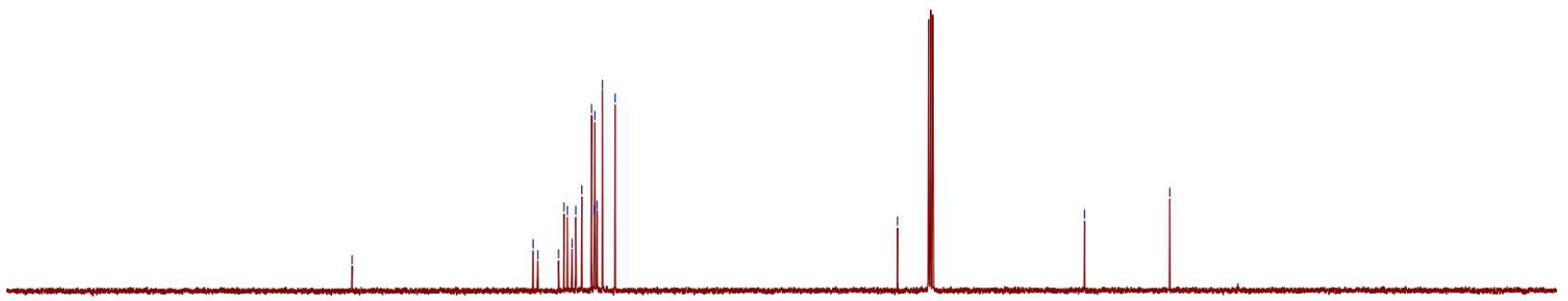

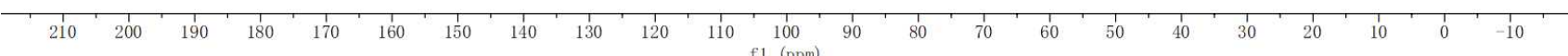


<smiles>COc1ccc(S(=O)(=O)N2C[C@](CBr)(c3ccccc3)OC(=O)c3ccccc32)cc1</smiles>

$400 \mathrm{MHz}, \mathrm{CDCl}_{3}$

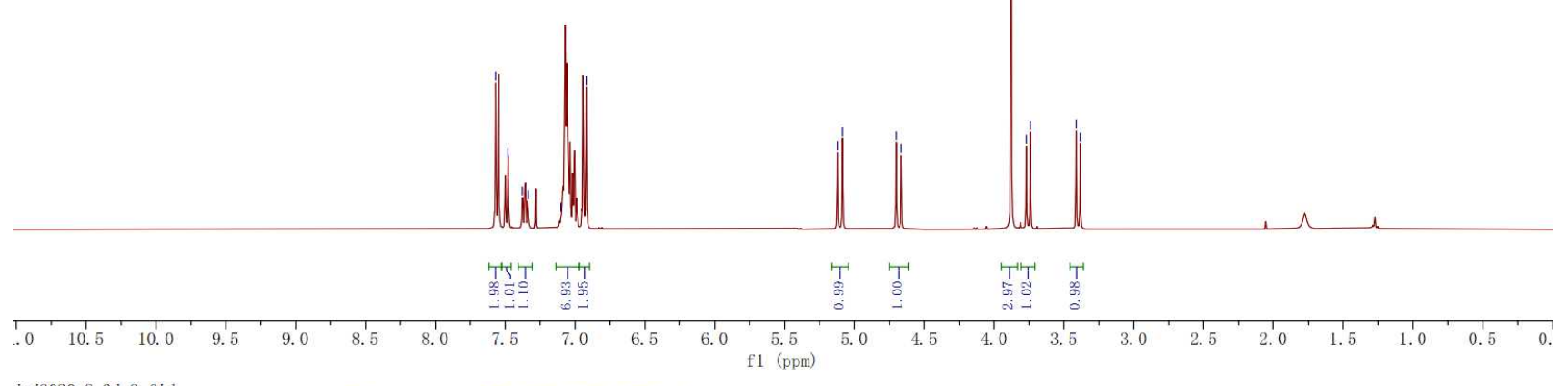<smiles>COc1ccc(S(=O)(=O)N2CC(CBr)(c3ccccc3)OC(=O)c3ccccc32)cc1</smiles>

$100 \mathrm{MHz}, \mathrm{CDCl}_{3}$

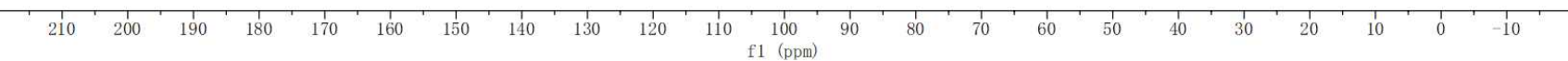



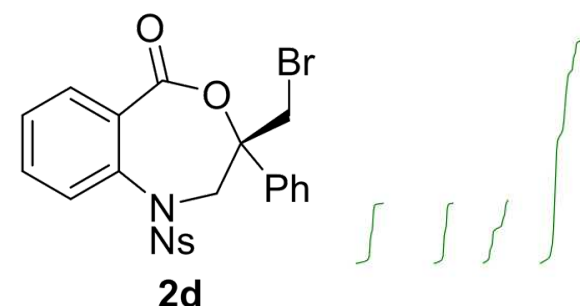

$400 \mathrm{MHz}, \mathrm{CDCl}_{3}$

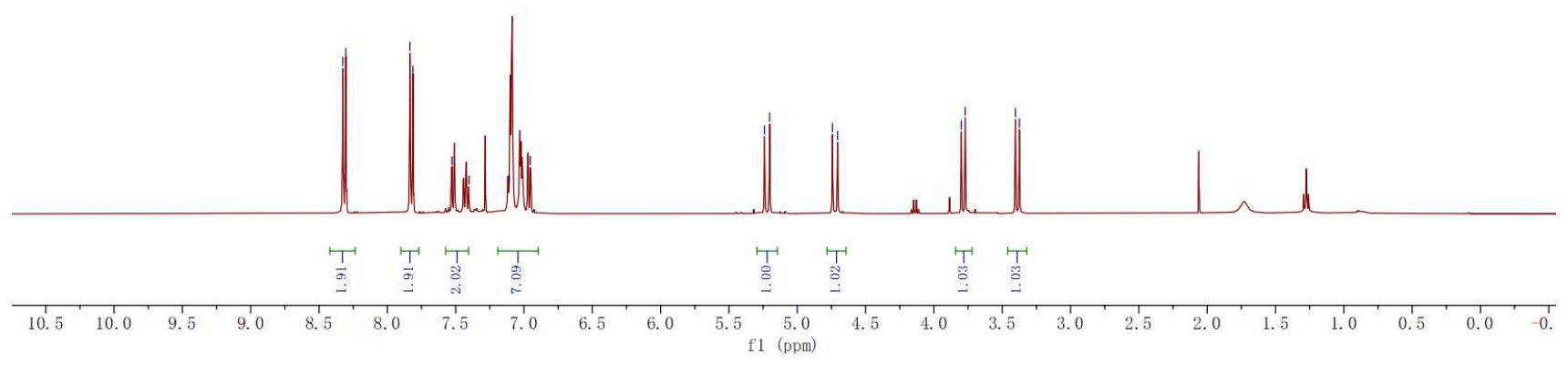

JXJ. 2020. 8. 9. D.2. fid

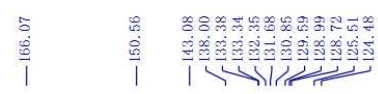

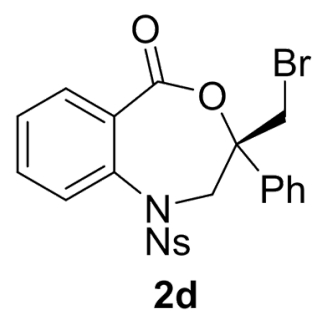

$100 \mathrm{MHz}, \mathrm{CDCl}_{3}$

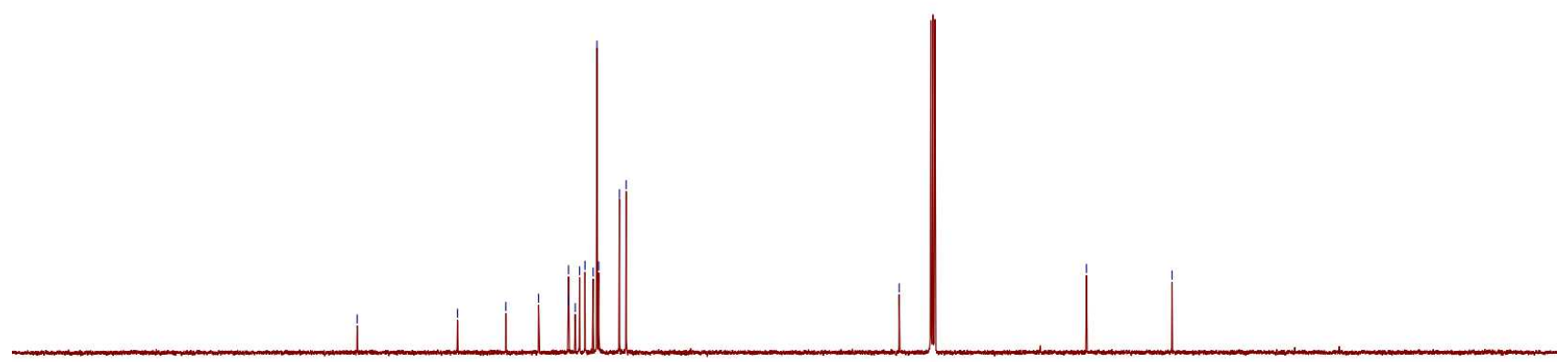

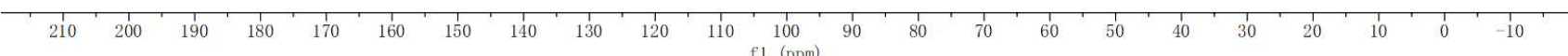


<smiles>CS(=O)(=O)c1ccccc1C(=O)OC(CBr)(CBr)c1ccccc1</smiles><smiles>C1CCCC1</smiles>

$2 e$

$400 \mathrm{MHz}, \mathrm{CDCl}_{3}$

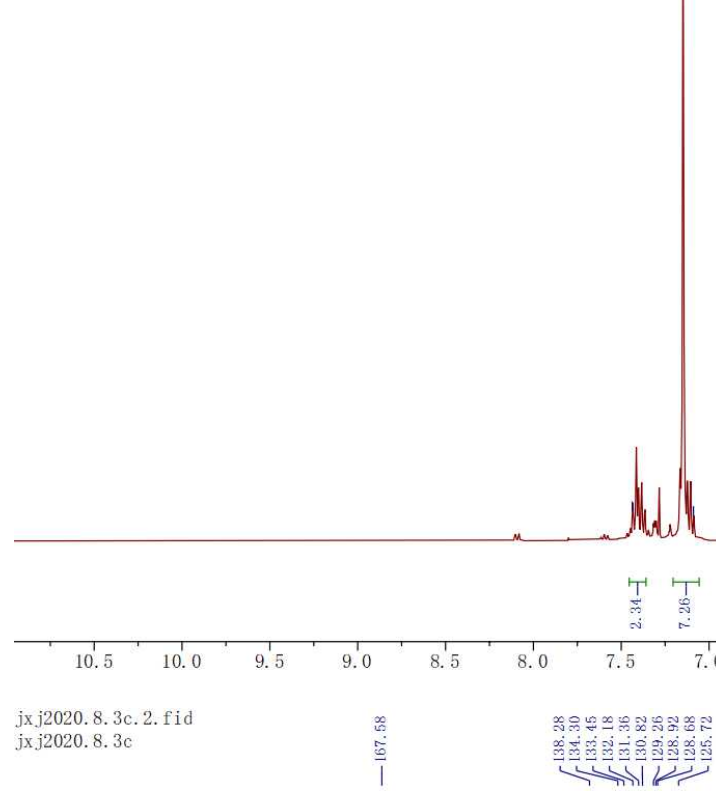<smiles>Cc1ccccc1C(=O)OC(CBr)(CBr)c1ccccc1</smiles>

$2 e$

$100 \mathrm{MHz}, \mathrm{CDCl}_{3}$

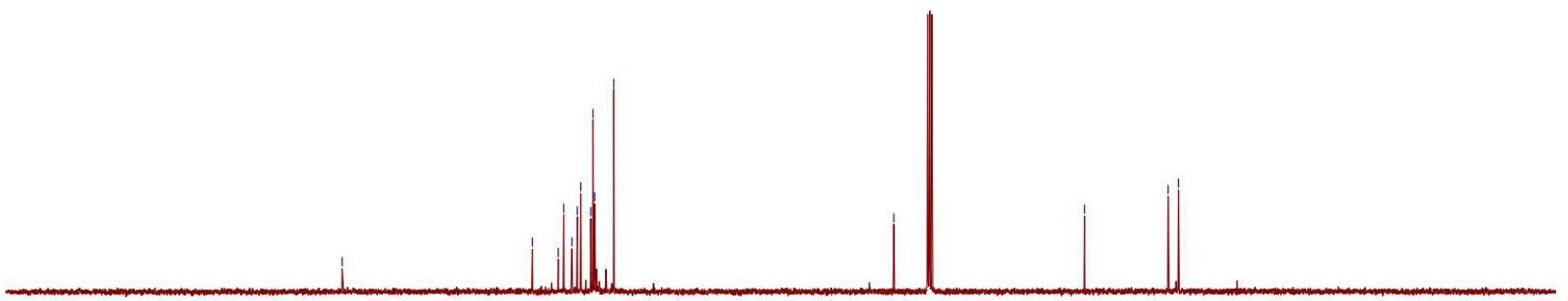




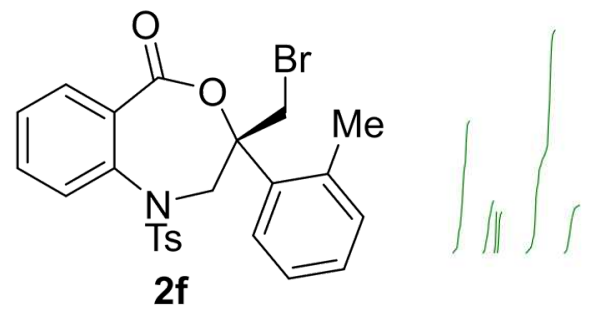

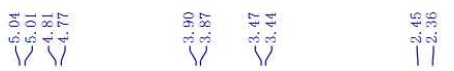

$400 \mathrm{MHz}, \mathrm{CDCl}_{3}$

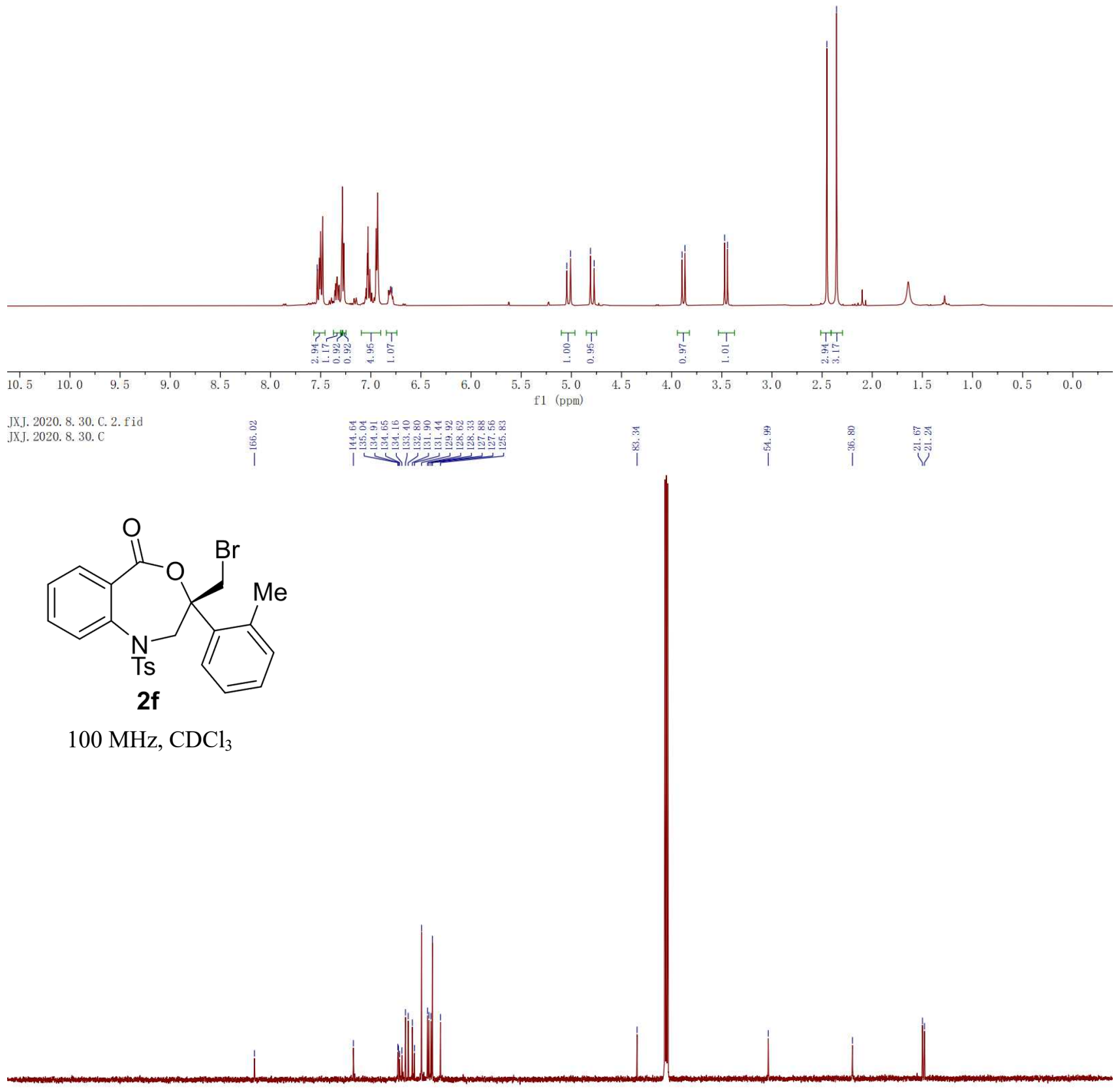

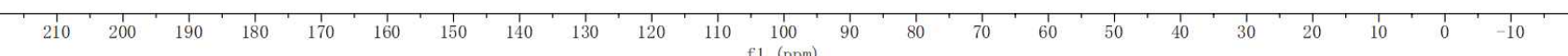




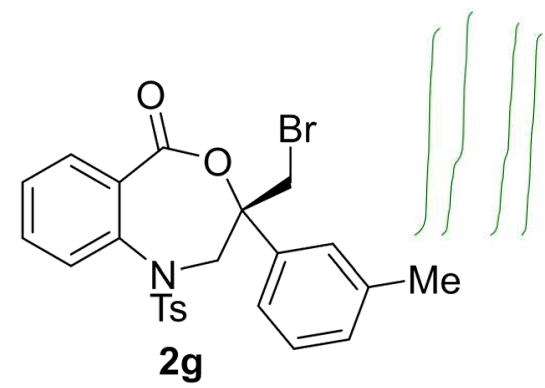

$400 \mathrm{MHz}, \mathrm{CDCl}_{3}$
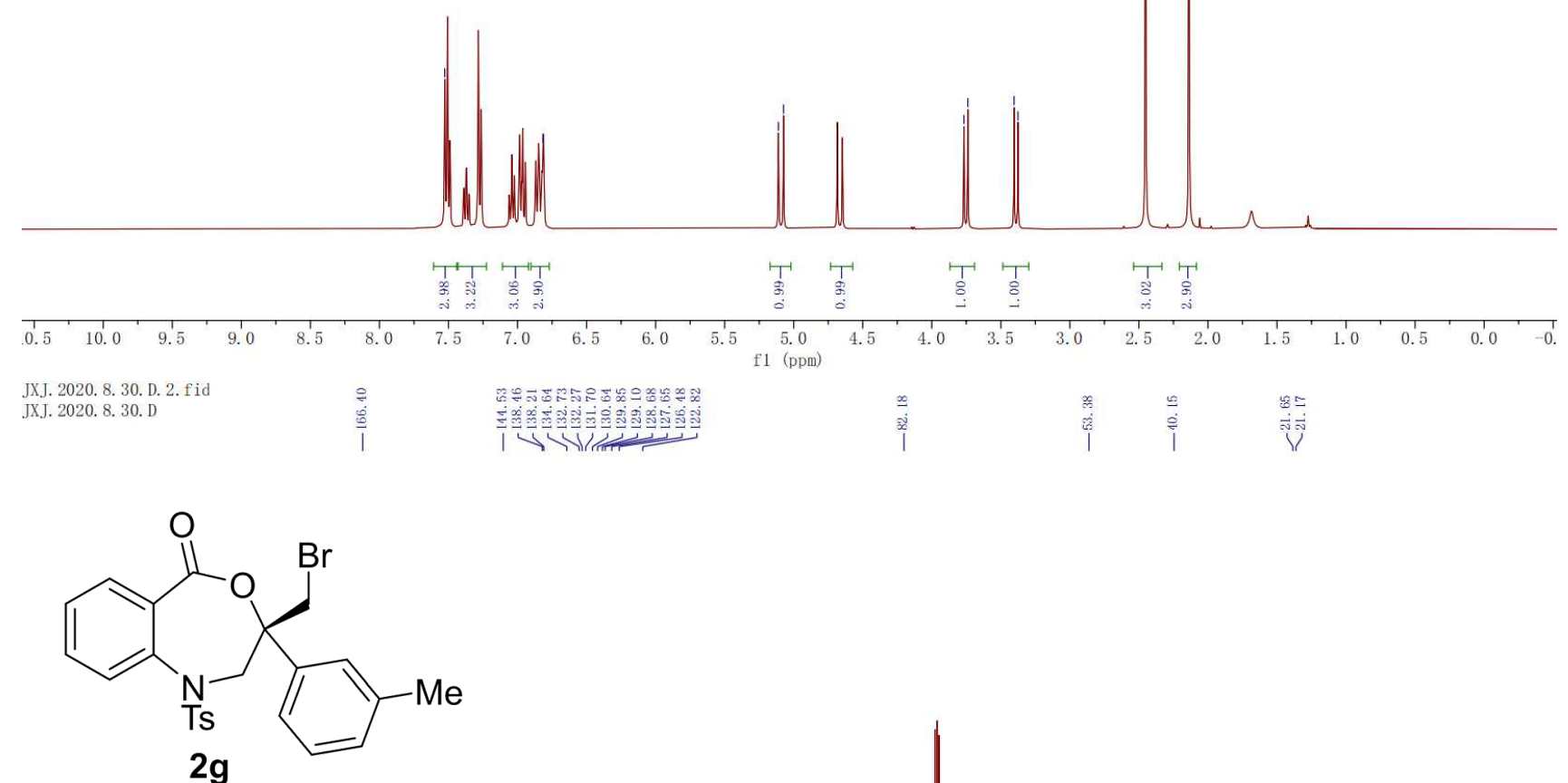

$100 \mathrm{MHz}, \mathrm{CDCl}_{3}$

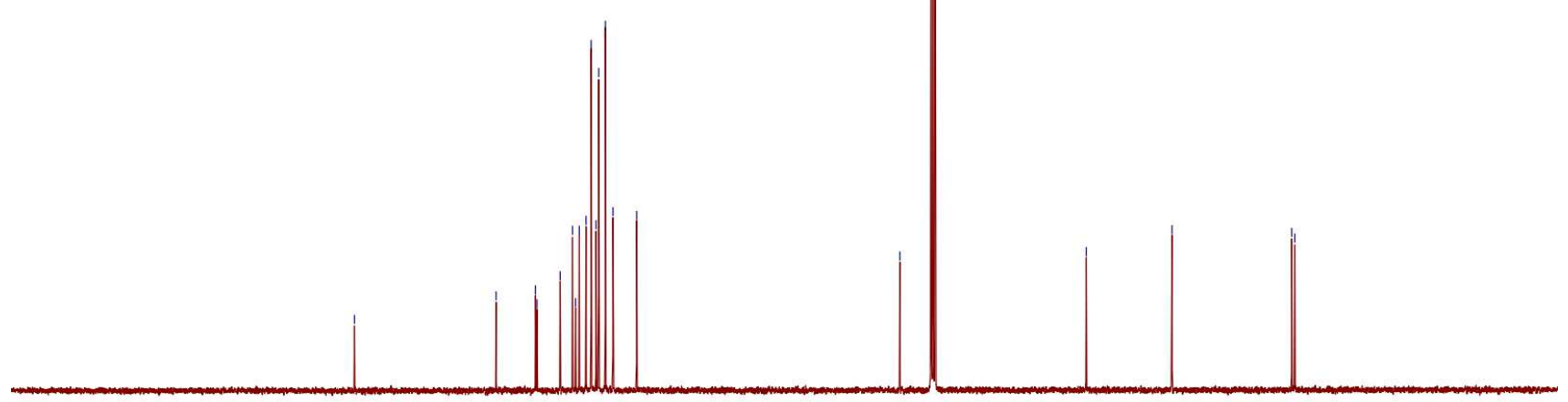

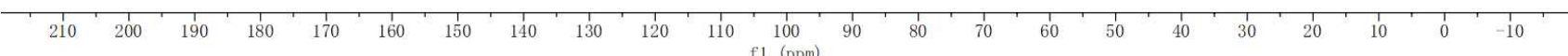


<smiles>[R6]N1C[C@@](CBr)(c2ccc(C)cc2)OC(=O)c2ccccc21</smiles>

$400 \mathrm{MHz}, \mathrm{CDCl}_{3}$

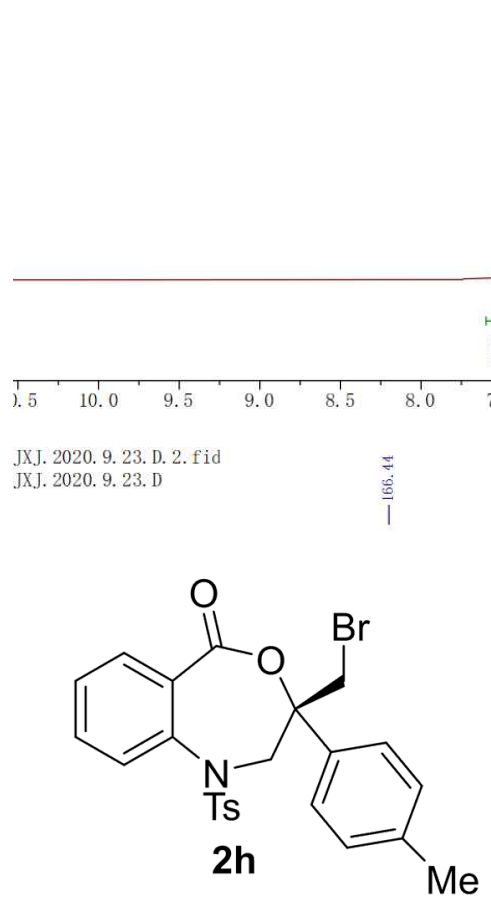

$100 \mathrm{MHz}, \mathrm{CDCl}_{3}$

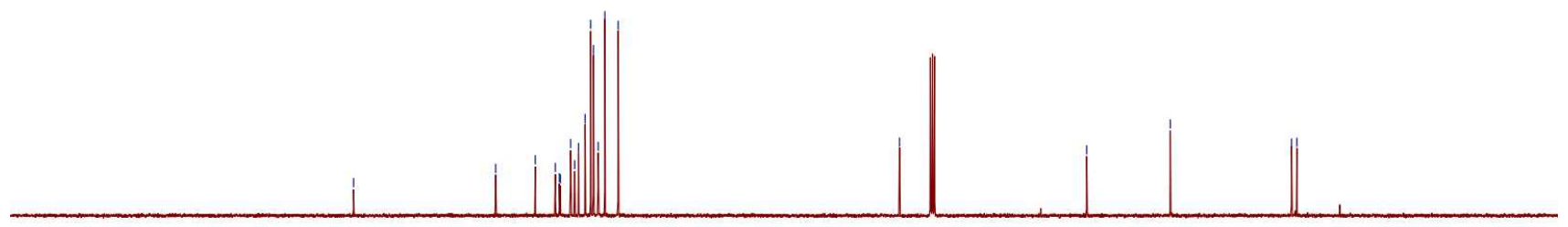

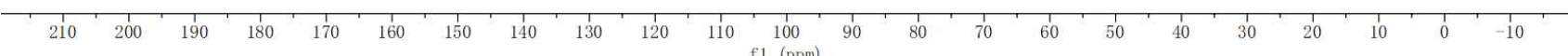




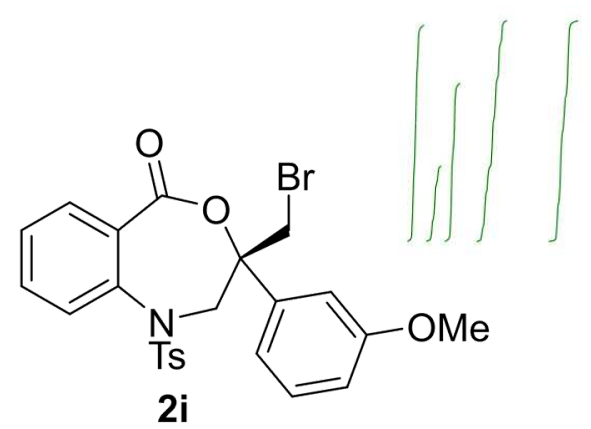

$400 \mathrm{MHz}, \mathrm{CDCl}_{3}$
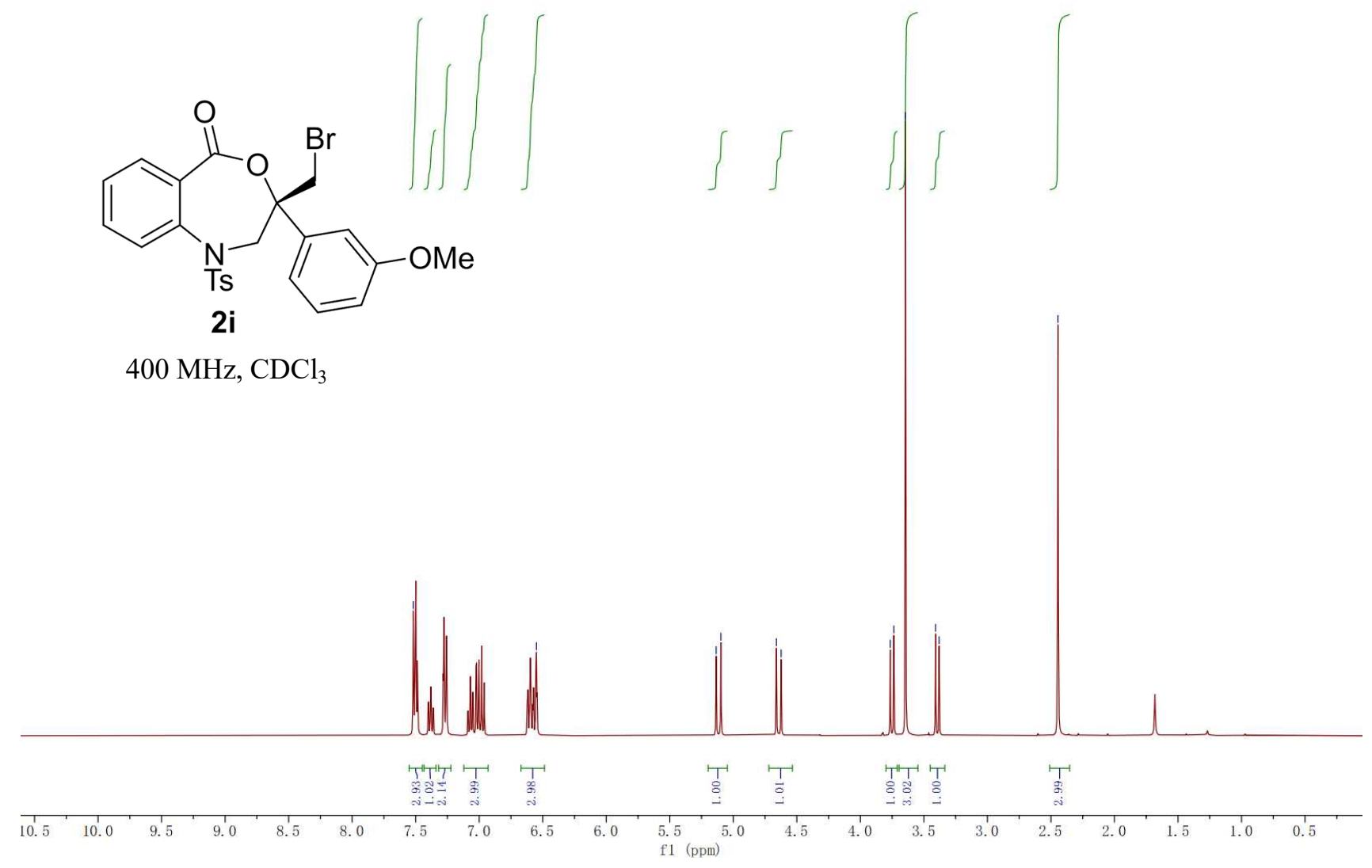

\section{jx j. 2020. 10. 03. a. 2. fid

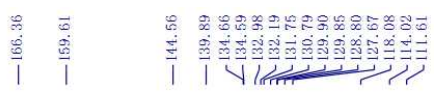

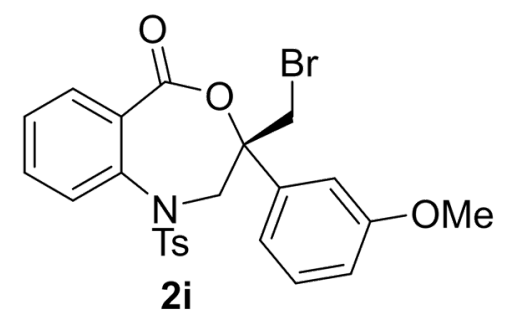

$100 \mathrm{MHz}, \mathrm{CDCl}_{3}$

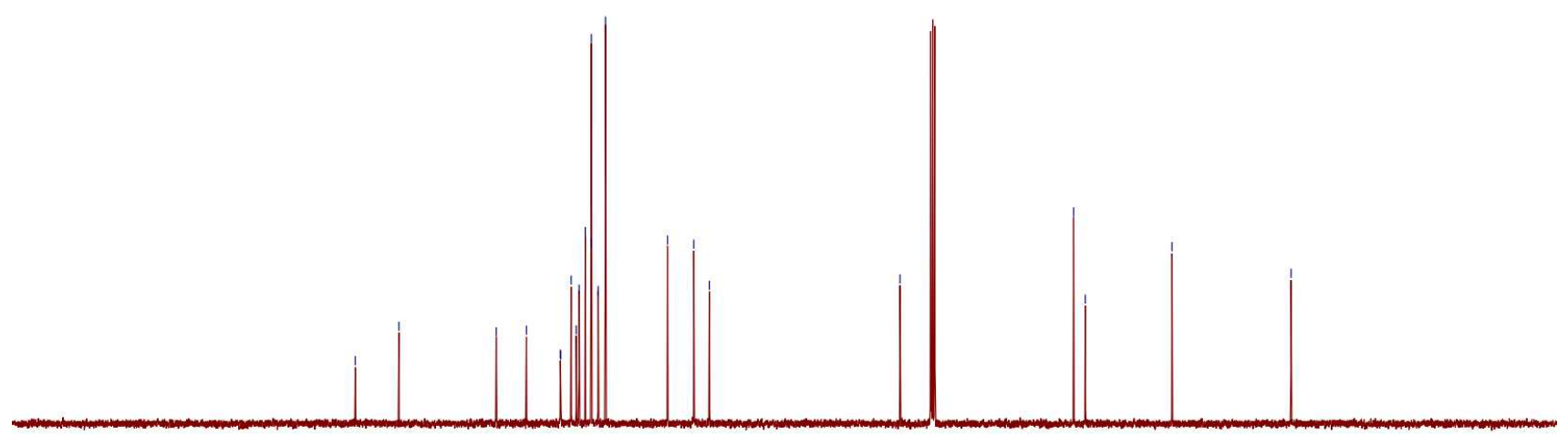

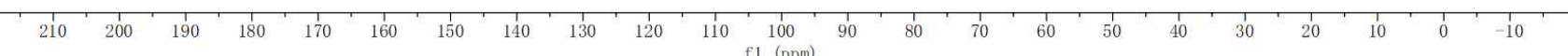


<smiles>O=C1O[C@@](CBr)(c2cccc(Br)c2)CN([As])c2ccccc21</smiles>

$400 \mathrm{MHz}, \mathrm{CDCl}_{3}$
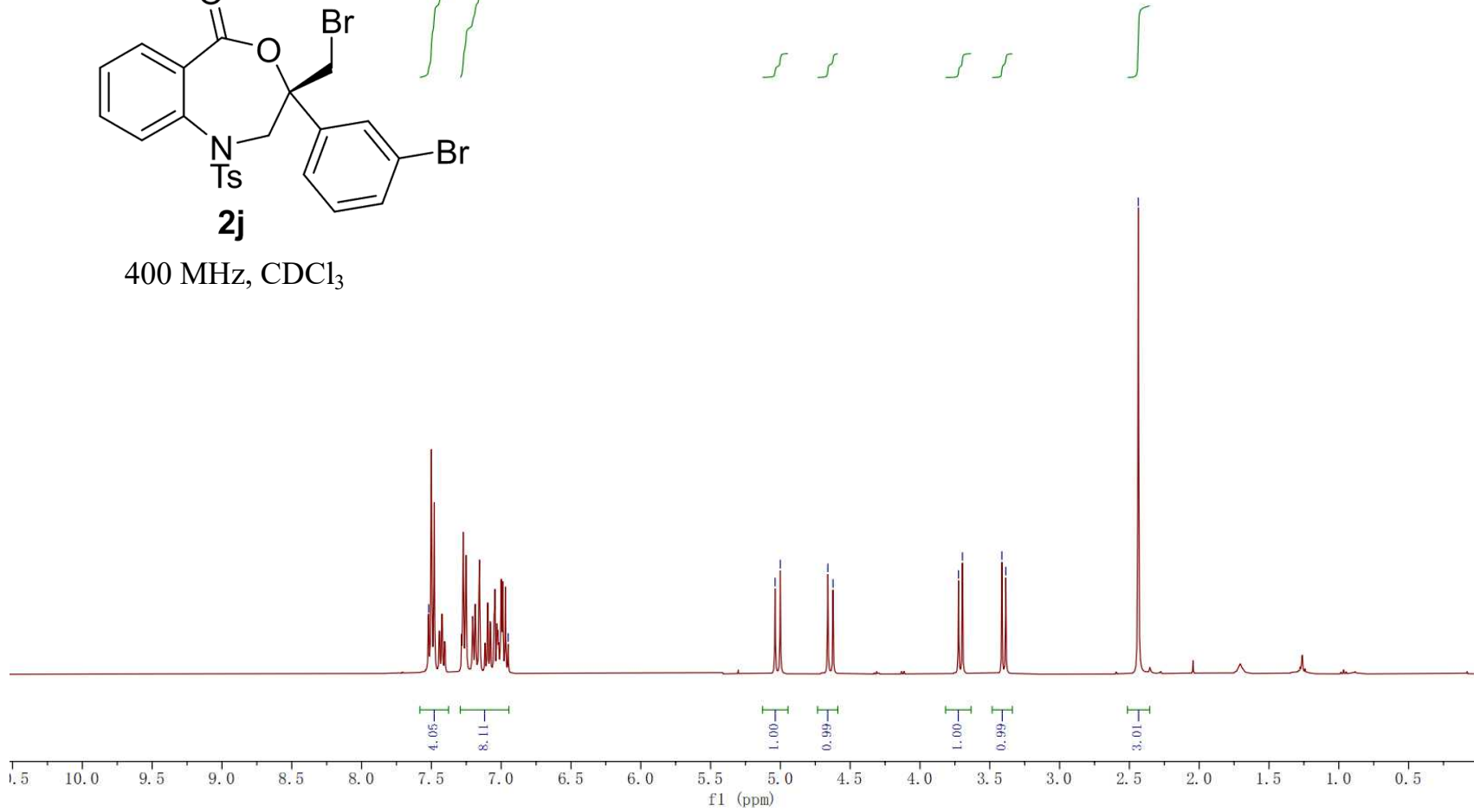

JXJ. 2020. 9. 23. E. 2. fid
JXJ. 2020. 9. 23. E
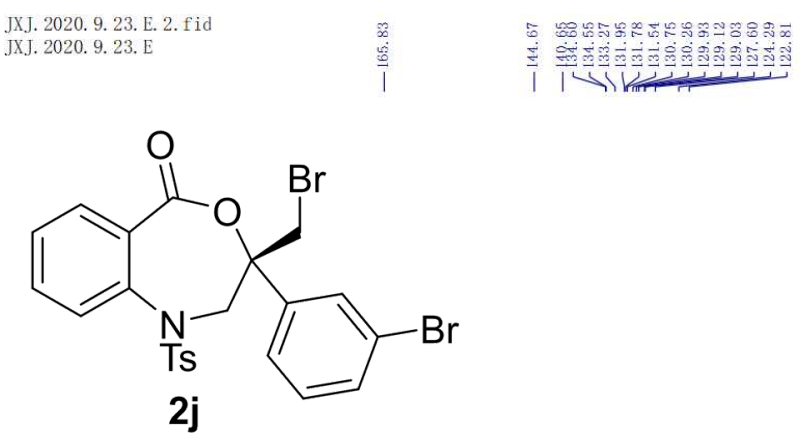

$100 \mathrm{MHz}, \mathrm{CDCl}_{3}$

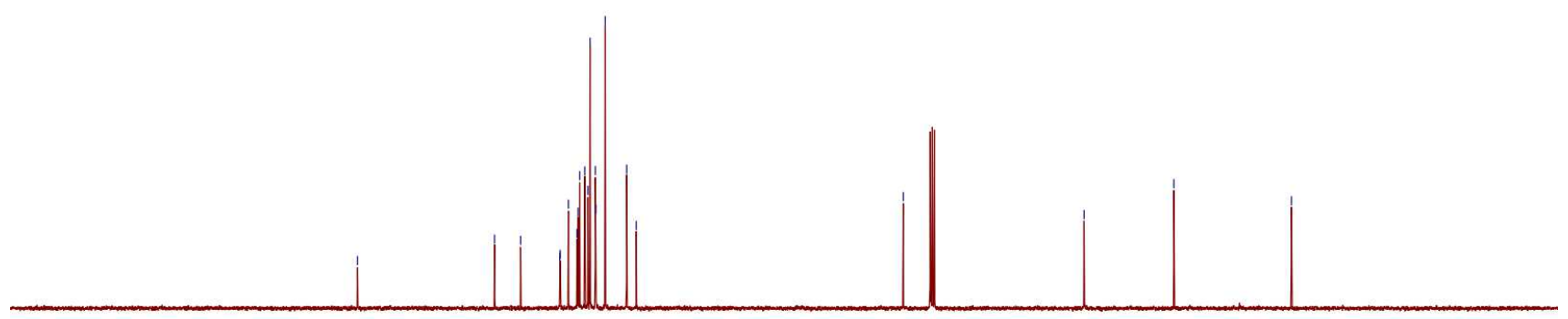

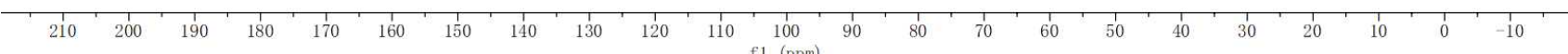


<smiles>[R16]N1C[C@@](CBr)(c2cccc3ccccc23)OC(=O)c2ccccc21</smiles>

$400 \mathrm{MHz}, \mathrm{CDCl}_{3}$

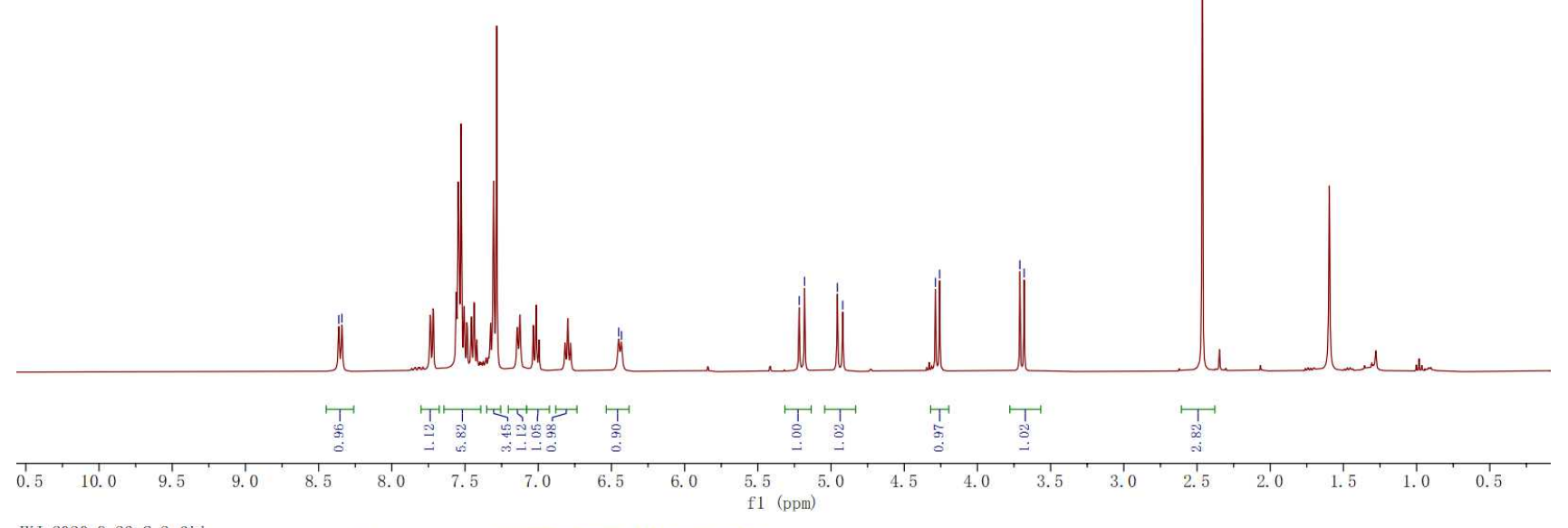
JXJ. 2020. 9. 23. C. 2. fid JXJ. 2020. 9. 23. C

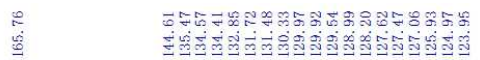

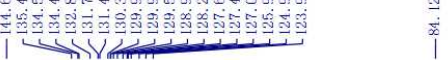<smiles>[R19]N1C[C@@](CBr)(c2cccc3ccccc23)OC(=O)c2ccccc21</smiles>

$100 \mathrm{MHz}, \mathrm{CDCl}_{3}$

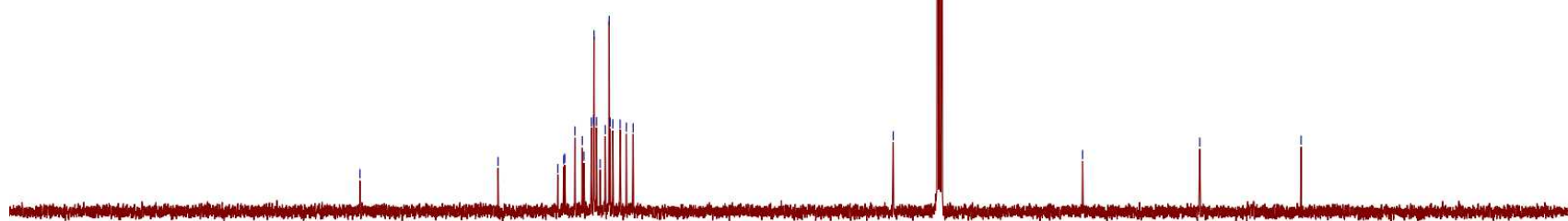




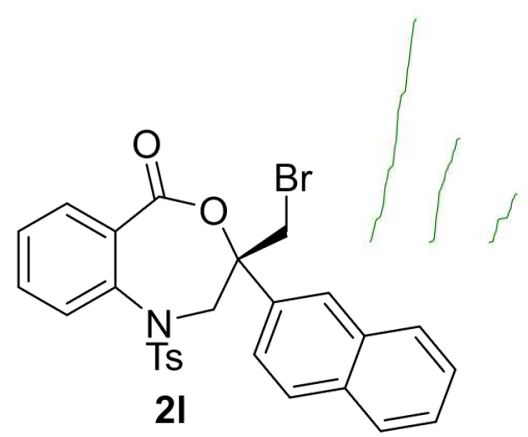

$400 \mathrm{MHz}, \mathrm{CDCl}_{3}$

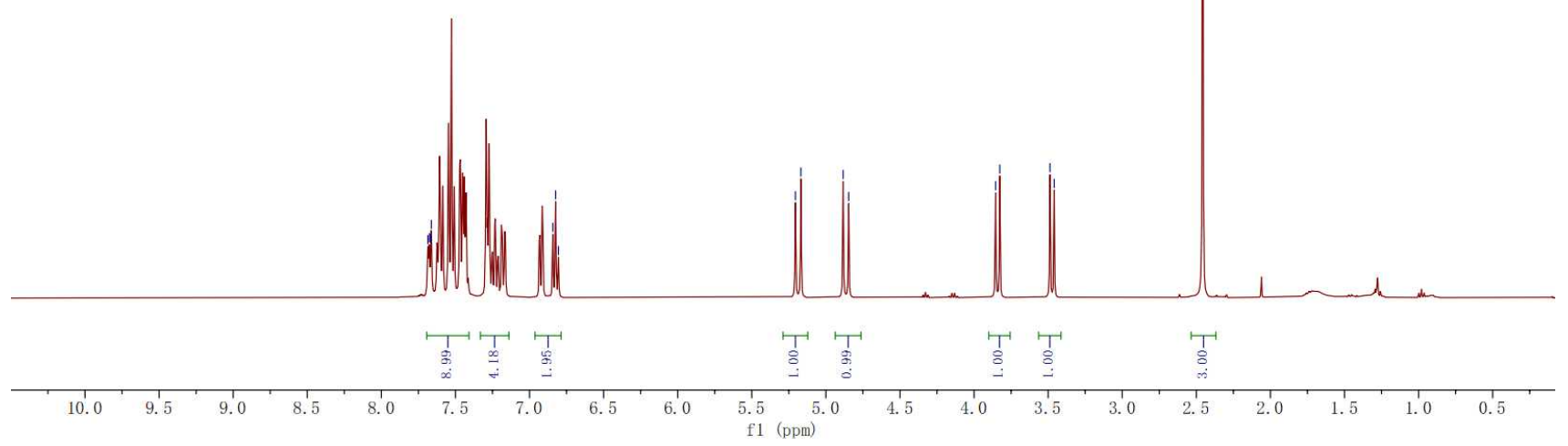

jx j. 2020. 10. 03.c. 2. fid

jx j. 2020. 10. 03. c
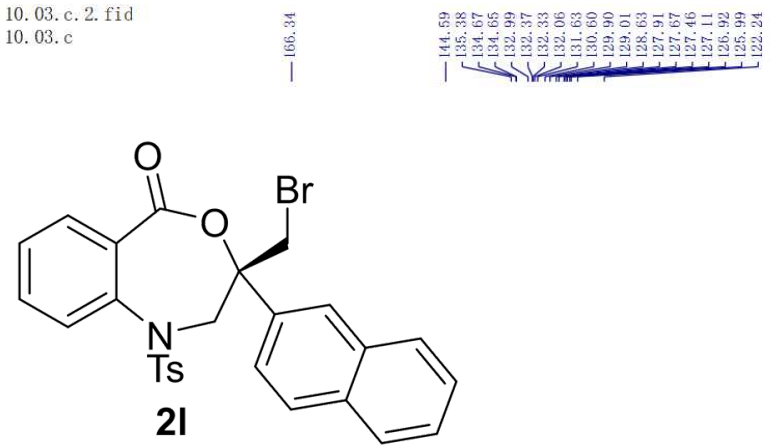

$100 \mathrm{MHz}, \mathrm{CDCl}_{3}$

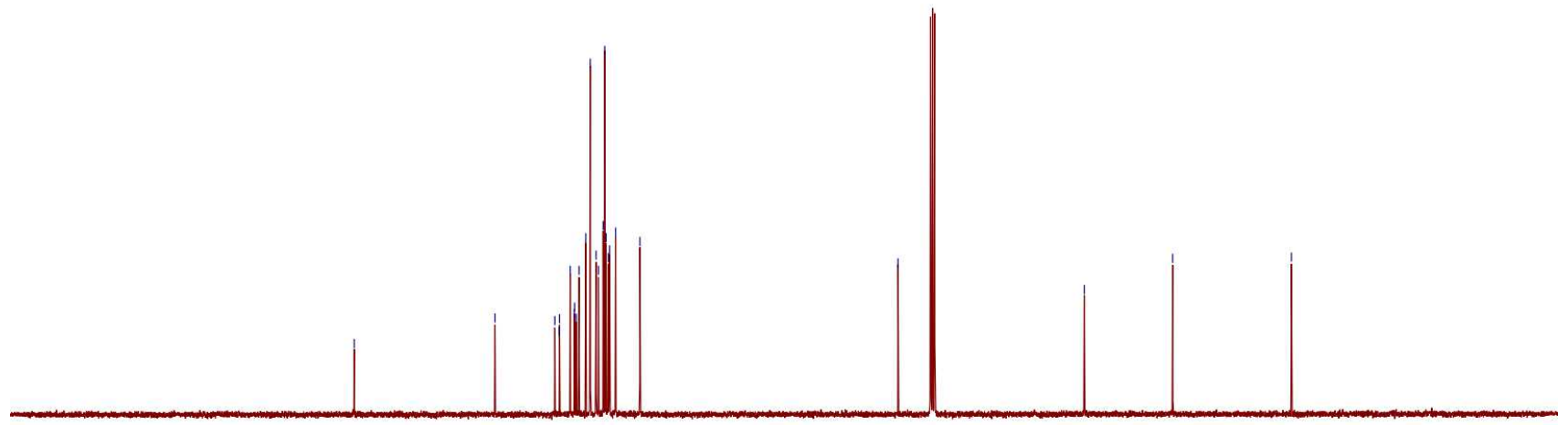

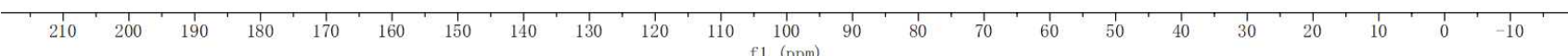


<smiles>Cc1cccc2c1N(C)C[C@](CBr)(c1ccccc1)OC2=O</smiles>

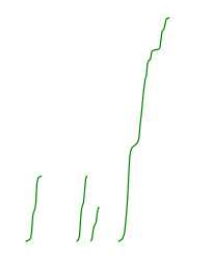
$400 \mathrm{MHz}, \mathrm{CDCl}_{3}$

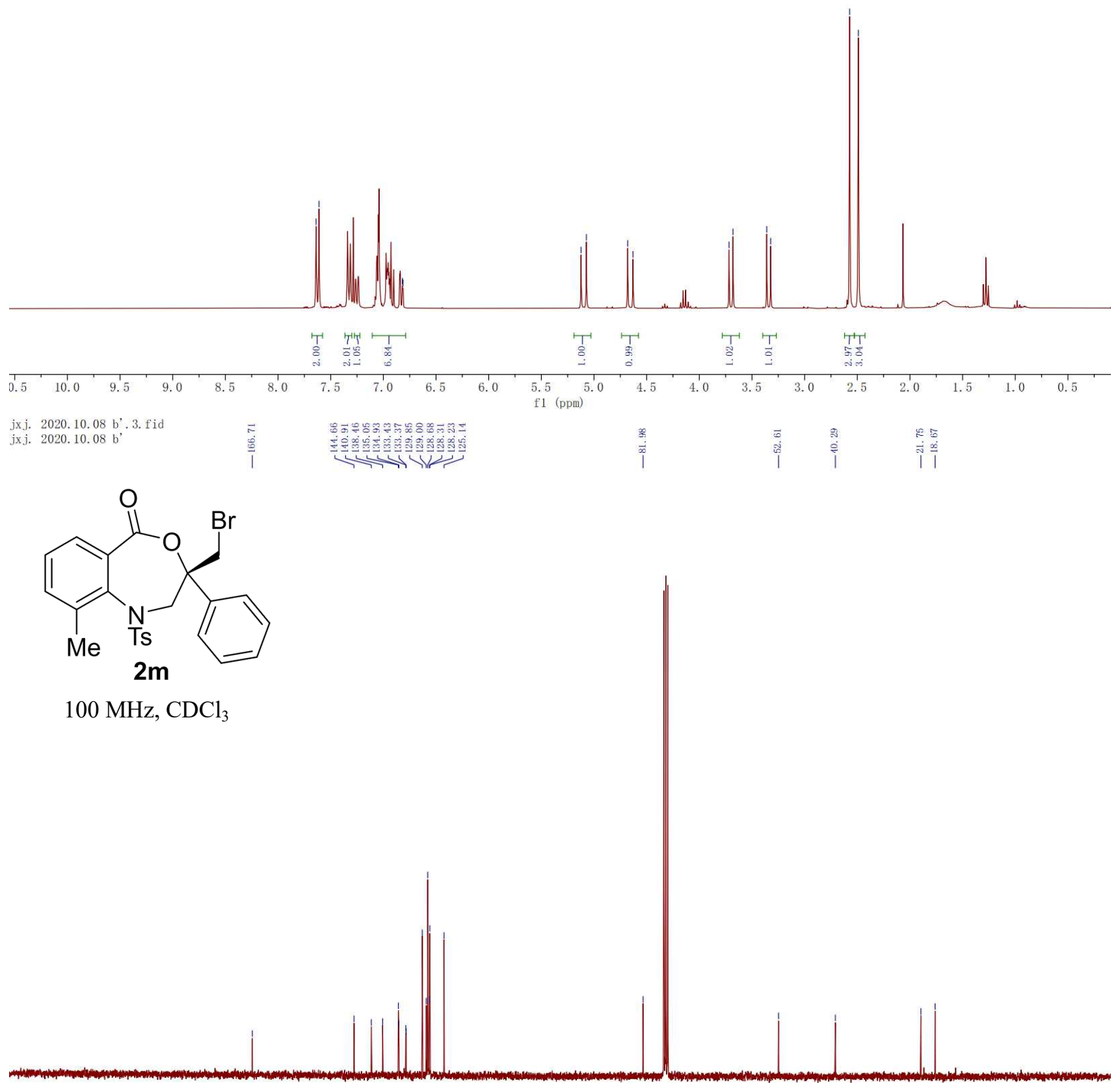




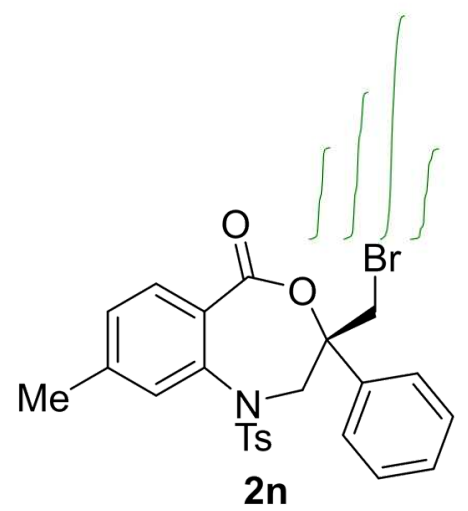

$400 \mathrm{MHz}, \mathrm{CDCl}_{3}$

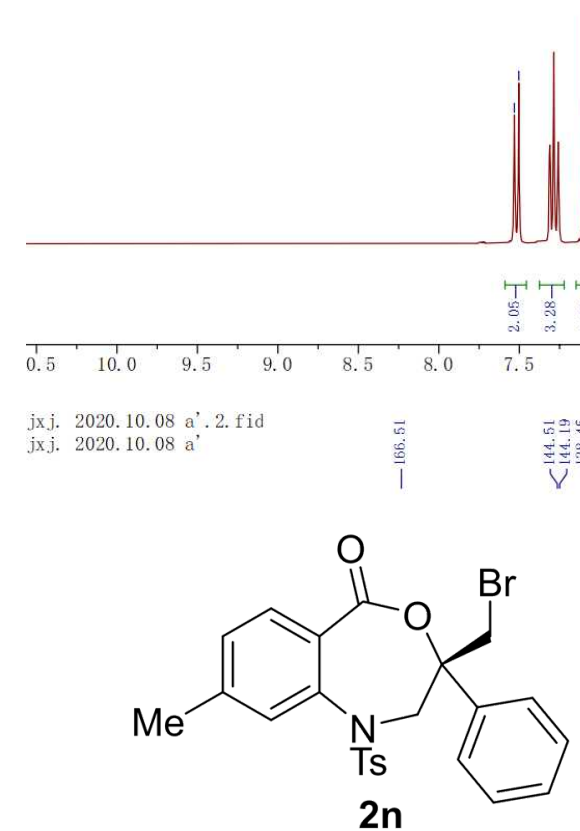

$100 \mathrm{MHz}, \mathrm{CDCl}_{3}$

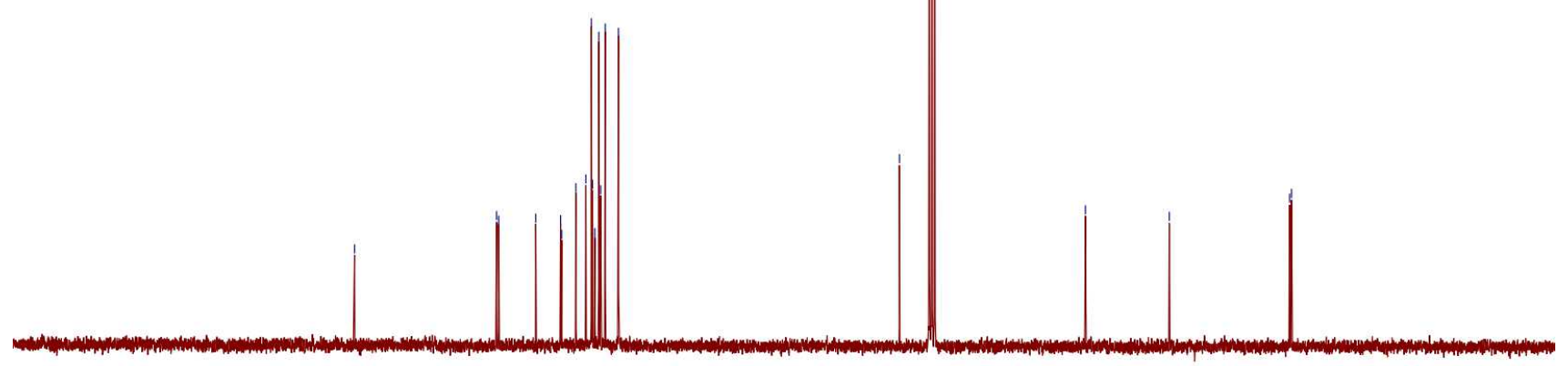


<smiles>Cc1ccc2c(c1)C(=O)O[C@@](CBr)(c1ccccc1)CN2[13C](=O)[O-]</smiles>

$400 \mathrm{MHz}, \mathrm{CDCl}_{3}$

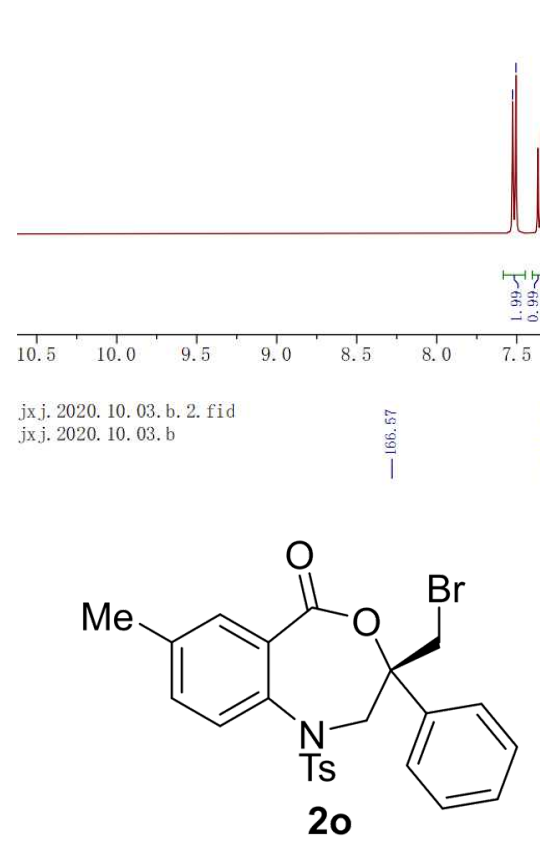

$100 \mathrm{MHz}, \mathrm{CDCl}_{3}$

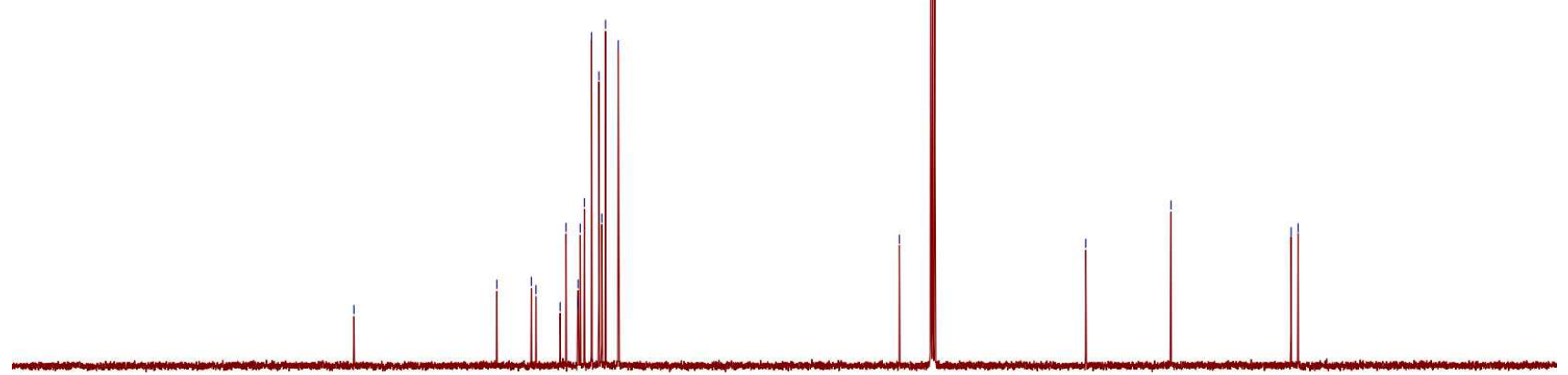




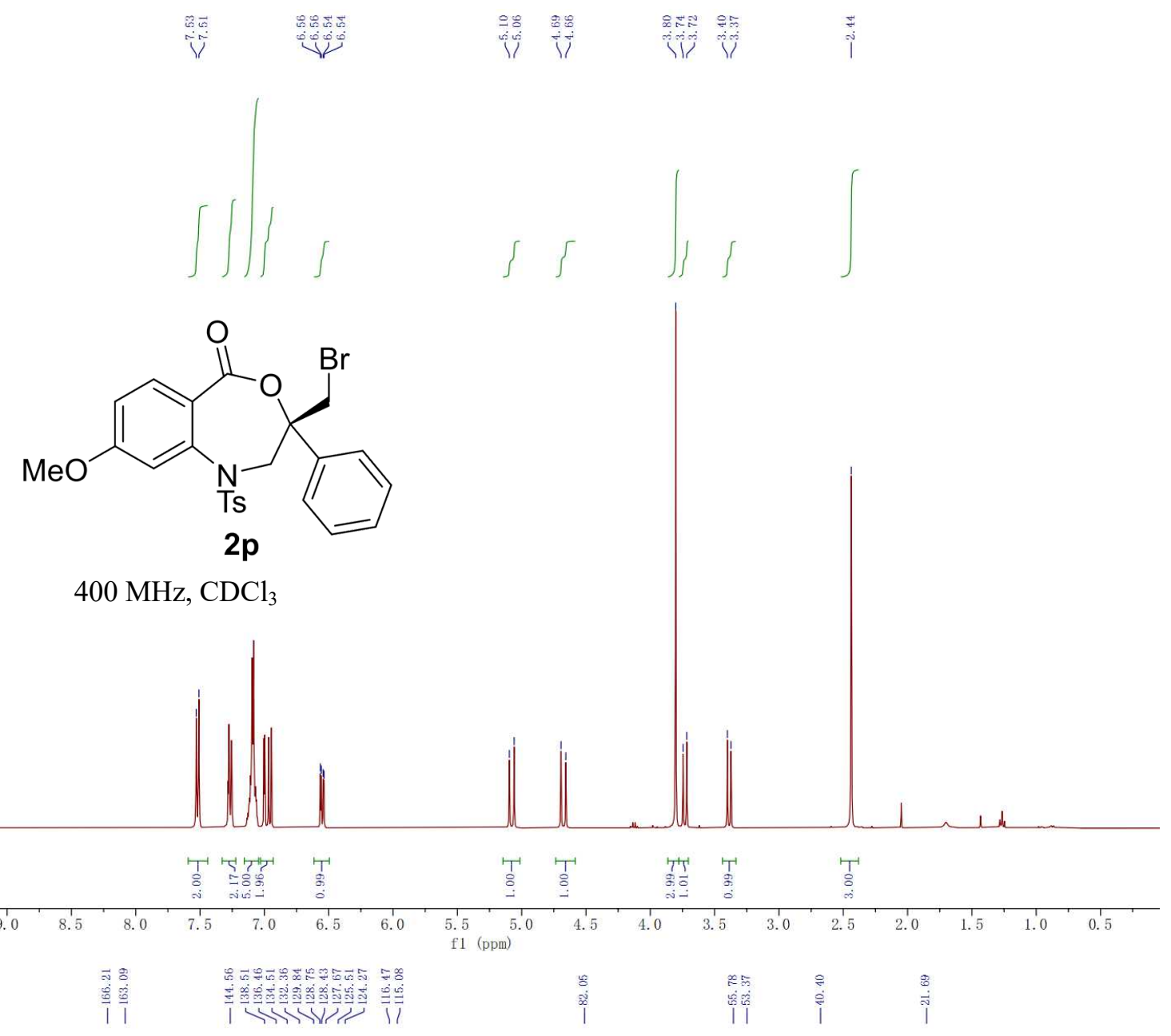<smiles>COc1ccc2c(c1)N([13CH3])C[C@@](CBr)(c1ccccc1)OC2=O</smiles>

$100 \mathrm{MHz}, \mathrm{CDCl}_{3}$

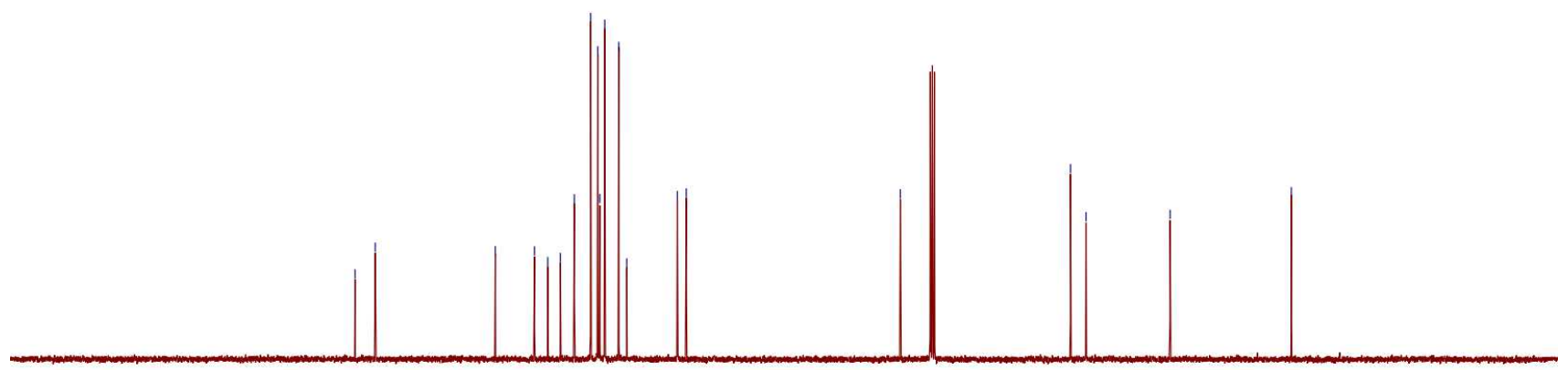

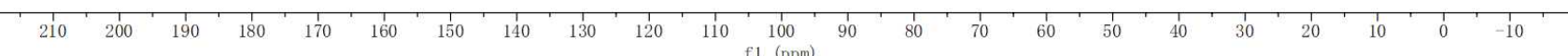


<smiles>[R16]N1C[C@@](CBr)(c2ccccc2)OC(=O)c2ccccc21</smiles>

$400 \mathrm{MHz}, \mathrm{CDCl}_{3}$

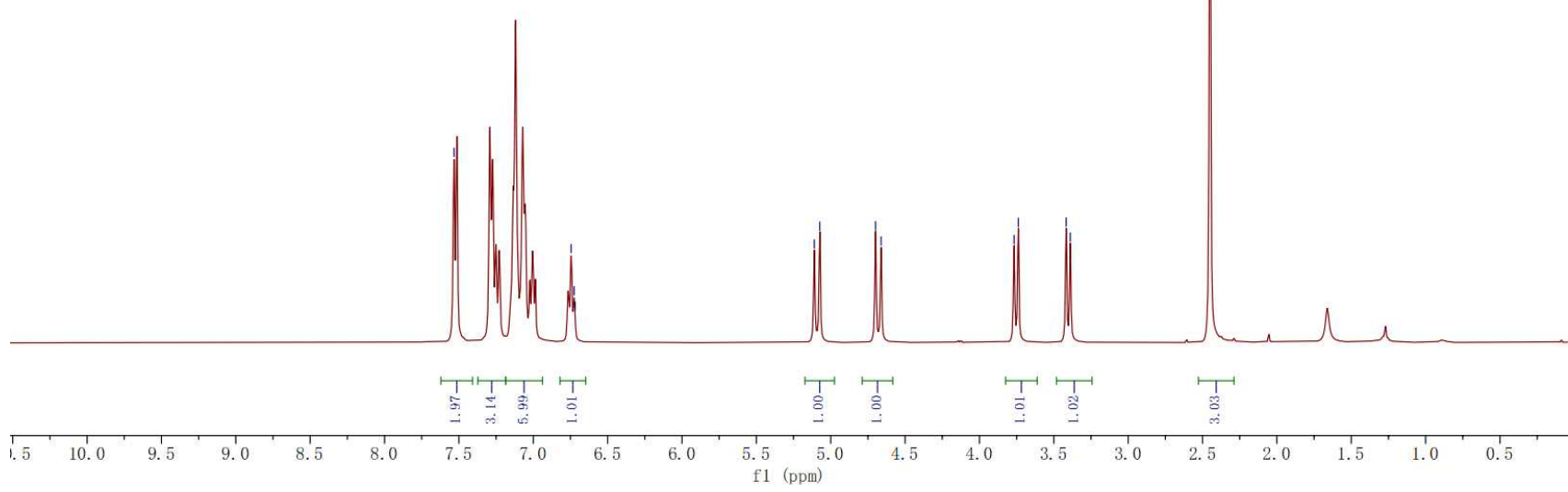

jx j. 2020. 10.28.a. 2. fid

jx j. 2020. 10. 28 . a

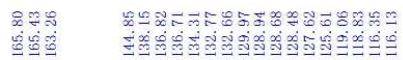

V)<smiles>CN1C[C@](CBr)(c2ccccc2)OC(=O)c2ccc(F)cc21</smiles>

$2 q$

$100 \mathrm{MHz}, \mathrm{CDCl}_{3}$

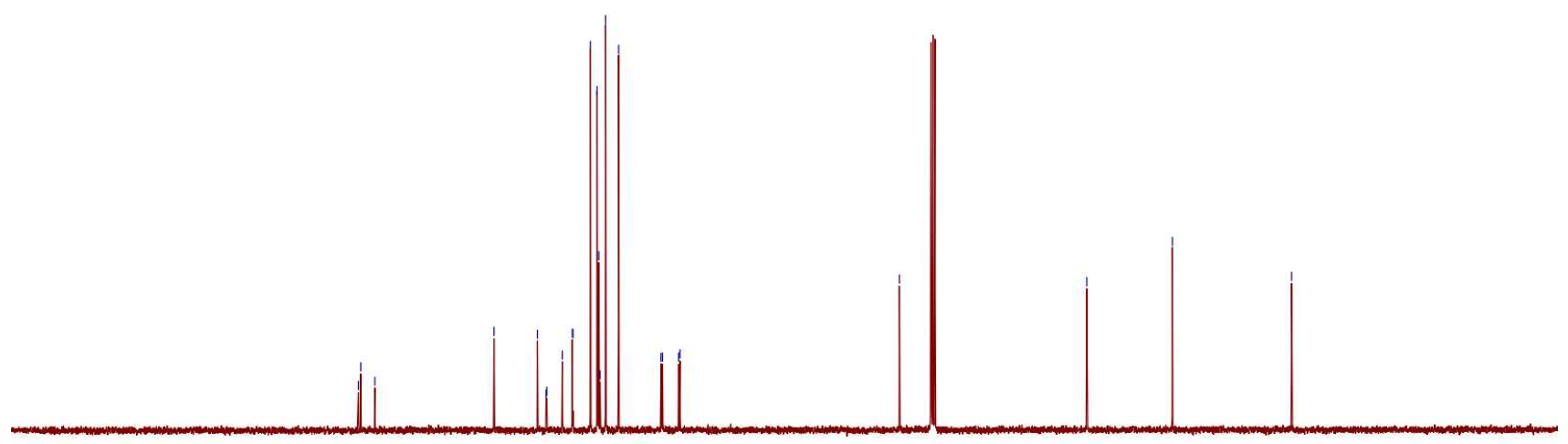

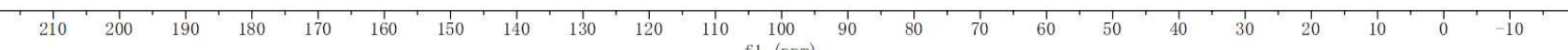




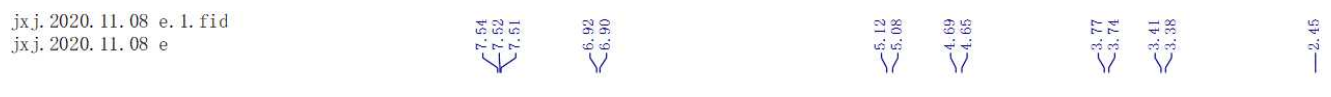

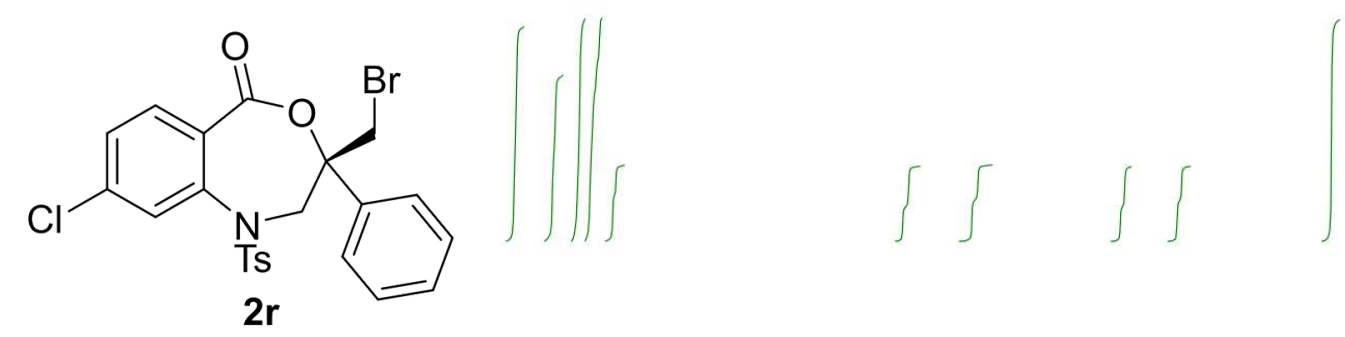

$400 \mathrm{MHz}, \mathrm{CDCl}_{3}$
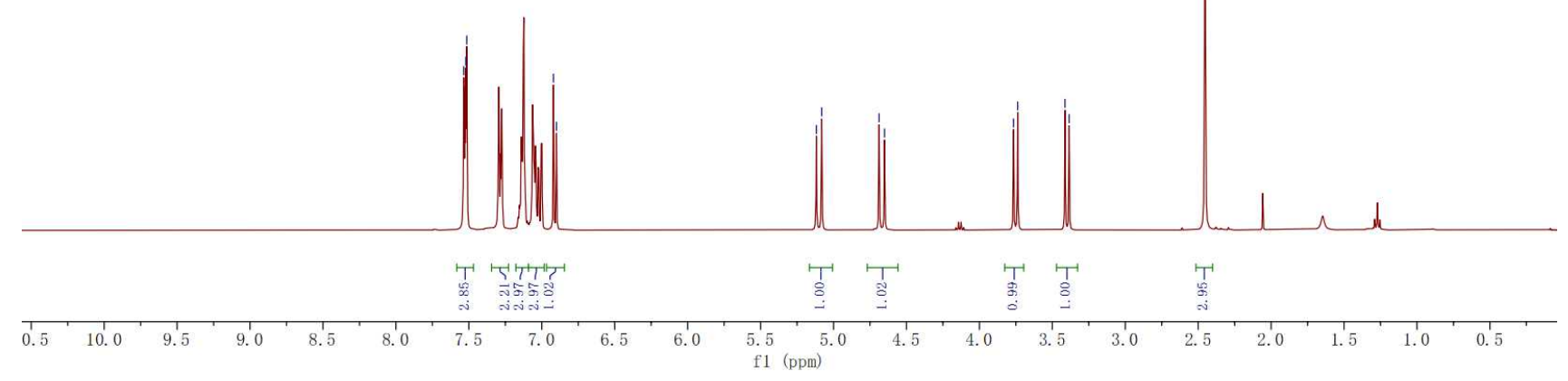

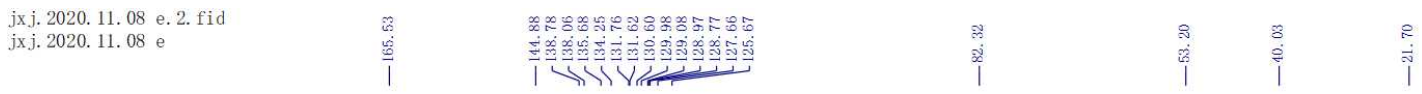<smiles>[Z17]N1C[C@@](CBr)(c2ccccc2)OC(=O)c2ccc(Cl)cc21</smiles>

$100 \mathrm{MHz}, \mathrm{CDCl}_{3}$

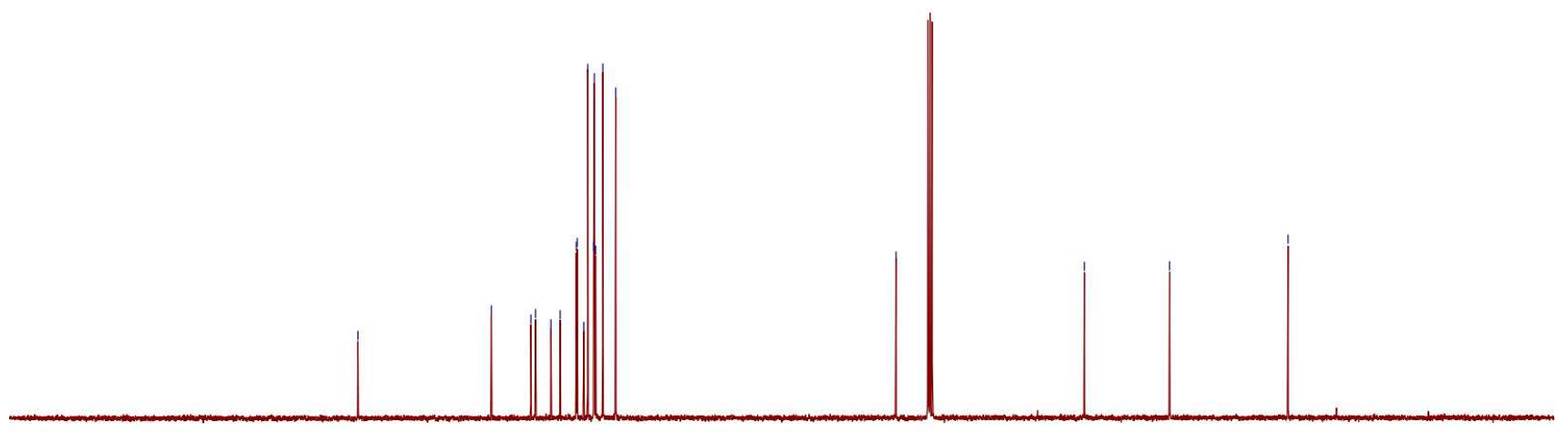

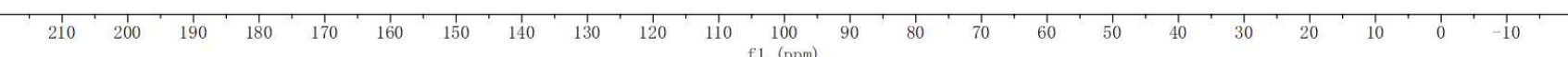


$j \mathrm{jxj} .2020 .11 .08$ b. 1. fid
$\mathrm{jx}$. 2020.11.08 b<smiles>O=C1O[C@](CBr)(c2ccccc2)CN(S)c2cc(Br)ccc21</smiles>

$400 \mathrm{MHz}, \mathrm{CDCl}_{3}$

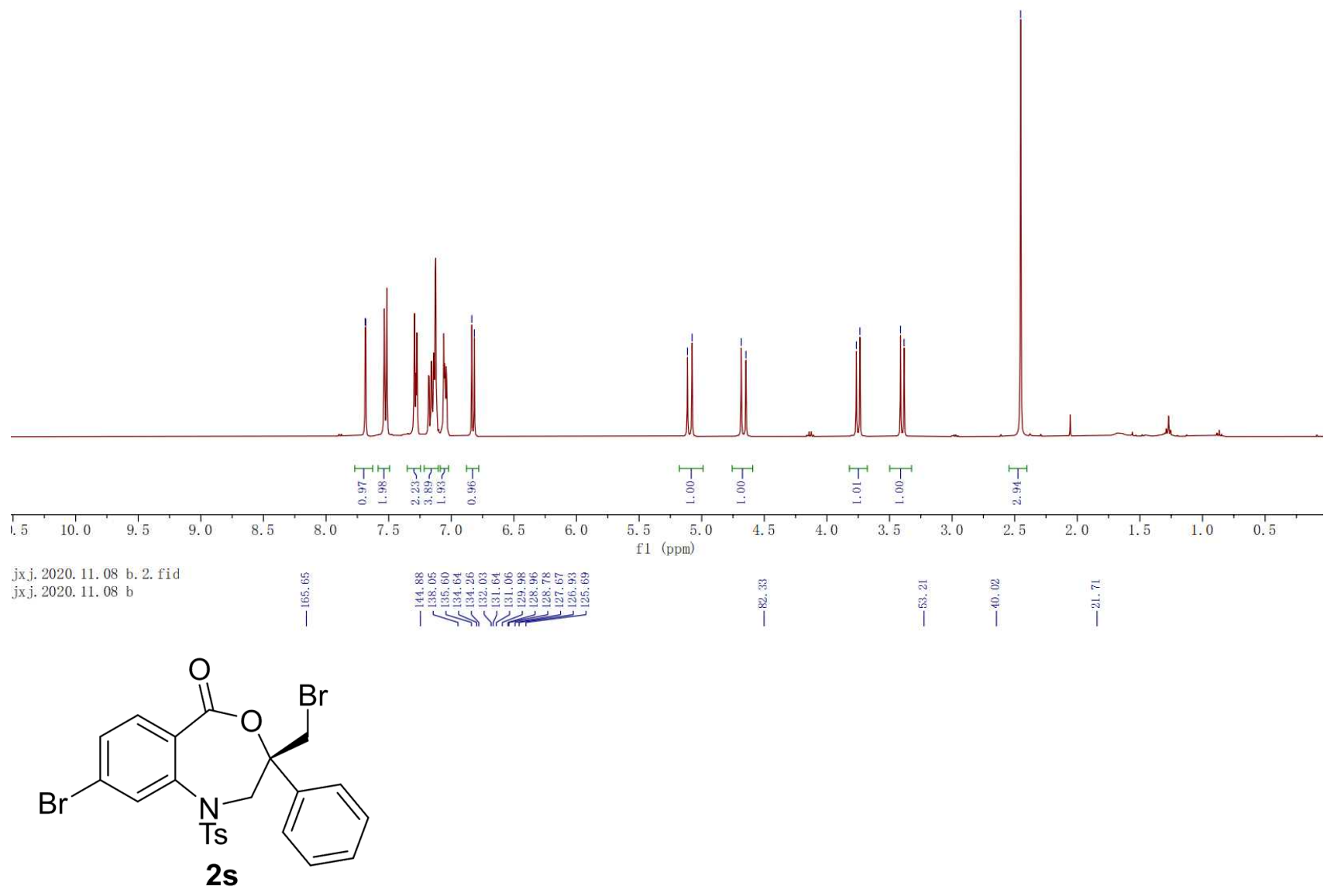

$100 \mathrm{MHz}, \mathrm{CDCl}_{3}$

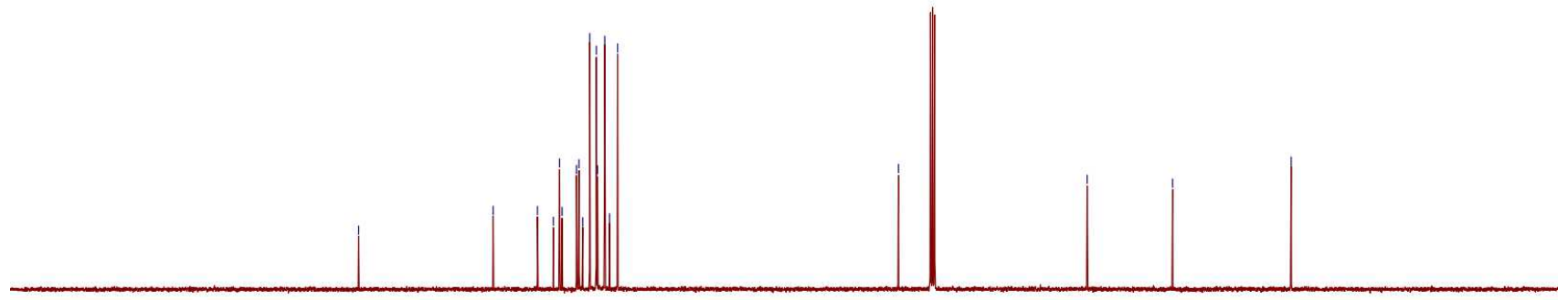

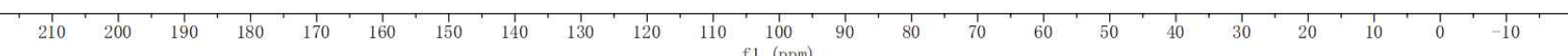


<smiles>O=C1O[C@@](CCl)(c2ccccc2)CN([As])c2ccccc21</smiles>

$400 \mathrm{MHz}, \mathrm{CDCl}_{3}$

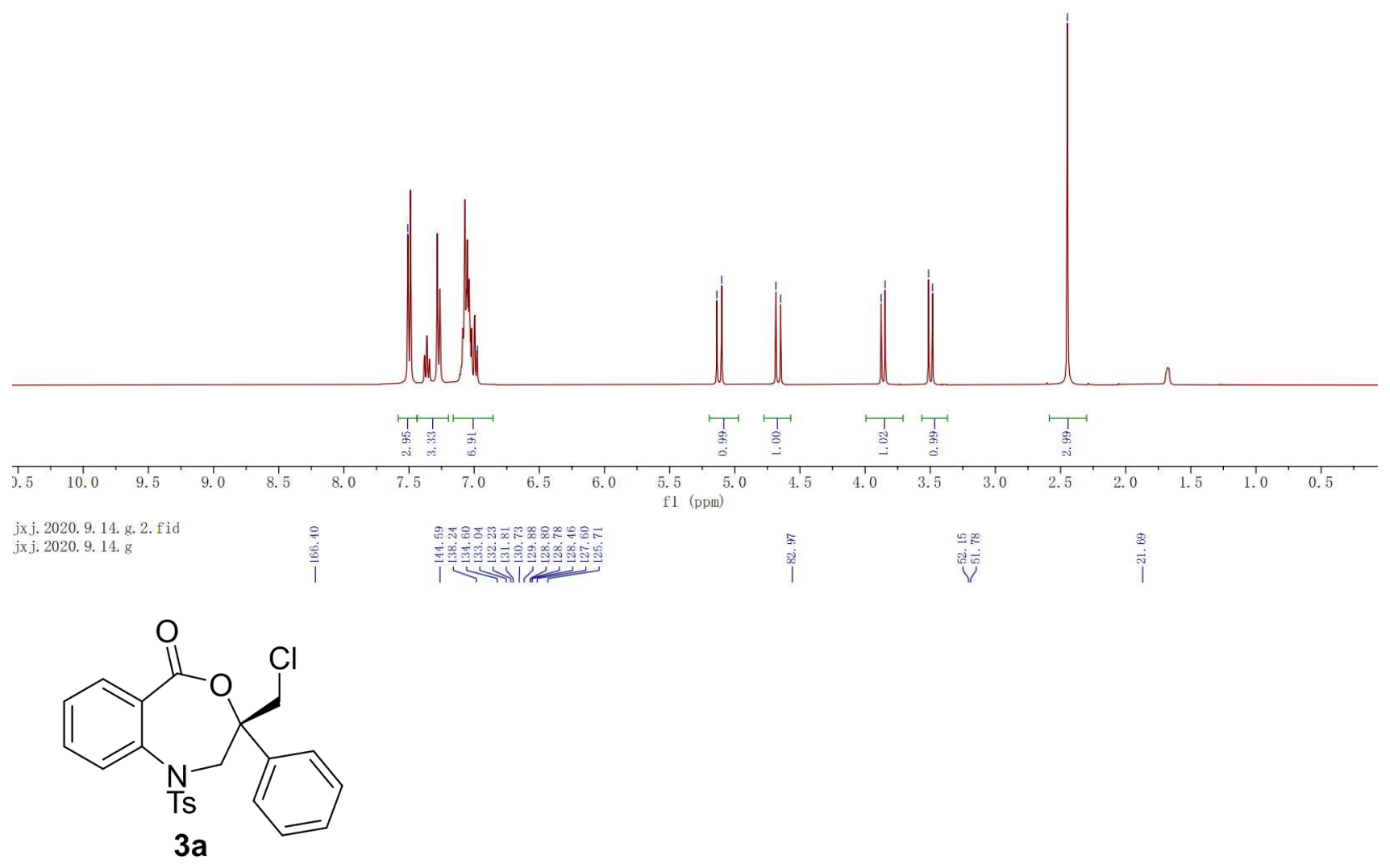

$100 \mathrm{MHz}, \mathrm{CDCl}_{3}$

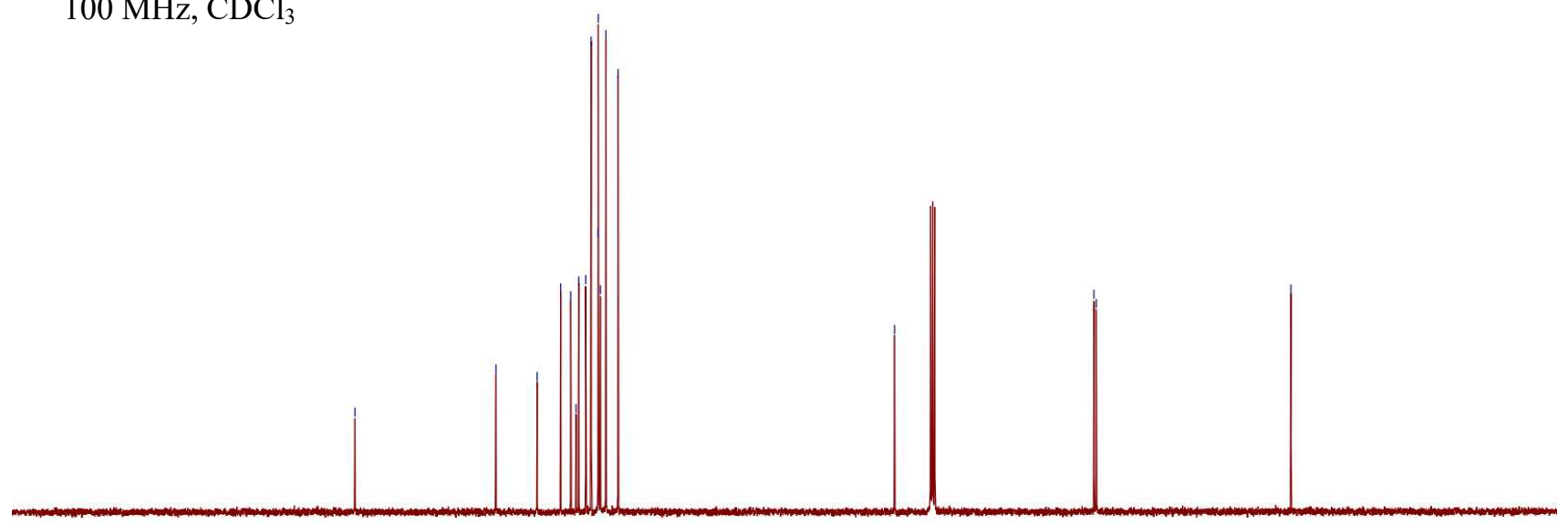

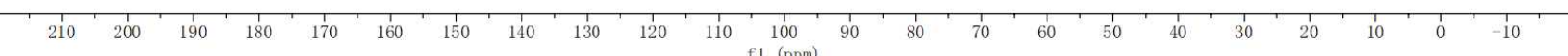


JXJ. 2020. 730. B-111. 1. fid

JXJ. 2020. 730. B-111<smiles></smiles>

$400 \mathrm{MHz}, \mathrm{CDCl}_{3}$ ili

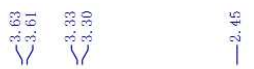

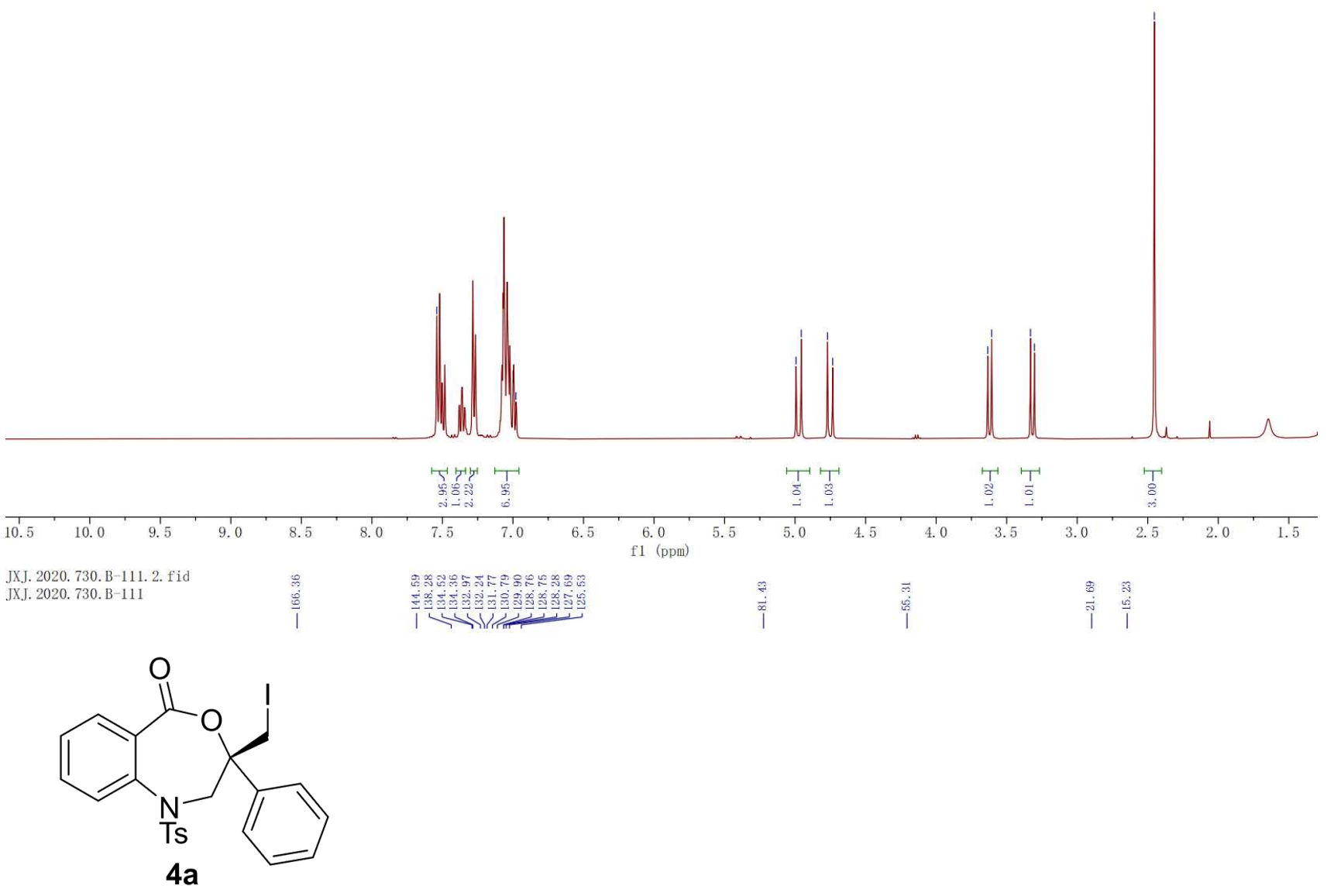

$100 \mathrm{MHz}, \mathrm{CDCl}_{3}$

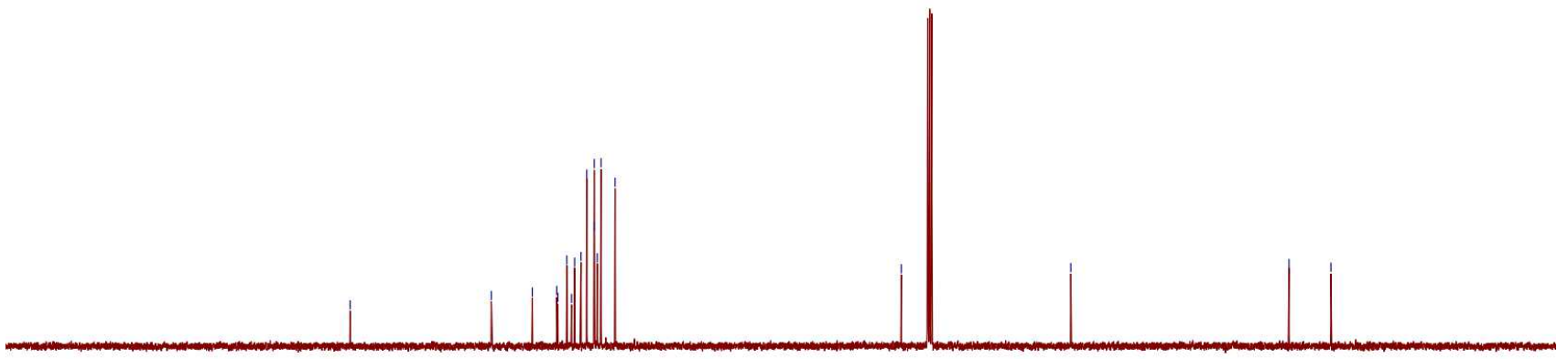

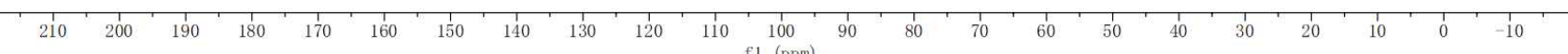


<smiles></smiles>

$6 a$

$400 \mathrm{MHz}, \mathrm{CDCl}_{3}$
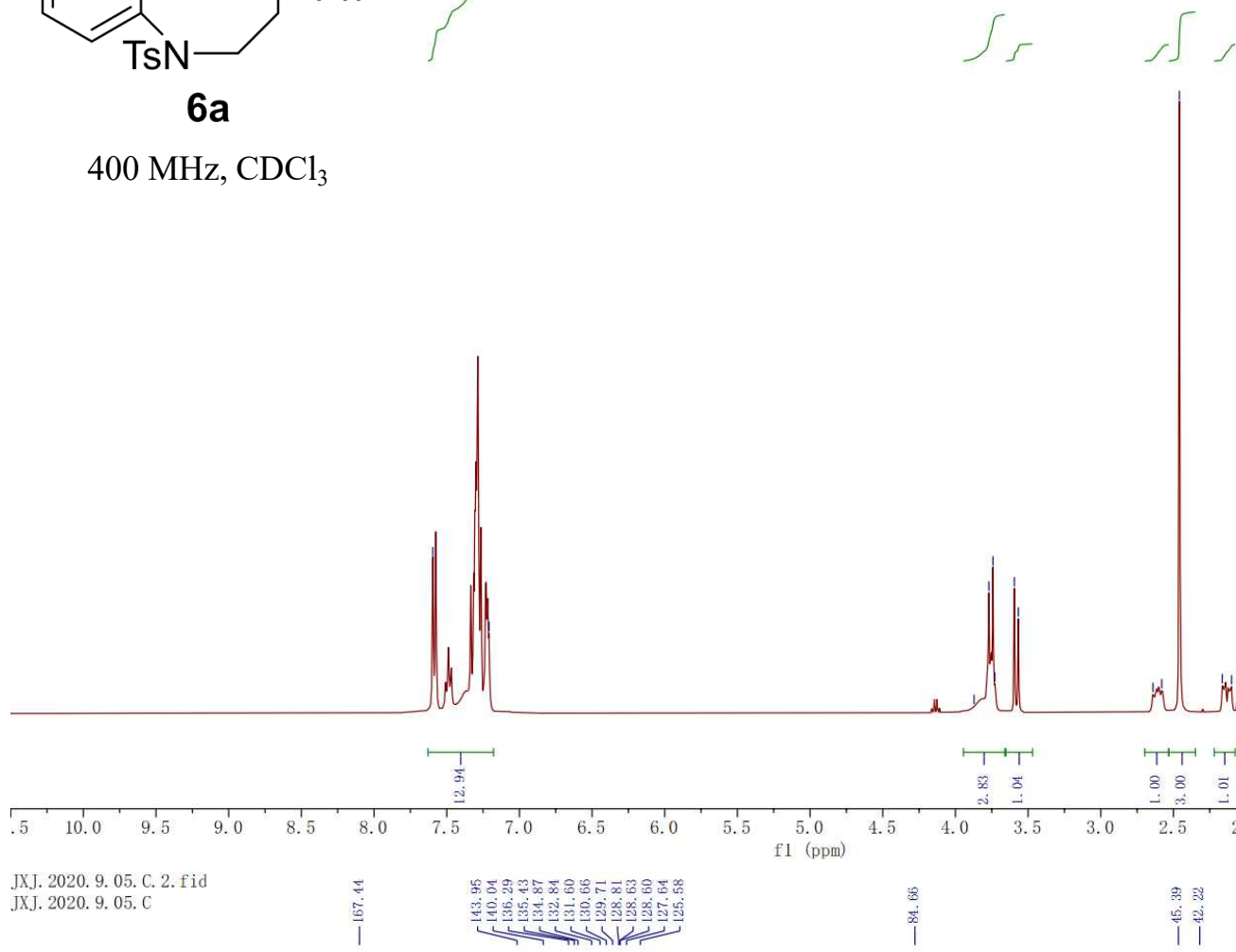<smiles>O=C1O[C@](CBr)(c2ccccc2)CCN2[13CH]=C[13CH]12</smiles>

$6 a$

$100 \mathrm{MHz}, \mathrm{CDCl}_{3}$

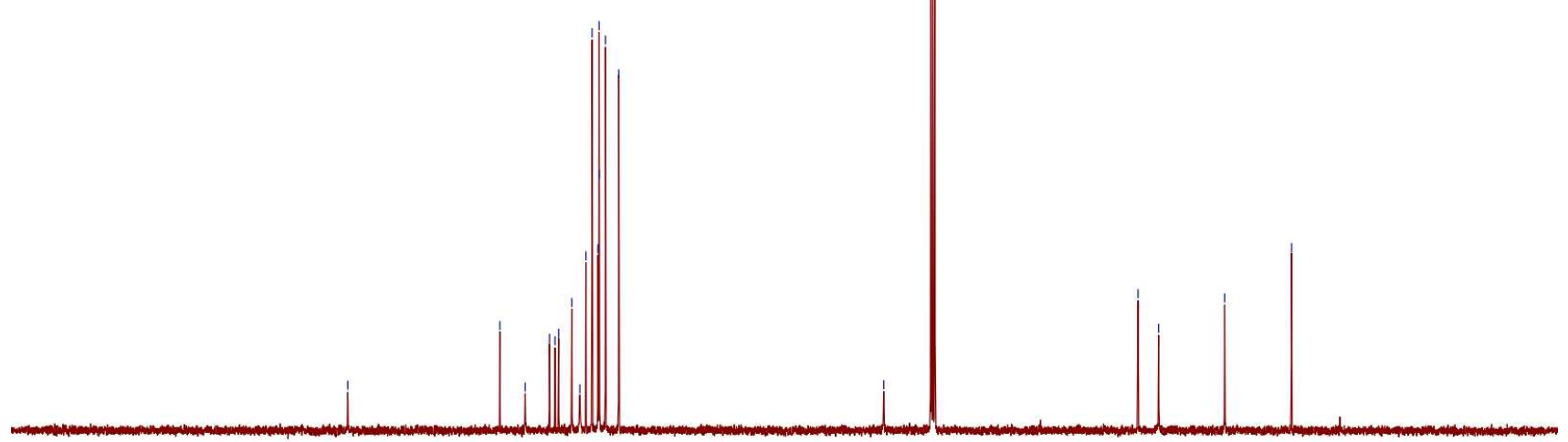

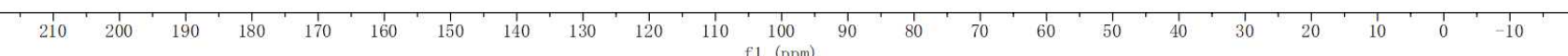


<smiles>O=C1O[C@](CBr)(c2ccccc2)COc2ccccc21</smiles>

6b

$400 \mathrm{MHz}, \mathrm{CDCl}_{3}$

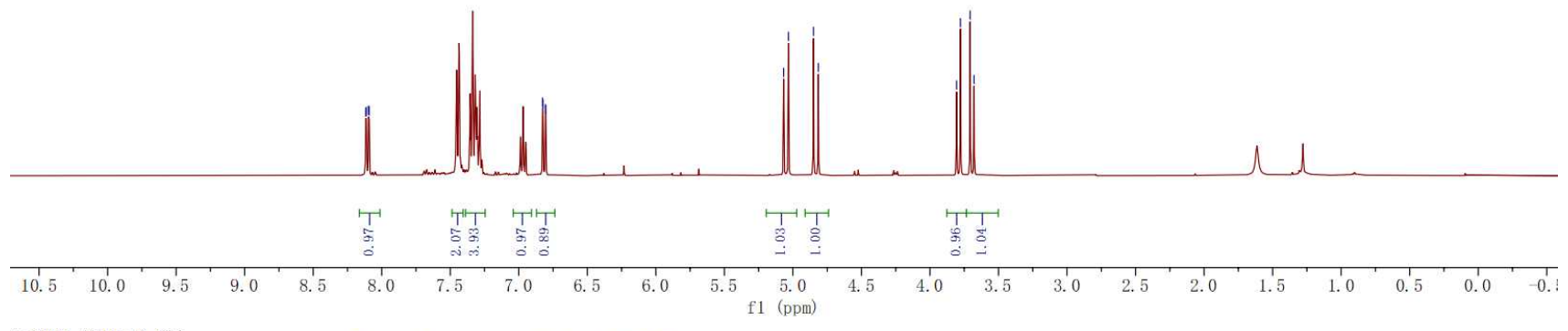

jx j2021. 0719a. 2. fid

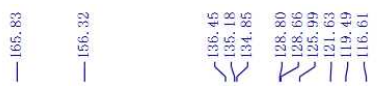

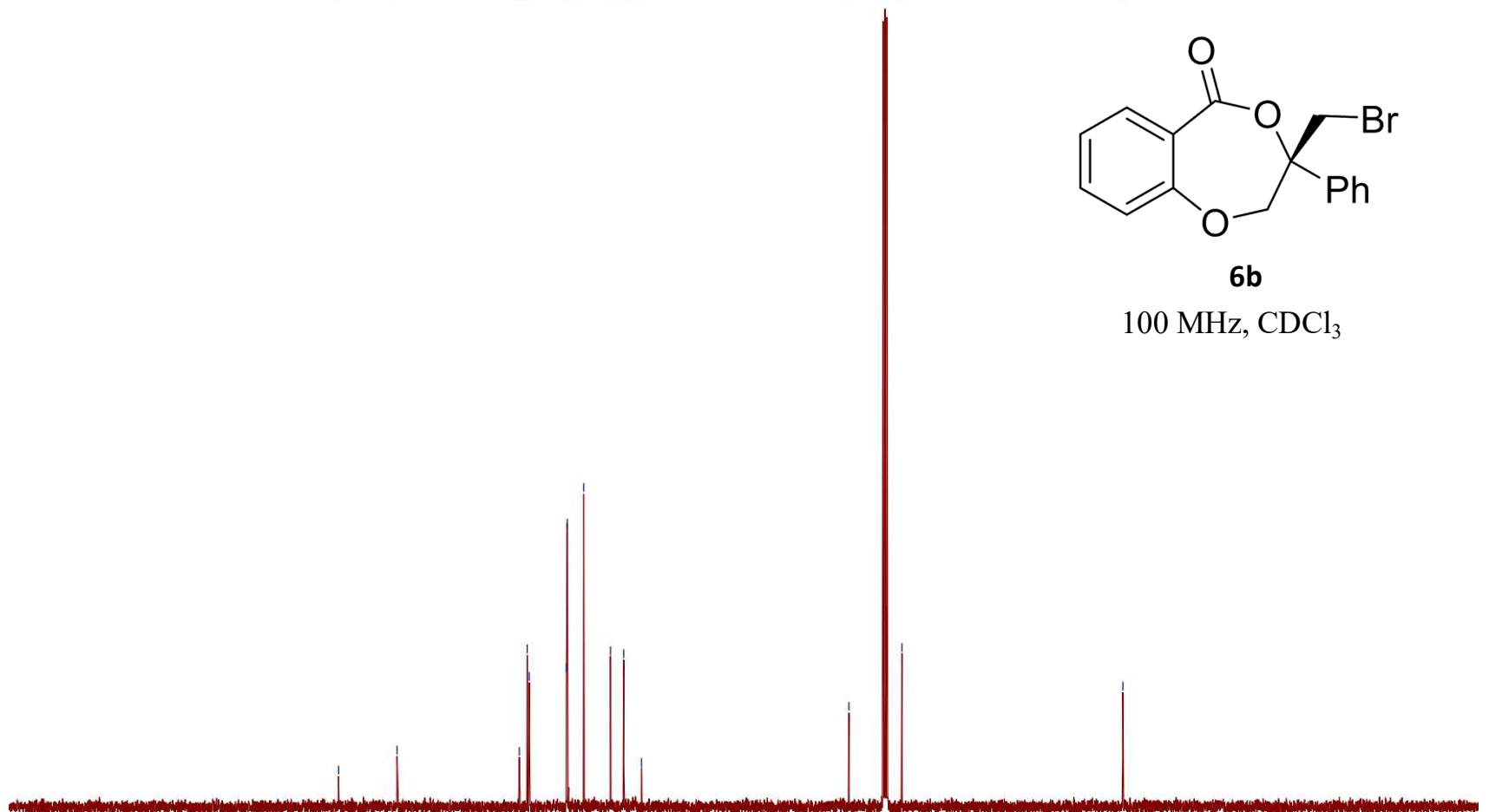


<smiles>O=C1O[C@H](c2ccccc2)C(Cl)CN(S)c2ccccc21</smiles>

8

$400 \mathrm{MHz}, \mathrm{CDCl}_{3}$
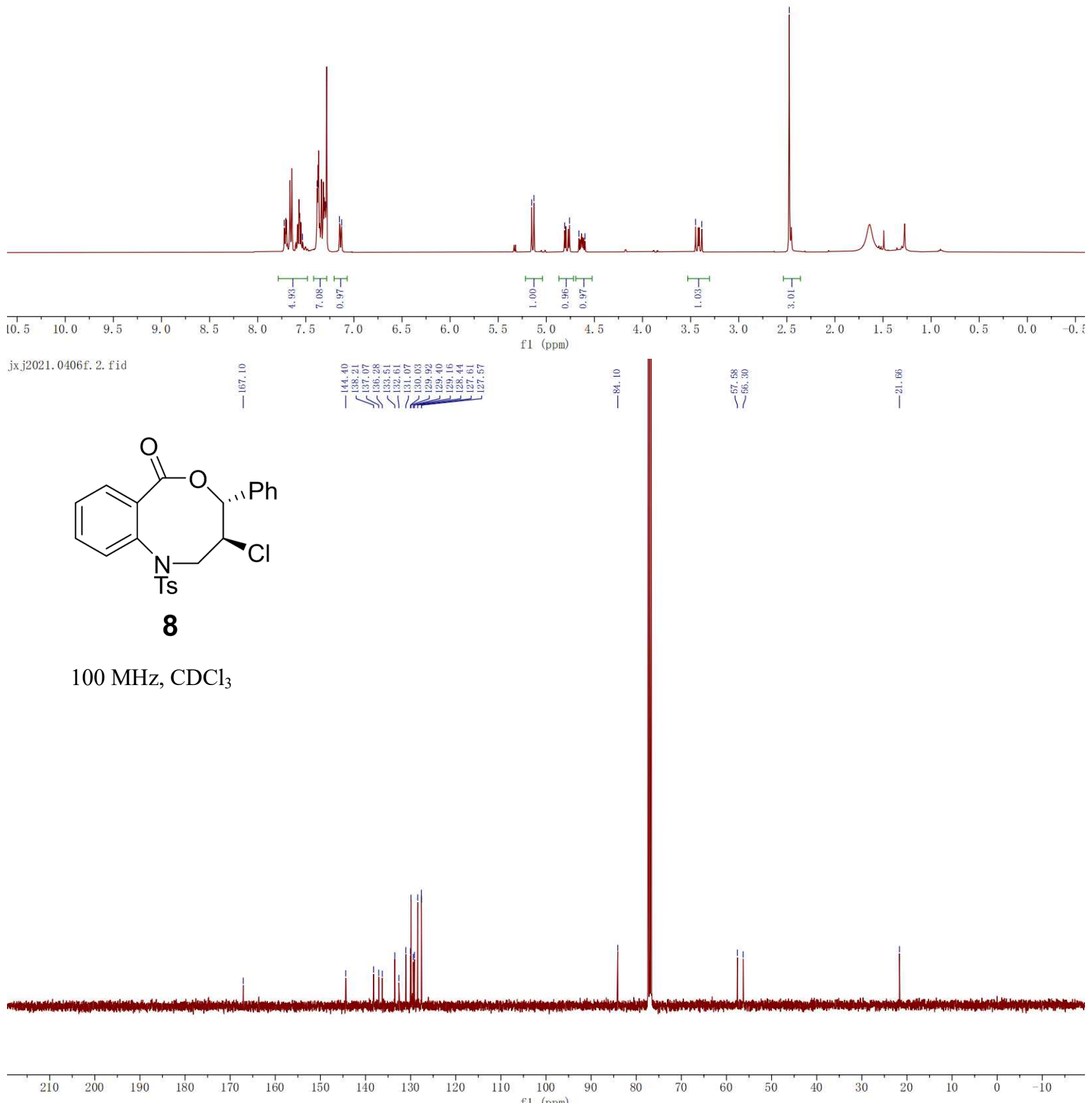


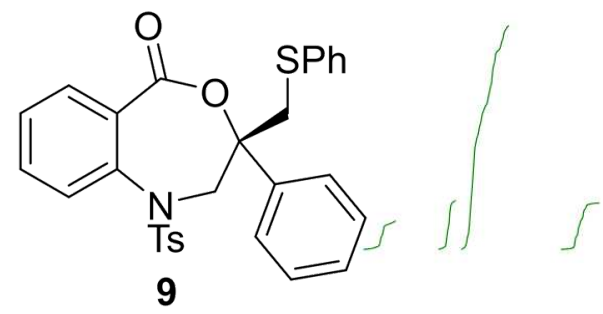

$400 \mathrm{MHz}, \mathrm{CDCl}_{3}$

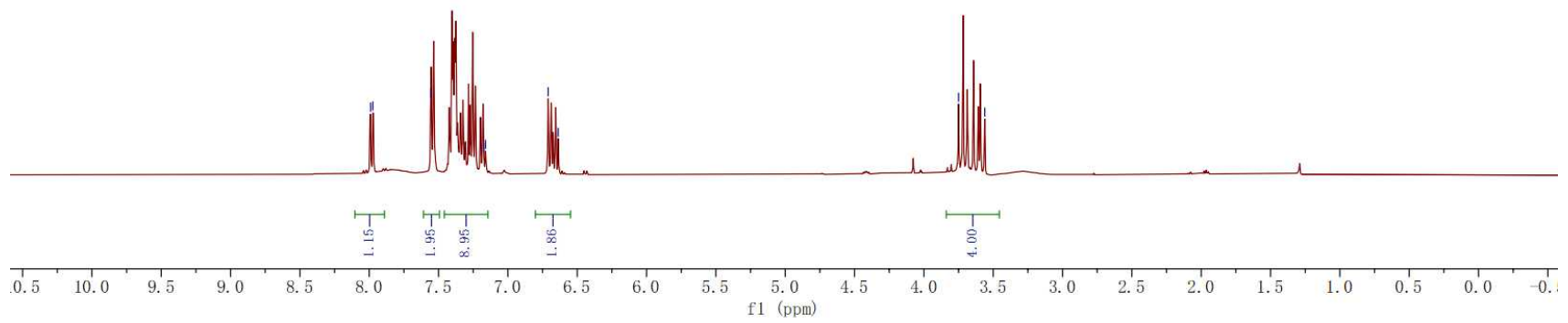

JXJ2021. 0424A. 2. fid

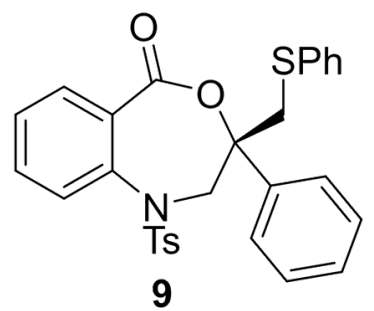

$100 \mathrm{MHz}, \mathrm{CDCl}_{3}$
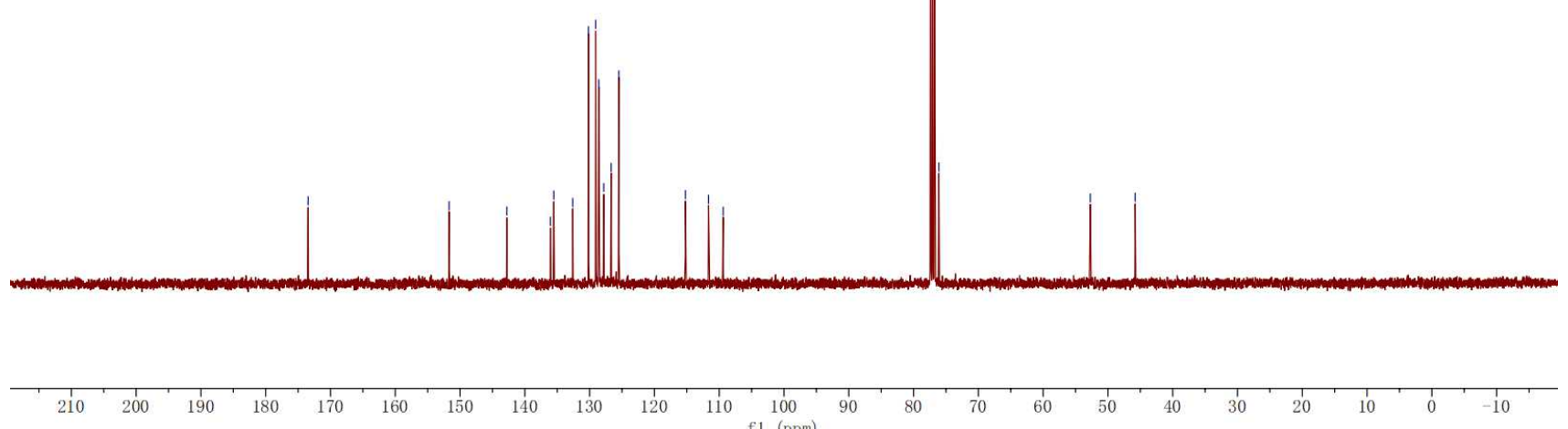